\title{
Andreas Ickes
}

\section{Parteiprogramme \\ Sprachliche Gestalt und Textgebrauch}

Die Macht der Sprache
Sprache Die Macht der S
der Sprache Die Macht
Macht der Sprache Die N
Die Macht der Sprache
Sprache Die Macht der S
der Sprache Die Macht
Macht der Sprache Die N

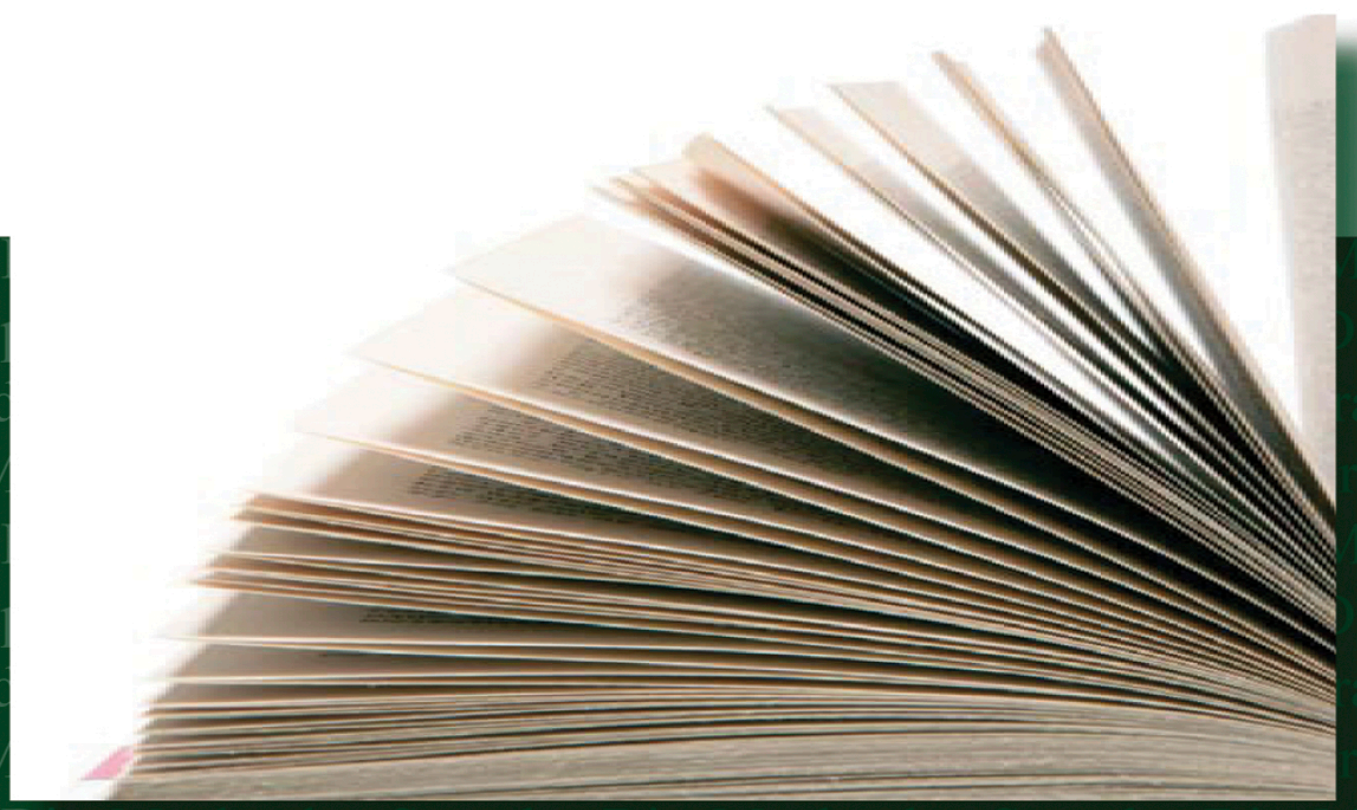

Die Macht der Sprache

Sprache Die Macht der Sprache Die Macht der Sprache Die Macht der Sprache Die M der Sprache Die Macht der Sprache Die Macht der Sprache Die Macht der Sprache Macht der Sprache Die Macht der Sprache Die Macht der Sprache Die Macht der Spr Die Macht der Sprache Die Macht der Sprache Die Macht der Sprache Die Macht prache Die Macht der Sprache Die Macht der Sprache Die Macht der Sprache Die M ler Sprache Die Macht der Sprache Die Macht der Sprache Die Macht der Sprache Macht der Sprache Die Macht der Sprache Die Macht der Sprache Die Macht der Spr Die Macht der Sprache Die Macht der Sprache Die Macht der Sprache Die Macht Sprache Die Macht der Sprache Die Macht der Sprache Die Macht der Sprache Die M der Sprache Die Macht der Sprache Die Macht der Sprache Die Macht der Sprache Macht der Sprache Die Macht der Sprache Die Macht der Sprache Die Macht der Spra Die Macht der Sprache Die Macht der Sprache Die Macht der Sprache Die Macht Sprache Die Macht der Sprache Die Macht der Sprache Die Macht der Sprache Die M Ler Sprache Die Macht der Sprache Die Macht der Sprache Die Büchner 
Andreas Ickes

Parteiprogramme Sprachliche Gestalt und Textgebrauch 

Andreas Ickes

Parteiprogramme

Sprachliche Gestalt und Textgebrauch 
www.buechner-verlag.de

\section{(c) $(1) \Theta$}

Dieses Werk erscheint unter der Creative-Commons-Lizenz 4.0 (CC-BY-NC). Diese Lizenz erlaubt unter dem Vorbehalt der Namensnennung des Urhebers die Bearbeitung, Vervielfältigung und Verbreitung des Materials in jedem Format oder Medium, jedoch nur für nicht kommerzielle Zwecke. Die Bedingungen der Creative-Commons-Lizenz gelten nur für Originalmaterial. Die Wiederverwendung von Material aus anderen Quellen (gekennzeichnet mit Quellenangabe) wie z. B. Schaubilder, Abbildungen, Fotos und Textauszüge erfordert ggf. weitere Nutzungsgenehmigungen durch den jeweiligen Rechteinhaber.

ISBN (Print): 978-3-941310-03-2

ISBN (PDF): 978-3-96317-708-8

DOI: $10.14631 / 978-3-96317-708-8$

Erschienen 2008 im Büchner-Verlag

Bibliografische Informationen der Deutschen Nationalbibliothek Die Deutsche Nationalbibliothek verzeichnet diese Publikation in der Deutschen Nationalbibliografie, detaillierte bibliografische Angaben sind im Internet über http://dnb.de abrufbar. 


\section{INHALT}

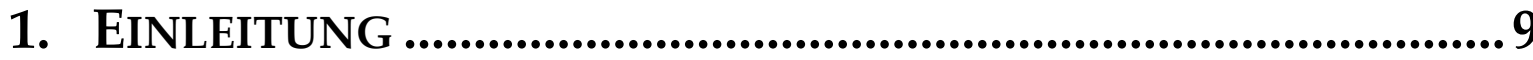

1.1 Die Fragestellung.....................................................................................9

1.2 Parteiprogramme: Spielarten und historische Entwicklung ....11

1.2.1 Das Parteiprogramm als Textsorte ......................................................12

1.2.1.1 Das Parteiprogramm als Sprachspiel................................................ 13

1.2.1.2 Wahl-/Aktionsprogramme ............................................................ 16

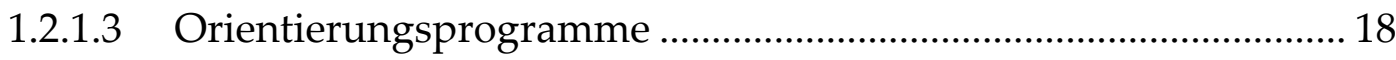

1.2.1.4 Grundsatzprogramme ..................................................................... 18

1.2.1.4.1 Historische Hintergründe des Godesberger Programms ............. 20

1.3 Themenspektrum und Ausblick .........................................................26

1.3.1 Methoden der Untersuchung und Aufbau der Arbeit.................26

2. EXEMPLARISCHE ANALYSEN DER PARTEIPROGRAMME........29

2.1 Pragmatische Aspekte der Textorganisation...................................29

2.1.1 Zum Verhältnis von Sprache und Politik ..........................................30

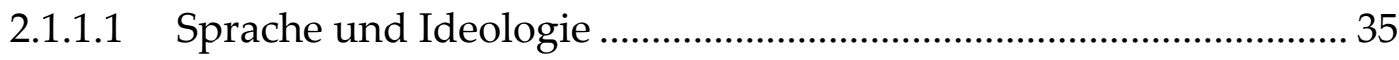

2.1.1.2 Sprache und gesellschaftliche Wirklichkeit ....................................... 37

2.1.1.2.1 Parteiprogramme - Wirklichkeitsabbildung und Fachsprachlichkeit................................................................. 38

2.1.2 Funktionen von Sprache ......................................................................40

2.1.2.1 Funktionen der politischen Sprachverwendung .............................. 41

2.1.2.1.1 Das regulative Sprachspiel/

Die regulative Sprachfunktion....................................................... 44

2.1.2.1.2 Das instrumentale/begehrende Sprachspiel/

Die poskative Sprachfunktion 44

2.1.2.1.3 Das integrative Sprachspiel/

Die integrative Sprachfunktion 45

2.1.2.1.4 Das informativ-persuasive Sprachspiel/

Die informativ-persuasive Sprachfunktion ................................. 46

2.1.2.2 Funktionen von Parteiprogrammen ................................................. 48

2.1.2.2.1 Wahl-/Aktionsprogramme ....................................................... 52

2.1.2.2.2 Grundsatzprogramme .............................................................. 54

2.1.2.2.3 Wahlprogramm vs. Grundsatzprogramm ................................. 55 
2.1.3 Das Spektrum der sprachlichen Handlungsformen ...................57

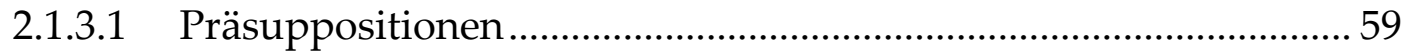

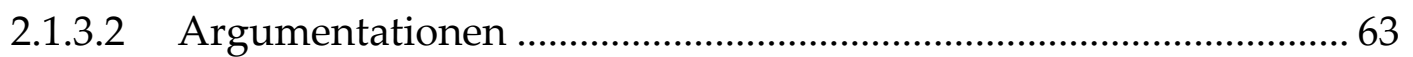

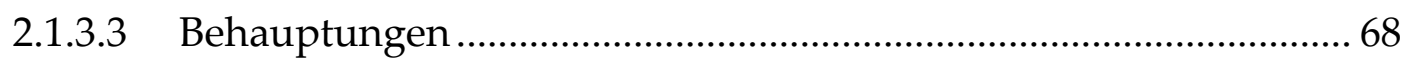

2.1.3.4 Anspruch auf Erfüllbarkeit ....................................................... 70

2.1.3.5 Versprechen/Zielbestrebungen................................................... 71

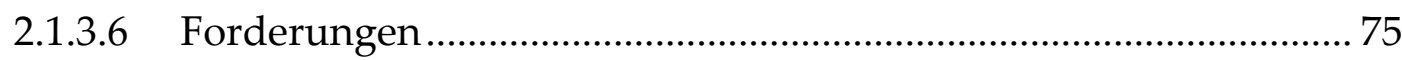

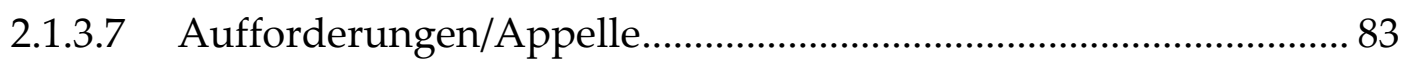

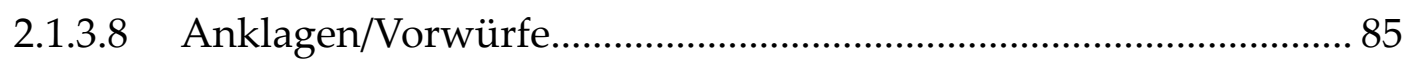

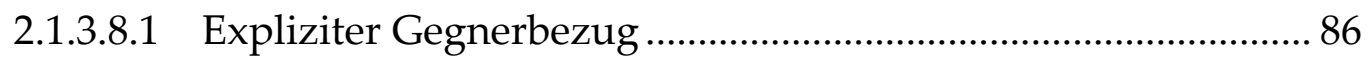

2.1.4 Sonderformen politischer Sprachverwendung ..............................88

2.1.4.1 Bewusste Nicht-Erwähnungen ................................................... 91

2.1.4.2 Kompromiss-Formulierungen .................................................... 93

2.1.4.3 Kalkulierte Ambivalenz .................................................................. 96

2.1.4.4 Das Godesberger Programm und der Grundgesetztext -

Gemeinsamkeiten und Unterschiede .......................................... 101

2.1.4.4.1 Die Präambeln im Godesberger Programm und im Grundgesetz ........................................................... 111

2.1.4.5 Das Parteiprogramm als Bekenntnisschrift ............................... 117

2.1.5 Zur Rezeption von Parteiprogrammen ..........................................119

2.1.5.1 Expliziter Adressatenbezug.....................................................121

2.1.5.1.1 Das Personalpronomen wir ..................................................... 126

2.1.5.2 Impliziter Adressatenbezug ..................................................... 133

2.1.6 Selbstverständlichkeiten ............................................................134

2.1.7 Phrasenhaftigkeit in den Parteiprogrammen ...........................135

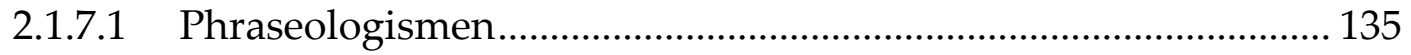

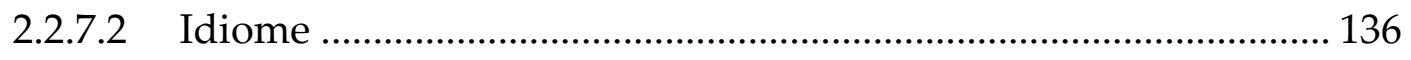

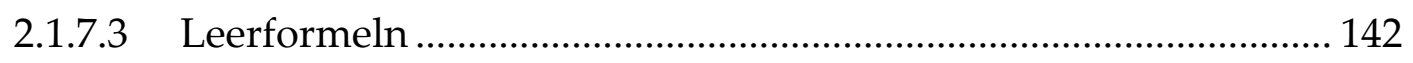

2.1.8 Aspekte der stilistischen Beschreibung........................................154

2.2 Aspekte der sprachlichen Gestalt ...................................................158

2.2.1 Lexik/Semantik: Wortschatz-Charakteristik ..............................158

2.2.1.1 Grundlegende Annahmen zur Wortschatzbetrachtung .............. 159

2.2.1.2 Systemstellen............................................................................... 163

2.2.1.2.1 Wortschatzsektoren, Wortfamilien und Wortfelder ............... 166

2.2.1.3 Erscheinungsformen von Wortbildungsprozessen ..................... 179 


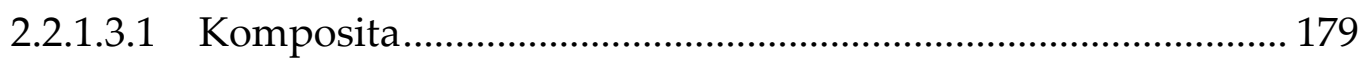

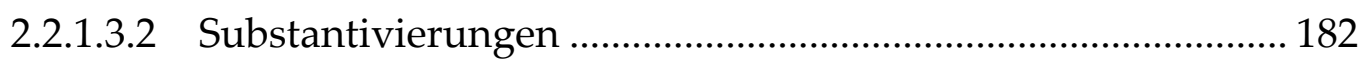

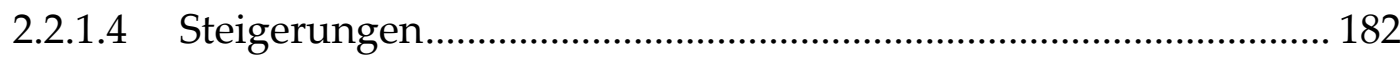

2.2.1.5 Kandidaten für Archaismen/Neologismen.................................. 183

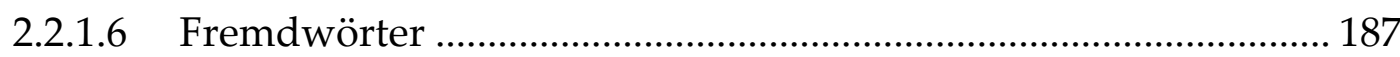

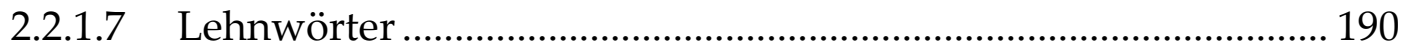

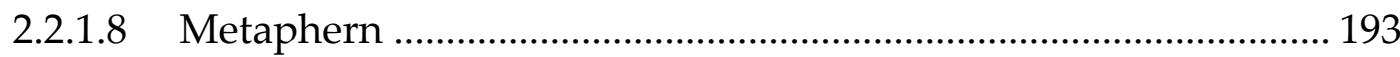

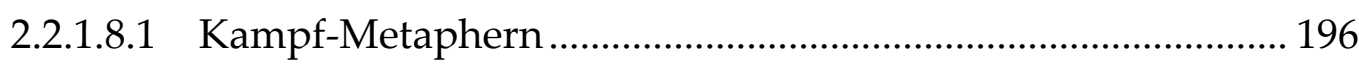

2.2.1.8.2 Nautische Metaphern/Schiffs-Metaphern.............................. 200

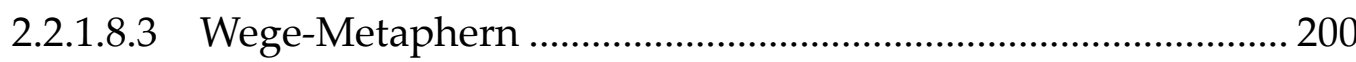

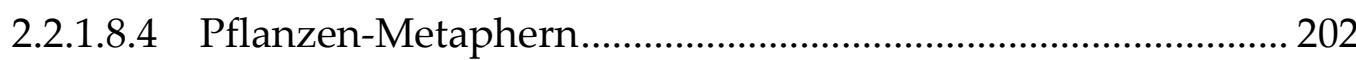

2.2.1.8.5 Bau- oder Gebäude-Metaphern ............................................... 203

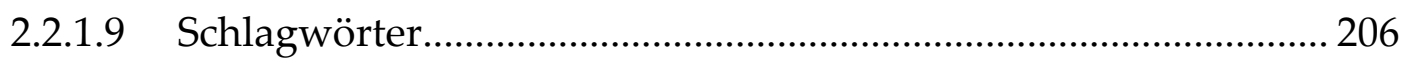

2.2.1.9.1 Fahnen- und Stigmawörter ................................................... 208

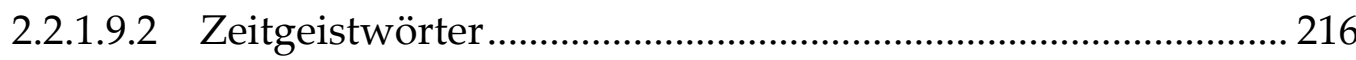

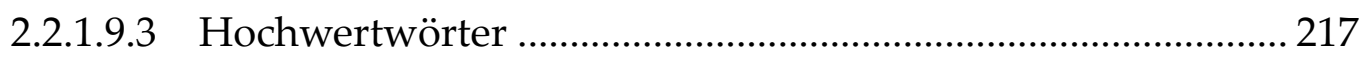

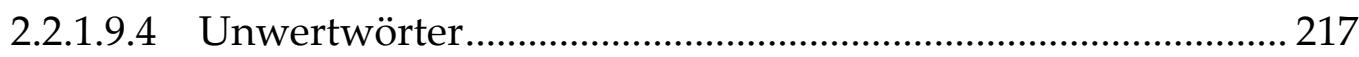

2.2.1.9.5 Programmwörter .................................................................... 217

2.2.1.9.6 Stich- oder Themawörter ..................................................... 218

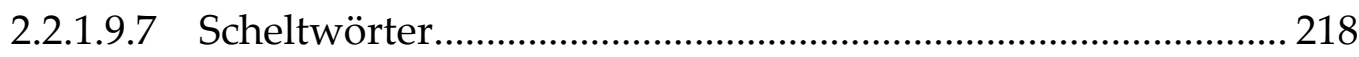

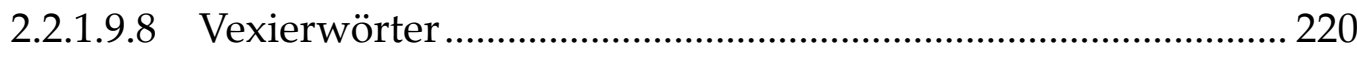

2.2.1.10 Erzeugung von Sichtweisen................................................. 223

2.2.1.10.1 Erzeugung positiver Sichtweisen/Euphemismen .................. 224

2.2.1.10.2 Erzeugung negativer Sichtweisen ........................................ 227

3. ERGEBNISSE UND ZUSAMMENFASSUNG ............................. 229

4. BIBLIOGRAPHIE .................................................................. 233

4.1 Primärliteratur..................................................................................233

4.2 Sekundärliteratur.......................................................................233

4.3 Quellen im Internet....................................................................243 



\section{EINLEITUNG}

Die Regierungsform der Bundesrepublik Deutschland basiert auf dem Prinzip der repräsentativen und parlamentarischen Demokratie. Kennzeichnend für eine solche parlamentarische Demokratie ist, dass die größte Macht beim Parlament liegt; ohne eine Mehrheit im Parlament können keine weiterreichenden politischen Entscheidungen getroffen werden. Die Regierung ist vom Vertrauen des Parlaments abhängig.

Die Repräsentativität resultiert aus der Tatsache, dass Parteien mit unterschiedlicher Programmatik sowie deren Mitglieder die verschiedenen Meinungen und Anschauungen der Bürger im Parlament vertreten.

In Repräsentativdemokratien wie dem parlamentarischen und präsidentiellen Regierungssystem übt das Volk die Herrschaft nicht die direkt aus, sondern es überträgt sie zauf Organe, die im Namen des Volkes die Regierungsgeschäfte wahrnehmen. ${ }^{1}$

Auf diese Weise ist ein hohes Maß an Meinungsfreiheit und Meinungsvielfalt als ein zentrales Merkmal der parlamentarischen Demokratie gewährleistet.

Weiterhin kennzeichnet das Vorhandensein einer Opposition den Pluralismus einer Demokratie; der Begriff der Demokratie könnte folglich um das Attribut parteienstaatlich erweitert werden. Der Pluralismus, verstanden als eine Vielzahl frei gebildeter politischer, wirtschaftlicher, religiöser, ethnischer und anderer Interessengruppen in Konkurrenz zueinander, gewährt einzelnen Bürgern oder Interessenverbänden größtmögliche Autonomie und politische Beteiligung; eine auf dem Pluralismus basierende vielgliedrige politische Ordnung ist offen für Konfliktentstehung und Konfliktaustragung einerseits sowie Konsensbildung andererseits. Da der Einzelne somit nicht einem einheitlichen Willen unterworfen ist, gewinnt er Entscheidungsfreiheit und Gestaltungsspielräume.

\subsection{Die Fragestellung}

Warum nun eine Untersuchung der politischen Sprachverwendung? Das wichtigste Werkzeug in der Politik ist die Sprache, denn

letztlich ist die Sprache das Instrument, mit dem Meinungen gedacht, erinnert, verbreitet und verändert, Dialoge geführt, Entscheidungen vorbereitet, begründet oder verteidigt werden, - und schließlich auch

1 Parlamentarische Demokratie I. In: Informationen zur politischen Bildung 227. Hg. v. Bundeszentrale für politische Bildung. Bonn ${ }^{32000, ~ S . ~} 5$ 
das Instrument, mit dem Haß erweckt, Androhungen von Gewalt und Angst verbreitet werden. ${ }^{2}$

Der Nachvollzug und die Beleuchtung der politischen Sprachverwendung als Werkzeug ist daher umso wichtiger und lohnender, je mehr eine Staatsform auf die Mündigkeit der Bürger einer Gesellschaft gebaut ist und je mehr die Macht verteilt ist. Die Reflexion der politischen Sprachverwendung kann in besonderem Maße durch die Analyse eines politischen Textes geschehen:

Bei einer Analyse wird oft nur bewusst gemacht, was ein gründliches intuitives Textverstehen spontan leistet. Der Vorzug des Analysierens gegenüber dem nur intuitiven Verstehen und spontanen Verwerten von Texten liegt nun aber darin, daß die Reflexion über den Text, über seine Wirkung, und der bewußte Einsatz von bewährten Analyseinstrumenten $\mathrm{zu}$ einer differenzierteren, objektiveren Erkenntnis führen, vor allem hinsichtlich der persuasiven Eigenschaften, die sonst meist nicht bewußt wahrgenommen werden. Eine solche Distanz vom unmittelbaren Textkonsum und ein solcher Zugewinn an wissenschaftlicher, kritischer Perspektive sind gerade im Bereich der politischen Meinungsbildung wertvoll, wenn nicht gar der einzige heute noch beschreitbare Weg zu einer humaneren politischen Praxis. ${ }^{3}$

Die Reflexion über die politische Sprachverwendung hilft folglich, die Bedingungen der politischen Kommunikation nachzuvollziehen, die sprachlichen Möglichkeiten der Inhaltsvermittlung zu erkennen und Kritikfähigkeit zu verbessern. Schließlich ist allein die Sprache als Träger der unterschiedlichsten Inhalte anzusehen, und

gerade in einer parlamentarischen Demokratie, in der die Konkurrenz politischer Gruppen und die Diskussion ihrer unterschiedlichen Vorstellungen ein wesentliches Merkmal der Politik ist, spielt die Sprache eine hervorgehobene Rolle, weil mit ihr auch unterschiedliche politische Programme und Zielvorstellungen erläutert werden. ${ }^{4}$

Die Sprache in der Politik ist weniger als Sondersprache zu verstehen; vielmehr stellt sie eine vielfältige Sprache dar, "die alle Bereiche des gesellschaftlichen Lebens durchdringt ${ }^{\prime \prime}$ und sich deren verschiedensten Aufgaben anpasst. Politische Sprache ist jedoch eng an die jeweilige Fachsprache geknüpft, die von den verwalteten Sachgebieten determiniert ist. Diese Behauptung wird in der folgenden Untersuchung zu überprüfen sein.

2 Bachem, Rolf: Einführung in die Analyse politischer Texte. München 1979, S. 11

3 Bachem 1979, S. 17

4 Bergsdorf, Wolfgang: Politik und Sprache. München 1978, S. 10

5 Bachem 1979, S. 13 


\subsection{Parteiprogramme: Spielarten und historische Entwicklung}

Die vorliegende Untersuchung beabsichtigt nun, solche Aspekte der politischen Sprache darzustellen und zu erläutern. Die Analyse wird sich dabei auf die politische Schriftsprache beschränken, wobei in diesem Zusammenhang in erster Linie die Grundsatzprogramme der Parteien zu nennen sind, wie bereits im Titel der Untersuchung angekündigt. Verwandte Texte mit programmatischem Charakter können exemplarisch hinzugezogen werden, wie beispielsweise Presseerklärungen, Depeschen, Regierungserklärungen, Bundestagsreden, Mitschriften von Bundestagsdebatten sowie Interviews; Reden oder politische Texte wie beispielsweise Zeitungskommentare können zwar ebenfalls programmatisch im Sinne von richtungweisend oder zielsetzend sein, sie haben aber eine jeweils verschiedene, von der Funktion von Parteiprogrammen eindeutig abgehobene Bedeutung.

Primär liegen dieser Analyse jedoch schriftlich fixierte Texte zugrunde, die offiziellen und grundsätzlichen Charakter haben und sich aus verschiedenen, den Parteien zugrunde liegenden politischen Ideologien ergeben. Dabei stehen Texte des 20. Jahrhunderts im Vordergrund, es soll jedoch auch der Versuch unternommen werden, geschichtliche Entwicklungstendenzen nachzuzeichnen oder zumindest die aus der Betrachtung der Programme der Gegenwart gewonnenen Erkenntnisse mit den Verwendungsweisen in Parteiprogrammen des 19. Jahrhunderts zu vergleichen, "da sich [...] das So-Sein der Gegenwart allein dem SoGewesensein der Vergangenheit verdankt und daher auch die gegenwärtige politische Kommunikation nur aus der vergangenen erklärt werden kann $[\ldots] .{ }^{\prime \prime} 6$

Trotz der Einschränkung auf Grundsatzprogramme einzelner Parteien sollen auch Textbeispiele aus Wahlprogrammen exemplarisch miteinander verglichen werden. Dabei liegt der Untersuchungsschwerpunkt weniger auf den jeweiligen Inhalten der Parteiprogramme als vielmehr auf der sprachlichen Gestalt der Texte und ihrer Verwendung zur Vermittlung der Inhalte. Es geht in dieser Untersuchung folglich um die Beleuchtung der Sprache im Kontext politischer Sprachhandlungen; BURKHARDT (1996) favorisiert die als paronomastische Kontamination aus Politologie und Linguistik zu verstehende Bezeichnung Politolinguistik

Burkhardt, Armin: „Politolinguistik - Versuch einer Ortsbestimmung.“ In: Sprachstrategien und Dialogblockaden. Linguistische und politikwissenschaftliche Studien zur politischen Kommunikation. Hg. v. Josef Klein/Hajo Dieckmannshenke. Berlin/New York 1996, S. 88 
für diese Disziplin der Sprachwissenschaft; auf die erwähnten Teilbereiche wird zu einem späteren Zeitpunkt eingegangen:

Für die bisher namenlose Disziplin der Sprachwissenschaft, die sich mit der Untersuchung der politischen Sprache im allgemeinen und deren [...] Teilbereichen im besonderen beschäftigt, möchte ich die Bezeichnung Politolinguistik vorschlagen. ${ }^{7}$

Um dabei einen gemeinsamen Nenner zu finden soll sich die Untersuchung soweit möglich auf Parteiprogramme beschränken. In diesem Zusammenhang stellen sich zentrale Fragen: Wie werden Anschauungen und Überzeugungen in der Politik dargeboten? Mit welchen sprachlichen Mitteln wird ein Grundsatz formuliert und ein politischer Text gestaltet?

\subsubsection{Das Parteiprogramm als Textsorte}

Politische Parteien können als Vereinigungen von Bürgern und deren Repräsentanten betrachtet werden; die Politiker als Repräsentanten werden in einer Demokratie durch Wahlentscheidungen beauftragt. Da Parteien „dauernd oder für längere Zeit für den Bereich des Bundes oder eines Landes auf die politische Willensbildung Einfluß nehmen und an der Vertretung des Volkes im Deutschen Bundestag oder einem Landtag mitwirken wollen“8, müssen sie den politischen Willen der Bürger artikulieren und in ihre Ziele integrieren. Im Idealfall geschieht dies mit der Konzeption eines Parteiprogramms; allerdings wachsen die Wünsche der Wähler und der Parteimitglieder nicht einfach zu einem Parteiwillen oder einem Parteiprogramm zusammen; "die oft durchaus amorphe Masse der Wünsche und Anregungen konturiert sich nicht von selbst zu in sich stimmigen Konzeptionen. ${ }^{.9}$

Erarbeitet und formuliert werden Parteiprogramme von Programmkommissionen, die durch die Vorstände der Parteien eingesetzt werden: „Wo ein Programm vorzubereiten ist, werden in allen Parteien ad hoc Kommissionen berufen, die mit der Erledigung ihres Auftrags ihre Tätigkeit einstellen. " ${ }^{10}$ Ein Parteiprogramm wird folglich von einer Gruppe hochqualifizierter Personen unter Ausschluss der Öffentlichkeit entwor-

\section{Burkhardt 1996, S. 82}

8 Bundestagswahl 1980. Die Parteien und ihre Programme. In: Informationen zur politischen Bildung 185. Hg. v. Bundeszentrale für politische Bildung. Bonn 1980, S. 7

9 Flohr, Heiner: Parteiprogramme in der Demokratie. Ein Beitrag zur Theorie der rationalen Politik. Göttingen 1968, S. 139

10 Eppler, Erhard: Grundwerte für ein neues Godesberger Programm. Hamburg 1984, S. 7 
fen; diese Tatsache sollte bei der Betrachtung der Sprachverwendung in den folgenden Kapiteln immer mitberücksichtigt werden. Die Kommission muss für das Gelingen des Parteiprogramms in ein reges Verhältnis des Meinungsaustausches mit möglichst vielen Parteimitgliedern treten und die zahlreichen und vielfältigen Meinungsäußerungen koordinieren. Die Beteiligung der breiten Masse der Parteimitglieder kann sich allerdings lediglich auf zwei Weisen vollziehen:

Erstens können die Mitglieder gleich am Anfang beantragen, bei der Programmierung bestimmte Ziele zu setzen, oder auf andere zu verzichten; zweitens können sie - in verschiedenen Stadien der Arbeit am Programm - Vorschläge der Programmkommission prüfen und zu ihnen Stellung nehmen, auch indem sie die Programmkommission mit bestimmten Änderungen des Entwurfs beauftragen. ${ }^{11}$

Bei der Akzeptanz der Mehrheit der Parteimitglieder wird der von der Kommission vorgelegte Entwurf durch eine formelle Annahme zum Programm erhoben.

\subsubsection{Das Parteiprogramm als Sprachspiel}

Parteiprogramme sind Texte, die in politischen Willensbildungs- und Entscheidungsprozessen eine zentrale Rolle spielen. Die Kombination aus Partei(mitgliedern) als Autorschaft, der Adressatengruppe, des Themenbezugs eines jeweiligen Programms und der beabsichtigten Textfunktion ergibt einen konkreten Interaktionszusammenhang bzw. ein spezielles Sprachspiel:

Sprachspiele sind Teile einer bestimmten sozialen Wirklichkeit, in denen die allgemeinen Strukturen von Kommunikationsprozessen - also kommunikative Verfahren - spezifisch eingesetzt werden. ${ }^{12}$

Der Sprachspielbegriff stellt sich daher als konkrete Handlungs-, Aufgaben-, Problemlösungs- oder Interaktionskonstellation dar; es konstituiert sich als Konstellation von

spezifischen Problemsituationen, Handlungsbeteiligten mit bestimmten (sozialen) Beziehungen zueinander (Funktionen, Rollen), gemeinsamem Situations- und Hintergrundwissen der Kommunikationspartner, Aufgabenstellungen und Handlungszielen (bei Sachverhalten mit bestimmten Problemstrukturen), kommunikativen Strategien, allgemeinen Herrschaftsbedingungen, gesellschaftlichen Organisations-

$11 \quad$ Flohr 1968, S. 138

12 Strauß, Gerhard: „Sprachspiele, kommunikative Verfahren und Texte in der Politik. Versuch einer Textsortenspezifik." In: Der politische Wortschatz. Zur Kommunikations- und Textsortenspezifik. Hg. v. Gerhard Strauß. Tübingen 1986a, S. 33 
formen, beteiligten Institutionen und Instanzen (institutionellen Rahmenbedingungen). ${ }^{13}$

Wieso aber wird der Ausdruck Sprachspiel benutzt? Es gibt eine Tradition, die Sprache mit Spielen zu vergleichen; der Ausdruck des Sprachspiels ist ein aus der analytischen Philosophie und Sprachphilosophie stammender Begriff und geht auf WITTGENSTEIN (1977) zurück:

[...] Ich werde auch das Ganze: der Sprache und der Tätigkeit, mit denen sie verwoben ist, das ,Sprachspiel' nennen. (\$7)

Das Wort ,Sprachspiel' soll hier hervorheben, daß das Sprechen der Sprache ein Teil ist einer Tätigkeit, oder einer Lebensform. $(\$ 23)^{14}$

So wie ein Spiel durch bestimmte Regeln als normierende Festlegungen charakterisiert wird ist - dieser Spieltheorie folgend - auch ein Sprachspiel durch die kommunikativen Verfahren charakterisiert, die diesem zugrunde liegen. Die kommunikativen Verfahren wiederum sind als Prozesse bzw. komplexe Aktivitäten zu verstehen:

Sprachliche Handlungen sind wie Spielhandlungen von Regeln bestimmt. Ob eine Handlung als diese oder jene zählt, wird durch die Regeln des Spiels bestimmt. Spielhandlungen sind wie sprachliche Handlungen auf Verstehen angelegt [...]. Und Verständnis ist jeweils nur so weit möglich, wie die Handlung gemäß der gleichen Regel zu verstehen ist. Dazu müssen wir ihre Stellung im ganzen Spiel beachten. ${ }^{15}$

Texte, folglich auch Parteiprogramme, sind die Produkte, die aus diesen Prozessen hervorgehen: "Texte sind die statischen Produkte eines bestimmten Sprachspiels (als konkreter Interaktionszusammenhang) in Realisierung eines bestimmten kommunikativen Verfahrens, das in diesem Sprachspiel ,mitspielt'. "16 Die konkreten Sprachspiele und die einhergehenden kommunikativen Verfahren der jeweiligen Programmtypen werden zu einem späteren Zeitpunkt definiert und erläutert; das nachfolgende Zitat soll die Theorie des Sprachspiels abrunden: „Mithilfe von Sprachspielen kann so ein relativ realistisches und exaktes Bild der Wirklichkeit politischer Interaktion in einer bestimmten Gesellschaft entworfen werden." ${ }^{17}$

13 Strauß 1986a, S. 5

14 Wittgenstein, Ludwig: Philosophische Untersuchungen. Frankfurt/Main 1977, S. $19 / 28$

15 Heringer, Hans Jürgen: „Sprachspiele und ihre Geschichte.“ In: Einführung in die Praktische Semantik. Hg. v. Hans Jürgen Heringer/Günther Öhlschläger/Bruno Strecker/Rainer Wimmer. Heidelberg 1977, S. 67/68

16 Strauß 1986a, S. 35

17 Strauß 1986a, S. 6 
An dieser Stelle soll auf EDELMAN (1976) und dessen Theorie der Doppelung hingewiesen werden. Da einem Sprachspiel wie beispielsweise einem Grundsatzprogramm als politische Textsorte verschiedenartige, teilweise widersprüchliche kommunikative Verfahren zugrunde liegen, ergibt sich daraus eine Doppelung; diese resultiert aus der Tatsache, dass politisches Handeln sowohl in der breiten Öffentlichkeit als auch in Teilöffentlichkeiten bestimmter Institutionen wie Parteien, Fraktionen oder Verbände stattfindet. Auf dieser Ansicht, dass politisches Handeln ",sowohl ein Durchsetzungs- wie ein Inszenierungsproblem“ ist, basiert die Theorie der "Realitätsdoppelung“: Die Realität des Politischen ist eine Doppelung, „eine Brechung in zwei Realitätsebenen: Machtkampf und Täuschung über diesen Machtkampf, Nachricht und Deutung, strategische Rationalität und symbolische Mystifikation." ${ }^{18}$ Der politischen Sprachverwendung als Willensbildungs- und Entscheidungsprozesse lassen sich danach zwei Funktionsweisen attestieren:

die eine auf der Ebene der (direkten) Interaktion der politischen Akteure, die andere auf der Ebene der Beziehung zwischen den politischen Akteuren und der zuschauenden und nur indirekt beteiligten Öffentlichkeit. ${ }^{19}$

Der Brechung in zwei unterschiedliche Bedeutungsebenen unterliegt auch das politische Handeln, da dieses überwiegend kommunikatives bzw. sprachlich vermitteltes Handeln ist:

Einerseits hat kommunikatives Handeln eine Bedeutung in der Interaktion der politischen Akteure bei der Lösung von Problemen der Handlungskoordination, andererseits macht der zuschauende oder zuhörende Bürger sich aus dem kommunikativen Handeln der Akteure bzw. aus dem massenmedial vermittelten Handeln eine Vorstellung davon, auf welche Art die Probleme der Handlungskoordination gelöst werden. ${ }^{20}$

Da sich die politischen Akteure über die oben dargestellte zweite Bedeutungsebene permanent bewusst sind (d.h. die Meinung des Rezipienten über die politischen Akteure basiert auf deren sprachlichen und tatsächlichen Handlungen) und sie daher ihre Handlungen immer an den unterschiedlichen Möglichkeiten der Rezeption orientieren, können das politische Geschehen und die sprachlichen Handlungen als Inszenierung betrachtet werden. Das auf der ersten Ebene präsentierte politische Geschehen wird für den Bürger/Wähler in einer Weise inszeniert, „daß er

18 Offe, Claus: „Editorial.“ In: Murray Edelman: Politik als Ritual. Die symbolische Funktion staatlicher Institutionen und politischen Handelns. Frankfurt/Main 1976, VIII

19 Strauß 1986a, S. 14

20 Strauß 1986a, S. 14 
nicht in der Lage ist zu erkennen, was wirklich geschieht oder welche Ziele in Wirklichkeit verfolgt werden, sondern einer Täuschung oder ,Mystifikation' aufsitzt.“" ${ }^{21}$

Die dargestellten Zusammenhänge wirken sich auf vielfältige Weise auf die Konzeption eines Parteiprogramms aus. Bei der Untersuchung der sprachlichen Handlungen und des verwendeten Vokabulars des 1959 von der SPD verabschiedeten Godesberger Programms und der anderen Parteiprogramme müssen solche Zusammenhänge stets mitberücksichtigt werden.

Grundsätzlich versteht man unter dem Ausdruck Parteiprogramm

die in einem einheitl[ichen] Dokument festgelegte Zusammenfassung der Grundprinzipien und Ordnungsvorstellungen einer Partei, [das] vom Parteitag meist für eine nicht begrenzte Zeit verabschiedet [wird]. ${ }^{22}$

Diese Definition gibt allerdings eine lediglich grobe Orientierung; nach ihr ist ein Parteiprogramm als Grundsatzprogramm mit zeitlich unbegrenzter Gültigkeitsdauer zu verstehen. Die Parteien haben jedoch kein allumfassendes Programm, sondern differenzieren zwischen verschiedenen Typen, die jeweils unterschiedlich konzipiert sind. So gibt es neben dem Grundsatzprogramm auch zeitlich befristete Parteiprogramme, so genannte Wahl- oder Aktionsprogramme, die zu aktuellen und spezielleren Themen Bezug nehmen und die in der Regel vor Bundestags-, Landtags- oder Kommunalwahlen von den Parteien auf der jeweiligen politischen Ebene veröffentlicht werden.

$\mathrm{Da}$ in der folgenden Untersuchung die unterschiedlichen Typen von Parteiprogrammen ohne weitere Erklärung oder Definition an der jeweiligen Stelle verwendet werden, soll nachfolgend eine Spezifizierung dieser Programmtypen erfolgen, um die späteren Verwendungen der Begrifflichkeiten nachvollziehbar zu machen. Eine eingehende Darstellung und Untersuchung der Funktionen dieser Programmtypen wird auf das spätere Kapitel Pragmatische Aspekte der Textorganisation verschoben.

\subsubsection{Wahl-/Aktionsprogramme}

Wahl- oder Aktionsprogramme stehen oftmals unter einem bestimmten und aus der jeweiligen Zeitgeschichte resultierenden Motto und sind für einen kurzfristigen Zeitraum konzipiert, etwa den einer Legislaturperiode (bis zu fünf Jahren); daher konzentriert sich dieser Programmtyp lediglich auf die Politik in diesem Zeitraum. Eine detaillierte Zeitanalyse

21 Strauß 1986a, S. 15

22 Brockhaus. Die Enzyklopädie. Band 6. Leipzig/Mannheim 201998, S. 602 
wird sich jedoch in einem Aktionsprogramm kaum finden lassen; die jeweiligen Ziele werden nur genannt, nicht begründet.

Auch das Votum des Wählers gilt lediglich für den unmittelbar bevorstehenden und überschaubaren Zeitabschnitt. Demzufolge haben Wahlprogramme in erster Linie die Aufgaben bzw. die Handlungsziele, Meinungen in bestimmter Weise $\mathrm{zu}$ beeinflussen, potentielle Wähler zu überzeugen, $\mathrm{zu}$ überreden oder zu werben und dadurch zu gewinnen. Weiterhin haben sie zum Ziel, Anleitungen und Argumentationshilfen für den Wahlkampf der eigenen Parteimitglieder zu bieten und die eigene Position gegenüber anderen Parteien abzugrenzen. Diese Ziele resultieren letztlich aus den Bemühungen, Macht durch eine parlamentarische Mehrheitsbildung zu gewinnen.

Aufgrund der Werbefunktion kann das Wahlprogramm als Sprachspiel Politische Werbung verstanden werden. Die Bilanz der gegenwärtigen Situation einer Partei ist daher als ein wichtiges Werkzeug von Wahlprogrammen anzusehen; Parteien der Opposition werden dabei bestrebt sein, Alternativen zu bieten, Parteien der Regierung hoffen auf eine Wiederwahl und halten unter Umständen an ihrer Richtung fest, möglicherweise jedoch mit Modifikationen (Reformen). Somit lassen sich Parteien, Interessengruppen und Verbände als Institutionen bzw. Instanzen benennen, die auf das Wahlprogramm als Text einwirken.

Wahlprogramme sind oftmals detailliert und präzise formuliert und resultieren aus den Positionen und Inhalten der Grundsatzprogramme: „Wahlprogramme enthalten in einer für die breite Öffentlichkeit übersetzten, leicht verständlichen Sprache Aussagen, die mit der geltenden Parteidoktrin (festgelegt in den Parteiprogrammen) und Parteiideologie übereinstimmen. “23 Das Wahlprogramm ist daher als konkretes Programm zu verstehen; dieser Programmtyp,

vor allem vor Wahlen oder entscheidenden politischen Abschnitten aufgestellt, bringt für die Parteien eine flexiblere Operationsbasis, schafft eine stärkere Anpassungsfähigkeit und die Möglichkeit der ständigen Orientierung am Wählerverhalten und Wählerwillen. ${ }^{24}$

Das Wahlprogramm richtet sich als Adressatengruppe an die breite Öffentlichkeit (Volkspartei) oder einzelne Zielgruppen (Randpartei); die Bürger als Massenöffentlichkeit und als Empfängerkollektiv können daher durchaus als indirekt oder passiv Beteiligte an der Entstehung des Wahlprogramms betrachtet werden, da deren mögliche Rezeption bereits während dessen Konzeption berücksichtigt wird. Daher „formulieren die Parteien und Interessenverbände [...] ihre Beiträge zur Normen-

23 Strauß 1986a, S. 54

24 Kaack, Heino: Geschichte und Struktur des deutschen Parteiensystems. Opladen 1971, S. 401 
diskussion (Regierungserklärungen, Wahlkampfprogramme, Grundsatzpapiere etc.) in stärkerem oder schwächerem Maße als werbende Botschaften. ${ }^{25}$ Folglich finden sich Wahlprogramme in politischen Broschüren, in komprimierter Form als Flugblätter oder auszugsweise in Zeitungen, Zeitschriften oder Illustrierten abgedruckt. Meinungsforschungsinstitute können ebenfalls als sekundär an der Konzeption Beteiligte betrachtet werden, da deren Prognosen die derzeitigen Stimmungsbilder der Bevölkerung als Massenwählerschaft wiedergeben; „wissenschaftliche Methoden, wie die Methoden der Meinungsbefragung [...] erlauben den Parteien eine weitgehende Zukunftsorientierung ihrer Planung. ${ }^{26}$ Das Wahlprogramm geht mit seinem Zeitbezug daher auf solche Prognosen ein. Direkt oder aktiv an der Entstehung Beteiligte sind die politische Partei bzw. Gruppe und deren Angehörige, aber auch Interessenverbände oder Bürgerinitiativen.

Aufgrund dieser Voraussetzungen ist das Wahl- oder Aktionsprogramm als das strategischste oder erfolgsorientierteste politische Sprachspiel zu verstehen.

\subsubsection{Orientierungsprogramme}

Daneben verabschieden Parteien Programme, die sowohl grundsätzliche als auch konkrete Aussagen von mittelfristiger Geltungsdauer enthalten; diese so genannten Orientierungsprogramme sind für Zeiträume von fünf bis fünfzehn Jahren konzipiert, wie beispielsweise der Orientierungsrahmen ' 85 der SPD oder die Mannheimer Erklärung der CDU. Orientierungsprogramme bieten detaillierte Ausformulierungen von mittelfristigen politischen Zielen; zwar werden solche Programme ebenso wie die beiden anderen Kategorien der Öffentlichkeit auf Bundesparteitagen vorgestellt, aber "die hauptsächliche Funktion dieses Programmtyps liegt seiner Zwecksetzung entsprechend darin, Handlungsanleitung für die in Regierungspositionen stehenden Parteimitglieder zu sein. “27 Hinsichtlich der Adressatengruppe richtet sich dieser Programmtyp folglich sowohl an die Gesellschaft allgemein als breite Öffentlichkeit als auch an die Parteiführung und deren Mitglieder als Teilöffentlichkeit.

\subsubsection{Grundsatzprogramme}

Beide bereits erwähnten Kategorien sind von Regierungs- oder Grundsatzprogrammen abzugrenzen, die eher allgemeingültigen Charakter haben und ihre Geltung über Zeiträume hinweg besitzen;

25 Bachem 1979, S. 14

26 Kaack 1971, S. 401/402

27 Bundestagswahl 1980. Die Parteien und ihre Programme 1980, S. 10 
"Grundsatzprogramme wirken sowohl in die Parteien hinein als auch nach außen. Sie legen die Grundsätze fest, die den ideologischen Rahmen einer Partei darstellen. " ${ }^{28}$ Folglich geht ein Grundsatzprogramm aus einem innerparteilichen Meinungs- und Willensbildungsprozess hervor und kann daher als Sprachspiel gruppenbezogene oder parteiinterne politische Meinungsbildung gelten. Das Grundsatzprogramm dient der Selbstfindung einer Partei und legt die leitenden und verpflichtenden Prinzipien fest. Somit lassen sich die politischen Parteien oder Interessenverbände als Institutionen bzw. Instanzen benennen, die auf das Grundsatzprogramm als Text einwirken.

Das Grundsatzprogramm einer Partei legitimiert das Handeln ihrer Mitglieder, die sich darauf berufen; weiterhin dient es der Integration der Parteimitglieder und der innerparteilichen Gruppierungen.

Die jeweiligen Ziele werden im Grundsatzprogramm nicht nur genannt, sondern auch begründet; im Gegensatz zum Aktionsprogramm werden hier weiterhin zeitanalytische Ausführungen zu finden sein. Da ein Grundsatzprogramm für einen längerfristigen Zeitrahmen konzipiert ist, wird es sich nicht wie das Wahlprogramm mit aktuellen Dingen befassen und Stellungnahmen zu aktuellen oder speziellen politischen Fragen bieten, sondern es wird notwendigerweise allgemeiner gehalten sein. Von Zeit zu Zeit bedarf jedoch auch das Grundsatzprogramm aufgrund der sich verändernden gesellschaftlichen Bedingungen der Überholung. Die NSDAP erhebt in ihrem Programm vom 24. Februar 1920 allerdings einen zeitunabhängigen Geltungsanspruch ohne Berücksichtigung eines möglichen Wandels der gesellschaftlichen Bedingungen:

(a) Das Programm der Deutschen Arbeiterpartei ist ein ZeitProgramm. Die Führer lehnen es ab, nach Erreichung der im Programm aufgestellten Ziele neue aufzustellen, nur zu dem Zwecke, um durch künstlich gesteigerte Unzufriedenheit der Massen das Fortbestehen der Partei zu ermöglichen. (NSDAP 1920, S. 15)

Das Grundsatzprogramm zielt primär auf die Parteiführung und deren Mitglieder und sekundär auf politisch besonders interessierte Bürger als Teilöffentlichkeit ab. Im Gegensatz zum Wahlprogramm mit den darin formulierten konkreten Aussagen spielt die Öffentlichkeit als Adressatengruppe beim Grundsatzprogramm daher kaum eine Rolle.

Die dargestellten drei Parteiprogrammtypen ergänzen sich gegenseitig; so ist das an mancher Stelle abstrakt und allgemeingültig wirkende Grundsatzprogramm darauf angewiesen, durch die Wahlprogramme präzisiert zu werden, einen Zeitbezug zu bekommen oder in einen gesellschaftspolitischen Diskurs eingebettet zu werden. Das Grundsatzprogramm darf in den Formulierungen die Partei nicht einengen, da die

28 Bundestagswahl 1980. Die Parteien und ihre Programme 1980, S. 10 
Flexibilität und der Handlungsspielraum für die Aktionsprogramme gewahrt bleiben muss:

Die Grundsatzprogramme müssen so allgemein gehalten und damit im Grunde genommen unverbindlich sein, daß sie für längere Zeit gültig bleiben, aber dennoch offen sind für Veränderungen in den strukturellen Grundbedingungen der Politik und in konkreten politischen Entscheidungen. ${ }^{29}$

Konkretisiert werden die Inhalte der Grundsatzprogramme durch die Wahlprogramme; diese fußen mit ihrer kurzfristigen Geltungsdauer auf den im Grundsatzprogramm verankerten Glaubensbekenntnissen der Partei. Auf der Basis eines Wahlprogramms werden die Inhalte des jeweiligen Grundsatzprogramms durch die Massenmedien der Öffentlichkeit bekannt gemacht. Als Werbeträger spielen die Massenmedien daher in der Beurteilung von Parteiprogrammen eine wichtige Rolle.

Problematisch wird das Wechselverhältnis zwischen Wahlprogramm und Grundsatzprogramm allerdings dann, wenn bestimmt Maßnahmen zwar die Ziele des Wahlprogramms erfüllen, aber unvereinbar mit den grundsätzlichen und langfristigen Zielen des Grundsatzprogramms (beispielsweise Maßnahmen eines Wahlprogramms, die zu höheren Ausgaben und damit stärkeren Belastungen des nächsten Etats führen und die mit den Zielen des Grundsatzprogramms eigentlich unvereinbar sind).

Grundsatzprogramme sind beispielsweise die Freiburger Thesen der FDP oder das Godesberger Programm der SPD; um letzteres soll es in der folgenden Untersuchung zunächst gehen.

\subsection{Historische Hintergründe des Godesberger Programms}

Primäre Textgrundlage für eine detaillierte Analyse ist neben anderen das Grundsatzprogramm der Sozialdemokratischen Partei Deutschlands $(\mathrm{SPD})^{30}$ von 1959 in ungekürzter Fassung, zitiert aus Parteiprogramme von Siegfried Hergt. Dieses Parteiprogramm ist auch bekannt als Godesberger Programm und wird daher in der folgenden Analyse als solches bezeichnet (die Bezeichnung wird auch in dieser Form als Referenz verwendet, bei anderen Parteiprogrammen werden die jeweilige Partei und die Jahreszahl angegeben).

29 Kaack 1971, S. 402

30 Hergt, Siegfried (Hrsg.): Parteiprogramme. SPD, CDU, CSU, FDP, DKP, NPD. Eine Dokumentation der Grundsatzprogramme und aktueller politischer Ziele. Opladen ${ }^{7} 1975$, S. $68-87$ 
Das Godesberger Programm soll in diesem Kapitel ausführlich in einen historischen Kontext eingebettet werden, denn dessen Annahme und Verabschiedung hat

viel zu einer innerparteilichen Beruhigung und Klärung, vor allem aber zu einem Wandel des Erscheinungsbildes der SPD in der Öffentlichkeit beigetragen. Damit war die Voraussetzung geschaffen, das von ihr angestrebte Ziel erreichen zu können: eine von verschiedenen Schichten wählbare ,Volkspartei' zu werden. ${ }^{31}$

Ein außerordentlicher Parteitag der SPD vom 13.-15. November 1959 in Bad Godesberg (heute ein Stadtteil von Bonn) verabschiedet ein neues Grundsatzprogramm, das die seit 1957 laufenden Diskussionen um ein neues Programm der Partei kanalisieren sollte. Das bisher gültige Heidelberger Programm von 1925 sowie die vorhergehenden Aktionsprogramme von 1952 und 1954 wurden dadurch abgelöst.

Die Vorstandswahlen auf dem Stuttgarter Parteitag von 1958 sind als Ausdruck eines tief greifenden Wandels zu verstehen, der sich in der SPD seit dem Tod von Kurt Schumacher 1952 vollzog. Unter den elf neu gewählten Mitgliedern des SPD-Vorstands befanden sich Willy Brand, Helmut Schmidt und Gustav Heinemann. Herbert Wehner gehörte dem Bundestag von 1949 bis 1983 an, von 1958 bis 1973 war er stellvertretender Bundesvorsitzender der SPD; "als führender Parteistratege war er maßgeblich an der Gestaltung und Durchsetzung des Godesberger Programms und an der Umwandlung der SPD von einer Klassen- in eine linke Volkspartei beteiligt. “32

Der historische Hintergrund des Godesberger Programms ist mehr als dessen bloßer geschichtlicher Kontext. Die politische Lage und die geschichtliche Entwicklung sind vielmehr als Entstehung und Wirkungsbedingung des Programms anzusehen, die zwangsläufig Auswirkungen sowohl auf die thematische Vielfalt als auch auf die Formulierungen haben. Um die Einordnung des Godesberger Programms in den polithistorischen Zusammenhang zu ermöglichen, soll dieser zunächst kurz umrissen werden.

Die sozialen, ökonomischen und internationalen Rahmenbedingungen in der Bundesrepublik Deutschland hatten sich im Verlauf der fünfziger Jahre des 20. Jahrhunderts grundlegend verändert. Das liberalkonservative Modell der Regierung mit Konrad Adenauer als erstem Bundeskanzler der BRD bewirkte einen Wirtschaftsaufschwung, der unerwartet kam und von dem in zunehmendem Maße auch die Arbeiterschaft profitieren konnte. Das so genannte "Wirtschaftswunder" und

31 Miller, Susanne: Die SPD vor und nach Godesberg. Bonn 1975, S. 38

32 Müller, Helmut M.: Schlaglichter der deutschen Geschichte. Mannheim ${ }^{3} 1996$, S. 378 
der damit einhergehende breitgestreute Wohlstand in allen Gesellschaftsschichten führten dazu, dass die politische Idee des Sozialismus an Attraktivität verlor und neu überdacht werden musste. Diese Entwicklung wurde zusätzlich beschleunigt durch das Wirtschaftssystem der DDR, das sich auf den Sozialismus berief, im Gegensatz zur BRD jedoch lediglich die Grundbedürfnisse der Bevölkerung befriedigen konnte und dadurch zunehmend als Negativbeispiel verstanden wurde. Die Wahlniederlagen in den Jahren 1953 und 1957 zeigen die relative innenpolitische Erfolglosigkeit der SPD in den fünfziger Jahren.

Weiterhin verlagerte sich der Schwerpunkt des politischen Handelns in der BRD von der Innenpolitik auf den Bereich der Außenpolitik. Vor allem der deutsche Sozialismus stand damit vor neuen Herausforderungen. Die SPD sah sich unter diesen Umständen gezwungen, eine eigene Programmatik zu entwickeln, in der die politischen Überzeugungen zeitgemäß und deutlich formuliert sind. Insbesondere die Fragen der Eingliederung der BRD in die Europäischen Gemeinschaften, des Eintritts Deutschlands in die Nordatlantische Verteidigungsgemeinschaft (NATO) sowie die Frage nach der Wiederbewaffnung einschließlich der Stationierung von Atomwaffen auf deutschem Boden verlangten klare Stellungnahmen von der SPD als Oppositionspartei. Daher sah sich die SPD veranlasst,

die Grundüberzeugungen des Sozialismus zu überdenken, mit dem Ziel, eine angemessene und zeitgerechte Analyse der Gesellschaft der Gegenwart zu erarbeiten, daraus die richtigen Folgerungen abzuleiten und damit den Sozialismus mehrheitsfähig werden zu lassen. Ihren Höhepunkt erreichten diese Bemühungen im Godesberger Programm aus dem Jahre 1959, mit dem eine neue pragmatische Politik der Partei begann. ${ }^{33}$

Das Godesberger Programm und die damit verbundene Wandlung der Partei erschloss neue Wählerschichten und legte auf diese Weise die Basis für die späteren Wahlerfolge der SPD, die bei den Bundestagswahlen von 1965 39,3 Prozent der Wählerstimmen erzielen konnte; weiterhin machte das Grundsatzprogramm die SPD für die bisherigen Regierungsparteien CDU/CSU und FDP koalitionsfähig. Mit dem Eintritt der SPD in die große Koalition im Jahr 1966 hatte sich die im Godesberger Programm angelegte Strategie bewährt; auf dieser Grundlage entwickelte die SPD ihre Programmatik weiter und veröffentlichte im Juni 1987 einen Entwurf für ein neues Grundsatzprogramm, welches das Godesberger Programm von 1959 ablöste; diese Zeitspanne verdeutlicht den Gültigkeitsanspruch eines Grundsatzprogramms. Dieses zweite Grundsatz-

33 Politische Ideologien. In: Informationen zur politischen Bildung 212. Hg. v. Bundeszentrale für politische Bildung. Bonn ${ }^{2} 1990$, S. 17 
programm der SPD wurde dann erst wieder am 17.04.1998 auf dem Parteitag in Leipzig geändert und ist in dieser Fassung bis heute gültig.

Grundsatzprogramme anderer einflussreicher deutscher Parteien werden ergänzend oder zur Abgrenzung hinzugezogen. Das Godesberger Programm ist als Grundsatzprogramm einer Volkspartei anzusehen. Unter Volksparteien versteht man allgemein solche Parteien, "die sich in ihren Aktivitäten grundsätzlich an sämtliche sozialen Gruppen und gesellschaftlichen Schichten wenden. “ ${ }^{34}$ So bezeichnet sich die CDU in ihrem Programm von 1971 und die SPD im Jahre 1989 explizit als Volkspartei (vgl. 33 (d) und 6 (b)). Die Entwicklung einer Randpartei zur Volkspartei zieht dabei bestimmte Auswirkungen nach sich, die sich zwangsläufig in der Programmatik niederschlagen:

Der wachsende Konsensus in prinzipiellen politischen Fragen sowie der sich daraus und aus anderen Ursachen ergebende Trend zu sogenannten Volksparteien verminderten die ehedem teilweise krassen Gegensätze zwischen den Parteien. Folge und zugleich Symptom dieses Angleichungsprozesses ist die zunehmende Ähnlichkeit der Parteiprogramme. Dem Wähler ist weniger als früher möglich, anhand der Parteiprogramme eine begründete Entscheidung für eine Partei zu treffen. ${ }^{35}$

Die Behauptung, dass die Programme der Parteien zunehmend weniger Unterschiede aufweisen, leuchtet ein, wird im Folgenden jedoch ebenso zu überprüfen sein wie die Vermutung, auf Basis der Programme könne der Wähler keine rationalen Wahlentscheidungen mehr treffen, da die Parteiprogramme keine begründeten Wahlentscheidungen mehr ermöglichten. Daher sollen Parteiprogramme kleinerer Randparteien nach Möglichkeit ebenfalls in die Analyse eingebunden werden, um ein möglichst breites Spektrum abzubilden. Untersucht werden soll hierbei, ob die Verwendungsweisen von einzelnen Wörtern oder Formulierungen sowie die Inhaltsvermittlung parteispezifisch und lediglich für das Godesberger Programm typisch sind oder ob allgemeingültige Muster erkennbar sind, die charakteristisch für das Parteiprogramm als gattungsspezifische Textsorte sind.

Die stilistisch signifikanten linguistischen Merkmale eines Textes können jedoch oft genauer beobachtet und eindrücklicher dargestellt werden, wenn ein expliziter Vergleich mit einem anderen Text unternommen wird oder als ,Kontrolle' während der stilistischen Analyse dient. [...] Die Texte, die vergleichend und/oder kontrollierenden Zwecken dienen, sollten selbstverständlich weder zu ähnlich (es sei denn, es ginge um ,Gruppenstile') noch zu unähnlich sein: im ersten Fall kämen lediglich bei einem hypothetischen Grad der äußersten

34 Bundestagswahl 1980. Die Parteien und ihre Programme 1980, S. 9

35 Flohr 1968, S. 37 
Feinheit aufschlußreiche kontrastive, linguistische Merkmale zum Vorschein; im zweiten Fall wären die Unterschiede so grob, daß eine ausführliche Analyse müßig wäre. ${ }^{36}$

Folgende Parteiprogramme werden in dieser Untersuchung aufgenommen:

- das Eisenacher Programm - Das Programm der Sozialdemokratischen Arbeiterpartei (SDAP), 186937;

- das Gothaer Programm - Das Programm der Sozialistischen Arbeiterpartei Deutschlands (SAP), $1875^{38}$;

- das Programm der Kommunistischen Partei Deutschlands (KPD), 1918/1939;

- die Grundsätze der Deutschen Volkspartei (DVP), $1919^{40}$

- das Grundsatzprogramm der Nationalsozialistischen Deutschen Arbeiterpartei (NSDAP), 192041;

- das Grundsatzprogramm der Deutschnationalen Volkspartei (DNVP), 192042;

- der Gründungsaufruf der Konservativen Volkspartei, 193043;

- die Richtlinien des Zentrums, 195144;

- das Programm der Sozialistischen Einheitspartei Deutschlands (SED), 19634;

- das Grundsatzprogramm der Christlich-Sozialen Union (CSU), 196846;

- die Grundsatzerklärung der Deutschen Kommunistischen Partei (DKP), 196947;

36 Spencer, John und Gregory, Michael: „Eine Stellungnahme zum Sprachstil.“ In: Linguistik und Stil. Hg. v. John Spencer. Heidelberg 1972, S. 87

37 Mommsen, Wilhelm (Hrsg.): Deutsche Parteiprogramme. München 1960, S. $311 / 312$

38 Mommsen 1960, S. 313/314

39 Berthold, Lothar/Diehl, Ernst (Hrsg.): Revolutionäre deutsche Parteiprogramme. Vom kommunistischen Manifest zum Programm des Sozialismus. Berlin ${ }^{3} 1967$, S. 107-118

40 Mommsen 1960, S. 519-531

41 Rosenberg, Alfred (Hrsg.): Das Parteiprogramm. Wesen, Grundsätze und Ziele der NSDAP. München ${ }^{24} 1942$, S. 15-18

42 Mommsen 1960, S. 533-543

43 Mommsen 1960, S. 544/545

44 Mommsen 1960, S. 722/723

45 Berthold/Diehl 1967, S. 208-337

46 Hergt 1975, S. 231-241

47 Hergt 1975, S. 309-342 
- die Freiburger Thesen der Freien Demokratischen Partei (FDP), 197148;

- das Berliner Programm der Christlich-Demokratischen Union Deutschlands (CDU), ${ }^{2} 1971^{49}$;

- das Wahlprogramm der Sozialdemokratischen Partei Deutschlands (SPD), 197250;

- das Düsseldorfer Programm der Nationaldemokratischen Partei Deutschlands (NPD), 197351;

- das Grundsatzprogramm Freiheit, Solidarität, Gerechtigkeit der Christlich-Demokratischen Union Deutschlands (CDU), 197852;

- das Bundesprogramm Saarbrücker Programm der Grünen (GRÜNE), ${ }^{2} 1980^{53}$;

- das Grundsatzprogramm der Sozialdemokratischen Partei Deutschlands (SPD), 198954;

- das Grundsatzprogramm Freiheit in Verantwortung der Christlich Demokratischen Union Deutschlands (CDU), 199455;

- das Grundsatzprogramm Die Zukunft ist grün von BÜNDNIS 90/DIE GRÜNEN (B 90/GRÜNE), 200256;

- das Parteiprogramm der Feministischen Partei DIE FRAUEN, 200257;

- das Programm der Partei des Demokratischen Sozialismus (PDS), $2003^{58}$.

48 Hergt 1975, S. 249-273

49 Hintze, Peter: Die CDU-Parteiprogramme. Eine Dokumentation der Ziele und Aufgaben. Bonn 1995, S. 47-87

50 Hergt 1975, S. 87-121

$51 \quad$ Hergt 1975, S. 342-375

52 Hintze 1995, S. 123-167

53 BÜNDNIS 90/DIE GRÜNEN/GAL Münster. <http://www.gruenemuenster.de/modules.php?op=modload \&name=Downloads\&file=index\&req= getit\&lid=31> (26.08.2004)

54 SPD-Parteivorstand (Hrsg.): Grundsatzprogramm der Sozialdemokratischen Partei Deutschlands. Berlin 1998, S. 3-58

55 Hintze 1995, S. 367-439

56 BÜNDNIS 90/DIE GRÜNEN. <http://www.gruene-nrw.de/fileadmin /user_upload/files/Allgemein/Grundsatzprogramm-BV-2002.pdf > (26.08.2004)

57 Feministische Partei DIE FRAUEN. <http://www.feministischepartei.de /progra-d.htm> (03.09.2004)

58 PDS. <http://sozialisten.de/partei/grundsatzdokumente/programm /programm2003.pdf $>(15.09 .2004)$ 


\subsection{Themenspektrum und Ausblick}

Eine Untersuchung über politische Programme, deren Aussagen, Vokabular und Stil, ist gleichermaßen reizvoll wie schwierig. Reizvoll, weil das Stichwort Parteiprogramm gemeinhin spontane Assoziationen wie langweilig, geistlos, Neckermannkatalog u.ä. provoziert, und schwierig, weil der Fülle des dazu vorhandenen empirischen Materials der Mangel an systematischen Arbeiten und eindeutigen Kriterien zur Verarbeitung dieses Materials entspricht. ${ }^{59}$

Bevor das Godesberger Programm und die anderen Parteiprogramme nachfolgend beleuchtet werden, soll hier das weitere Vorgehen kurz skizziert werden.

\subsubsection{Methoden der Untersuchung und Aufbau der Arbeit}

Die oben zitierten drei Kategorien Aussagen, Vokabular und Stil sollen hinsichtlich der Parteiprogramme zur Beschreibung der sprachlichen Struktur analysiert werden; demnach ist die Untersuchung nach den Schwerpunkten der Pragmatik als sprachhandlungstheoretischem Ansatz und der einhergehenden Funktion der politischen Sprache, der Lexik und Semantik als Wortschatzcharakteristik sowie des Stils als linguistische und charakteristische Konzeption untergliedert.

Es geht um eine ganzheitliche Stilistik mit Einheiten wie Handlung (und ihren Voraussetzungen), Text (und seinen Funktionen), Thema (und seinen Bezügen). Es ist deshalb der Tendenz nach eine Stilistik ,von oben', vom Ganzen zu den Teilen verlaufend. Demgegenüber dominiert in der Stilistik noch immer der Blick ,von unten', wobei oft die Integrierung der Elemente zu einem Ganzen Schwierigkeiten bereitet. ${ }^{60}$

Dennoch soll in dieser Untersuchung die Betrachtung der Sprachhandlungen vor der stilistischen Beschreibung erfolgen. Die Unterkapitel der Semantik und Lexik mit der Erstellung des Wortschatz-Profils sowie die grammatikalische bzw. systemlinguistische Analyse werden dem Exkurs in die Stiltheorie unmittelbar folgen. Es soll daher der Versuch unternommen werden, die zuvor dargestellten theoretischen Aspekte der Stilistik in die jeweiligen Analysen zu integrieren.

Daher wird die Untersuchung mit den pragmatischen Aspekten der Textorganisation beginnen; als sprachhandlungstheoretischer Ansatz

59 Schönbohm, Wulf: „Funktion, Entstehung und Sprache von Parteiprogrammen." In: Aus Politik und Zeitgeschichte B 34-35. Hg. v. Bundeszentrale für politische Bildung. Bonn 1974, S. 17

60 Sandig, Barbara: Stilistik der deutschen Sprache. Berlin/New York 1986, S. 15 
sollen diese unter Punkt 2.1 analysiert werden. Anschließend werden die lexikalischen und systemlinguistischen Besonderheiten des Textes herausgearbeitet.

Im Zuge der pragmatischen Untersuchung sind der kommunikative und situative Kontext zu berücksichtigen sowie deren Auswirkung auf die Bedeutung einzelner Ausdrücke; folglich ist die Sprachverwendung als "angewandte Semantik"61 Gegenstand dieser Analysekategorie. In diesem Zusammenhang kann es auch interessant sein, die sich in den Texten manifestierenden politischen Absichten und Zielbestrebungen der Parteien darzustellen. In einem weiteren Analyseschritt sollen die zuvor gewonnen Erkenntnisse dann dazu beitragen, die Funktion des Textes herauszuarbeiten.

Zentrale Fragen in diesem Bereich stellen sich weiterhin nach den Argumentationsstrukturen, nach Behauptungen und möglichen Versprechungen. Welche Forderungen stellt die SPD in ihrem Grundsatzprogramm, wie werden diese formuliert, wie formulieren die NSDAP oder die CDU ihre Forderungen, und lassen sich möglicherweise Vermutungen treffen über die Wirkung, die solche Formulierungen auf den Empfänger haben können? Es wird folglich ausführlich auf die Rezeption von Parteiprogrammen einzugehen sein.

Die semantische und lexikalische Analyse der Parteiprogramme stellt den zweiten Untersuchungsbereich dar. Die Untersuchung beabsichtigt, mit Hilfe der Erstellung eines lexikalischen Profils des Godesberger Programms als Primärtext sowie ausgewählter Beispiele anderer Parteiprogramme Aufschluss über die Aspekte der sprachlichen Gestalt zu geben. Die Untersuchung in dieser Kategorie wird daher mit einer formalen Textbeschreibung sowie der Darstellung der Systemstellen, der dominanten Wortschatzsektoren, der Wortfamilien und Wortfelder beginnen und sich daran anschließend kleineren Analysekategorien zuwenden. So werden danach die im Godesberger Programm verwendeten Komposita, Wortschöpfungen und Substantivierungen unter dem Aspekt der Wortbildung zu untersuchen sein; die Frage nach den Erscheinungsformen, den Elementen und Strukturen komplexer Wörter wird sich hierbei stellen. Welche Ausdrücke werden (i) im Godesberger Programm und anderen Programmen verwendet und (ii) in welchen Kontexten kommt ein gegebener Ausdruck vor? Weiterhin werden Fremdwörter und mögliche idiomatisch oder metaphorisch verwendete Ausdrücke herausgestellt sowie solche, die als Schlüssel- oder Schlagwörter programmatisch verwendet $\mathrm{zu}$ sein scheinen.

61 Volmert, Johannes (Hrsg.): Grundkurs Sprachwissenschaft. Eine Einführung in die Sprachwissenschaft für Lehramtsstudiengänge. München ${ }^{31999, ~ S . ~} 171$ 
Nicht beabsichtigt wird, die für die zugrunde liegenden Texte charakteristischen grammatikalischen und systemlinguistischen Eigenschaften herauszustellen, obwohl sich hinsichtlich der Struktur von Sätzen, der Wortstellung und Satzarten sowie der Verwendung bestimmter Verben und charakteristischer Attribute ebenso zentrale Fragen stellen wie bezüglich der in den Programmen vorkommenden Wahlslogans bzw. Parolen, rhetorischen Fragen oder Appositionen.

Der Stil eines Textes ist maßgeblich geprägt durch dessen spezifische Eigenschaften. Diese wiederum machen die Textstruktur und den Stil erkennbar und wahrnehmbar. Zwar übt auch der Bereich der Pragmatik einen gewissen Einfluss auf den Stil eines Textes aus, bei der Untersuchung des Stils sollen jedoch die Bereiche der Lexik und Semantik (2.2.1) im Vordergrund stehen.

Direkte Zitate der Parteiprogramme sind eingerückt wiedergegeben und nummeriert, signifikante und wichtige Ausdrücke oder Textstellen innerhalb der jeweiligen Zitate sind durch Unterstreichung hervorgehoben. Zitierte Wörter und Sätze im laufenden Text sind in kursiver Schrift wiedergegeben. Die Zahl in Klammern hinter den jeweiligen Ausdrücken verdeutlicht die Häufigkeit der Erwähnungen im jeweiligen Parteiprogramm. Die Analyse wird versuchen, einen Einblick in die Theorie des jeweiligen Untersuchungsschwerpunkts darzubieten und mit ausgewählten exemplarischen Textstellen der Parteiprogramme zu unterfüttern.

Alle oben angeführten Themenschwerpunkte sollen sich in der Untersuchung immer wieder an der politischen Sprachverwendung orientieren. Daher decken die vorgestellten Punkte die jeweiligen Disziplinen Pragmatik, Lexik/Semantik oder die systemlinguistische Analyse nicht vollständig ab, sondern resultieren aus der Fragestellung nach der Rolle, die sie für die Textsorte Parteiprogramm spielen. Die zentralen Untersuchungspunkte sind aus der nachfolgend zitierten These abgeleitet:

Verlange vom Publikum das Minimum an geistiger Anstrengung. Deshalb bedarf es des starken Pathos in der Aussageweise, des Superlativistischen, der sprachlichen Verkürzung, der stereotypen Formeln, der Banal-, Trivial- und Leerformeln, der Klischees, der Schlagwörter, der Slogans usw. ${ }^{62}$

62 Straßner, Erich: Ideologie - SPRACHE - Politik. Grundfragen des Zusammenhangs. Tübingen 1987, S. 47 


\section{EXEMPLARISCHE ANALYSEN DER PARTEIPROGRAMME}

\subsection{Pragmatische Aspekte der Textorganisation}

Thema der Pragmatik ist das, was im Sprachgebrauch die Form und/oder die Interpretation sprachlicher Äußerungen regelhaft beeinflusst kraft der Tatsache, dass Sprache in einer Situation und zur Kommunikation, zum sprachlichen Handeln mit anderen, gebraucht wird. ${ }^{63}$

Die Pragmatik als Lehre vom Sprachhandeln bezieht sich folglich auf das Gesagte und Mitgeteilte unter der Fragestellung, wie sich das, was in einer Situation sprachlich realisiert wird, zu dem verhält, was mit dieser Äußerung über die Welt ausgesagt wird; es geht um „Fragen des Gebrauchs sprachlicher Ausdrücke zum Vollzug sprachlicher Handlungen." 64

Das oben angeführte Zitat verdeutlicht durch die Verwendung des Ausdrucks Gebrauch die in der Einleitung bereits verwendete Bezeichnung der Sprachverwendung (insbesondere der politischen) als Werkzeug. Diese Betrachtung wird insbesondere bei FINEGAN (1999) deutlich:

People use language principally as a tool to do things: ask questions, request favors, make comments, report news, give directions, offer greetings, and perform hundreds of other ordinary verbal actions in daily life. Through language, people actually do things: propose marriage, declare a mistrial, swear to tell the truth, fire an employee, invite someone to dinner, and so on. These speech acts are part of speech events such as conversations, lectures, student-teacher conferences, news broadcasts, marriage ceremonies, and courtroom trials. ${ }^{65}$

Unter dem Begriff der Pragmatik werden die verschiedenen Betrachtungsweisen der Situationsverbundenheit sprachlicher Äußerungen zusammengefasst; der Ausdruck pragmatisch ist daher im Sinne von sprachhandlungstheoretisch $\mathrm{zu}$ verstehen. Bei der Analyse eines Textes unter dem Aspekt der Pragmatik ist das Verhältnis sprachlicher Äußerungen $\mathrm{zu}$ ihrem situativen und kommunikativen Kontext folglich $\mathrm{zu}$ berücksichtigen:

63 Linke, Angelika/Nussbaumer, Markus/Portmann, Paul R.: Studienbuch Linguistik. Tübingen ${ }^{3} 1996$, S. 177

64 Gloning, Thomas: „Praktische Semantik und Linguistische Kommunikationsanalyse." In: Handbuch der Dialoganalyse. Hg. v. Gerd Fritz/Franz Hundsnurscher. Tübingen 1994, S. 114

65 Finegan, Edward: Language - Its Structure and Use. Fort Worth ${ }^{3} 1999$, S. 296 
Die illokutive Qualität einer Äußerung wird also nicht allein (und nicht unbedingt) festgelegt durch die Wahl der Wörter und grammatischen Formen, sondern vor allem durch gesellschaftliche Rollen, Verhaltenserwartungen (bzw. Verhaltenskonventionen) und die einer gesellschaftlichen Situation innewohnenden Sinnbezüge. ${ }^{66}$

Auf die oben zitierten illokutiven Komponenten der Sprachhandlungen, d.h. die illokutiven Akte als in den sprachlichen Äußerungen enthaltene Nebenbedeutungen, soll später ausführlicher eingegangen werden. Wichtig zu erwähnen ist in diesem Zusammenhang jedoch, dass die Wahl der Wörter (Lexik/Semantik) und der grammatischen Formen (Systemlinguistik) allein eine Analyse der politischen Sprachverwendung nicht abzudecken vermögen und die Pragmatik daher als grundlegender Untersuchungsschwerpunkt notwendig und gerechtfertigt ist. Nach KLEIN (1998) findet die politische Sprachverwendung auf drei sprachlichen Strukturebenen statt: „auf der Ebene der Wörter und festen Wortkombinationen, der Ebene der Sprechakte - meist in Form von Sätzen und Satzfolgen - sowie der Ebene der Makroformen Text, Dialog, Sendung und Veranstaltung. . ${ }^{67}$

\subsubsection{Zum Verhältnis von Sprache und Politik}

Nach GREIFFENHAGEN (1980) ist Sprache nicht nur

ein wichtiges Mittel des Politikers, sondern das Element, in dem sein Beruf sich vollzieht. Was er auch tut, auf welchem Felde er auch wirkt, stets arbeitet er mit dem geschriebenen, gelesenen, gehörten oder gesprochenen Wort: Er liest diplomatische Korrespondenz, Sitzungsprotokolle, Geheimdienstberichte, Zeitungskommentare, wissenschaftliche Gutachten, Akten aller Art. Er schreibt Briefe, diplomatische Noten, parlamentarische Reden. Er formuliert Depeschen, Wahlprogramme, Werbetexte. Er arbeitet Verträge aus. Seine Anweisungen, Korrekturen und Bemerkungen finden sprachlich unterschiedlichste Formen. Der Arbeitstag des Politikers besteht aus Beratungen, Sitzungen, öffentlichen Reden, Diktaten, Empfängen, Arbeitsessen, diplomatischen Adressen. Das Leben des Politikers ist reden, schreiben, lesen: Umgang mit dem Wort. Dabei bewegt sich der Politiker in den verschiedensten Sprachfeldern, Sprachebenen und Sprachstilen. ${ }^{68}$

66 Bachem 1979, S. 19

67 Klein, Josef: „Politische Kommunikation als Sprachstrategie.“ In: Politische Kommunikation in der demokratischen Gesellschaft. Ein Handbuch mit Lexikonteil. Hg. v. Otfried Jarren/Ulrich Sarcinelli/Ulrich Saxer. Wiesbaden 1998, S. 376

68 Greiffenhagen, Martin: Kampf um Wörter? Politische Begriffe im Meinungsstreit. München/Wien 1980, S. 9 
Folglich verbringen Politiker einen großen Teil ihrer Zeit im aktiven Umgang mit Sprache. Sie halten Ansprachen, debattieren, diskutieren, geben Interviews und Pressemitteilungen, halten Lobes-, Dankes- und Parlamentsreden und geben Regierungserklärungen ab. Zudem feilen sie an Formulierungen in Gesetzestexten, Parteiprogrammen oder Koalitionsverträgen;

ob wir dabei aber an politisches Handeln, ob wir an politische Theorie oder an politische Praxis, an politische Prozesse, Institutionen oder Herrschaft denken, immer ist Politik unlösbar an Sprache gebunden. ${ }^{69}$

Ist Politik nun (i) mit Kommunikationsprozessen verbunden, oder ist Politik (ii) unabdingbar an Kommunikationsprozesse gebunden? Die sich aufdrängende Frage nach dem Anteil der sprachlichen Handlungen am gesamten politischen Handeln wird - je nach Blickwinkel - unterschiedlich beantwortet.

„Am einen Extrempol liegt die Auffassung, daß alles politische Handeln sprachliches Handeln ist. " 70 Dass Sprechen keinen Gegensatz zum Handeln darstellt, kann auch mit WEBER (1920) gezeigt werden, denn ,,'Handeln' soll [...] ein menschliches Verhalten (einerlei ob äußeres oder innerliches Tun, Unterlassen oder Dulden) heißen, wenn und insofern als der oder die Handelnden mit ihm einen subjektiven Sinn verbinden. ${ }^{\text {71 }}$

Die andere Position sieht dagegen sprachliches Handeln als Spezialform politischen Handelns; diese Position betont folglich die außersprachlichen Faktoren der Politik und räumt der politischen Sprachverwendung zwar eine wichtige, aber nicht eine entscheidende Rolle ein: „Die Sprache der Politik ist zwar keineswegs der entscheidende Aspekt der Politik, aber sie ist eine wichtige Komponente im politischen Geschehen. "${ }^{72}$ Nach dieser Auffassung kann man die politische Sprachverwendung betrachten als einen

in sich differenzierten Großbereich der Kommunikation, in dem über Angelegenheiten öffentlichen Interesses gehandelt wird, in dem Meinungen gefaßt werden und Prozesse ablaufen, die der Herstellung

69 Grünert, Horst: „Politische Geschichte und Sprachgeschichte. Überlegungen zum Zusammenhang von Politik und Sprachgebrauch in Geschichte und Gegenwart." In: Sprache und Literatur in Wissenschaft und Unterricht (Band 52). München/Paderborn 1983, S. 43

70 Holly, Werner: Politikersprache. Inszenierungen und Rollenkonflikte im informellen Sprachhandeln eines Bundestagsabgeordneten. Berlin/New York 1990, S. 4

71 Weber, Max: „Soziologische Grundbegriffe.“ In: Max Weber. Schriften 18941922. Hg. v. Dirk Kaesler. München 2002, S. 653

72 Klaus, Georg: Sprache der Politik. Berlin (Ost) 1971, S. 9 
und Durchsetzung verbindlicher oder auch umstrittener gesellschaftlicher Entscheidungen dienen. ${ }^{73}$

Während die erste Position, d.h. „Sprache ist nicht nur irgendein Instrument der Politik, sondern überhaupt erst die Bedingung ihrer Möglichkeit ${ }^{\star 74}$, von den sprachhandlungsorientierten Ansätzen der Linguistik bevorzugt wird, wird letztere in der Regel in politikwissenschaftlichen Untersuchungen vertreten. Dabei sollte die Problematik einer mangelnden Distanz zum Untersuchungsgegenstand aufgrund möglicher Politisierung stets berücksichtigt werden:

Nicht selten ist an Arbeiten zur politischen Sprache eine mangelnde Distanz zum Untersuchungsgegenstand zu konstatieren. In selber politisierter Sprache wird über die politische Sprache gesprochen und geschrieben. ${ }^{75}$

Zwar sollen in dieser Untersuchung die außersprachlichen Motive nicht völlig vernachlässigt werden (eine scharfe Trennung ist ohnehin kaum möglich), der Schwerpunkt soll jedoch auf der ersten Position beruhen und geht daher mit der bereits dargelegten Betrachtung der Sprachverwendung als Werkzeug einher.

Die Sprache der Politik kann als ein Medium betrachtet werden, dessen Bedeutung über ihre Verständigungsfunktion weit hinausgeht. Es lassen sich nun zwei Fragestellungen formulieren: Erstens, wie kann Sprache als Werkzeug für die politische Kommunikation verwendet werden und zweitens, wie schlägt sich die Verwendung von Sprache konkret in ihrer Verwendungsweise nieder. So schreibt GRÜNERT (1983): „Politik wird durch (mit) Sprache entworfen, vorbereitet, ausgelöst, von Sprache begleitet, beeinflußt, gesteuert, geregelt, durch Sprache beschrieben, erläutert, motiviert, gerechtfertigt, verantwortet, kontrolliert, kritisiert, beund verurteilt." ${ }^{\circ 6}$

Der Ausdruckskomplex Sprache und Politik beinhaltet daher sowohl die Betrachtungsweise Sprache als Gegenstand der Politik als auch die Betrachtungsweise Sprache der Politik. Die oben beschriebenen Betrachtungsweisen stimmen überein, denn

in beiden Betrachtungsweisen verfolgen Menschengruppen mit verwaltungsmäßigen und sprachlichen Mitteln das Ziel, Verhaltenswei-

73 Strauß, Gerhard/Haß, Ulrike/Harras, Gisela: Brisante Wörter - von Agitation bis Zeitgeist. Ein Lexikon zum öffentlichen Sprachgebrauch. Berlin/New York 1989, S. 29

74 Girnth, Heiko: Sprache und Sprachverwendung in der Politik. Eine Einführung in die linguistische Analyse öffentlich-politischer Kommunikation. Tübingen 2002, S. 1

75 Klein 1996b, S. 84

76 Grünert 1983, S. 43 
sen von Menschen zu beeinflussen, praktiziertes Verhalten zu bestärken oder es durch neue Verhaltensmaßstäbe zu verändern und abzulösen. ${ }^{77}$

HERINGER (1990) schlägt den Terminus Politik in Sprache anstelle Sprache in der Politik vor: ",,Politik in Sprache' soll darauf hinweisen, daß Politik sich in Sprache vollzieht, daß politische Tätigkeit sprachliche Tätigkeit ist. ${ }^{\text {"78 }}$

Unabhängig von der begrifflichen Unklarheit der Ausdrücke Sprache der Politik, Sprache und Politik, politische Sprache, Politik der Sprache, der institutionsinternen Sprache in der Politik oder die institutionsexternen, d.h. „unidirektional über die Medien an die Bürger gerichtete Politikersprache ${ }^{\prime 79}$ geht es wesentlich darum, die öffentliche Kommunikation über politische Fragen und Sachverhalte und die mit deren wissenschaftlicher Analyse beschäftigte Forschungsdisziplin (Politolinguistik) zu bezeichnen:

Als unterschiedliche situations- und adressatenspezifische Sprechweisen politischer Funktionsträger ließen sich beide Bereiche [Sprache in der Politik und Politikersprache] unter dem gemeinsamen Dach einer Politiksprache zusammenfassen, der das private bis halböffentliche Sprechen über Politik gegenübersteht. Zwischen der Politiksprache einerseits und dem Sprechen über Politik andererseits vermittelt die Politische Mediensprache als Kommunikationsform des politischen Journalismus..$^{80}$

Für alle Arten öffentlichen oder privaten Sprechens über politische Fragen sowohl innerhalb als auch außerhalb des institutionellen Rahmens der Politik kann der Ausdruck politische Sprache als Oberbegriff gelten; aus diesen begrifflichen Differenzierungen ergibt sich somit das folgende Schaubild auf der nächsten Seite:

77 Bergsdorf 1978, S. 49

78 Heringer, Hans Jürgen: ,Ich gebe Ihnen mein Ehrenwort.' Politik - Sprache Moral. München 1990, S. 9

79 Burkhardt 1996, S. 80

80 Burkhardt 1996, S. 80/81 


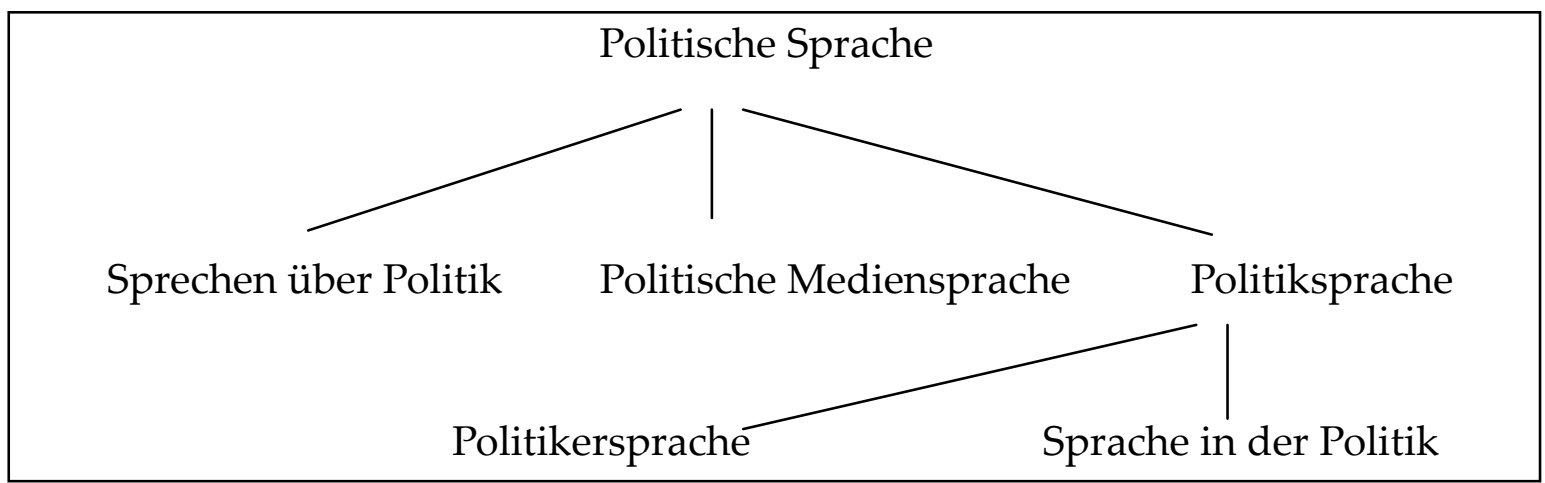

Abbildung 1: (vgl. BURKHARDT 1996, S. 81)

Mit Hilfe der Sprache soll somit versucht werden, das Verhalten von Menschen den kulturellen und politischen Zielvorstellungen anzupassen. Politische Kommunikation ist folglich primär darauf ausgerichtet, zu überzeugen und für sich einzunehmen; dies ist ihr bestimmtes kommunikatives Ziel. Eine solche Absicht manifestiert sich in der Wahl einer Äußerung hinsichtlich ihrer Bedeutung oder der Wahl eines Inhalts, aber auch in der Berücksichtigung der Adressatengruppe. Diese pragmatische Selektion ist somit außersprachlich motiviert, da es einer Partei beispielsweise darum geht, die Regierung für eine weitere Legislaturperiode zu stellen. Die Wahl der sprachlichen Handlungsmuster resultiert daher aus diesen Motiven: „Der Handlungszweck der sprachlichen Interaktion beruht auf den Handlungszielen der Interaktionspartner und hängt eng mit ihnen zusammen." ${ }^{\text {81 }}$

Die Wahl orientiert sich jedoch ebenso an der Aussage der sprachlichen Handlung: „pragmatische Wahl zieht also den Entscheid nach sich, was eine Person mit ihrer linguistischen Mitteilung aussagen will." ${ }^{82}$ Im Hinblick auf das Parteiprogramm sollten deskriptive und präskriptive Aussagen nicht verwischen; es sollte daher erkennbar sein, welche Aussagen den Willen der Partei ausdrücken und welche Aussagen als Beschreibung einer Situation zu werten sind.

Auch wenn man der Sprache innerhalb der Politik eine bedeutende Funktion zubilligt, sollte man nicht vergessen, dass in der Politik neben der verbalen auch nonverbale Kommunikation stattfindet, insbesondere im Rahmen massenmedialer Ereignisse; in diesem Bereich spielt beispielsweise die Körpersprache (Gestik und Mimik) eine bedeutende Rolle sowie allgemeine charismatische Eigenschaften der in der Öffentlichkeit stehenden Politiker. Weiterhin ist die Arbeit mit politischen

81 Hundsnurscher, Franz: „Dialog-Typologie.“ In: Handbuch der Dialoganalyse. Hg. v. Gerd Fritz/Franz Hundsnurscher. Tübingen 1994, S. 224

82 Enkvist, Nils Erik: „Versuche zu einer Bestimmung des Sprachstils: Ein Essay in angewandter Sprachwissenschaft." In: Linguistik und Stil. Hg. v. John Spencer. Heidelberg 1972, S. 32 
Symbolen (z.B. Wahlplakate) von suggestiver Wirksamkeit. Daher wird nicht nur in autoritären Staaten, sondern auch in demokratischen Regierungen mit politischer Symbolik gearbeitet; darunter fallen beispielsweise "Nationalsymbole wie Nationalfarben, flaggen, oder -hymnen, Herrschaftssymbole wie der Adler, Freiheitssymbole wie die Fackel (etwa in der Freiheitsstatue der USA) oder Einigungssymbole wie die Weltkugel (als politisches Symbol der UN). ${ }^{183}$ Folglich sind sprachliches Handeln und politisches Handeln trotz der exponierten Rolle der Sprachverwendung als hauptsächliches Medium der Politik nicht identisch.

\subsubsection{Sprache und Ideologie}

Der heutige moderne Ideologie-Begriff reicht in das 18. Jahrhundert zurück und wurde im geschichtlichen Lauf fortwährend unterschiedlich verwendet. Die Kennzeichnung des Begriffs Ideologie als Weltanschauung oder Gedankengebilde und insbesondere als Grundüberzeugung bildet die Grundlage für dessen Verwendung in dieser Untersuchung. Eine pluralistische Gesellschaft ermöglicht die Herausbildung und Etablierung unterschiedlicher Grundüberzeugungen; die Parteienlandschaft mit den verschiedenen Ideologien verdeutlicht diese These. Während in politischen und politiktheoretischen Debatten der Ideologie-Begriff in der Regel stigmatisierend für den politischen Gegner verwendet wird, soll hier eine wertneutrale Verwendung des Begriffs etabliert werden. Unter Ideologie sollen "die einer bestimmten gesellschaftlichen Gruppierung oder einer bestimmten Gesellschaftsordnung zugrundeliegenden Wertvorstellungen und Denkmuster" ${ }^{\prime 84}$ verstanden werden. STRABNER (1987) fasst den Begriff der Ideologie

als das Sinnsystem, das seine normative Legitimation von Herrschaftsformrn zwar als in rationalem Diskurs begründet ausgibt, denn anders wäre sie für kein vernunftsfähiges Wesen akzeptierbar, gleichzeitig aber jeden tatsächlichen rationalen Diskurs, der sie infragestellen oder aufheben würde, systematisch verhindert. ${ }^{85}$

In Sinne der ersten Definition werden alle den untersuchten Parteiprogrammen zugrunde liegenden Theorien wie Liberalismus, Sozialismus, Konservatismus, Nationalismus, Kommunismus und Nationalsozialismus in dieser Untersuchung als Ideologien betrachtet, aus denen sich wiederum „bestimmte Folgerungen für die Gestaltung einzelner Teilbereiche von Staat, Wirtschaft und Gesellschaft" ${ }^{\prime \prime 6}$ ableiten lassen - dies gilt ebenso für die Verwendung der Sprache. Die Verwendung des Ideologie-Begriffs ist

\footnotetext{
83 Girnth 2002, S. 2

84 Girnth 2002, S. 2

85 Straßner 1987, S. 6

86 Politische Ideologien 1990, S. 1
} 
jedoch nicht unproblematisch, insbesondere wenn sich die Parteien selbst gegen diesen Begriff aussprechen:

(a) Uns eint, uns verbindet ein Kreis von Grundwerten, nicht eine Ideologie. (B 90/GRÜNE 2002, S. 9)

Bei der Betrachtung der ideologischen Aspekte in der politischen Sprachverwendung ist grundsätzlich festzuhalten, dass Sprache als Ganzes klassenindifferent ist; jedoch finden Klassen- bzw. Schichtinteressen ihren Ausdruck in den spezifischen Ideologien der Klassen, die sich wiederum in der jeweiligen Sprachverwendung widerspiegeln. Politischem Handeln und Sprechen liegen unterschiedliche Interessen zugrunde, die ihrerseits die Handlungs- und Sprechhandlungsintentionen determinieren. Die politische Sprachverwendung ist notwendigerweise parteilich, da die politische Kommunikation von Interessenkonflikten sowie von unterschiedlichen Meinungen und Weltanschauungen geprägt ist und folglich die lexikalischen Elemente in ideologisch gegensätzlichen Verwendungen gebraucht werden. Die politische Sprachverwendung bedient sich daher ideologisch wertender Sprachformen.

Will man das Verhältnis von Ideologie und Politik charakterisieren, dann kann man konstatieren, dass „politische Wirklichkeit immer ideologische Wirklichkeit ist. “87 Und wenn sich, wie oben gezeigt wurde, politisches Handeln in Sprache vollzieht, dann ist Sprache als Gesellschaftsprodukt auch der Ort, in dem sich Ideologien manifestieren. Da die Ideologie in politischen Auseinandersetzungen mit der Herabsetzung oder Diffamierung des Gegners einhergeht, wird dieser Themenkomplex erneut in den nachfolgenden Kapiteln Gegnerbezug und Erzeugung von Sichtweisen aufgegriffen, sowie weiterhin bei der Betrachtung der Schlagwörter und Metaphern.

Die Betrachtung der politischen Sprachverwendung unter dem Aspekt der Ideologie soll insbesondere bei der Wortschatzanalyse in dieser Untersuchung berücksichtigt werden, da sich die oben erwähnte Manifestierung besonders in der Wahl der Ausdrücke zeigt, die einen Sachverhalt beschreiben sollen: „Bei der Herausbildung einer Ideologie entsteht ein eigener Fachwortschatz, der ideologische Wortschatz, das heißt, das ,System der gesellschaftlichen (politischen, philosophischen, religiösen, künstlerischen usw.) Ideen [...]' findet seinen sprachlichen Ausdruck in einem terminologischen System." ${ }^{\text {88 }}$ Elemente eines ideologischen Wortschatzes bilden als Begriffe bestimmte Sachverhalte ab; die Begriffe sind danach ideologiegebunden, d.h. ihre Bedeutung als Termini ergibt sich

87 Strauß/Haß/Harras 1989, S. 28

88 Schmidt, Wilhelm: "Zur Ideologiegebundenheit der politischen Lexik." In: Zeitschrift für Phonetik, Sprachwissenschaft und Kommunikationsforschung (Band 22). Berlin 1969, S. 255 
erst in Zusammenhang mit dem terminologischen System der jeweiligen Ideologie und verliert sich außerhalb dieses Kontextes.

Linguistisch interessant ist [...] vor allem die Frage nach dem Zusammenhang zwischen Ideologien und typischen Formen ihrer Versprachlichung. Ideologischer Sprachgebrauch muss immer im Kontext der konkreten historisch-politischen Situation bewertet werden. ${ }^{89}$

Bei der ideologiegebundenen Sprache entwickelt sich allerdings nicht nur ein bestimmter ideologiegeprägter Wortschatz - es bildet sich vor allem ein ideologischer Jargon heraus, dem sich durch folgende Merkmale zuschreiben lassen: „Reden in tautologisch sich selbst bestätigenden Sätzen, Verschwinden des Sinnes aus funktioniblen syntaktischen Einheiten, Verlust gedanklicher Prägnanz, Auszehrung der Wörter bis zum Verbleib bloßer Worthülsen, Verwischen logischer Bezüge und Zerfaserung der Syntax. " 90 Die politische Sprache läuft somit Gefahr, aufgrund einer möglichen Funktionalisierung zur Disposition beliebiger Irrationalisierung gestellt zu werden; die Parteiprogramme sollen nachfolgend auf die oben zitierten Merkmale untersucht werden.

\subsubsection{Sprache und gesellschaftliche Wirklichkeit}

Der wirklichkeitskonstituierende Charakter von Sprache wird nicht nur an Abstrakta wie beispielsweise Freiheit oder Gerechtigkeit deutlich, „,die allein durch ideologisch gebrochene Begriffsbildung kommunikativ verfügbar werden. “91 Welchen Einfluss hat Sprache nun auf gesellschaftliche Veränderungen? Auf diese Frage gibt es eine sprachidealistische und eine sprachmaterialistische Antwort. Da sprachliche Strukturen die Wahrnehmung und die einhergehenden Handlungen beeinflussen, beschreibt die sprachidealistische Auffassung diesen Einfluss der sprachlichen Handlungsmuster auf die Sicht des Menschen; die gesellschaftliche Wirklichkeit wird folglich durch die Sprache determiniert. BURKHARDT (1988) geht davon aus, dass durch Sprache Handlungen vollzogen werden und durch Äußerungen Fakten, soziale Tatsachen, geschaffen werden:

Das ändert jedoch nichts an der Tatsache, daß durch Äußerungen soziale Tatsachen geschaffen werden, nicht zuletzt in der Sprache der Politik, insbesondere in den politischen Institutionen, wo nicht nur (rhetorische) Fragen gestellt, (falsche) Versprechungen gemacht, (unwahre) Behauptungen aufgestellt oder (ausweichende) Antworten gegeben, sondern z.B. auch Gesetze verabschiedet, Programme beschlos-

\footnotetext{
89 Girnth 2002, S. 3

90 Straßner 1987, S. 24

91 Girnth 2002, S. 5
} 
sen, Drohungen und Ultimaten ausgesprochen, Kriege erklärt und die Wähler zu Wahlen aufgerufen werden. ${ }^{92}$

Dagegen steht die sprachmaterialistische Antwort mit ihrer Auffassung, dass es allein "die natürlichen und gesellschaftlichen Lebensbedingungen der Menschen [...] sind, die sein Bewußtsein und damit sein Sprechen und Handeln bestimmen. ${ }^{\prime \prime 3}$ Die Parteiprogramme werden beiden Auffassungen gerecht; sie spiegeln einerseits die gesellschaftlichen Bedingungen, d.h. die zeitgenössische Lebenswirklichkeit, mit all ihrer Widersprüchlichkeit, da die Parteien beabsichtigen, gegenwärtige Missstände zu beseitigen, andererseits soll die Wahrnehmung und damit das Handeln durch die Rezeption des Parteiprogramms in bestimmte Richtungen beeinflusst werden. Bei der politischen Sprachbetrachtung ist daher eine Position angemessen,

die zwischen der idealistischen und der materialistischen Auffassung vermittelt. Sprache und gesellschaftliche Wirklichkeit bedingen sich gegenseitig. Die gesellschaftliche Wirklichkeit manifestiert sich in Sprache, gleichzeitig wird sie aber überhaupt erst durch Sprache ermöglicht und hergestellt. ${ }^{94}$

\subsection{Parteiprogramme - Wirklichkeitsabbildung und Fachsprachlichkeit}

Im vorangegangenen Kapitel wurde das Parteiprogramm als wirklichkeitsabbildendes Sprachgebilde dargestellt; für diese Annahme spricht, dass ein Parteiprogramm die gegenwärtige gesellschaftliche Situation abbildet. Dieser Auffassung widerspricht allerdings die denkbare Eigenschaft der Fachsprachlichkeit eines solchen politischen Textes. Auch STRAßNER (1987) spricht sich gegen die Wirklichkeitsabbildung von Sprache aus:

Sprache bildet Wirklichkeit nicht ab. Sie ist vielmehr ein Handlungsleitsystem, das gewisse stets und notwendig perspektivische Deutungen der Erfahrung und ihrer Transformation in kognitiven Operationen reguliert. Ein Wort gibt so nicht etwa das Bezeichnete wieder, sondern weist auf die Bedeutung hin, die dem bezeichneten zugemessen wird. ${ }^{95}$

92 Burkhardt, Armin: „Sprache in der Politik. Linguistische Begriffe und Methoden.“ In: Englisch-Amerikanische Studien 10 (Heft 3, 4). Münster 1988, S. 339

93 Jäger, Siegfried: „Gesellschaftsveränderungen über Sprache.“ In: Soziolinguistik. Ein internationales Handbuch zur Wissenschaft von Sprache und Gesellschaft. Zweiter Halbband. Hg. v. Ulrich Ammon/Norbert Dittmar/Klaus J. Mattheier. Berlin/New York 1988, S. 1794

94 Girnth 2002, S. 6

95 Straßner 1987, S. 17 
Wie bereits im Vorwort erwähnt ist die Sprache der Politik weniger als Sondersprache oder reine Fachsprache zu verstehen, sie weist allerdings charakteristische Elemente einer Fachsprache auf (Teile des Wortschatzes und Formen der sprachlichen Kommunikation). Da die politische Sprache aber verschiedene Sprachstile und ideologische "Politolekte" ${ }^{\prime 96}$ mit jeweils unterschiedlicher Zielsetzung in sich vereint, ist die politische Sprache vielmehr als eine Varietät, als eine Zwischenschicht oder "ein Gemisch aus verschiedenen Fachsprachen und der Standardsprache ${ }^{\prime \prime 97}$ anzusehen. Problematisch ist die Abgrenzung der politischen Sprache von der Alltagssprache insbesondere aufgrund der Möglichkeit, nahezu jede gesellschaftliche Debatte zu einer politischen werden zu lassen. In einem solchen Fall bedient sich die Sprache der Politik bei Bedarf aus deren jeweiligen Fachsprachen und Expertenwissen. Diese Problematik soll an späterer Stelle erneut aufgegriffen und vertieft diskutiert werden, wenn das politische Fachlexikon analysiert wird.

Dennoch stellen Parteiprogramme Texte in der politischen Sprachverwendung dar, die durch bestimmte Merkmale geprägt sind, die auf eine Fachsprachlichkeit schließen lassen, denn insbesondere das Grundsatzprogramm dient weniger der Werbung und ist weniger an die Öffentlichkeit gerichtet (das ist Aufgabe des Wahlprogramms), sondern es dient vielmehr der Kommunikation zwischen Fachleuten; aus dieser geht es schließlich auch hervor. Daher fehlt den Grundsatzprogrammen der Parteien „die primäre Ausrichtung auf Nicht-Fachkundige."

Ein weiteres Merkmal der Fachsprachlichkeit ist das Vorhandensein eines Codes, „in dem fachspezifische Sachkenntnisse auf eine Weise encodiert werden, die in vollem Umfang nur Fachleuten verständlich ist." ${ }^{\prime 99}$ Der Ausdruck des Codes beschreibt den Gebrauch einer eigenen fachsprachlichen Lexik und Textsemantik, die für die Textgattung des Parteiprogramms charakteristisch sind:

Parteiprogramme, vor allem Grundsatzprogramme, zeichnen sich durch folgende Merkmale aus: Katalogstil, Bekenntnisse zu Selbstverständlichkeiten, Kompromiß-Formulierungen, kalkulierte Ambiva-

96 Kilian, Jörg: „Sprache in der Politik. Ein einführender Überblick.“ In: Praxis Deutsch (Band 21). Seelze 1994, S. 4

97 Kilian 1994, S. 4

98 Klein, Josef: „Insider-Lesarten. Einige Regeln zur latenten Fachkommunikation in Parteiprogrammen." In: Sprachstrategien und Dialogblockaden. Linguistische und politikwissenschaftliche Studien zur politischen Kommunikation. Hg. v. Josef Klein/Hajo Dieckmannshenke. Berlin/New York 1996a, S. 202

Klein 1996a, S. 201 
lenz, impliziten Gegnerbezug und ostentative Nicht-Erwähnungen.

Sie haben sämtlich fachkommunikative Bedeutung [...]. ${ }^{100}$

Die oben zitierten Merkmale sind Bestandteile des sprachlichen Codes eines Parteiprogramms, ohne dessen Kenntnis die jeweiligen Inhalte anders als vom Verfasser beabsichtigt wahrgenommen werden; daher soll an späterer Stelle ausführlich auf die genannten Merkmale eingegangen werden. Die Analyse der Parteiprogramme deckt jedoch nur einen Teilbereich der politischen Sprachverwendung ab; als Einstieg in die Analyse soll nachfolgend das Verhältnis zwischen Sprache und Politik allgemein beleuchtet werden.

\subsubsection{Funktionen von Sprache}

Bevor im Folgenden speziell der politische Sprachgebrauch untersucht wird, stellt sich die Frage nach der Funktion von Sprache allgemein. Eine erste spontane Antwort auf diese Frage könnte lauten: Sprache ist ein/das Medium, über das Menschen ihre Meinungen und Ideen, aber auch bestimmte Sachverhalte und Informationen miteinander austauschen. Jedoch lassen sich der Sprache noch andere wichtige Aufgaben zuschreiben.

BÜHLERS (1999) Organon-Modell101 geht auf das Jahr 1934 zurück; dem Modell folgend lassen sich die oben erwähnten Aufgaben nach einer dreifachen Funktion in Darstellung, Ausdruck und Appell untergliedern, wonach (i) ein Sprecher mit Hilfe der Sprache einen Sachverhalt beschreibt und darstellt, was dieser seinem Kommunikationspartner mitteilen will, (ii) der Sprecher seine emotionalen Bewegungen oder $\mathrm{Zu}$ stände auszudrücken beabsichtigt und Werteinschätzungen oder Befindlichkeiten kundtut sowie (iii) der Sprecher an seinen Gesprächspartner appelliert mit der Absicht, dessen Denken und Verhalten in seinem Sinn zu bestärken oder zu verändern, jedenfalls den Empfänger in einer bestimmten Weise zu beeinflussen. Zwar lassen sich sprachliche Äußerungen nicht eindeutig diesen Kategorien zuordnen. Eine Mitteilung als sprachliche Handlung wird jedoch die Ausdrucksseite in den Vordergrund rücken; die Absicht eines Sprechers, in erster Linie den Empfänger einer sprachlichen Kommunikation anzusprechen, bezieht sich auf die Appellfunktion und der Akzent wird auf der Darstellungsfunktion liegen, wenn die Mitteilung über Sachverhalte im Vordergrund der sprachlichen Kommunikation steht.

100 Klein 1996a, S. 203

101 Bühler, Karl: Sprachtheorie. Die Darstellungsfunktion der Sprache. Stuttgart ${ }^{3} 1999$, S. $28 \mathrm{ff}$. 
Analog zu BÜHLER (1999) teilt KLAUS (1971) auch den sprachlichen Zeichen drei Haupteigenschaften zu. Sämtliche sprachliche Zeichen sind designativ (beschreibend), appraisiv (bewertend) und preskriptiv (auffordernd). Somit ergibt sich das folgende Schaubild:

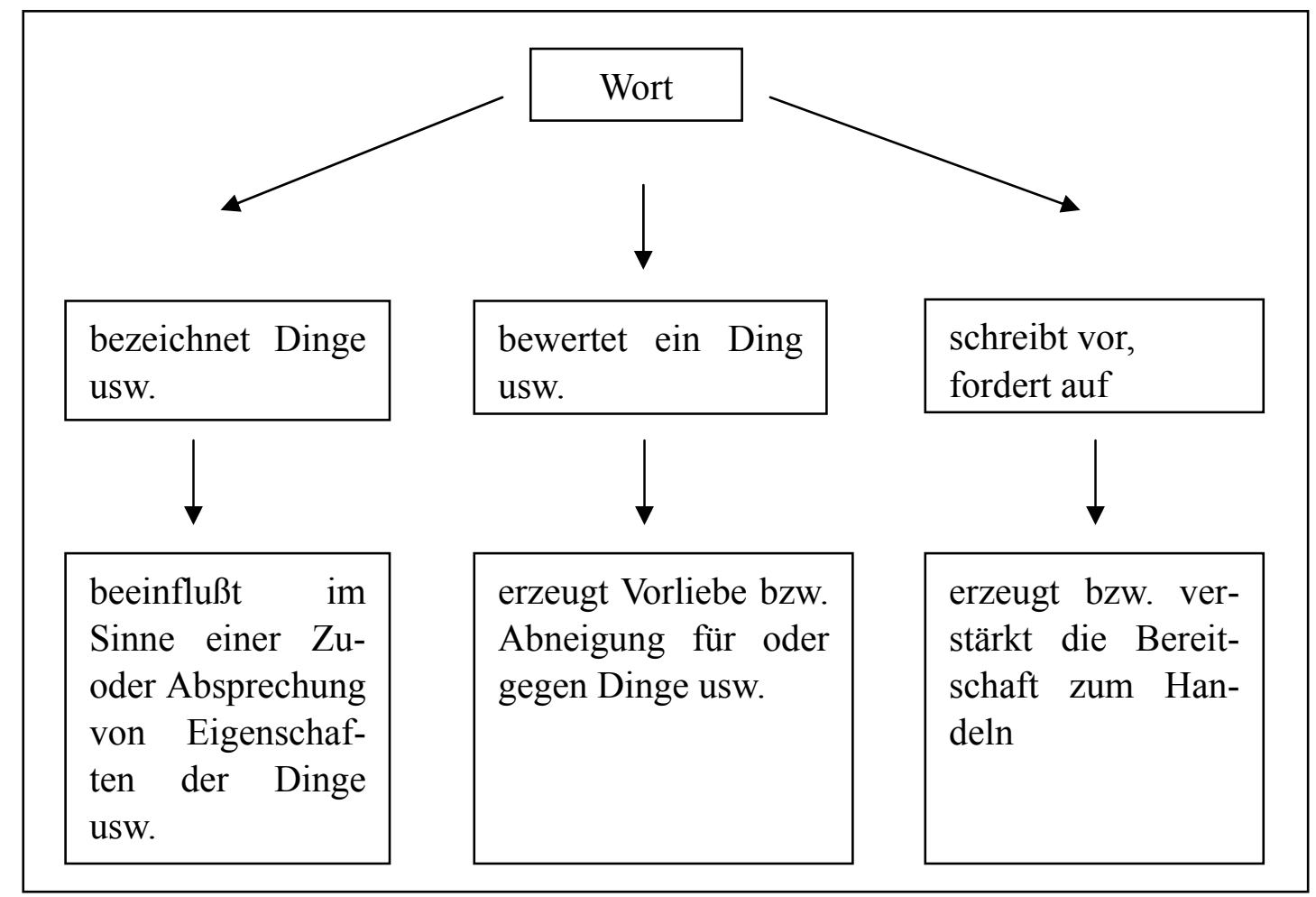

Abbildung 2: (vgl. KLAUS 1971, Abb. 2, S. 25)

Für das Adjektiv demokratisch ergeben sich somit drei Möglichkeiten der Verwendung bzw. drei Haupteigenschaften: „wissenschaftliche Bezeichnung eines bestimmten Gesellschaftszustandes (designativ), positive bzw. negative Bewertung eines bestimmten Zustandes (appraisiv), Aufforderung, diesen Zustand zu unterstützen bzw. zu bekämpfen (preskritiv). ${ }^{\prime 102}$

Die Definition zur Sprache der Politik in den vorangegangenen Kapiteln wurde nun um die zusätzliche Funktion, d.h. die Komponente der bestimmten Absicht, erweitert.

\subsubsection{Funktionen der politischen Sprachverwendung}

Die Absicht der politischen Sprachverwendung ist Ausdruck des Machtstrebens der Parteien. Eine der populärsten Definitionen des Machtanspruchs der Politik liefert WEBER bereits im Jahre 1919:

,Politik' würde für uns also heißen: Streben nach Machtanteil oder nach Beeinflussung der Machtverteilung, sei es zwischen Staaten, sei

102 Klaus 1971, S. 23 
es innerhalb eines Staates zwischen den Menschengruppen, die er umschließt. ${ }^{103}$

Warum das Zitat von WeBer (1919) an dieser Stelle? Folgt man dessen Definition von Politik als Streben nach Macht und berücksichtigt man weiterhin die in den vorangegangenen Kapiteln diskutierte Auffassung, dass politisches Handeln zu einem Großteil sprachliches Handeln bedeutet, dann stellt sich nun die Frage, wie dieser Machtanspruch und das politische Handeln durch die Sprache verkörpert werden, d.h. auf welche Weise sich das Streben nach Macht in der politischen Sprachverwendung niederschlägt. Nach KLEIN (1998) findet das politische Machstreben seine Realisierung in bestimmten Sprachstrategien:

Unter den Bedingungen politischer Konkurrenz ist der Erfolg politischer Kommunikation nicht zuletzt davon abhängig, wer über die leistungsfähigeren sprachlichen Ressourcen verfügt; daraus resultieren Sprachstrategien mit dem Zweck, die eigenen sprachlichen Ressourcen zu stärken und die der Konkurrenz zu schwächen. ${ }^{104}$

Die Sprachstrategien lassen sich untergliedern; so umfasst der Terminus Konkurrenzstrategie die "Stärkung eigener und [...] Beeinträchtigung gegnerischer Sprachressourcen“, die Kaschierstrategie dient dazu, "Verstöße gegen die Präferenzen relevanter Adressatengruppen und/oder gegen die kommunikationsethischen Normen der Informativität, der Wahrheit, der Relevanz und der Klarheit vor den Adressaten möglichst zu verbergen" und die Basisstrategie beschreibt die Strategie der "Orientierung an den Präferenzen relevanter Adressatengruppen, der Aufwertung eigener und/oder der Abwertung konkurrierender Positionen und der Abstimmung auf die massenmedialen Begebenheiten. "105

Weiterhin stellt sich die Frage, wie die Funktionen der politischen Sprachverwendung benannt und wie sie sich untergliedern lassen könnten:

Sprache gewinnt im politischen Zusammenhang instrumentelle Funktion: Als Mittel zum Machtgewinn, zur Machtausübung, zur Machtsicherung und zur Machtkontrolle. Über diese Akzentuierung der instrumentellen Funktion der Sprache darf aber, daran sei erinnert, nicht übersehen werden, daß Sprache im politischen Kontext auch eine konstitutive Funktion hat, daß nämlich schon die Intentionen poli-

\footnotetext{
103 Weber, Max: „Politik als Beruf.“ In: Politisches Denken im 20. Jahrhundert. Ein Lesebuch. Hg. v. Herfried Münkler. München 21997, S. 23

104 Klein 1998, S. 376

105 Klein 1998, S. 376
} 
tisch Handelnder durch Sprechakte konstituiert und stabilisiert sind. ${ }^{106}$

Handlungsziele werden folglich durch bestimmte kommunikative Verfahren erreicht. Die bereits im Zusammenhang mit den Sprachspielen genannten kommunikativen Verfahren sind als Problemlösungsverfahren auf der Ebene der Sprachverwendung zu betrachten; STRAUß (1986a) versteht unter dem Begriff der kommunikativen Verfahren

Aktivitäten/Handlungsfolgen, die - von den Beteiligten - unter einer gemeinsamen Zielsetzung begriffen werden, als Antworten auf spezifische Problemsituationen fungieren und der Handlungskoordination zum Zweck der Problemlösung dienen. ${ }^{107}$

Dabei ist zwischen (i) den "Makroverfahren" als generelle sprachliche Handlungsformen auf makrostruktureller Ebene und (ii) deren speziellen unterschiedlichen Ausprägungen auf mikrostruktureller Ebene, den „Mikroverfahren “108, zu unterscheiden. ${ }^{109}$

Die auf den Machtanspruch konzentrierte Basisfunktion der politischen Sprachverwendung kann sehr verschiedene Ausprägungen haben. Grundsätzlich muss dabei zwischen den Polen argumentativ-rationales ÜBERZEUGEN und eher gefühlsmäßig-rhetorisches ÜBERREDEN differenziert werden. Da auch dem ÜBERREDEN/ÜBERZEUGEN unterschiedliche Zielsetzungen zugrunde liegen können, differenziert GRÜNERT (1983) wiederum zwischen vier Diskurstypen. Bevor die Analyse der Parteiprogramme im Zentrum der Betrachtungen steht, sollen in den nachfolgenden Kapiteln diese vier Sprachspiele mit den einhergehenden konstitutiven Sprachfunktionen jeweils dargestellt werden.

106 Herrgen, Joachim: Die Sprache der Mainzer Republik (1792/93). Historischsemantische Untersuchungen zur politischen Kommunikation. Tübingen 2000, S. 42

107 Strauß 1986a, S. 8

108 Strauß 1986a, S. 10

109 In Anlehnung an die sprachwissenschaftliche Forschungsliteratur und an eine in sprechhandlungstheoretischen Untersuchungen übliche Konvention sollen auch in dieser Untersuchung Majuskeln für die Beschreibung der Makro- und Mikroverfahren verwendet werden. 


\subsection{Das regulative Sprachspiel/ Die regulative Sprachfunktion}

In der regulativen oder „legislativ-regulativen “110 Sprachfunktion werden die Beziehungen zwischen Regierenden und Regierten von oben nach unten akzentuiert; daher bildet diese Funktion die sich sprachlich manifestierende und jeweils gültige Herrschaftsstruktur ab. Der Verfassungstext verkörpert den "regulierenden Text par excellence ${ }^{\star 111}$, denn insbesondere in diesem Texttyp ist "das Mikroverfahren ,NORMENSETZEN' in reiner Form verwirklicht. “112

Auf den Terminus des kommunikativen Verfahrens wird an späterer Stelle ausführlich eingegangen; weiterhin wird die hier vorgestellte Sprachfunktion erneut aufgegriffen, wenn das Godesberger Programm mit dem Grundgesetz als regulierendem Text verglichen wird. Als typische Textformen neben dem Grundgesetz als Verfassungstext, in denen diese Sprachfunktion erscheint, nennt HERRGEN (2000) „Gesetze, Verordnungen, Dekrete, Erlasse, oder Befehle. ${ }^{\prime 113}$ Der Liste können weitere normative Texte wie Verfügungen, Edikte, Bullen, Gebote, Verbote, Mahnungen und Anweisungen hinzugefügt werden. Von der Öffentlichkeit werden regulierende Verfahren häufig negativ eingeschätzt, was zu protestierenden Verfahren der Öffentlichkeit führen kann, die ihrerseits von den Behörden oder Institutionen negativ eingeschätzt werden.

\subsection{Das instrumentale/begehrende Sprachspiel/ Die poskative Sprachfunktion}

Die „poskative Sprachfunktion“114 akzentuiert die Beziehungen zwischen Regierenden und Regierten in umgekehrter Richtung zur regulativen Sprachfunktion. Sie repräsentiert Wünsche oder Forderungen von verschiedenen nicht unmittelbar an der Herrschaft beteiligten Gruppierungen, Klassen oder Gesellschaftsschichten gegenüber den Machthabern. Aufrufe, Flugblätter oder Manifeste sind die Texttypen, in denen dieses „instrumentale/begehrende Sprachspiel“115 zum Ausdruck kommt, da durch sie aufgezeigt wird, an welchen Stellen ein regulatives Sprachspiel (z.B. Gesetzentwurf) der Korrektur bedarf. Das kommunika-

110 Klein, Josef: „Politische Textsorten.“ In: Germanistische Linguistik (106-107). Hg. v. Klaus Brinker. Hildesheim/Zürich/New York 1991a, S. 247

111 Strauß 1986a, S. 40

112 Strauß 1986a, S. 40

113 Herrgen 2000, S. 44

114 Herrgen 2000, S. 44

115 Grünert 1983, S. 51 
tive Verfahren PROTESTIEREN wird durch folgende Sprachhandlungen realisiert:

Hier werden gegenüber der institutionellen Herrschaft Wünsche geäußert, Bitten vorgetragen, Petitionen eingereicht, Forderungen gestellt, Ansprüche erhoben, Gegenentwürfe formuliert, und gegenüber unrechtmäßiger oder unrechtmäßig empfundener Macht wird Widerstand geleistet. ${ }^{116}$

Durch diese Sprachfunktion versuchen daher Einzelne oder Gruppen, Anteil an Herrschaft zu gewinnen oder Widerstand $\mathrm{zu}$ leisten. Die poskative Sprachfunktion bestärkt weiterhin erneut die bereits diskutierte These, dass sprachliches Handeln in Taten, Aktivitäten und Handlungen eingebettet ist; so verbindet sich diese Sprachfunktion mit "Revolutionen und Aufständen, mit Protestationen, Demonstrationen, Bürgerinitiativen." 117

\subsection{Das integrative Sprachspiel/ Die integrative Sprachfunktion}

Die integrative Sprachfunktion wird in der Regel „in Texten wie Parteiprogrammen, politischen Liedern, Parteitagsreden usw. ${ }^{\prime 118}$ sowie in panegyrischen Gedichten verwirklicht, die eine identifikatorische Funktion für die Gruppenmitglieder haben. Es geht in diesem Sprachspiel daher um einen "gruppeneinigenden Proze ${ }^{\prime \prime 119}$ und um (Gruppen-)Solidarität. Mit solchen Texten definiert sich die Gruppe, stabilisiert sich nach innen und grenzt sich nach außen ab; die Abgrenzung, beispielsweise gegenüber dem politischen Gegner, wird bei KAACK (1971) mit dem Terminus Profilfunktion bezeichnet (vgl. 2.1.2.2).

Dem Wortschatz kommt hier eine besondere signalisierende Wirkung zu. Symptomatisch für integrative Sprachspiele ist "der Gebrauch von Schlagwörtern, Fahnen- und Stigmawörtern [...], die für die Mitglieder von Gruppen und Parteien die Funktion von ideologieidentifizierenden Symbolen übernehmen. ${ }^{\prime 120}$

\footnotetext{
116 Grünert 1983, S. 51

117 Grünert 1983, S. 51

118 Herrgen 2000, S. 45

119 Grünert 1983, S. 53

120 Strauß 1986a, S. 7
} 


\subsection{Das informativ-persuasive Sprachspiel/ Die informativ-persuasive Sprachfunktion}

Durch das Abzielen auf Bewusstseinsbildung, Motivation und Begründung kann man die informativ-persuasive Sprachfunktion primär in der kommerziellen Werbung (im Zusammenhang dieser Untersuchung in politischen Werbetexten) realisiert sehen. Das informativ-persuasive Sprachspiel zeichnet sich durch sein breites Textspektrum von politischen Gedichten über Traktate, Pamphlete oder Flugblätter bis zu Bundestagsreden und Wahlprogrammen aus und bildet "mit seinen Entscheidungshilfen gebenden, meinungsbildenden Texten nahezu vollständig das öffentliche Bild der gegenwärtigen politischen Kommunikation “121 ab und ist daher "nicht, wie das regulative und das instrumentale Sprachspiel, unmittelbar in politisches Handeln verflochten, sondern es will bei seinen Adressaten ein bestimmtes Bewußtsein erzeugen, es will Meinungen steuern, es will werben. “122 Die persuasiven Effekte sind dabei die Meinungsbildung sowie deren Stabilisierung und Beeinflussung, d.h. konkret die
Zustimmung für politische Ziele, Programme, Maßnahmen zu erlan- gen - für eine zukünftige Politik, die planend vorgeschlagen oder ge- fordert wird, oder für eine vollzogene Politik, die nachträglich erklärt, begründet, gerechtfertigt, verteidigt wird. ${ }^{123}$

Zwar ist eine reine Informationskundgabe ohne persuasive Zielsetzung denkbar; für den Kommunikationsbereich der Politik ist jedoch keine Persuasion denkbar, die ohne informative Komponenten auskommt. Daher ist die persuasive Sprachverwendung „nicht im Gegensatz zu ,Information', zu ,informativem Sprechen' zu verstehen [...], sondern im Zusammenhang von Legitimation und LEGITIMIEREN als ,Zustimmung erlangen für eine zukünftige oder vollzogene Politik'." ${ }^{124}$ Persuasives Sprechen ist jedoch nicht auf die politische Sprachverwendung beschränkt. Die Persuasion stellt vielmehr eine allgemeine Funktion in der Sprachverwendung dar.

Nach GRÜNERT (1983) erscheint das informativ-persuasive Sprachspiel in den drei Varianten (i) politische Theorie („,die theoretisch-reflektierenden und prospektiven Modelle und Entwürfe gesellschaftlicher Organisation bis hin zur Utopie ebenso wie die empirischen Analysen politischer Ord-

\footnotetext{
$121 \quad$ Strauß 1986a, S. 7 
nungen und Prozesse“125), (ii) praktische Information (,,alle jene Informationsprozesse [...], die der unmittelbaren Vorbereitung des regulativen Sprachspiels dienen“ und "nötig sind, um politische Entscheidungen zu ermöglichen "126) und (iii) Propaganda („,der eigentlich werbende, propagandistische Strang" ${ }^{\prime 127}$ ).

Die persuasive Funktion ist als Rahmenbestimmung zu betrachten, da ihr verschiedene sprachliche Handlungsformen untergeordnet werden können.

Da die unterschiedlichen Handlungsformen sowohl mit negativen Ausdrücken (LÜGEN, ÜBERREDEN, TÄUSCHEN, MANIPULIEREN) als auch mit positiven Ausdrücken (INFORMIEREN, ÜBERZEUGEN, ERKLÄREN, BEGRÜNDEN, ARGUMENTIEREN) belegt sein können, ist der Ausdruck persuasiv aufgrund der lateinischen Bedeutung von persuadere zwar vorgezeichnet (überreden, überzeugen), in diesem $\mathrm{Zu}$ sammenhang ist der Ausdruck allerdings als wertneutrale Bezeichnung zu betrachten:

Als persuasives Sprechen wird nur ausgesagt, daß es die Funktion hat, Zustimmung beim Bürger zu erlangen, nichts darüber, auf welche Weise, mit welchen positiv oder negativ bewerteten Mitteln der politisch Handelnde die Zustimmung zu erlangen sucht. ${ }^{128}$

Aufgrund der vorangegangenen Betrachtung der unterschiedlichen Sprachfunktionen und die Diskussion um die Fachsprachlichkeit soll dieses Kapitel mit einer abschließenden Definition des Ausdrucks Politische Sprache beendet werden. STRAUß (1986c) versteht darunter die "Gesamtheit der Eigenschaften des Sprachgebrauchs im Bereich von Staat, Gesellschaft und Öffentlichkeit, also die Gesamtheit der Regeln und Mittel für die in diesem Bereich produzierten Sprachspiele, kommunikativen Verfahren, Textsorten und für den Gebrauch spezieller syntaktischer Verfahren und Wortschatzklassen “129 und liefert folgende Definition:

Politische Sprache ist ein spezifischer Verbund aus Fachsprachenpluralität und öffentlicher Sprache, eine Sprache, deren soziale Geltung nicht auf bestimmte Expertengruppen eingeschränkt ist und die nicht

\footnotetext{
125 Grünert 1983, S. 55

126 Grünert 1983, S. 56

127 Grünert 1983, S. 56

128 Dieckmann 1981, S. 139

129 Strauß, Gerhard: „Schwere Wörter in der Politik.“ In: Der politische Wortschatz. Zur Kommunikations- und Textsortenspezifik. Hg. v. Gerhard Strauß. Tübingen 1986c, S. 194
} 
nur über ein kognitiv-denotatives, sondern auch ein evaluativpersuasives Potential verfügt. ${ }^{130}$

\subsubsection{Funktionen von Parteiprogrammen}

Nachfolgend sollen die Funktionen der politischen Sprachverwendung auf die Parteiprogramme als spezifische Ausprägung der politischen Sprache übertragen werden, so lassen sich dem Parteiprogramm bestimmte Außenwirkungen formulieren und Funktionen zuschreiben. ${ }^{131}$

Durch das Parteiprogramm werden die politische Orientierung und die Marschrichtung der Partei verdeutlicht. Weiterhin spiegelt es die strategischen und taktischen Überlegungen der jeweiligen Partei wider. Die Abgrenzung von den konkurrierenden Parteien ist eine weitere wichtige Funktion (integrative Funktion oder Profilfunktion); aus dieser resultiert die Funktion der direkten gegnerischen Bezugnahme (Agitationsfunktion):

Aus der Abgrenzung gegenüber den anderen Parteien und aus dem grundsätzlichen Konkurrenzverhältnis ergibt sich, daß die Parteiprogramme auch die Funktion der Vorbereitung der Auseinandersetzung mit den anderen Parteien haben $[\ldots] .{ }^{132}$

Das Parteiprogramm soll neben der Bereitstellung von Informationen in der Lage sein, Wähler von der eigenen Weltanschauung, d.h. von den parteispezifischen Ansichten, zu überzeugen und zu gewinnen (informativ-persuasive Sprachfunktion). Aus der unabdingbaren Werbefunktion resultiert die Meinung, "daß Parteiprogramme hauptsächlich dazu dienen, Wähler anzulocken, aber nicht dazu, die künftige Politik zu skizzieren $[\ldots]^{\prime \prime} 133$; dieser Funktion kommt insbesondere in der heutigen Medienlandschaft eine hohe Bedeutung zu. Der Einfluss der Massenmedien ist in diesem Zusammenhang signifikant:

Schlagwörter wie ,Mediengesellschaft', ,Zuschauerdemokratie' oder ,Telekratie' zeugen von der verbreiteten Auffassung, daß Medien in der Politik eine bedeutende Rolle spielen bzw. von den politischen

130 Strauß 1986c, S. 194

131 Einige der in diesem Kapitel dargestellten Funktionen wurden bereits im Zuge der Differenzierung nach den Parteiprogrammtypen erwähnt oder gestreift. Es soll hier trotzdem ausführlich auf die unterschiedlichen Funktionen eingegangen werden, da in den nachfolgenden Kapiteln untersucht werden soll, ob und inwiefern sich einige dieser Grundannahmen in der Sprachhandlungsanalyse bestätigt finden.

132 Kaack 1971, S. 402

133 Edelman, Murray: Politik als Ritual. Die symbolische Funktion staatlicher Institutionen und politischen Handelns. Frankfurt/Main 1976, S. 6 
Akteuren immer stärker als Kampfmittel im Prozeß ,symbolischer Politik' instrumentalisiert werden. ${ }^{134}$

Das Parteiprogramm muss daher in der Lage sein, die eigene Partei positiv darzustellen, wenn auch nur für einen begrenzten Zeitraum. Insbesondere der Wechselwähler muss durch das Parteiprogramm angelockt werden, denn

die Mehrheit der Wahlberechtigten besteht mittlerweile nicht mehr aus Stammwählern und erst recht nicht aus treuen Parteianhängern. Im Zuge ihrer Entwicklung, die Soziologen und Politologen mit Begriffen wie ,Individualisierung', ,Wertewandel', ,Entideologisierung' und ,Stimmungsdemokratie' gekennzeichnet haben, ist die Rolle der Partei als politische Heimat der Rolle als politisches Dienstleistungsunternehmen gewichen. ${ }^{135}$

Das Anhängerschaftsmodell wird somit durch „die Dominanz des Marktmodells der politischen Kommunikation"136 abgelöst; demnach kann die Politik wie ein Warenmarkt verstanden werden, bei dem die Wähler die Rolle des Verbrauchers und die politischen Parteien die Rolle der Anbieter spielen. Nach diesem Modell müssen die Parteien ein politisches Angebot in ihren Programmen niederlegen; dementsprechend kann diese Funktion mit dem Terminus der "politischen Offerte ${ }^{\prime 137}$ beschreiben werden:

Wir sehen Parteiprogramme als die zweckmäßige Form an, in der die Partei ihren Beitrag leistet zur Schaffung der objektiven Transparenz des politischen Angebots in der Demokratie. ${ }^{138}$

Dabei bilden die Programme „die Operationsbasis für die Sachforderungen der Parteien, die Basis, von der aus die Parteien in die Auseinandersetzungen um die Konkretisierung der politischen Forderungen gehen. ${ }^{139}$ Das Parteiprogramm hat aber nicht nur die Aufgabe, nach außen zu wirken. Als spezifische Innenwirkung hat es die Aufgabe, das innere

134 Holly, Werner: „Hier spricht der Zuschauer. Ein neuer methodischer Ansatz in der sprachwissenschaftlichen Erforschung politischer Fernsehkommunikation." In: Sprachstrategien und Dialogblockaden. Linguistische und politikwissenschaftliche Studien zur politischen Kommunikation. Hg. v. Josef Klein/Hajo Dieckmannshenke. Berlin/New York 1996, S. 103

135 Klein, Josef: „Dialogblockaden. Dysfunktionale Wirkungen von Sprachstrategien auf dem Markt der politischen Kommunikation." In: Sprachstrategien und Dialogblockaden. Linguistische und politikwissenschaftliche Studien zur politischen Kommunikation. Hg. v. Josef Klein/Hajo Dieckmannshenke. Berlin/New York 1996b, S. 5

136 Klein 1996b, S. 5

137 Flohr 1968, S. 41

138 Flohr 1968, S. 45

139 Kaack 1971, S. 402 
Gefüge der Parteien zu gestalten; es ist daher als gemeinsame verbindliche Richtschnur, als Anleitung für die in Regierungspositionen stehenden Parteimitglieder hinsichtlich deren politischem Handeln und Denken $\mathrm{zu}$ verstehen (Integrationsfunktion). Darüber hinaus soll es bewirken, dass die Mitglieder sich mit ihrer Partei und deren Zielen identifizieren können (Identifikationsfunktion). Vor allem aber muss das Parteiprogramm die Ziele seiner Partei verbindlich nennen und die zukünftige Politik transparent machen:

Es sollte Aufgabe der Parteien sein, deutlich zu machen, welche Politik von ihnen im Falle des Wahlsiegs betrieben wird. Sie haben dem Bürger die von ihnen verfolgten Ziele kundzutun und ihm auf diese Weise die Informationen anzubieten, die er braucht, um sich rational für eine der angebotenen Richtungen entscheiden zu können. Dabei kommt es entscheidend darauf an, daß die künftige Regierungspolitik klargemacht wird. ${ }^{140}$

Letztlich soll nicht unerwähnt bleiben, dass ein Parteiprogramm auch eine Legitimitätsfunktion erfüllt, denn „wer sich darauf berufen kann, im Sinne des Parteiprogramms gehandelt zu haben, gilt als legitimiert. " ${ }^{141}$

Die unterschiedlichen Funktionen lassen sich nach folgendem Schema unterteilen, das in seiner Darstellung die verschiedenen Typen von Parteiprogrammen ebenso berücksichtigt. Dabei wird die Intensität angegeben, mit der die einzelnen Funktionen von den jeweiligen Programmtypen erfüllt werden. Die Skala reicht von $X$ als geringstem Intensitätsgrad bis XXX als maximalem Intensitätsgrad.

\begin{tabular}{|l|l|l|l|l|}
\hline Funktionen & $\begin{array}{c}\text { Grundsatz- } \\
\text { programm }\end{array}$ & $\begin{array}{c}\text { Aktions- } \\
\text { programm }\end{array}$ & $\begin{array}{c}\text { Wahlpro- } \\
\text { gramm }\end{array}$ & $\begin{array}{c}\text { Regierungs- } \\
\text { programm }\end{array}$ \\
\hline a) nach außen & & & & \\
\hline 1. Werbungsfunktion & $\mathrm{X}$ & $\mathrm{XX}$ & $\mathrm{XXX}$ & $\mathrm{XX}$ \\
\hline 2. Profilfunktion & $\mathrm{XX}$ & $\mathrm{XXX}$ & $\mathrm{XXX}$ & $\mathrm{XXX}$ \\
\hline 3. Agitationsfunktion & $\mathrm{X}$ & $\mathrm{XX}$ & $\mathrm{XXX}$ & $\mathrm{XX}$ \\
\hline 4. Operationsbasis & $\mathrm{X}$ & $\mathrm{XX}$ & $\mathrm{XX}$ & $\mathrm{XXX}$ \\
\hline b) nach innen & & & & \\
\hline 1. Integrationsfunktion & $\mathrm{XXX}$ & $\mathrm{XX}$ & $\mathrm{XX}$ & $\mathrm{X}$ \\
\hline 2. Identifikationsfunktion & $\mathrm{XXX}$ & $\mathrm{X}$ & $\mathrm{X}$ & $\mathrm{X}$ \\
\hline 3. Stimulationsfunktion & $\mathrm{X}$ & $\mathrm{XXX}$ & $\mathrm{XXX}$ & $\mathrm{X}$ \\
\hline 4. Herrschaftsfunktion & $\mathrm{XX}$ & $\mathrm{XX}$ & $\mathrm{XXX}$ & $\mathrm{XX}$ \\
\hline 5. Legitimationsfunktion & $\mathrm{XXX}$ & $\mathrm{XX}$ & $\mathrm{X}$ & $\mathrm{X}$ \\
\hline
\end{tabular}

Abbildung 3: (vgl. KAACK 1971, Tabelle 117, S. 403)

$140 \quad$ Flohr 1968, S. 41

141 Kaack 1971, S. 403 
Wenn die drei bereits dargestellten Typen von Parteiprogrammen auch unterschiedlich sein mögen und verschiedene Funktionen zu erfüllen haben, so verbindet sie als Anleitungstexte dennoch ein gemeinsamer Zweck: Die gemeinsame Funktion aller drei Programmtypen ist die Strukturierung der politischen Willensbildung innerhalb einer Partei nach bestimmten Grundsätzen. Den Willensbildungs- und Entscheidungsprozessen sind Meinungsbildungsprozesse als kommunikative Prozesse vorgelagert; dieser letztgenannte Prozess gehört „,zum unstrukturierten Bereich der politischen Kommunikation, während Formen der Willensbildung an Organisationen gekoppelt und zumeist formalisiert sind (so die innerparteiliche Willensbildung). ${ }^{.142}$ Im Zuge eines Meinungsbildungsprozesses werden politische Bedürfnisse artikuliert und öffentlich diskutiert; solche Prozesse ermöglichen auf diese Weise die Umsetzung der bereits erwähnten poskativen Sprachfunktion und sind daher "für die Herausbildung von kollektiven Interessen wie für die Interessenanmeldung und -durchsetzung gegenüber politischen Akteuren von Bedeutung. ${ }^{\prime 143}$

Das Parteiprogramm, insbesondere das Wahlprogramm, stellt eine auf die Schriftsprache fixierte, medienvermittelte Interaktion zwischen der Partei als Senderkollektiv sowie den Bürgern und Parteimitgliedern als Empfängerkollektive dar. Die pragmatische Analyse des Godesberger Programms und der anderen Programme beschäftigt sich daher intensiv mit den Absichten des Senders.

Neben den oben dargestellten strategischen und politischen Funktionen, die das Parteiprogramm als solches haben kann oder muss gibt es jedoch unter dem sprachhandlungstheoretischen Aspekt auch eine kommunikative Funktion. Die kommunikative Funktion bezieht sich auf das Mitgeteilte und das Gemeinte unter der Fragestellung, wie sich das, was in der jeweiligen Situation über bestimmte Dinge ausgesagt wird, zu dem verhält, was der Produzent der sprachlichen Handlung damit dem Empfänger gegenüber beabsichtigt; die Analyse des absichtsvollen Einwirkens auf den Rezipienten kann als Kernstück der linguistischen Pragmatik verstanden werden. Die Verwendung der Sprache in der Politik ist als Handeln zu betrachten, das Folgen auf das Verhältnis zwischen den jeweiligen Kommunikationspartnern hat; "Äußerungen sind absichtsvolle Sprachverwendungen in konkreten gesellschaftlichen Situationen. “144

142 Jarren, Otfried/Sarcinelli, Ulrich/Saxer, Ulrich (Hrsg.): Politische Kommunikation in der demokratischen Gesellschaft. Ein Handbuch mit Lexikonteil. Wiesbaden 1998, S. 687/688

143 Jarren/Sarcinelli/Ulrich 1998, S. 688

144 Bachem 1979, S. 18 
Das folgende Kapitel unternimmt nun den Versuch, das Godesberger Programm und die anderen Parteiprogramme hinsichtlich der politischen Sprachverwendung zu charakterisieren, funktional zu klassifizieren und auf den Aspekt der Fachsprachlichkeit hin zu untersuchen; „ein Parteiprogramm hat primär andere Aufgaben als die Befriedigung ästhetischer Bedürfnisse: es hat bestimmte politische Funktionen zu erfüllen. “145 Mit diesen Funktionen soll nun die Untersuchung der Sprachverwendung beginnen.

\subsection{Wahl-/Aktionsprogramme}

In Bezug auf das Wahlprogramm kann das komplexe Handlungsmuster AKTIVIEREN als dominierendes Makroverfahren betrachtet werden: „Bei seiner Realisierung hat einer der Akteure vorkommunikativ schon einen Handlungsplan und es geht darum, den anderen zu bewegen, sich entsprechend diesem Handlungsplan zu verhalten. ${ }^{\text {} 146}$

Das Makroverfahren AKTIVIEREN kann nach den positiven Ausprägungen ARGUMENTIEREN und ÜBERZEUGEN als Mikroverfahren differenziert werden; letztere sind der Text- und Sprechaktebene bedeutend näher und können daher als konkrete Realisierungen des oben erwähnten Makroverfahrens betrachtet werden. Die negativen Ausprägungen als Persuasion stellen die Mikroverfahren WERBEN und ÜBERREDEN dar.

Im Hinblick auf das Makroverfahren AKTIVIEREN ist es wenig wahrscheinlich, dass in einem Wahlprogramm die Fernziele der Partei angegeben werden; da es für den Wähler um das beabsichtigte und unmittelbar bevorstehende Handeln der Partei und weniger um die Sichtweisen geht, wird der Schwerpunkt auch nicht auf der gesellschaftspolitischen Analyse liegen. Da sich bestimmte Forderungen, besonders solche, die mit Einschränkungen des Wählers einhergehen, nur schwer mit der Werbefunktion vereinbaren lassen, gehören Forderungen eher in den Bereich der Grundsatzprogramme.

Die Ziele der Partei müssen klar und deutlich formuliert sein, da die Auseinandersetzung mit den unterschiedlichen Zielvorstellungen - insbesondere in der Gegenüberstellung mit denen der konkurrierenden Parteien - nur dann gelingen kann, wenn sie als solche im Programm erkennbar sind; „vage Floskeln lassen das Gewollte im dunkeln und geben dem Wähler kein deutliches Bild“147, und vor allem „sollte man sie nicht unauffällig in die bloßen Beschreibungen und Erklärungen

\footnotetext{
145 Schönbohm 1974, S. 17

146 Strauß 1986a, S. 10

147 Flohr 1968, S. 46
} 
schmuggeln, gar getarnt durch eine sprachliche Formulierung, die verbirgt, daß es sich in Wahrheit um eine Stellungnahme handelt. " 148

Diese Vorgabe wirkt sich auf die Wahl der sprachlichen Handlungen und die kommunikativen Verfahren eines Parteiprogramms aus; dabei sollte es

unmißverständlich sein, welche Aufgabe die Aussage hat, welchen Anspruch man mit ihr verbindet, ob man etwa beschreiben, erklären, prognostizieren, fordern oder versprechen will. ${ }^{149}$

Die kommunikativen Verfahren eines Wahlprogramms können einer Hierarchie folgend untergliedert werden; sind derart gewählt und angeordnet, dass sie als ein aufeinander einwirkendes dynamisches System bestimmte Ziele erreichen (Makro- und Mikroverfahren):

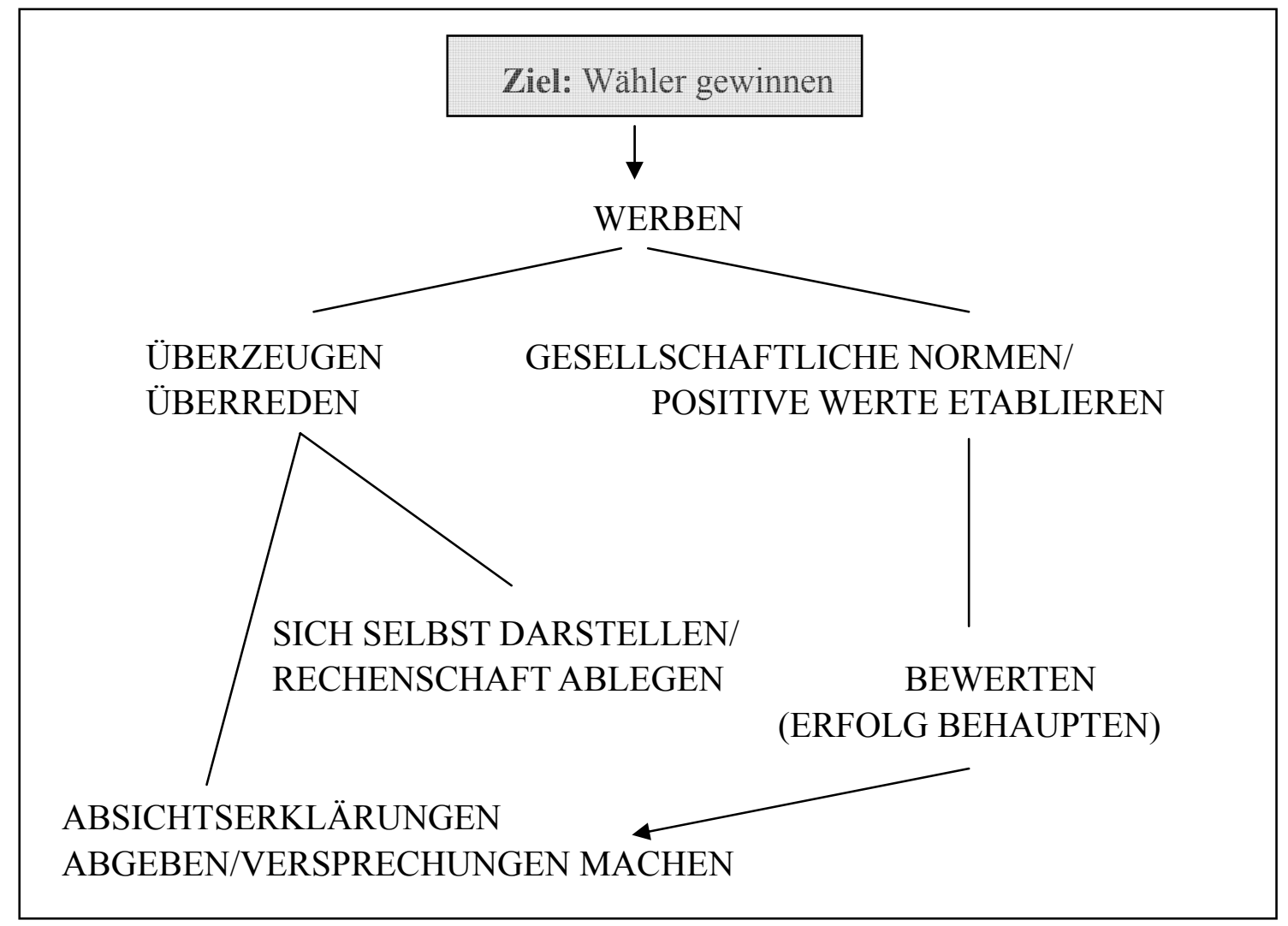

Abbildung 4: (vgl. STRAUB 1986a, S. 45)

148 Flohr 1968, S. 70

149 Flohr 1968, S. 70 


\subsection{Grundsatzprogramme}

Die Handlungsziele des Grundsatzprogramms weichen erwartungsgemäß von der oben dargestellten Abbildung ab. Charakteristische Handlungsziele sind folglich die Partei zu stabilisieren, zu integrieren und Konsens herzustellen. Hinsichtlich des Grundsatzprogramms kann das komplexe Handlungsmuster SOLIDARISIEREN und INTEGRIEREN als Makroverfahren nach den Ausprägungen NORMEN UND WERTE ETABLIEREN und HANDLUNGSPLÄNE ENTWICKELN als Mikroverfahren differenziert werden.

Die oben dargestellten Makroverfahren werden bei der Betrachtung der innerpolitischen Rahmenbedingungen der SPD deutlich, als diese im Jahre 1987 einen neuen Grundsatzprogramm-Entwurf veröffentlicht. Die SPD stand dabei vor großen Problemen: Bruch der rot-grünen Koalition in Hessen 1987 und Niederlage bei den vorgezogenen Landtagswahlen; komplizierte Ausgangsbedingungen für die Wahlen in Hamburg, Rheinland-Pfalz, Schleswig-Holstein und Bremen; Führungskrise; überraschender Rücktritt Willy Brandts vom Parteivorsitz; Ankündigung eines Sonderparteitags mit der Wahl Hans-Jochen Vogels zum neuen Vorsitzenden und Oskar Lafontaines neben Johannes Rau zu einem seiner Stellvertreter. Daher musste sich die SPD

offenkundig auf eine längere und schmerzhafte Phase komplizierter politischer Neuorientierung einstellen. [...] Dies wird die innerparteiliche Diskussion und die ohnehin sehr harten internen Konflikte intensivieren und verschärfen. In einer solchen Situation erhalten auch programmatische Grundsatzdokumente eine besondere Bedeutung. ${ }^{150}$

Abschließend kann domit festgehalten werden: Ein bestimmtes Kapitel des Godesberger Programms, das ein spezielles Thema aufgreift, ist ein Produkt innerhalb der Sprachspiele Politische Werbung oder Parteiinterne politische Meinungsbildung in Realisierung bestimmter kommunikativer Verfahren, beispielsweise AKTIVIEREN, INTEGRIEREN oder SOLIDARISIEREN, mit der Möglichkeit der Doppelung durch Realisierung komplementärer Verfahren, wie beispielsweise WERBEN, ERKLÄRUNGEN ABGEBEN oder NORMEN UND WERTE ETABLIEREN.

150 Institut für Marxistische Studien und Forschungen e.V. (IMSF) (Hrsg.): SPD wohin? Zur Analyse und Kritik des SPD-Grundsatzprogrammentwurfs. Informationsbericht Nr. 44. Frankfurt/Main 1987, S. 5 


\subsection{Wahlprogramm vs. Grundsatzprogramm}

Wie bereits erwähnt werden die langfristigen Ziele eines Grundsatzprogramms durch das Wahlprogramm präzisiert und auf die Realisierungsmöglichkeiten innerhalb der nächsten Legislaturperiode zugeschnitten. Wenn die SPD in ihrem Godesberger Programm fordert, dass die Versorgung der Bevölkerung mit Wohnraum gewährleistet werden muss, begründet sie diese Forderung mit dem Recht des Menschen auf Wohnraum und mit der sozialen Verpflichtung der Partei gegenüber den Bürgern/Wählern. Das Wahlprogramm dagegen ist derart konzipiert, dass es eine solche Äußerung mit statistischen Zahlen und solchen Fakten unterlegt, die Aussagen darüber zulassen, was bereits durch die Arbeit der Partei erreicht wurde; durch die Vermittlung von Fakten und Daten wird das Makroverfahren INFORMIEREN realisiert.

Weiterhin werden dadurch die Voraussetzungen für die Meinungs- und Bewusstseinsbildung geschaffen und die Überzeugungs- und Werbefunktion erfüllt. Diese Werbefunktion stimmt jedoch nicht mit den Schwerpunkten des Grundsatzprogramms überein. Allerdings: Auch der konkrete Programmtyp des Wahlprogramms kommt nicht umhin, die abstrakten Forderungen eines Grundsatzprogramms - wenn auch inhaltlich kürzer - aufzugreifen, gesellschaftspolitisch oder sozialphilosophisch einzuordnen sowie in den Zusammenhang der Gesamtprogrammatik einzuordnen:

Je mehr auch ein Aktionsprogramm den Zusammenhang der einzelnen Ziele, ihren Stellenwert in der verfolgten Konzeption und letztlich in der Gesamtauffassung des politischen Lebens erkennen läßt, desto geringer ist die Gefahr, daß sich der Wähler von einem einzelnen Ziel, das energisch propagiert wird, zu einer vorschnellen Wahlentscheidung hinreißen läßt. ${ }^{151}$

Die nachfolgende Tabelle veranschaulicht exemplarisch, wie sich die Konzeptionen von Wahl- und Grundsatzprogramm unterscheiden. Dabei soll das Beispiel der Wohnraumversorgung sowie der medizinischen Versorgung aufgegriffen werden. Aus Platzgründen wurde der Text des Wahlprogramms verkürzt wiedergegeben, die Ausführungen sind umfangreicher. Deutlich wird dennoch, dass das Wahlprogramm zur Konkretisierung statistische Fakten anbringt und konkrete Maßnahmen erwähnt:

151 Flohr 1968, S. 96 


\begin{tabular}{|c|c|}
\hline $\begin{array}{c}\text { Godesberger } \\
\text { Programm (1959) }\end{array}$ & $\begin{array}{c}\text { Wahlprogramm der SPD } \\
\text { (13. Oktober 1972) }\end{array}$ \\
\hline $\begin{array}{l}\text { Jeder hat ein Recht auf } \\
\text { eine menschenwürdige } \\
\text { Wohnung. Sie ist die } \\
\text { Heimstätte der Familie. } \\
\text { Sie muß deshalb auch } \\
\text { weiterhin sozialen } \\
\text { Schutz genießen und } \\
\text { darf nicht nur privatem } \\
\text { Gewinnstreben über- } \\
\text { lassen werden. } \\
\text { Die Wohnungs-, Bau- } \\
\text { und Bodenpolitik muß } \\
\text { den Mangel an Wohn- } \\
\text { raum beschleunigt } \\
\text { beheben. Der soziale } \\
\text { Wohnungsbau ist zu } \\
\text { fördern. Der Mietzins } \\
\text { ist nach sozialen Ge- } \\
\text { sichtspunkten zu beein- } \\
\text { flussen. Die Bodenspe- } \\
\text { kulation ist zu } \\
\text { unterbinden, unge- } \\
\text { rechtfertigte Gewinne } \\
\text { aus Bodenverkäufen } \\
\text { sind abzuschöpfen. } \\
\text { (S. 80) }\end{array}$ & $\begin{array}{l}\text { [...] Ein preisbegrenzendes Vorkaufsrecht, mit } \\
\text { dem die Gemeinden Spekulationskäufe unter- } \\
\text { binden und zum Verkauf anstehende } \\
\text { Grundstücke schneller erwerben können. [...] } \\
\text { Die Bodenwertzuwachssteuer soll die Spekula- } \\
\text { tion und damit ein übermäßiges Ansteigen der } \\
\text { Bodenpreise verhindern. (S. 104) } \\
\text { Vor allem in Verdichtungsregionen wurden die } \\
\text { kinderreichen Familien und auch junge Ehe- } \\
\text { paare unerträglich benachteiligt. } \\
\text { Wir haben daher eine Wende der Wohnungspo- } \\
\text { litik herbeigeführt: Der Wohnungsbau steigt. } \\
1970 \text { wurden rund } 500 \text { 000 Wohnungen fertig- } \\
\text { gestellt, } 1971 \text { rund } 550 \text { 000 (darunter 180 000 } \\
\text { Ein- und Zweifamilienhäuser und } 70 \text { 000 Eigen- } \\
\text { tumswohnungen). } 1972 \text { werden es mehr als } 600 \\
\text { 000 sein. } \\
\text { Wir haben durch das langfristige Wohnungs- } \\
\text { bauprogramm die Zahl der geförderten Sozial- } \\
\text { wohnungen und Heimplätze bis } 1972 \text { um } 20 \% \\
\text { auf über 200 000 pro Jahr gesteigert. } \\
\text { Wir wollen auch in den kommenden Jahren } \\
\text { mindestens } 200 \text { 000 bis } 250 \text { 000 Sozialwohnun- } \\
\text { gen fördern. } \\
{[\ldots . .] \text { Die neuen mietrechtlichen Bestimmungen }} \\
\text { haben sich bewährt, sie schützen den ver- } \\
\text { tragstreuen Mieter vor willkürlichen Kündi- } \\
\text { gungen und ungerechtfertigten Mieterhöhun- } \\
\text { gen. (S. 105) }\end{array}$ \\
\hline $\begin{array}{l}\text { Es ist eine öffentliche } \\
\text { Aufgabe, die Kranken- } \\
\text { hausversorgung sicher- } \\
\text { zustellen. (S. 79) }\end{array}$ & $\begin{array}{l}\text { Die Versorgung der Bevölkerung mit leistungs- } \\
\text { fähigen Krankenhäusern wird durch das Kran- } \\
\text { kenhausfinanzierungsgesetz verbessert. Bund, } \\
\text { Länder und Gemeinden tragen gemeinsam die } \\
\text { Investitionskosten. } 1972 \text { hat der Bund dafür } 720 \\
\text { Mill. DM aufgebracht, } 1973 \text { werden es } 890 \text { Mill. } \\
\text { DM sein. Die Beiträge werden weiter steigen. } \\
\text { (S. 101) }\end{array}$ \\
\hline
\end{tabular}




\subsubsection{Das Spektrum der sprachlichen Handlungsformen}

Die pragmatische Analyse versucht nun, die Äußerungen des Godesberger Programms als Grundsatzprogramm auf deren absichtsvolle Aussagen, wie Behauptungen, Versprechen, Aufforderungen oder Appelle, Anklagen oder Vorwürfe sowie Forderungen zu untersuchen und darzustellen. ${ }^{152}$ Die jeweiligen Untersuchungsschwerpunkte der sprachhandlungstheoretischen Analyse ergeben sich aus den bestimmten kommunikativen Absichten des Parteiprogramms und stellen die Mikroverfahren dieses Sprachspiels dar. Aus der Zielbestrebung im Bereich der öffentlich-politischen Außenkommunikation resultiert das Makroverfahren, den Bürger/Wähler zu AKTIVIEREN. Das Makroverfahren AKTIVIEREN lässt sich wiederum untergliedern nach (i) ÜBERZEUGEN (daraus ergibt sich der Untersuchungsschwerpunkt der Argumentation, da der Bürger/Wähler mit Argumenten überzeugt werden soll), nach (ii) ÜBERREDEN (daraus ergibt sich das Kapitel, das die Behauptungen untersucht) sowie nach (iii) WERBEN (die Kapitel Bekenntnisse und Versprechen stellen die Mikroverfahren dar, mit denen ein Parteiprogramm wirbt). Die Profilfunktion verwirklicht eine Partei durch den Verweis auf Geleistetes (Kapitel Anspruch auf Erfüllbarkeit) sowie durch die Verwendung von Schlagwörtern; das sprachliche Handlungsmuster könnte als parteiliches ETIKETTIEREN bezeichnet werden.

Ebenfalls im Bereich der öffentlich-politischen Außenkommunikation, allerdings mit doppeltem Adressatenbezug (der Text ist nicht nur auf die zu aktivierende Öffentlichkeit, sondern auch direkt an die politischen Akteure, d.h. Parteiangehörige, gerichtet), werden die Makroverfahren SOLIDARISIEREN und ORIENTIEREN realisiert; dies geschieht durch die Verwendung von Appellen und Aufforderungen (als Anweisung zum praktischen politischen Handeln), aber auch durch Bekenntnisse (durch eine gemeinsame weltanschauliche Grundlage wird die Orientierungsfunktion verbindlich erfüllt). Daher wirkt ein Parteiprogramm in zweifachem Sinne orientierend:

nach innen als Bündelung der politischen Willensbestrebungen in einer Richtung und damit als Appell an die Mitglieder, und nach außen

152 Eine solche Untersuchung beruht maßgeblich auf dem Verständnis des Analysierenden; dieses gewinnt er durch Interpretation der Sequenzen der Dokumente. Die Analyse der Textstrukturen, besonders hinsichtlich der Kapitel Präsuppositionen (2.1.2), Argumentationen (2.1.3) und Behauptungen (2.1.4), können daher keinen Objektivitätsanspruch erheben, da die Beschreibung subjektiven Eindrücken folgt. Diese sollen jedoch so anschaulich und nachvollziehbar wie möglich dargelegt werden, um ein hohes Maß an Plausibilität zu erreichen. Nach Möglichkeit sollen mehrere Interpretationsmöglichkeiten dargeboten werden. 
als positive Selbstdarstellung der Partei und damit als impliziter Appell, der Partei gegenüber eine positive Haltung einzunehmen. ${ }^{153}$

Die Kapitel Expliziter Adressatenbezug und Impliziter Adressatenbezug stellen das Makroverfahren INTEGRIEREN dar (so ist die Integration möglichst aller Parteiflügel durch Kompromiss- und Konsensbildung ein wesentlicher Zweck des Parteiprogramms), und aus den Makroverfahren POLEMISIEREN und DELEGITIMIEREN (dem politischen Gegner soll die Legitimität abgesprochen werden) resultieren die Untersuchungsschwerpunkte der Anklagen und Vorwürfe sowie das Kapitel Expliziter Gegnerbezug. Das Makroverfahren LEGITIMIEREN findet seine Entsprechung im Kapitel Das Godesberger Programm und der GrundgesetztextGemeinsamkeiten und Unterschiede, da ein großer Teil der Forderungen nach der Vorlage eines Gesetzestextes konzipiert sind und dadurch legitimiert wird. In den Bereich der Innenkommunikation (oder Binnenkommunikation) fällt das Makroverfahren STABILISIEREN (aufgrund der Handlungsrichtlinien stabilisiert das Parteiprogramm die Parteibasis).

Als Einstieg in die pragmatische Analyse des Godesberger Programms sollen die letzten vier Zeilen der Präambel herangezogen werden (vgl. Kapitel 2.1.4.4.1, Zeilen 33-36):

(3) (a) Nur durch eine neue und bessere Ordnung der Gesellschaft öffnet der Mensch den Weg in seine Freiheit.

(b) Diese neue und bessere Ordnung erstrebt der demokratische Sozialismus.

Die beiden Äußerungen (a) und (b) sind offensichtlich als Mischung von Selbstdarstellung der SPD, von Wahlversprechen oder Versprechen allgemein und von Wählerwerbung zu verstehen. Allerdings bergen die beiden Äußerungen Absichten und Aussagen in sich, die nicht offensichtlich, dennoch aber vorhanden sind;

in Argumentationen kommt es vor, daß mehr verstanden wird, als der betreffende Sprecher zum Ausdruck bringen wollte; es entsteht dann das schwierige Problem, ob der Sprecher aufrichtig ist und bestimmte Verständnisse tatsächlich nicht intendiert, oder ob dies gerade eine Strategie ist $[\ldots] .{ }^{154}$

Daraus ergibt sich nun die zu überprüfende Vermutung, dass es bei der politischen Sprachverwendung folglich auch um solche Aussagen geht, die vom Rezipienten mitgedacht werden, obwohl sie nicht explizit formuliert sind, und wie diese mitgedachten Inhalte in einen Bezug zur möglichen Intention des Sprechers zu setzen sind.

153 Klein 1991a, S. 273

154 Kienpointner, Manfred: Argumentationsanalyse. Innsbruck 1983, S. 33 


\subsubsection{Präsuppositionen}

So wird bei der Konzeption solcher Formulierungen im politischen $\mathrm{Zu}-$ sammenhang davon ausgegangen, dass bei den Sprachteilhabern gleiche Vorannahmen ausgelöst werden, durch die bestimmte Teile der Satzkonstruktion hinsichtlich der erstrebten Textaussage überhaupt erst sinnhaft oder gehaltvoll erscheinen. Solche Vorannahmen werden als Präsuppositionen bezeichnet:

Mit dem Konzept der Präsuppositionen wird unter anderem versucht, die Funktion von aussersprachlichen Wissensbeständen bei der Konstitution von Textkohärenz zu erfassen und zu erklären. ${ }^{155}$

Unter dem Begriff der Textkohärenz werden die "grundlegenden allgemeinen Bedingungen gefaßt, die ein sprachliches Gebilde erfüllen muß, um überhaupt als Text zu gelten, die also für seine ,Textualität' (Texthaftigkeit) konstitutiv sind. “156 Der Aspekt der Textkohärenz kann bei den hier zugrunde liegenden Beispielsätzen der Präambel vernachlässigt werden, da die Sätze keine Ergänzungen auf der linguistischen Ebene erfordern, um verständlich oder nachvollziehbar im Sinne der Textkohärenz zu sein. Wichtiger ist die Vermittlung der jeweiligen Inhalte. Präsuppositionen sind Propositionen, die über den spezifischen Wahrheitsanspruch hinaus ein mitgedachtes Weltwissen erfordern, das in den Sätzen nicht explizit formuliert wird. Daher sind unter diesem kollektiven Wissen

alle Propositionen zu verstehen, von denen alle Gesprächsteilnehmer ehrlich behaupten können, zu wissen, daß sie wahr sind. Zusätzlich wissen sie, daß der jeweilige Partner ehrlich von sich behaupten kann zu wissen, daß sie wahr sind. ${ }^{157}$

Unter Präsuppositionen werden folglich mitgemeinte bzw. unausdrücklich mitbehauptete Propositionen (Vor- oder Grundannahmen) verstanden, deren Wahrheit erst das Gelingen von Sprechakten bestimmter Art ermöglicht (präsuppositionale Akte); sie werden daher von STALNAKER (1972) mit der folgenden Ausführung definiert:

Presuppositions are probably best viewed as complex dispositions which are manifested in linguistic behaviour. One has presuppositions in virtue of the statements he makes, the questions he asks, the

\footnotetext{
155 Linke/Nussbaumer/Portmann 1996, S. 231

156 Brinker, Klaus: „Aspekte der Textlinguistik.“ In: Germanistische Linguistik (106-107). Hg. v. Klaus Brinker. Hildesheim/Zürich/New York 1991, S. 13

157 Kienpointner 1983, S. 61
} 
commands he issues. Presuppositions are propositions implicitly supposed before the relevant linguistic business is transacted." 158

Bei den Formulierungen nur durch eine neue und bessere Ordnung (a) und diese neue und bessere Ordnung erstrebt (b) legt sich der Sprecher konventionell auf bestimmte Prämissen fest in der Annahme, dass der Rezipient diese impliziten Annahmen auf die gleiche Weise zur Kenntnis nimmt. Der Sprecher präsupponiert folgende Wissensvoraussetzungen oder Grundannahmen:

1. Prämisse P1: Derzeit existiert eine Ordnung, die weniger tauglich oder gut als die erstrebte ist;

2. Prämisse P2: Freiheit kann mit der bestehenden Gesellschaftsordnung nicht erreicht werden;

3. Prämisse P3: Es gibt ein Bedürfnis und ein Recht der Bevölkerung auf eine bessere Ordnung im Gegensatz zur schlechteren, die derzeit existiert;

4. Prämisse P4: Die SPD als die diese Aussagen treffende Partei ist in der Lage und willens, die bessere Ordnung zu verwirklichen;

5. Prämisse P5: Dabei hat die SPD das Wohl der Bevölkerung im Auge und alles geschieht zum Zwecke einer Verbesserung der derzeitigen gesellschaftlichen Verhältnisse;

6. Prämisse P6: Als höchstes Ziel sieht die SPD die Freiheit an; diese ist momentan noch nicht erreicht und kann auch nur mit der SPD erreicht werden.

Auf der Basis dieser Prämissen ergibt sich letztlich die folgende und beabsichtigte Schlussfolgerung: Jeder Bürger, der ebenso wie die SPD eine bessere Ordnung als die derzeitige sowie Freiheit erstrebt, sollte sich zur SPD bekennen und seine Stimme bei einer Wahl dieser Partei geben, um deren Position zur Erreichung der formulierten Ziele zu stärken. Dieses Grundmuster ist nicht auf das Godesberger Programm beschränkt, da die anderen Parteiprogramme ebenso diesen Anspruch erheben; so formuliert die SPD 30 Jahre nach dem Godesberger Programm folgende Aussage: „Eine neue und bessere Ordnung, der Würde des Menschen verpflichtet, ist daher möglich und nötig zugleich" (SPD 1989, S. 11).

Eine besondere Bedeutung kommt dem verwendeten Verb erstreben $\mathrm{zu}$, da durch dessen Gebrauch deutlich wird, dass der angestrebte Zustand zum Zeitpunkt der Formulierung noch nicht realisiert wurde. Dadurch handelt es sich bei der dargestellten Ausführung hinsichtlich des Wortes erstreben um einen Typus von Präsuppositionen, der an den materiell gegebenen Text, d.h. an die Semantik einzelner Wörter oder Ausdrücke gebunden ist (Semantische Präsupposition); „es handelt sich hier um eine

158 Stalnaker, Robert C.: „Pragmatics.“ In: Semantics of Natural Language. Hg. v. Donald Davidson und Gilbert Harman. Dordrecht 1972, S. 387/388 
Art nicht direkt angesprochener, aber eben mitgemeinter Bedeutung. “159 Dieser Typus ist neben der Referentiellen Präsupposition (auch Existenzpräsupposition) Teil von Zeichengebundenen Präsuppositionen; diese erlauben es Sprechern,

mit der Äusserung bestimmter Sätze gewisse Tatbestände und Sachverhalte mitzubehaupten bzw. als gegeben zu unterstellen, die selbst nicht explizit thematisiert bzw. mit den verwendeten Ausdrücken nicht explizit , bedeutet' sind. ${ }^{160}$

Eine weitere Form solcher Textverständnisvoraussetzungen sind die Gebrauchsgebundenen Präsuppositionen (auch Pragmatische Präsuppositionen). Letztere sind nicht sprachlich formulierte Wissensbestände oder Alltagserfahrungen, die aber durch den Text vorausgesetzt und in den meisten Fällen problemlos mitverstanden werden. Sie werden als gebrauchsgebunden oder pragmatisch bezeichnet,

weil diese Präsuppositionen einem sprachlichen Ausdruck nicht grundsätzlich anhaften, sondern sich erst aus dem Gebrauch, den man von einem sprachlichen Ausdruck macht, ergeben. ${ }^{161}$

Die pragmatische Präsupposition in der oben dargestellten Ausführung resultiert aus der Kombination des Adjektivs neu mit dem Komparativ besser bzw. aus der Konstruktion neue und bessere Ordnung. Wenn man allgemein etwas fordert, was besser ist, dann ist es nicht unbedingt notwendig, dasjenige zu erwähnen, was durch das bessere oder neue ersetzt oder verbessert werden soll; man setzt das Wissen voraus, dass das derzeitige nicht so gut ist wie das geforderte. Beim Rezipienten muss daher ein nicht im Text realisierter gedanklicher Zwischenschritt erfolgen, um die Formulierungen eines Textes in einen sinnvollen Zusammenhang zu bringen:

Gebrausgebundene Präsuppositionen sind also diejenigen Voraussetzungen, die Sprecher und Sprecherinnen als gegeben setzen, wenn sie eine Äußerung (einen Satz, einen Text) in einer konkreten Situation kommunikativ sinnvoll verwenden. ${ }^{162}$

Alle Typen von Präsuppositionen setzen folglich ein bestimmtes Mitwissen als gegeben voraus, wenn Äußerungen als sprachliche Handlungen vollzogen werden. Diese Feststellung ist insofern interessant, als dass die SPD danach vermutlich voraussetzt, dass die Rezipienten die oben dargestellten Prämissen P1 bis P6 durch die Formulierungen des Parteiprogramms genau auf diese Weise wahrnehmen; die letzten vier Sätze der

$\begin{array}{ll}159 & \text { Linke/Nussbaumer/Portmann 1996, S. } 232 \\ 160 & \text { Linke/Nussbaumer/Portmann 1996, S. } 233 \\ 161 & \text { Linke/Nussbaumer/Portmann 1996, S. } 232 \\ 162 & \text { Linke/Nussbaumer/Portmann 1996, S. } 232\end{array}$ 
Präambel werden so in einen sinnvollen Zusammenhang gebracht, ohne dass dieser zuvor explizit formuliert werden musste.

Die unterschiedlichen Typen von Präsuppositionen lassen sich nach folgendem Schema anordnen:

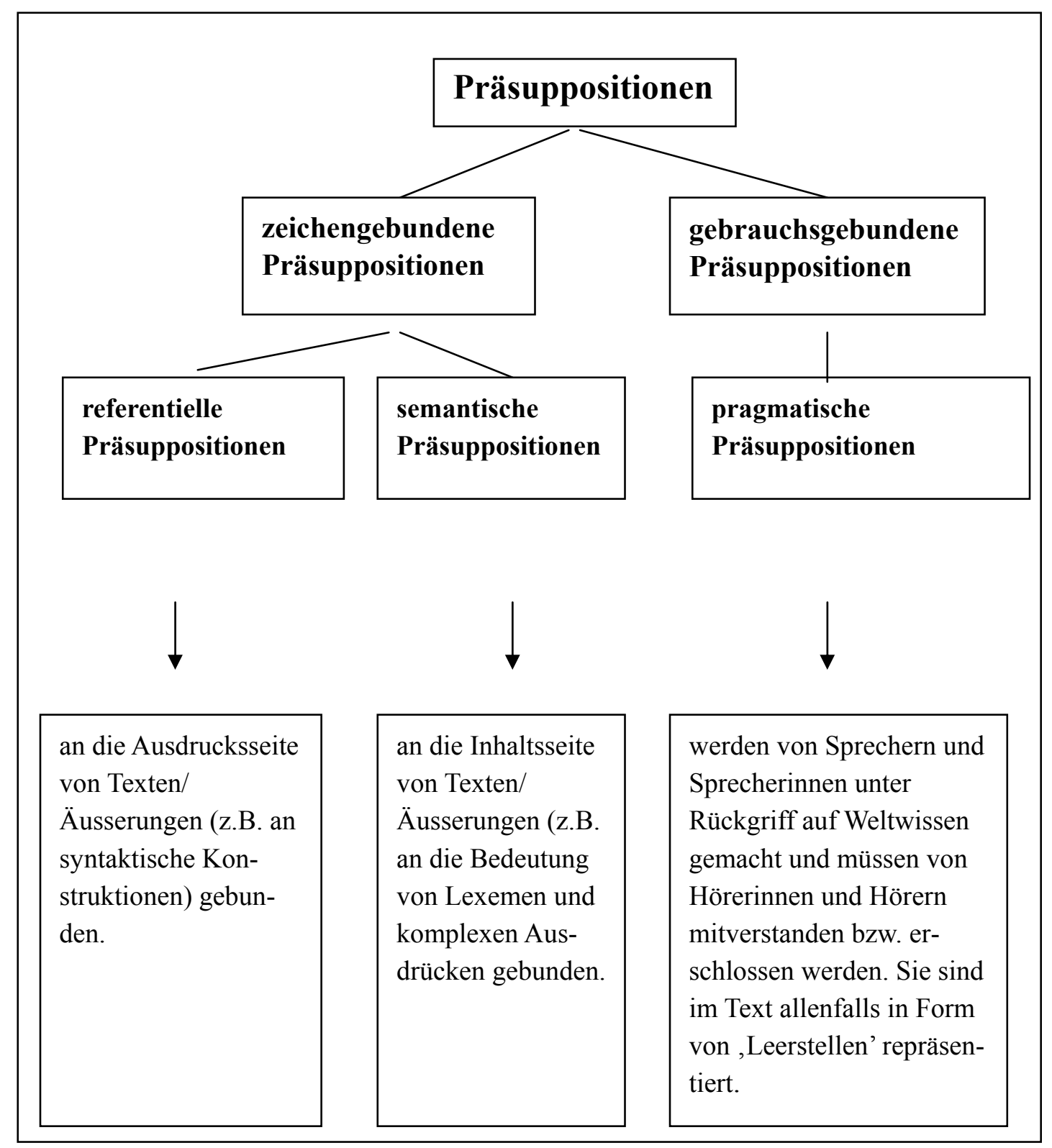

Abbildung 5: (vgl. LinKe/NusSBAumER/PORTMANN 1996, S. 234)

Als Fazit lässt sich abschließend festhalten: Die dargestellte Betrachtung der Präsuppositionen belegt, dass außersprachliche bzw. implizite Verstehensgrundlagen existieren, die zwar einerseits in gewissen Kontexten zu Missverständnissen führen können, die andererseits aber auch als Ursache für Beeinflussungen oder Überzeugungen anzusehen sind, abhängig von der Sprechsituation sowie der Kooperationsbereitschaft und dem Weltwissen des Rezipienten. Aufgrund von Präsuppositionen weiß der Rezipient über den Sprecher, welche Einstellungen und Ansichten dieser hat, ohne dass diese explizit formuliert wurden. 
Allerdings kann auch mit expliziten sprachlichen Handlungen überzeugt werden, indem im Zuge einer Argumentation Argumente dargelegt oder erörtert werden.

\subsubsection{Argumentationen}

Politische Texte sind normalerweise nicht allein beschreibend, appellativ und/oder diffamierend, sondern sie sind vor allem argumentativ [...]. Wer politische Äußerungen macht, der vertritt in aller Regel bestimmte Thesen, die er des weiteren zu begründen oder zu belegen sucht, und er zieht Schlussfolgerungen. ${ }^{163}$

Die Untersuchung der argumentativen Strukturen des Godesberger Programms versucht aufzuzeigen, wie bestimmte Thesen der SPD durch die Verwendung von Argumenten gestützt und möglicherweise Aussagen des politischen Gegners durch Argumente entkräftet werden. Grundlage dieser Teilanalyse ist folglich ein schriftlich fixierter Text; diese Tatsache wirkt sich auf die Art einer möglichen Argumentation insofern aus, als dass diese sich nicht zwischen direkten Interaktionspartnern ereignet (wie beispielsweise in einer mündlichen Diskussion oder einem Briefwechsel im schriftlichen Bereich), sondern lediglich auf die Argumente der SPD innerhalb ihres Parteiprogramms bezieht. Eine mögliche Argumentation ist daher in das Parteiprogramm eingebettet.

Was ist nun grundsätzlich unter den Ausdrücken Argument und Argumentation zu verstehen? Ein notwendiger Exkurs in die komplexe Theorie des Argumentationsprinzips soll die Fragestellung zu klären helfen.

Die Argumentation ist „ein kommunikativ manifestiertes Verfahren, das dazu dient, zu Entscheidungen über die Geltung von Aussagen zu gelangen." 164

Das Argumentieren als sprachliche Handlung ist unmittelbar an die Absicht geknüpft, den Rezipienten oder allgemein den Empfänger von einer These zu überzeugen:

Die meisten argumentierenden Texte sind sprachlich so aufgebaut, daß sich aus der (ideologisch geprägten) Darstellung der Situation eine Lagebewertung ergibt, die die Intentionen des Redners geradezu von selbst rechtfertigt. ${ }^{165}$

Die Intention des Redners/Schreibers besteht in dessen Versuch, den Rezipienten dahin zu bewegen, seine Beurteilung der Lage zu überneh-

\footnotetext{
163 Burkhardt 1988, S. 353

164 Kindt, Walther: „Argumentationskultur in Bundestagsreden - illustriert am Bespiel einer Debatte vom 2.4.1998.“ In: Sprache des deutschen Parlamentarismus. Hg. v. Armin Burkhardt/Kornelia Pape. Wiesbaden 2000, S. 321

Bachem 1979, S. 93
} 
men. Man könnte also sagen, dass das Anbringen eines Arguments aufgrund der Begründung in weit stärkerem Maße motiviert ist als die Formulierung einer Behauptung, wenn die Behauptung als bloße Mitteilung oder Kundgebung der Ansicht des Sprechers/Schreibers gewertet wird; die Begründung dient hierbei „der Stützung und Problematisierung der Entkräftung des Geltungspostulats für Aussagen. ${ }^{\prime 166}$

Das Aufstellen einer zwischen den verschiedenen Kommunikationspartnern umstrittenen Behauptung ist allerdings als Voraussetzung für ein Argument, das erst durch weitere Informationen und Behauptungen, die das Umstrittene stützen bzw. dessen Falschheit zeigen sollen, zu diesem wird. Das Argument wiederum steht dann in direktem Bezug zur vorher formulierten Behauptung. Folglich kann eine Behauptung dann nachgewiesen werden, „wenn der Sprecher [...] für eine Äußerung nicht von vornherein den Anspruch auf Unstrittigkeit stellen kann, wenn also der Sprecher eine Problematisierung des von ihm gestellten Wahrheitsanspruchs nicht ausschließen kann.. ${ }^{167}$

Eine Argumentation besteht daher aus vielen Behauptungen, die unter dem Aspekt der Folgerichtigkeit aneinandergereiht sind und bestimmte Aussagen begründen; argumentationstypische sprachliche Markierungen können dabei als Indikatoren für eine Argumentation oder die "Argument-Konklusions-Beziehung "168 gelten (wie beispielsweise die in Begründungen verwendete Konjunktion weil sowie deshalb, wegen, aufgrund von, denn, da, zumal, deswegen, daher, darum, um zu und weil...will/möchte, sowie weiterhin einige konzessive und adversative Indikatoren wie trotz, trotzdem, obwohl, obgleich, obschon, wenn auch, wenn schon, nichtsdestoweniger, gleichwohl, dennoch, doch oder insofern). Als Kriterium für ein Argument lässt sich somit festhalten,

daß eine Behauptung nur dann ein Argument für eine andere Behauptung sein kann, wenn zwischen dem Argument und der zu stützenden Behauptung, die im weiteren Verlauf Konklusion genannt werden soll, eine Folgerungsbeziehung besteht. Anders ausgedrückt: Ein Satz p kann nur dann als Argument für q verwendet werden, wenn $q$ in irgendeiner Weise aus $p$ folgt. ${ }^{169}$

166 Kindt 2000, S. 321

167 Klein, Josef: Die konklusiven Sprechhandlungen. Studien zur Pragmatik, SEMANTIK, Syntax und Lexik von BEGRÜNDEN, ERKLÄREN-WARUM, FOLGERN und RECHTFERTIGEN. Tübingen 1987, S. 32

168 Klein 1987, S. 10

169 Öhlschläger, Günther: „Argumentieren.“ In: Einführung in die Praktische Semantik. Hg. v. Hans Jürgen Heringer/Günther Öhlschläger/Bruno Strecker/Rainer Wimmer. Heidelberg 1977, S. 256 
Die Folgerichtigkeit aus der Konklusion, dem Argument der vorausgesetzten Wahrheit (Prämisse) zusammen können als Argumentation betrachtet werden. Dabei ist

die Konklusion das Strittige, also der Teil, um dessen Wahrheit es geht, das Argument der Teil, mit dessen Hilfe die Wahrheit bzw. die Falschheit des Strittigen gestützt werden soll, und die Schlußpräsupposition der Teil, der den Übergang vom Argument zur Konklusion rechtfertigt, d.h. der Teil, aufgrund dessen die als Argument angeführte Behauptung überhaupt erst ein Argument für die Konklusion sein kann. ${ }^{170}$

Diesen Ausführungen zufolge ergibt sich folgendes Schema:

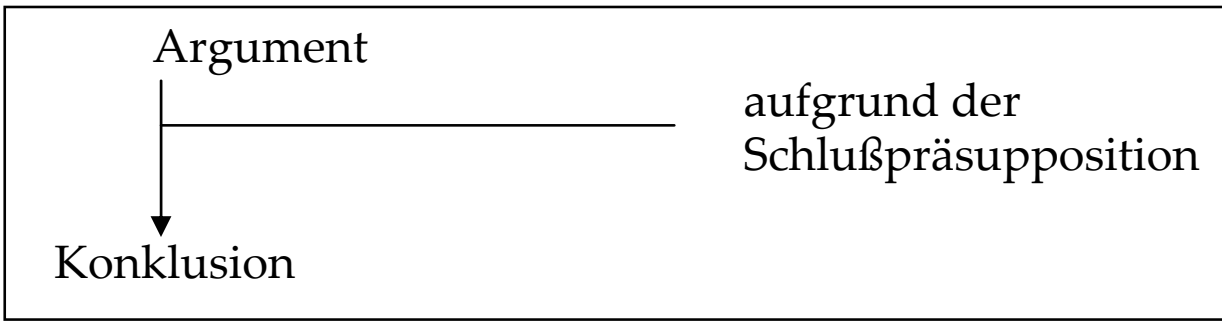

Abbildung 6: (vgl. ÖHLSCHLÄGER 1977, S. 262)

In oben angeführtem Zitat wird der Ausdruck Prämisse durch den der Schlusspräsupposition ersetzt. Tatsächlich ist die Verwendung des Ausdrucks Prämisse (oder präziser Unter- und Oberprämisse sinngemäß für Argument und Schlusspräsupposition) in diesem Zusammenhang nicht unproblematisch, da dessen Verwendung nicht spezifisch für das Argumentieren, sondern für alle Schlüsse und Bereiche der Logik anwendbar ist. ${ }^{171} \mathrm{Im}$ Folgenden wird daher auch in dieser Untersuchung von (Schluss-)Präsuppositionen anstelle von Prämissen die Rede sein.

Da sich die bisherigen Ausführungen auf die Theorie der Argumentation beschränkten, soll im Folgenden versucht werden, die Prinzipien an

170 Öhlschläger 1977, S. 262

171 Ähnlich unklar ist ebenfalls der verwendete Ausdruck des Arguments; dieser wird oftmals in der Weise verwendet, dass die Kombination bzw. die Schlussfigur aus Argument, Konklusion und Schlusspräsupposition unabhängig von pragmatischen Aspekten als Argument bezeichnet wird. Der Ausdruck wird dadurch mehrdeutig, weshalb die Verwendung des Ausdrucks Argumentation als Benennung vorgezogen wird, allerdings wird auch dieser Terminus häufig eingeschränkt auf solche Sprechhandlungen, die die Merkmale Schlusscharakter und Strittigkeit aufweisen. Danach sind alle Argumentationen stets als Begründungen für strittige Einstellungen zu werten, wobei jedoch nicht alle Begründungen auch Argumentationen darstellen. Die Bezeichnung Argumentation kann dann weiterhin in das Argumentationsergebnis und in die Argumentationshandlung differenziert werden. 
einer konkreten Textpassage des Godesberger Programms aufzuzeigen. Es bietet sich hierfür folgende Textstelle an:

(4) (a) Freiheit und Gerechtigkeit lassen sich durch Institutionen allein nicht sichern.

(b) Alle Lebensbereiche werden zunehmend technisiert und organisiert.

(c) Dadurch entstehen immer neue Abhängigkeiten, die die Freiheit bedrohen.

(d) Nur ein vielgestaltiges wirtschaftliches, soziales und kulturelles Leben regt die schöpferischen Kräfte des einzelnen an, ohne die alles geistige Leben erstarrt.

(e) Freiheit und Demokratie in der industriellen Gesellschaft sind nur denkbar, wenn eine ständig wachsende Zahl von Menschen ein gesellschaftliches Bewußtsein entwickelt und zur Mitverantwortung bereit ist.

(f) Ein entscheidendes Mittel dazu ist politische Bildung im weitesten Sinne.

(g) Sie ist ein wesentliches Ziel aller Erziehung in unserer Zeit. (Godesberger Programm 1959, S. 70/71)

Die zitierten Beispielsätze (a) bis (g) sind im Originaltext als zusammenhängende Textpassage abgedruckt; (d) und (e) sind durch einen Absatz unterteilt. Die Argumentation beginnt mit (a) als einem strittigen Teil, um dessen Wahrheit es geht (Konklusion), nämlich dass Freiheit und Gerechtigkeit als Grundwerte nicht durch Institutionen allein gesichert werden können. Nun folgt eine Begründungs- oder Herleitungskette aus impliziten Schlusspräsuppositionen und expliziten Feststellungen und Behauptungen. Diese Kette beginnt mit der Feststellung (b), dass die Lebensbereiche zunehmend technisiert und organisiert werden; dabei leitet die Feststellung (b) die Behauptung (c) ein, die besagt, dass dadurch neue, die Freiheit bedrohenden Abhängigkeiten entstehen. Die Sätze (b) und (c) müssen zusammen betrachtet werden, da zwischen beiden eine inhaltliche bzw. sinngemäße Folgerichtigkeit besteht, die darüber hinaus formal durch das Wort dadurch gekennzeichnet wird; (c) resultiert aus (b) bzw. (c) ist als eine konkrete Auswirkung bzw. Erscheinung von (b) zu betrachten. Interessanterweise wird im zweiten Teil der Äußerung von (c) eine der Schlusspräsuppositionen von (a) formuliert:

1. Schlusspräsupposition S1: Freiheit und Gerechtigkeit sind niemals als sicher sondern als gefährdet und bedroht zu betrachten und müssen daher gesichert werden;

2. Schlusspräsupposition S2: Freiheit und Gerechtigkeit können mit anderen Mitteln als durch Institutionen gesichert werden. 
Die Feststellung (b) und die Behauptung (c) sind deswegen Argumente, da mit deren Hilfe (durch die Begründungen, die sie liefern) die Wahrheit von (a) als strittige Ausgangsthese (Konklusion) gestützt wird; dies geschieht aufgrund der formulierten Schlusspräsuppositionen S1 und S2 als Teile, die die Entwicklung der Behauptung zum Argument für die Konklusion überhaupt erst ermöglichen. Diese Ansicht kann jedoch aufgrund individueller Rezeption differenziert interpretiert werden: Es ist ebenso möglich, bereits Äußerung (b) als Behauptung zu betrachten; folglich wird diese durch (c) als Zusatzbehauptung zum Argument. Die Äußerung (d) stellt streng genommen eine erneute Konklusion dar, da auch dieser Teil der Argumentationskette als strittig anzusehen ist; jedoch ist (d) als Forderung zu verstehen, die als Konsequenz aus der Herleitung von (a), (b) und (c) resultiert. Nur die Kreativität des Individuums in der Gesellschaft wirkt den Abhängigkeiten entgegen, die die Freiheit bedrohen, und trägt entscheidend zu einem kulturellen Leben bei. Wie kann nun dieses freie und gestalterische Leben in der Gesellschaft erreicht werden? Diese Frage beantwortet die Behauptung (e). Die Aussage ist zwar wieder strittig und könnte daher von diesem Standpunkt aus als Konklusion betrachtet werden. Es fehlen jedoch das Argument und die Schlusspräsupposition; die Argumentation der Äußerungen (e) bis (g) präsentiert sich daher grundlegend anders. Es ist genau genommen auch nicht von Argumentation zu sprechen, da keine Begründungen als „Stützen des Wahrheitsanspruchs für den Inhalt einer Behauptung "172, sondern lediglich eine erneute Forderung (f) sowie die Formulierung der Zielbestrebung (g) geliefert werden. Die Forderung nach politischer Bildung als wichtigem Beitrag zur Freiheit und Demokratie in der Gesellschaft ist keine Begründung, sondern eine Präzisierung. Die Äußerungen (a) bis (g) bleiben zwar kohärent (nicht zuletzt das Wort dazu in (f) trägt als semantisch-syntaktische Verknüpfung an der Textoberfläche zur inhaltlichen Anknüpfung bei), um jedoch (e) bis (g) als Argumentation zu werten fehlt eine Begründung wie (c).

Die Argumentation der Äußerungen (a) bis (g) können aber auch auf eine Weise interpretiert werden, die sich von der bereits dargestellten unterscheidet. Es ist möglich, die Thesen (a) und (f) zum Ausgangspunkt der Interpretation zu machen; (a) als Konklusion bleibt dabei bestehen. Als strittiger Teil wird (a) durch (e) als Argument hinsichtlich des Wahrheitsanspruchs gestützt. Inhaltlich bedeutet diese Feststellung, dass das gesellschaftliche Bewusstsein und die Mitverantwortung Freiheit und Gerechtigkeit sichern, was Institutionen allein nicht erreichen. Die Beziehung zwischen Konklusion (a) und Argument (e) wird durch zwei Seiten gestützt: Einerseits wird sie gestützt durch die Forderung (f) und die Formulierung der Zielbestrebung (g), die nochmals die Bedeutung

172 Klein 1987, S. 21 
der Notwendigkeit politischer Bildung betonen und damit das Kapitel Grundforderungen für eine menschenwürdige Gesellschaft abschließen; andererseits durch eine eigenständige Argumentation, bestehend aus der Konklusion (d), dem Argument (b) sowie der explizit formulierten Schlusspräsupposition (c). Im Unterschied zur ersten Interpretation ergeben sich die Schlusspräsupposition nicht aus dem Mitgedachten der Äußerungen, sondern werden durch die expliziten Begründungen des Textes verkörpert. Diese Argumentation wird "Argumentationskette" oder "gestufte Argumentation" dargestellte Schaubild 6 von ÖHLSCHLÄGER (1977) ergibt sich für diese Lesart der Argumentation nun folgendes Schema:

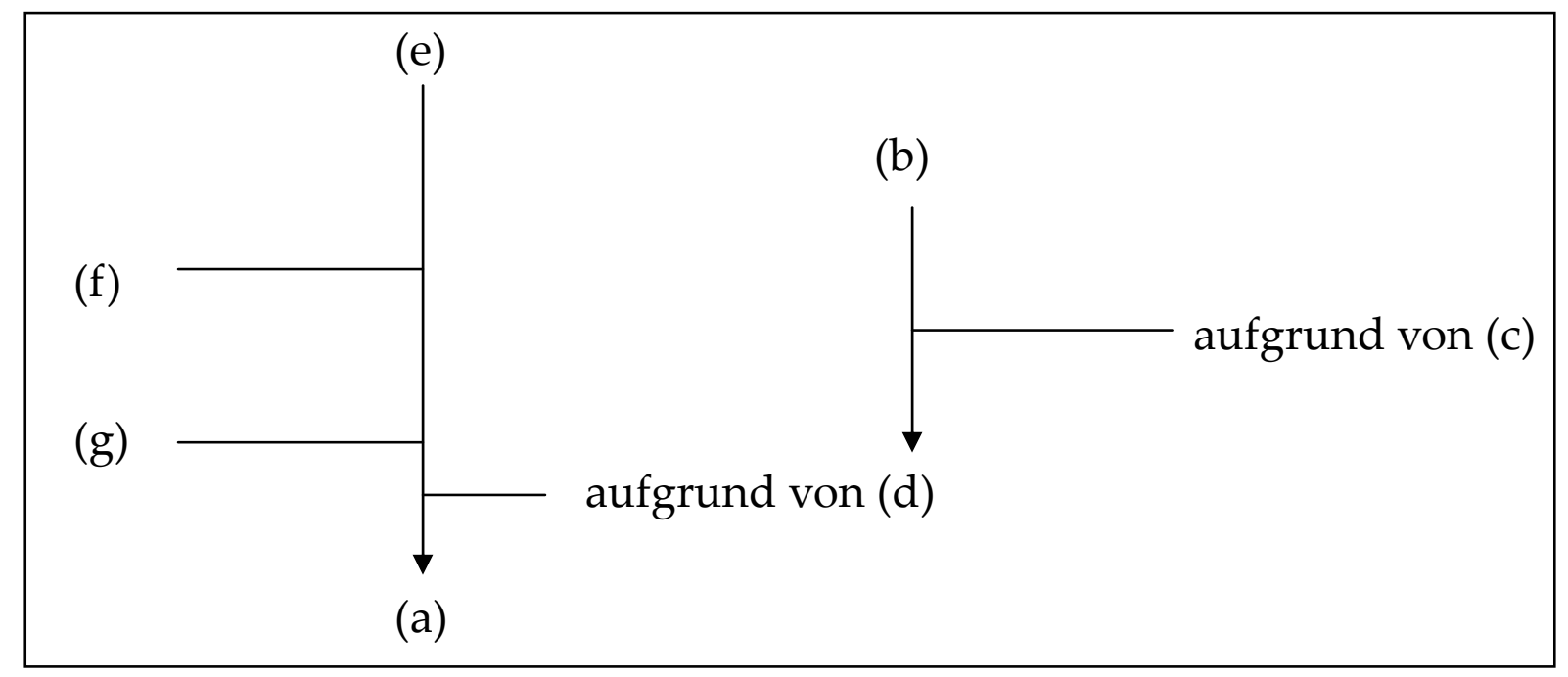

\subsubsection{Behauptungen}

Wie oben bereits erwähnt geht die Formulierung einer Behauptung einer Argumentation voraus bzw. ist im Rahmen einer Begründung Bestandteil einer Argumentation (Behauptungen werden in diesem Fall als Argumente zur Stützung eines strittigen Geltungsanspruchs gebraucht); „Behauptunygen werden im Deutschen u.a. durch Indikativsätze mit Zweitstellung des Prädikats realisiert [...].“174 Eine Behauptung scheint sich von einem Argument sowohl im Hinblick auf die Folgerichtigkeit als auch in Bezug auf das Überzeugungspotential zu unterscheiden; daher kann die Behauptung als bloße Meinungskundgebung verstanden werden. Eine Behauptung ist jedoch - wie die Argumentation - an eine Präsupposition geknüpft; diese Annahme scheint gerechtfertigt zu sein, "weil es der Sinn von Behauptungen ist, einen Wahrheitsanspruch für das Behauptete aufzustellen, d.h. etwas zu sagen, das nach Wahrheit und 
Falschheit beurteilbar ist." 175 Mit der Sprechhandlung BEHAUPTEN wird folglich ein Wahrheitsanspruch erhoben. Die darauf Bezug nehmenden Sprechhandlungen der Kommunikationspartner (BESTREITEN, ENTGEGNEN, ZUSTIMMEN) richten sich nicht auf die illokutionäre Rolle des BEHAUPTENS, sondern auf den propositionalen Gehalt (es geht daher um die Inhalte der Behauptung).

Der nachfolgend zitierte Textauszug soll als Beispiel für Behauptungen herangezogen werden:

(5) (a) [...] In seinem Sinne erstrebt sie [die SPD] die Einheit Deutschlands in gesicherter Freiheit.

(b) Die Spaltung Deutschlands bedroht den Frieden.

(c) Ihre Überwindung ist lebensnotwendig für das deutsche Volk.

(d) Erst in einem wiedervereinigten Deutschland wird das ganze Volk in freier Selbstbestimmung Inhalt und Form von Staat und Gesellschaft gestalten können. (Godesberger Programm 1959, S. 71)

Mit Hilfe der Äußerung (a) wird zunächst eine Zielbestrebung der SPD formuliert; diese Aussage wird durch das Verb erstreben markiert und kann auch als Konklusion betrachtet werden (es geht im Folgenden um die Wahrheit dieser These). Nun folgt mit der Äußerung (b) die Behauptung, dass die Freiheit durch die Spaltung Deutschlands bedroht sei. Zwar ist (b) als Begründung für das zuvor formulierte Ziel in (a) zu verstehen, die Behauptung (b) wird jedoch nicht gestützt. Die Aussage (c), dass die Überwindung der Spaltung lebensnotwendig sei für das deutsche Volk mag stimmen; die Aussage begründet aber (b) nicht, sondern betont diese und bleibt als abstrakte Behauptung ihrerseits bestehen. Auch Aussage (d) begründet zwar in gewisser Weise die Notwendigkeit der Wiedervereinigung. Als Begründung kann die Aussage dennoch nicht gewertet werden, da die jeweiligen Themen Selbstbestimmung sowie Gestaltung von Inhalt und Form von Staat und Gesellschaft nicht präzisiert werden und als abstrakte Punkte in globaler Verwendung erscheinen. Da nicht explizit ausgeführt wird, inwiefern sich die Mitbestimmung und Gestaltung im Detail entwickeln, kann (d) somit nur als Weiterführung der vorangegangenen Behauptungen betrachtet werden; Äußerung (d) stellt als argumentative Behauptung somit eine Sonderform der Behauptungen dar, da durch deren Verwendung die Zielbestrebung in (a) gestützt wird. Zwar kann eine Schlusspräsupposition zu (b) aufgrund des Weltwissens des Rezipienten formuliert werden (Frieden ist ein gefährdetes Gut in der Gesellschaft und muss angestrebt und gesichert werden) und der Aspekt der Folgerichtigkeit zwischen den einzelnen Aussagen (a) bis (d) besteht ebenfalls; dennoch stellt die zitierte Textpas-

175 Öhlschläger 1977, S. 258 
sage dieser Herleitung folgend keine Argumentation oder lediglich bedingt eine Argumentation dar. Vielmehr ist die zitierte Passage als eine Aneinanderreihung von Behauptungen zu betrachten.

\subsubsection{Anspruch auf Erfüllbarkeit}

Bei der Konzeption eines Parteiprogramms muss der Aspekt der Erfüllbarkeit berücksichtigt werden, wenn das Programm den Anspruch zu erheben beabsichtigt, dem potentiellen Wähler ein realistisches, d.h. realisierbares Programm vorzulegen. Der Wähler geht bei seiner Wahl einer Partei davon aus, dass deren formulierten Ziele durchführbar sind. Das Parteiprogramm muss durch die Formulierungen versuchen, den Wähler von der Realisierbarkeit zu überzeugen. Diese Überzeugung kann mit Hilfe der beiden folgenden Regeln geschehen:

1. „Texte mit uneingeschränkter Programmgeltung gehören in den Programmtext hinein";

2. „Der Beschluß, einen früheren Beschluß in ein Parteiprogramm aufzunehmen, stellt eine Bekräftigung des Beschlusses dar. ${ }^{\prime 176}$

Im Godesberger Programm finden sich keine Belege für die beiden zitierten Regeln; es wird auf kein vorangegangenes Programm hingewiesen. Im SPD-Grundsatzprogramm von 1989 lässt sich dieses taktische Berücksichtigung bei der Konzeption jedoch nachweisen, da hier auf das Heidelberger Programm von 1925 und auf das Godesberger Programm von 1959 hingewiesen wird und die damaligen Inhalte als nach wie vor gültig und verbindlich gelten:

(6) (a) Die Vereinigten Staaten von Europa, von den Sozialdemokraten im Heidelberger Programm 1925 gefordert, bleiben unser Ziel. (SPD 1989, S. 16)

(b) Das Godesberger Programm zog aus den geschichtlichen Erfahrungen neue Konsequenzen. Es verstand Demokratischen Sozialismus als Aufgabe, Freiheit, Gerechtigkeit und Solidarität durch Demokratisierung der Gesellschaft, durch soziale und wirtschaftliche Reform zu verwirklichen. Die Sozialdemokratische Partei stellte sich in Godesberg als das dar, was sie seit langem war: die linke Volkspartei. Sie wird es bleiben. (SPD 1989, S. 8)

Der Anspruch auf Erfüllbarkeit kann daher dadurch deutlich gemacht werden, dass ein Programm auf die Erfüllung früherer Programme oder einzelner Programmpunkte hinweist. Die Erwähnung des Erreichten ist als Erfolgsmeldung zu bewerten und damit eng an die Werbefunktion geknüpft, da die Partei durch den Hinweis auf das Geleistete Tatkraft

176 Klein 1996a, S. 205 
und Leistungsvermögen demonstriert, sich positiv darstellt und dadurch selbst lobt. Im Godesberger Programm findet sich die folgende Textpassage, die einen Exkurs in die Geschichte der Sozialdemokratie darstellt und als Hinweis auf die Erfüllbarkeit der Forderungen interpretiert werden kann. Dem Verweis auf die innerparteiliche Entwicklung in der Geschichte scheint, gemessen am Umfang der Textpassage, ein hoher Stellenwert beigemessen zu werden: Es geht um die Erfüllung einer geschichtlichen Aufgabe:

(7) Die sozialistische Bewegung erfüllt eine geschichtliche Aufgabe. Sie begann als ein natürlicher und sittlicher Protest der Lohnarbeiter gegen das kapitalistische System. Die gewaltige Entfaltung der Produktivkräfte durch Wissenschaft und Technik brachte einer kleinen Schicht Reichtum und Macht, den Lohnarbeitern zunächst nur Not und Elend. Die Vorrechte der herrschenden Klassen zu beseitigen und allen Menschen Freiheit, Gerechtigkeit und Wohlstand zu bringen das war und das ist der Sinn des Sozialismus.

Die Arbeiterschaft war in ihrem Kampf nur auf sich gestellt. Ihr Selbstbewußtsein wurde geweckt durch die Erkenntnis ihrer eigenen Lage, durch den entschlossenen Willen, sie zu verändern, durch die Solidarität in ihren Aktionen und durch die sichtbaren Erfolge ihres Kampfes.

Schweren Rückschlägen und manchen Irrtümern zum Trotz hat die Arbeiterbewegung im neunzehnten und zwanzigsten Jahrhundert die Anerkennung vieler ihrer Forderungen erzwungen. Der einst schutzund rechtlose Proletarier, der sich für einen Hungerlohn täglich sechzehn Stunden schinden mußte, erreichte den gesetzlichen Achtstundentag, den Arbeitsschutz, die Versicherung gegen Arbeitslosigkeit, Krankheit, Siechtum und für seinen Lebensabend. Er erreichte das Verbot der Kinderarbeit, der Nachtarbeit für die Frauen, den Jugendund Mutterschutz und bezahlten Urlaub. Er erstritt sich die Versammlungsfreiheit, das Recht zum gewerkschaftlichen Zusammenschluß, das Tarifrecht und das Streikrecht. Er ist dabei, sein Recht auf Mitbestimmung durchzusetzen. Der einst das bloße Ausbeutungsobjekt der herrschenden Klasse war, nimmt jetzt seinen Platz ein als Staatsbürger mit anerkannten gleichen Rechten und Pflichten. (Godesberger Programm 1959, S. 85/86)

\subsubsection{Versprechen/Zielbestrebungen}

Die Absicht der Partei, mit dem Parteiprogramm zu werben und möglichst viele Adressaten zu erreichen, erweist sich als Problem wenn es darum geht, realistische Absichtserklärungen zu formulieren:

Die Werbekraft verlangt attraktive Ziele, gerade auch was staatliche Leistungen an den Bürger betrifft. So drängt das Streben nach akquisitorischer Wirkung des Programms die Partei in Richtung auf große 
Versprechungen, und zwar an die meisten oder an alle Bevölkerungsgruppen. ${ }^{177}$

Daher sind Versprechen in der politischen Sprachverwendung als (zukunftsorientierte) Sprechakte zu betrachten, „die typischerweise durch Orientierung an Adressatenpräferenzen motiviert sind.“178 Damit der Wähler sich nicht für das eventuell attraktivere Programm einer konkurrierenden Partei entscheidet, müssen ausreichend werbewirksame Zielbestrebungen und Absichtsbekundungen formuliert werden. Eine Möglichkeit hierzu besteht, indem eine Partei einen Sachverhalt oder eine Handlung VERSPRICHT oder GARANTIERT. Als Sprachhandlung ist VERSPRECHEN als (selbst-)verpflichtende Festlegung $\mathrm{zu}$ betrachten

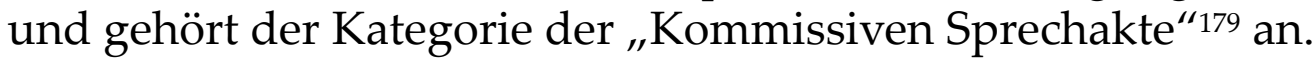

Dabei muss berücksichtigt werden, inwiefern der Wähler die Ziele der Partei für realistisch hält. Meinungsforschungsinstitute können darüber per Wählerumfrage zu einem gewissen Grad Auskunft geben; daher sind sowohl die Demoskopie ${ }^{180}$ als auch der Kontakt zur Parteibasis als wichtige und einflussreiche Faktoren bei der Konzeption eines Parteiprogramms zu bewerten: „Denn Parteien und Politiker, über denen regelmäßig das Damokles-Schwert der Wahlniederlage und der Abwahl hängt, sind aus Gründen des Machterhalts gezwungen, ständig in die Gesellschaft hineinzuhören." 181 Weiterhin muss die Partei selbst wissen, $\mathrm{zu}$ welchen Realisierungen der Versprechen sie überhaupt in der Lage ist (die Partei wird bei Nichterfüllung bzw. Nichteinhaltung der Versprechen nach ihrer Legislaturperiode möglicherweise abgestraft). Daher befindet sich eine Partei bei der Konzeption in einem Dilemma zwischen Realisierbarkeit und Werbewirksamkeit.

In den untersuchten Parteiprogrammen werden Versprechungen auf indirekte Weise formuliert; die geschieht durch die Realisierung kommissiver Sprechakte, jedoch ohne die Verben versprechen oder garantieren explizit zu erwähnen. Der Ausdruck (wir) werden besitzt einen geringeren Festlegungsgrad als versprechen oder garantieren und wird parteiübergreifend mit hoher Frequenz verwendet, daher können nachfolgend

177 Flohr 1968, S. 54

178 
nur einige exemplarische Textstellen angeführt werden. Insbesondere die SED arbeitet bei ihren Formulierungen mit dem Satzmuster werden + Infinitiv (d). Ein Mittel zur Formulierung einer Absichtsbekundung ist weiterhin die Verwendung der Ausdrücke (wir) wollen in den Äußerungen (b) und (c), anstreben in den Äußerungen (e) und (f) und das sehr häufig verwendete Verb erstreben (auch wird mit $(\mathrm{g})$ bis $(\mathrm{k})$ nur eine Auswahl an Beispielen angeführt); insbesondere das Verb erstreben lässt sich in den frühen Parteiprogrammen nachweisen, wie die Äußerungen (j) und (k) zeigen. Auch das Verb beabsichtigen dient zur Bezeichnung einer Absichtsbekundung, es ist an allen untersuchten Parteiprogrammen jedoch lediglich einmal nachweisbar (e):

(8) (a) Wir werden unsere Anstrengungen verstärkt fortsetzen, die Umweltbedingungen zu verbessern. Wir werden den Anspruch aller Menschen auf Reinhaltung von Luft und Wasser, offene Flächen für Freizeit und Erholung und Schutz vor Lärm Geltung verschaffen. [...] Wir werden die Erforschung und Entwicklung abgasfreier Energien und emissionsfreier Maschinen fördern. (CDU 1971, S. 86/87)

(b) Wir wollen eine ausgewogene marktgerechte Struktur von Klein-, Mittel- und Großunternehmen. (CDU 1971, S. 66)

(c) Wir wollen die Ideen, die Kritik und den Protest von Bürgerinnen und Bürgern aufnehmen, sie zu Aktivität ermutigen und ganzheitliche Konzepte entwickeln. (B 90/GRÜNE 2002, S. 10)

(d) Mit dem Sieg des Kommunismus werden die geistige und körperliche Arbeit in der Produktionsbetätigung der Menschen organisch miteinander verschmelzen. Die körperlich Arbeitenden werden kulturell und technisch das Niveau der Geistesschaffenden erreichen. [...] Im Kommunismus werden sich auf der Grundlage der Einheit der gesellschaftlichen und der persönlichen Interessen die Beziehungen zwischen Persönlichkeit und Gesellschaft harmonisch gestalten. (SED 1963, S. 331/332) [...] Die Fähigkeiten und Talente, die besten sittlichen Eigenschaften des freien Menschen werden zur Blüte gelangen und voll zur Entfaltung kommen. Damit werden auch die letzten Überreste der ungleichen Stellung der Frauen im täglichen Leben überwunden. Die Familienbeziehungen werden endgültig frei sein von materieller Berechnung und ganz auf gegenseitiger Liebe und Freundschaft beruhen. (SED 1963, S. 333) [...] Er wird die nationale Gemeinschaft auf eine höhere Stufe heben. [...] Der Sozialismus wird die deutsche Nation durch friedliche Arbeit zur Blüte und Größe führen. (SED 1963, S. 337)

(e) Als Sozialistinnen und Sozialisten beabsichtigen wir, zu einem anerkannten Teil des gesellschaftlichen Widerstands gegen den Neoliberalismus zu werden und mit eigenen Vorstellungen und Projekten bei der Kräftigung von starken gesellschaftlichen Reformkräften mitzuwirken. Wir streben politische Bündnisse an, die diesen Zielen gerecht werden. (PDS 2003, S. 32) 
(f) Darüber hinaus streben wir eine Sicherung und Steigerung des Anteils der deutschen Seehäfen, der deutschen Seeschiffahrt und Luftfahrt am internationalen Verkehrsaufkommen an. (CDU 1971, S. 74)

(g) Die Sozialdemokratische Partei erstrebt eine Lebensordnung im Geiste dieser Grundwerte. (Godesberger Programm 1959, S. 69)

(h) Die Sozialistische Einheitspartei Deutschlands erstrebt die Entwicklung unseres Volkes zur gebildeten Nation. (SED 1963, S. 307)

(i) Die CSU erstrebt eine Politik der sozialen Bürgschaft, die die Hilfe der Gesellschaft mit der Eigenverantwortung des Bürgers verbindet. (CSU 1968, S. 235)

(j) Die sozialdemokratische Arbeiterpartei erstrebt die Errichtung des freien Volksstaates. (Eisenacher Programm 1869, S. 311)

(k) Von diesen Grundsätzen ausgehend, erstrebt die sozialistische Arbeiterpartei Deutschlands mit allen gesetzlichen Mitteln den freien Staat und die sozialistische Gesellschaft, [...]. (Gothaer Programm 1875, S. 313)

Lediglich zwei Textstellen, in denen in der Form direkter Sprechakte etwas versprochen wird, sowie zwei weitere, in denen etwas garantiert wird, können in den untersuchten Parteiprogrammen nachgewiesen werden (da die Äußerung (m) der SPD als Negation formuliert ist, bleibt als direktes VERSPRECHEN lediglich die Äußerung (l) der NSDAP). Auch die Verwendung des Ausdrucks verpflichtet bleiben in Äußerung (p) dient dazu, sich als Partei zukunftsorientiert festzulegen und dem Rezipienten die weitere Handlungsrichtung zu versichern.

(1) Die Führer der Partei versprechen, wenn nötig unter Einsatz des eigenen Lebens für die Durchführung der vorstehenden Punkte rücksichtslos einzutreten. (NSDAP 1920, S. 18)

(m) Wir versprechen nicht das Paradies auf Erden. (SPD 1989, S. 53)

(n) Die Nationaldemokratie garantiert volle unternehmerische Freiheit in sozialer Verpflichtung. (NPD 1971, S. 357)

(o) Wir garantieren das Grundrecht auf Kriegsdienstverweigerung. (SPD 1989, S. 16)

(p) Als demokratische Sozialistinnen und Sozialisten bleiben wir insbesondere jenen Traditionen der deutschen Arbeiterbewegung - der Gewerkschaften, der sozialdemokratischen wie der kommunistischen Parteien - verpflichtet, die in den Kämpfen gegen Nationalismus und Militarismus, gegen Imperialismus und Faschismus entstanden und sich 1945/1946 im gemeinsamen Einsatz für die antifaschistisch-demokratische Neuordnung Deutschlands bewährten. (PDS 2003, S. 31) 
Offensichtlich ist die verpflichtende Festlegung durch die Formulierung eines Versprechens oder einer Garantie ein sprachliches Handlungsmuster, das bei der Formulierung von Parteiprogrammen vermieden werden soll; die Häufigkeit der Verwendung dieser Sprachhandlungsmuster in den Parteiprogrammen legt diesen Schluss nahe. Erklärungen hierfür könnten sowohl in der Einschränkung des Handlungsspielraums liegen, als auch im berechtigten Rückbezug auf einen versprochenen oder garantierten Sachverhalt oder auf eine versprochene oder garantierte Handlung desjenigen, dem etwas versprochen oder garantiert wurde (denn dieser verlässt sich darauf, dass der Sprecher das Versprochene/Garantierte tun wird). Aufgrund dieser Feststellung müsste auch das oben angeführte Zitat von FLOHR (1968) abgeändert werden und die großen Versprechungen müssten durch den Ausdruck große Verheißungen oder noch schwächer Absichtserklärungen ersetzt werden. Wie im nachfolgenden Kapitel 2.1.5 zu sehen sein wird, richten sich die Parteiprogramme an einen möglichst großen Rezipientenkreis (an dieser Stelle ist FLOHR (1968) zuzustimmen), allerdings werden keine Versprechungen sondern vielmehr Verheißungen formuliert.

Der Ausdruck Versprechen wird von den Parteien allerdings dann verwendet, wenn eine Absichtserklärung des politischen Gegners offensichtlich nicht erfüllt wurde; mit diesem direkten Gegnerbezug geht bei einer solchen Formulierung ein Vorwurf oder eine Anklage einher und die Missstände des politischen Gegners werden betont. Indem in den Äußerungen (q) und ( $r$ ) statt von nicht realisierten Absichten oder geplanten Vorhaben vielmehr von nicht eingehaltenen Versprechen die Rede ist, dient die Verwendung dieses Ausdrucks dazu, den politischen Kontrahenten unglaubwürdig erscheinen zu lassen:

(q) Trotz aller in- und ausländischen Kritik, trotz vieler Zusicherungen und Versprechen den ,Radikalenerlaß' abschaffen zu wollen, sind seit 1972 Tausende von Mitbürgern aus politischen Gründen nicht eingestellt bzw. entlassen worden. (GRÜNE 1980, Kap. V, 1.5)

(r) Der neoliberale Kapitalismus versprach [...]. Der Neoliberalismus versprach [...]. Die neoliberalen Politiker haben ihre sozialen Versprechen nicht eingehalten. (PDS 2003, S. 7)

\subsubsection{Forderungen}

Sowohl das Godesberger Programm als auch die anderen Parteiprogramme bestehen überwiegend aus Formulierungen, die als Verheißungen oder als Forderungen konzipiert sind; die Häufigkeit der jeweiligen Verwendung wird durch die Zahl in Klammern widergespiegelt (diese bezieht sich jedoch nur auf die Verwendung im Godesberger Programm und soll durch die Häufigkeiten verdeutlichen, dass die expliziten Forderungen durch andere Satzmuster ersetzt werden). Beispiele aus den anderen 
Parteiprogrammen werden gegebenenfalls angefügt. Viele der formulierten Äußerungen werden im Godesberger Programm als Grundforderungen (5) (die in einer menschenwürdigen Gesellschaft erfüllt sein müssen) bezeichnet.

\section{A Gebrauch des Verbs fordern (3) und verlangen}

Erwartungsgemäß werden Forderungen in den Parteiprogrammen durch den Gebrauch der Verben fordern und verlangen explizit formuliert. Die Verwendung des Wortes verlangen als direkter Sprechakt ist jedoch selten, obwohl das Verb verlangen häufig verwendet wird, um (Zustands)beschreibungen und Darlegungen zu äußern oder allgemeine Ansichten oder Aussagen zu formulieren, wie beispielsweise: „Demokratische Kontrolle der wirtschaftlichen Macht des Kapitals verlangt einen handlungsfähigen Staat, starke Gewerkschaften und Mitbestimmung" (SPD 1989, S. 37).

Die Äußerungen (d) und (e) sind die einzigen, die als direkte Forderung der Parteien mit Verwendung von verlangen nachgewiesen werden können:

(9) (a) Die Sozialdemokratische Partei fordert die völkerrechtliche Ächtung der Massenvernichtungsmittel auf der ganzen Welt. [...] Die Sozialdemokratische Partei Deutschlands fordert eine allgemeine und kontrollierte Abrüstung und eine mit Machtmitteln ausgestattete internationale Rechtsordnung, die nationale Landesverteidigungen ablösen wird. (Godesberger Programm 1959, S. 73)

(b) Wir fordern eine wirkungsvollere Organisation und verstärkte Zusammenarbeit von Bund, Ländern und Gemeinden, Wirtschaft und Wissenschaft zur Lösung dieser Aufgaben. (CDU 1971, S. 86)

(c) Wir halten unsere Forderung nach einem Recht der Frauen auf selbstbestimmte Schwangerschaft uneingeschränkt aufrecht [...]. (PDS 2003, S. 15)

(d) Die PDS verlangt, in allen Medien, die Einfluss auf die demokratische Meinungs- und Willensbildung haben, öffentlichrechtliche Angebote zu schaffen oder zu erhalten. (PDS 2002, S. 29)

(e) Wir verlangen von allen Medien eine Sprache und Begrifflichkeit, die der tatsächlichen Situation der Überlebenden und der Angehörigen der Toten gerecht wird und die Gewalt, der sie ausgesetzt waren, beim Namen nennt. (DIE FRAUEN 2002, S. 30)

Der Gebrauch des Verbs fordern ist häufig und kann parteiübergreifend nachgewiesen werden, jedoch kann auch hier lediglich ein knapper Überblick geboten werden. Hingewiesen soll auf den Ausdruck eine For- 
derung aufrecht halten in Äußerung (c) als Sonderform einer (erneuten) Forderung.

Im Gegensatz zur expliziten Verwendung der Verben fordern und verlangen kann eine Forderung jedoch auch implizit erfolgen:

(f) Erst in einem wiedervereinigten Deutschland wird das ganze Volk in freier Selbstbestimmung Inhalt und Form von Staat und Gesellschaft gestalten können. (Godesberger Programm 1959, S. 71)

Bei der oben zitierten Aussage (f) handelt es sich um eine Behauptung die besagt, dass eine Gestaltung von Staat und Gesellschaft in freier Selbstbestimmung nur dann möglich ist, wenn die Teilung der Bundesrepublik Deutschland überwunden wird; das Wort erst signalisiert dabei, dass der angestrebte Zustand zum Zeitpunkt der Formulierung noch nicht erreicht ist. Aus diesen Bedingungsfaktoren ergibt sich somit die implizite Forderung nach der Wiedervereinigung Deutschlands; unter formalem Gesichtspunkt müsste die Äußerung (d) jedoch als Verheißung oder Versprechen gewertet werden.

Der überwiegende Teil der Forderungen des Parteiprogramms erfolgt allerdings weder auf explizitem noch auf implizitem Weg; die meisten Forderungen folgen einem abweichenden Konstruktionsschema unter Verwendung eines mehrteiligen Prädikats (sowohl Aktiv als auch Passiv). Dabei verzichten die Formulierungen auf die Verwendung des Verbs fordern. Aufgrund der Vielzahl dieser Formulierungen können lediglich einige exemplarische Beispielsätze des Parteiprogramms zitiert werden um die bestimmten Konstruktionsmuster zu veranschaulichen.

\section{B 1 Satzmuster Modalverb müssen + Infinitiv Aktiv (10); Vollverb Passiv (15)}

(10) (a) Alle Völker müssen sich einer internationalen Rechtsordnung unterwerfen, die über eine ausreichende Exekutive verfügt. [...] (Aktiv)

(b) Alle Völker müssen die gleiche Chance haben, am Wohlstand der Welt teilzunehmen. [...] (Aktiv)

(c) Die demokratischen Staaten müssen ihre Solidarität vor allem mit den Entwicklungsländern bekunden. (Aktiv) (Godesberger Programm 1959, S. 85)

(d) Alle Vorrechte im Zugang zu Bildungseinrichtungen müssen beseitigt werden. (Passiv) (Godesberger Programm 1959, S. 70)

(e) In der Demokratie müssen die Rechte der Minderheit neben den Rechten der Mehrheit gewahrt werden. (Passiv) (Godesberger Programm 1959, S. 71) 
Einige Formulierungen des oben dargestellten Satzmusters B 1 sind gleichbedeutend mit den weiter unten dargestellten Satzmustern F 1 und F 2. Ohne Bedeutungsunterschied lassen sich die Äußerungen (d) und (e) derart umformulieren, dass sie mit den Mustern F 1 und F 2 übereinstimmen:

1. Alle Vorrechte im Zugang $\mathrm{zu}$ Bildungseinrichtungen sind $\underline{\text { zu}}$ beseitigen. (vgl. 10 (d))

2. In der Demokratie sind die Rechte der Minderheit neben den Rechten der Mehrheit zu wahren. (vgl. 10 (e))

\section{B 2 Satzmuster Modalverb muss + Infinitiv Aktiv (20); Vollverb Passiv(10)}

(11) (a) Im demokratischen Staat muß sich jede Macht öffentlicher Kontrolle fügen. (Aktiv) Das Interesse der Gesamtheit muß über dem Einzelinteresse stehen. [...] (Aktiv) (Godesberger Programm 1959, S. 70)

(b) Die Wirtschaftspolitik muß auf der Grundlage einer stabilen Währung die Vollbeschäftigung sichern, die volkswirtschaftliche Produktivität steigern und den allgemeinen Wohlstand erhöhen. (Aktiv)

(c) Um alle Menschen am steigenden Wohlstand zu beteiligen, $\underline{\text { muß }}$ die Wirtschaft den ständigen Strukturveränderungen planmäßig angepaßt werden, damit eine ausgeglichene Wirtschaftsentwicklung erreicht wird. (Passiv) (Godesberger Programm 1959, S. 74)

(d) Der Rückstand in der sozialen Gesetzgebung muß beseitigt werden. (Passiv) (Godesberger Programm 1959, S. 78)

\section{1 Satzmuster Modalverb sollen + Infinitiv Aktiv (11); Vollverb Passiv (2)}

(12) (a) Nur Begabung und Leistung sollen jedem den Aufstieg ermöglichen. [...] (Ak-tiv) (Godesberger Programm 1959, S. 70)

(b) Die Grundrechte sollen nicht nur die Freiheit des einzelnen gegenüber dem Staat sichern, sie sollen als gemeinschaftsbildende Rechte den Staat mitbegründen. (Aktiv) (Godesberger Programm 1959, S. 71)

(c) Die Mitwirkung der Eltern in der Schulerziehung und eine Mitverwaltung der Schüler sollen an allen Schulen ausgebaut werden. [...] (Passiv)

(d) Alle Lehrer sollen an wissenschaftlichen Hochschulen ausgebildet werden. (Passiv) (Godesberger Programm 1959, S. 82) 


\section{2 Satzmuster Modalverb soll + Infinitiv Aktiv (9); Vollverb Passiv (3)}

(13) (a) Der Staat soll Vorbedingungen dafür schaffen daß der einzelne sich in freier Selbstverantwortung und gesellschaftlicher Verpflichtung entfalten kann. (Aktiv) (Godesberger Programm 1959, S. 71)

(b) Die Kulturpolitik des Staates soll alle kulturwilligen Kräfte ermutigen und fördern. (Aktiv) (Godesberger Programm 1959, S. 81)

(c) Durch solche Unternehmen soll den Interessen der Allgemeinheit Geltung verschafft werden. (Passiv) (Godesberger Programm 1959, S. 75)

(d) Deshalb soll das Gemeineigentum nach den Grundsätzen der Selbstverwaltung und der Dezentralisierung geordnet werden. (Passiv) (Godesberger Programm 1959, S. 76)

Die Betrachtung der oben dargestellten Satzmuster C 1 und C 2 unter dem Aspekt der Forderung ist nicht unproblematisch, da sich die Äußerungen möglicherweise auch als Versprechen deuten lassen. So kann beispielsweise die Äußerung (a) unter Punkt C 1 sowohl als Forderung als auch als Versprechen der SPD gegenüber der Bevölkerung verstanden werden. Die Forderung beinhaltet, dass hinsichtlich des beruflichen oder gesellschaftlichen Aufstiegs lediglich Leistung und Begabung zu bewerten und andere Faktoren (Herkunft, persönliche Beziehungen, finanzielles Vermögen u.a.) zu vernachlässigen sind. Die Äußerung kann als Versprechen der SPD verstanden werden, die damit versichert, politische Maßnahmen zu ergreifen um den Aufstieg durch Leistung und Begabung als einzige Voraussetzungen überhaupt erst zu ermöglichen. Weiterhin kann die Äußerung (c) unter Punkt C 1 nicht direkt als Forderung, sondern vielmehr als Absichterklärung sowie als Zusage der SPD verstanden werden; die SPD sagt dieser Interpretation folgend der Bevölkerung zu (sie verspricht), dass die Mitwirkung der Eltern in der Schulerziehung und eine Mitverwaltung der Schüler als konkretes Planungsvorhaben bereits existiert bei einem bevorstehenden Wahlsieg der SPD in die Realität umgesetzt wird. Auch die Äußerung (d) unter Punkt C 1 bleibt bei eingehender Betrachtung bedingt unklar: Wenn Lehrer bis zum Zeitpunkt der Konzeption des Godesberger Programms nicht an wissenschaftlichen Hochschulen ausgebildet wurden, dann drückt Äußerung (d) mit der Verwendung des Satzmusters sollen + Vollverb + Passiv eine Forderung aus. Wenn Lehrer jedoch zum Zeitpunkt der Formulierung bereits die universitäre Laufbahn beschreiten, dann kann Äußerung (d) als Versprechen oder Zusage verstanden werden, nämlich dass für Lehrer diese Form der Berufsausbildung auch in Zukunft gewährleistet ist und dass alle Lehrer einen Hochschulabschluss haben sollen. Die letzte Interpretationsvariante ist denkbar, jedoch legt das Fehlen eines Wortes wie weiterhin die Interpretation als Forderung nahe. 


\section{1 Satzmuster Modalverb dürfen + Negation + Infinitiv Aktiv (7); Vollverb Passiv (1)}

(14) (a) Die Parlamente, die Verwaltung und die Rechtsprechung dürfen nicht unter den einseitigen Einfluß von Interessenvertretungen fallen. (Aktiv) (Godesberger Programm 1959, S. 72)

(b) Die Streitkräfte dürfen nur der Landesverteidigung dienen. (Aktiv) (Godesberger Programm 1959, S. 73)

(c) Regional begrenzte übernationale Gemeinschaften dürfen nicht zur Abschließung der Außenwelt führen. (Aktiv) (Godesberger Programm 1959, S. 84)

(d) Gesellschaftliche Zustände, die zu individuellen und sozialen Notständen führen, dürfen nicht als unvermeidlich und unabänderlich hingenommen werden. (Passiv) (Godesberger Programm 1959, S. 78/79)

D 2 Satzmuster Modalverb darf + Negation + Infinitiv Aktiv (4); Vollverb Passiv (2)

(15) (a) Der Krieg darf kein Mittel der Politik sein. (Aktiv) (Godesberger Programm 1959, S. 70)

(b) Die Bundesrepublik Deutschland darf atomare und andere Massenvernichtungsmittel weder herstellen noch verwenden. (Aktiv) (Godesberger Programm 1959, S. 73)

(c) Eine religiöse oder weltanschauliche Verkündigung darf nicht parteipolitisch oder $\mathrm{zu}$ antidemokratischen Zwecken mißbraucht werden. (Passiv) (Godesberger Programm 1959, S. 81)

(d) Die künstlerische Entfaltung darf durch kein Reglement, insbesondere durch keine Zensur, beschränkt werden. (Passiv) (Godesberger Programm 1959, S. 83)

\section{E 1 Satzmuster Hilfsverb haben $+z u+$ Infinitiv (2)}

(16) (a) Staat und Gesellschaft haben die Familie zu schützen, $\underline{\mathrm{zu}}$ fördern und zu stär-ken. (Godesberger Programm 1959, S. 80)

(b) Die Berufsschulen haben nicht nur der fachlichen, sondern auch der allgemeinen und staatsbürgerlichen Bildung und Erziehung zu dienen. (Godesberger Programm 1959, S. 82) 


\section{E 2 Satzmuster Hilfsverb hat $+z u+$ Infinitiv (4)}

(17) (a) Jeder Bürger hat die Überzeugung seiner Mitmenschen $\underline{\mathrm{zu}}$ achten. $[\ldots]$

(b) Als Sozialstaat hat er für seine Bürger Daseinsvorsorge zu treffen, um jedem die eigenverantwortliche Selbstbestimmung zu ermöglichen und die Entwicklung einer freiheitlichen Gesellschaft $\mathrm{zu}$ fördern. (Godesberger Programm 1959, S. 71)

(c) Sozialpolitik hat wesentliche Voraussetzungen dafür $\underline{\mathrm{zu}}$ schaffen, daß sich der einzelne in der Gesellschaft frei entfalten und sein Leben in eigener Verantwortung gestalten kann. (Godesberger Programm 1959, S. 78)

(d) Der Staat hat Vorsorge zu treffen, daß Forschungsergebnisse nicht zum Schaden der Menschheit mißbraucht werden. (Godesberger Programm 1959, S. 81)

Zwar wird im nachfolgenden Kapitel ausführlich auf die Verwendung von Appellen eingegangen; es sei an dieser Stelle dennoch angemerkt, dass die Äußerung (a) unter Punkt E 2 aufgrund des dargestellten Satzmusters durchaus als Forderung verstanden werden kann. Aufgrund der direkten Erwähnung durch das Indefinitpronomen jeder und das Substantiv Bürger und dessen Achtung gegenüber den Mitmenschen kann die Äußerung jedoch auch als Appell an das moralische oder ethische Empfinden des Wählers verstanden werden.

\section{F 1 Satzmuster Hilfsverb ist + zu + Infinitiv (15)}

(18) (a) Die kulturelle, wirtschaftliche und soziale Lage der gesamten Landbevölkerung ist zu verbessern. (Godesberger Programm 1959, S. 78)

(b) So ist die im Arbeitsleben erreichte Lebenshaltung zu $\underline{\text { si- }}$ chern. (Godesberger Programm 1959, S. 79)

(c) Künstlerischem Schaffen ist volle Freiheit zu gewähren. (Godesberger Programm 1959, S. 83)

\section{$\underline{\text { F } 2}$ Satzmuster Hilfsverb sind + zu + Infinitiv (12)}

(19) (a) An der Rechtspflege sind ehrenamtliche Richter gleichberechtigt $\underline{\mathrm{zu}}$ beteiligen. (Godesberger Programm 1959, S. 72)

(b) Alle sozialen Geldleistungen, auch die Renten der Kriegsbeschädigten und Kriegshinterbliebenen, sind der Entwicklung der steigenden Arbeitseinkommen laufend anzupassen. [...]

(c) Lebensbedingungen und Lebensformen sind so zu gestalten und die Gesundheitspolitik ist so aufzubauen, daß ein Leben in Gesundheit möglich wird. (Godesberger Programm 1959, S. 79) 
Abschließend soll an dieser Stelle noch ein weiteres Satzmuster erwähnt werden, dass sich hinsichtlich der Bestimmtheit der Formulierung von den bisher dargestellten Mustern unterscheidet, wenn auch nur geringfügig. Die Verwendung des Modalverbs können/kann wirkt weniger direkt und verpflichtend, die nachfolgend zitierten Forderungen (a) und (b) haben einen geringeren Grad an Bestimmtheit als die übrigen Forderungen. Insbesondere die Verwendung des Modalverbs sollten wirkt zurückhaltender und erweckt im Vergleich zu den fordernden Ausdrücken soll und sollen sowie muss und müssen vielmehr den Eindruck eines Vorschlags; das Modalverb sollten im 2. Konjunktiv drückt aus, "dass etw[as] Bestimmtes wünschenswert, richtig, vorteilhaft o.ä. wäre." ${ }^{182}$ Die Verwendung von sollten im konjunktivischen Modus ist daher ein Mittel der Zurückhaltung und ermöglicht der SPD, sich von der Verantwortung für die Richtigkeit des Inhalts zu distanzieren und anstelle einer Forderung einen Wunsch zu artikulieren. Auch die Formulierung in Äußerung (c) erweckt durch die Verwendung von sollte vielmehr den Eindruck eines Vorschlags als den einer Forderung.

\section{G Satzmuster Modalverb können (1)/kann (1) + Negation Infinitiv;} sollte (1)

(20) (a) Dieser Verantwortung für den Wirtschaftsablauf kann sich der Staat nicht entziehen. (Godesberger Programm 1959, S. 74)

(b) Die Hochschulen können aber nicht isoliert von der übrigen Lebenswirklichkeit bestehen und sollten darum mit anderen Institutionen der demokratischen Gesellschaft, insbesondere mit den Einrichtungen der Erwachsenenbildung, zusammenarbeiten. (Godesberger Programm 1959, S. 83)

(c) Wo betriebliche Ertragsbeteiligung möglich ist, sollte sie gefördert werden. (CDU 1971, S. 71)

Aufgrund der Frequenz von Zielbestrebungen und Forderungen in allen untersuchten Parteiprogrammen kann an dieser Stelle die These formuliert werden, dass diese beiden sprachlichen Handlungsmuster zur Texttradition von Parteiprogrammen zu gehören scheinen. Unabhängig von der zeitlichen Komponente finden sich in allen Parteiprogrammen (von 1869 bis 2003) eine Vielzahl von Forderungen und Zielbestrebungen.

Da die oben zitierten Forderungen häufig appellativen Charakter haben und der Hinweis auf den Appell als besondere Ausprägung einer Forderung bereits geliefert wurde, soll im folgenden Kapitel der Versuch einer Begriffsdifferenzierung unternommen werden. 


\subsubsection{Aufforderungen/Appelle}

Die Betrachtung des appellativen Stils im Godesberger Programm soll durch das folgende, anschauliche Zitat eingeleitet werden:

Formal gesehen, besteht der appellierende Stil aus Prämissen und Schlußfolgerungen, die zum Teil explizit geäußert, zum Teil implizit unterstellt werden. Die Schlußfolgerung besteht in der Regel in einer positiven oder negativen Aussicht auf die Zukunft und legt dem Bürger einen entsprechenden politischen Einsatz nahe. ${ }^{183}$

Aus der Intention des Parteiprogramms, möglichst viele Wähler mit den formulierten Zielen und Inhalten anzusprechen und davon zu überzeugen, dass die von der eigenen Partei praktizierte Politik allgemein akzeptiert wird, resultieren als sprachliche Handlungsmuster neben den Argumenten und Behauptungen auch Aufforderungen und Appelle; ein Appell ist daher als die "pragmatischste aller Sprachfunktionen“184 $\mathrm{zu}$ bewerten.

Grundsätzlich ist festzuhalten, dass der Typus der appellierenden (oder appellativen) Sprache mit der Überzeugungsfunktion bzw. der Beeinflussungsfunktion im Sinne der Willensbildung einer Argumentation einhergeht. Durch die Verwendung von Appellen werden bestimmte Adressatengruppen zu politischer Unterstützung aufgerufen (beispielsweise im Zuge von Wahlkämpfen, in Parlamentsdebatten, aber auch bei politischen Diskussionen allgemein wie etwa im Freundes- oder Familienkreis); ein Appell lässt sich daher als eine bestimmte und direkt an den Rezipienten gerichtete Form der Forderung betrachten. Die Appellfunktion lässt sich folgendermaßen verdeutlichen:

Der Emittent gibt dem Rezipienten zu verstehen, daß er ihn dazu bewegen will, eine bestimmte Einstellung einer Sache gegenüber einzunehmen (Meinungsbeeinflussung) und/oder eine bestimmte Handlung zu vollziehen (Verhaltensbeeinflussung). ${ }^{185}$

Hinsichtlich des Rezipienten fällt auf, dass die appellativen Muster im Godesberger Programm überwiegend einen allgemeinen, sich an alle potentiellen Wähler richtenden Charakter haben. Zwar kann die appellative Textfunktion durch die Paraphrase „Ich (der Emittent) fordere dich (den Rezipienten) auf, die Einstellung (Meinung) X zu übernehmen/die Handlung X zu vollziehen"186 sowie durch die Verwendung performati-

183 Edelman 1976, S. 170

184 Polenz, Peter von: Deutsche Satzsemantik. Grundbegriffe des Zwischen-denZeilen-Lesens. Berlin/New York 1985, S. 67

185 Brinker, Klaus: Linguistische Textanalyse. Eine Einführung in Grundbegriffe und Methoden. Berlin 52001, S. 112

186 Brinker 2001, S, 112 
ver Formeln mit den Verben ANORDNEN, BEFEHLEN, RATEN, EMPFEHLEN, FRAGEN, BEANTRAGEN, oder BEAUFTRAGEN explizit gemacht werden, der Gebrauch solcher expliziter Strukturen ist jedoch allgemein selten. In den untersuchten Parteiprogrammen sind folgende Verben nachweisbar: BITTEN, WARNEN, BILLIGEN, FESTSTELLEN, GESTATTEN, DANKEN, VORWERFEN, UNTERSTÜTZEN, AUFFORDERN und VERLANGEN.

Die Differenzierung der genannten Termini fällt dabei nicht leicht. Insbesondere ergibt sich die Schwierigkeit, Appelle und Aufforderungen von Forderungen abzugrenzen. Diese Problematik wird insbesondere bei Betrachtung des Appells in der parenthetischen Äußerung (d) deutlich, die dem im vorangegangenen Kapitel dargestellten Satzmuster D 2 der Forderungen entspricht. In den Parteiprogrammen lassen sich folgende Appelle nachweisen (einige Äußerungen mit appellativem Charakter werden im nachfolgenden Kapitel 2.1.5.1.1 aufgegriffen):

(21) (a) Diesen Widerspruch aufzulösen, sind wir Menschen aufgerufen. In unsere Hand ist die Verantwortung gelegt für eine glückliche Zukunft oder für die Selbstzerstörung der Menschheit. (Godesberger Programm 1959, S. 69)

(b) Jeder, Mann und Frau, ist aufgerufen, hier und in allen Ländern der Erde. (Godesberger Programm 1959, S. 87)

(c) Wir dürfen die Stabilität der Ökosysteme nicht zerstören. [...] Wir müssen die Vergewaltigung der Natur aufgeben, um mit ihr zu überleben. (GRÜNE 1980, Kap. I/Kap. IV, 1)

(d) Menschliches Leben $\underline{\text { darf }}$ - wie Leben überhaupt - nicht patentiert werden! (DIE FRAUEN 2002, S. 37)

Offensichtlich hat die Verwendung des Verbs aufrufen insbesondere in der 1. Person Plural in (a) (wir sind aufgerufen) eine integrierende Wirkung und kann als Indikator eines Appells betrachtet werden. Der Appell vollzieht sich hinsichtlich der sprachlichen Handlung lediglich innerhalb des unterstrichenen Teils, allerdings erschließt sich der appellative Sachverhalt bei isolierter - d.h. dem Kontext entnommener Betrachtung nicht vollständig. Streng genommen ist daher die weitere Ausführung in (a) ebenso als Bestandteil des Appells zu betrachten, da durch die Erwähnung der eigenverantwortlichen Selbstzerstörung des Menschen sowie der Alternative der glücklichen Zukunft an das moralisch vertretbare und richtige Handeln des Menschen appelliert wird; der zweite Teil der Äußerung (a) begründet den vorangegangenen Appell und rechtfertigt diesen dadurch. Die Äußerung (a) ist als ein Aufruf zu verstehen, der neben der Mitteilung seiner Stellungnahmen (Informationsfunktion) zugleich intendiert, dass der Rezipient seine Bewertung des Sachverhalts übernimmt und danach handelt; die Äußerung (a) ist in 
der Form eines Aufrufs ein Appell an eine bestimmte Meinungsübernahme (die der SPD) sowie an die einhergehenden Handlungsmöglichkeiten des Bürgers in der Gesellschaft (hier: aller Menschen).

Die integrierende Wirkung wird in (b) durch die Verwendung des substantivischen Indefinitpronomens jeder hergestellt; das Subjekt dieses Satzes bezieht sich auf die gesamte Bevölkerung, d.h. auch auf alle Wähler, was weiterhin durch die Apposition Mann und Frau nochmals betont wird (vgl. Kapitel 2.2.2.4 Appositionen). Dadurch wird einem möglichen Vorwurf der Geschlechterdifferenzierung prophylaktisch entgegengewirkt.

Auch bei Betrachtung der Äußerungen (c) und (d) fällt auf, dass Appell und Forderung lediglich schwer voneinander abzugrenzen sind. So beinhaltet der offensichtliche Appell in (c) die implizite (Auf-)Forderung, die Umwelt zu schonen oder zu schützen und appelliert an das Umweltbewusstsein des Bürgers oder besser des Verbrauchers; die auffordernde Wirkung ist durch den Gebrauch des Ausdrucks wir müssen jedoch deutlicher als bei wir dürfen nicht.

\subsubsection{Anklagen/Vorwürfe}

Charakteristisch für die politische Sprachverwendung ist die Referenz auf den politischen Gegner. Mit der Eigenwerbung einer Partei ist die DELEGITIMATION des politischen Gegners verbunden. Konkret stellt sich eine solche Konkurrenzstrategie dar, wenn eine Partei die Misserfolge des politischen Gegners erwähnt oder der gegnerischen Partei Dilettantismus vorwirft, wie in nachfolgend zitierter Textstelle (a) zu sehen ist. Bei Betrachtung der Textstelle (b) wird deutlich, dass die Anklage des politischen Gegners mit der Formulierung einer Behauptung vollzogen wird.

(22) (a) Die in Bonn etablierten Parteien verhalten sich, als sei auf dem endlichen Plane-ten Erde eine unendliche industrielle Produktionssteigerung möglich. Dadurch führen sie uns nach eigener Aussage vor die ausweglose Entscheidung zwischen Atomstaat oder Atomkrieg, zwischen Harrisburg oder Hiroshima. [...] Gegenüber der eindimensionalen Produktionssteigerungspolitik vertreten wir ein Gesamtkonzept. (GRÜNE 1980, Kap. I)

(b) Die Kapitalisten aller Länder, das sind die wahren Anstifter zum Völkermord. (KPD 1918/19, S. 107) 


\subsection{Expliziter Gegnerbezug}

In der öffentlichen politischen Kommunikation geht es neben der Werbung und Selbstdarstellung vor allem darum, Zustimmung beim Wähler dadurch zu erzielen, dass Argumente für die eigene und gegen die Position des politischen Gegners vorgebracht werden. Auch die Konzeption eines Parteiprogramms orientiert sich an der Programmatik einer anderen Partei oder an den Aussagen der politischen Gegner allgemein. Insbesondere Wahlprogramme thematisieren die Aussagen und Argumente der politischen Hauptkonkurrenten. Als direkte Bezugnahme versucht insbesondere der Typus des Wahlprogramms mit dieser Methode, die eigene Politik gegenüber der Politik anderer Parteien abzugrenzen sowie potentielle Wähler zu werben und zu überzeugen. Die Notwendigkeit, die gegnerische Seite als ablehnenswert oder gar verachtenswert darzustellen, ergibt sich aus diesen Handlungszielen; solche Darstellungen sind meist polemisch angehaucht.

Die Abgrenzung gegenüber der gegnerischen Seite kann durch expliziten Bezug auf sie geschehen. Ein solcher expliziter Gegnerbezug lässt sich auch in einem Grundsatzprogramm wie dem Godesberger Programm nachweisen. Darin ist von der brutalen kommunistischen Herausforderung (vgl. Äußerung (b)) die Rede:

(23) (a) Zu Unrecht berufen sich die Kommunisten auf sozialistische Traditionen. In Wirklichkeit haben sie das sozialistische Gedankengut verfälscht. Die Sozialisten wollen Freiheit und Gerechtigkeit verwirklichen, während die Kommunisten die Zerrissenheit der Gesellschaft ausnutzen, um die Diktatur ihrer Partei zu errichten. (Godesberger Programm 1959, S. 70)

(b) Die alten Kräfte erweisen sich als unfähig, der brutalen kommunistischen Herausforderung das überlegene Programm einer neuen Ordnung politischer und persönlicher Freiheit und Selbstbestimmung, wirtschaftlicher Sicherheit und sozialer Gerechtigkeit entgegenzustellen. [...]

(c) Sie [die alten Kräfte] wehren sich gegen die Lockung der Kommunisten, die sie in ihren Machtbereich einzubeziehen versuchen.

(d) Die Kommunisten unterdrücken die Freiheit radikal. Sie vergewaltigen die Menschenrechte und das Selbstbestimmungsrecht der Persönlichkeit und der Völker. Gegen ihren Machtapparat stellen sich heute zunehmend auch die Menschen der kommunistisch regierten Länder selber. (Godesberger Programm 1959, S. 86)

Die SPD übernimmt in ihrer Programmatik des Godesberger Programms zentrale Punkte der CDU-Regierung, wie beispielsweise die Bejahung der Marktwirtschaft, der Bundeswehr und der Kirchen; auf diese Weise 
weicht die SPD als Volkspartei von ihrer ursprünglichen Ausrichtung ab. Die Verneinung des Kommunismus als Ideologie bzw. Regierungsform resultiert zwangsläufig aus dieser neuen politischen Haltung. Jedoch gehen die Aussagen in der oben zitierten Textpassage über die bloße Distanzierung hinaus. Die negative und polemische Darstellung des politischen Gegners resultiert daraus, „daß in der parlamentarischen Demokratie das urdemokratische Prinzip des Meinungsstreits in Form von Parteienkonkurrenz institutionalisiert ist. "187

Als Gegner können aber auch die im Parteiprogramm als Bedrohung dargestellten wirtschaftlichen Großunternehmen (2) bzw. die Großwirtschaft (4) angesehen werden; ausgewählte Textbeispiele sind nachfolgend wiedergegeben:

(24) (a) Wo das Großunternehmen vorherrscht, gibt es keinen freien Wettbewerb. [...]

(b) Mit ihrer durch Kartelle und Verbände noch gesteigerten Macht gewinnen die führenden Männer der Großwirtschaft einen Einfluß auf Staat und Politik, der mit demokratischen Grundsätzen nicht vereinbar ist. Sie usurpieren Staatsgewalt. Wirtschaftliche Macht wird zu politischer Macht. [...]

(c)Die Bändigung der Macht der Großwirtschaft ist darum zentrale Aufgabe einer freiheitlichen Wirtschaftspolitik. Staat und Gesellschaft dürfen nicht zur Beute mächtiger Interessengruppen werden. [...]

(d) Leistungsfähige mittlere und kleine Unternehmen sind $\mathrm{zu}$ stärken, damit sie die wirtschaftliche Auseinandersetzung mit den Großunternehmen bestehen können. (Godesberger Programm 1959, S. 75)

Mit der ablehnenden Haltung der SPD gegenüber dieser bestimmten Form der wirtschaftlichen Realität, von der sich die Partei in (a) und (b) distanziert, geht in den Äußerungen (c) und (d) als Bestrebungen und Zielvorgaben einher, die Bevölkerung vor dieser Entwicklung zu bewahren. Offensichtlich wird mit der Erwähnung dieser Ausdrücke ein spezielles Thema aufgegriffen, das die SPD gemäß ihrer Parteiphilosophie für zugkräftig hält, das aber auch parteiübergreifend charakteristisch für die politische Sprachverwendung im Wahlkampf ist:

In der Tat gibt es überzeugende Beweise für die Existenz eines politischen Interesses an der ständigen Wiederholung der Versicherung, daß man gegen als mächtig und bedrohlich empfundene wirtschaftliche Kräfte geschützt wird. ${ }^{188}$

\footnotetext{
187 Klein 1996b, S. 11

188 Edelman 1976, S. 33
} 


\subsubsection{Sonderformen politischer Sprachverwendung}

Wie bereits erläutert stellt die politische Sprachverwendung eine Sonderform aller Kommunikationsformen dar. Zwar wird der Handlungsspielraum der politischen Kommunikation auch durch Kommunikationsnormen (Konversationsmaximen) geprägt, es wirken allerdings Faktoren wie Medienbedingungen oder die Parteiräson mit oftmals stärkerer Ausprägung auf die politische Kommunikation ein. Ein weiterer außersprachlicher Faktor ist die Rollenvielfalt der politischen Akteure, da diese oftmals neben der Partei mit Unternehmen, Interessengruppen oder anderen Institutionen verklammert sind: So ist ein Abgeordneter

Mitglied einer Partei, die Regierungs- oder Oppositionspartei ist, was auf Bundes- und Länderebene verschieden sein kann; dabei gehört er zu bestimmten Gemeinde-, Kreis-, Landesgliederungen und informellen ,Flügeln' mit jeweiligem politischen und kulturellen Kolorit; er ist Mitglied von einer oder mehreren Interessengruppen, häufig auch noch Angehöriger einer Berufsgruppe, und in jedem Fall durch Herkommen und Sozialisation subkulturell verankert. ${ }^{189}$

Da Politiker häufig auch Mitglied in Unternehmensvorständen sind oder in Aufsichtsräten sitzen, ergibt sich aus einer solchen Konstellation ein Spannungsgefüge, das eine Orientierung in viele Richtungen erforderlich macht: Politisches Handeln ist daher in der Regel „,mehrfachadressiert', ,mehrfachzielgerichtet' und damit handlungsmäßig ambivalent. “190 Daher muss ein Politiker

die Kunst perfektionieren lernen, Mehrfachadressierungen und Mehrfachfunktionen von Äußerungen auch als Kompromißbildungen bei solchen multilateralen Anforderungen zu nutzen. Dies führt natürlich andererseits zur vielbeklagten Formelhaftigkeit und Vagheit politischer Aussagen, die eben oft nur noch den kleinsten gemeinsamen Nenner der verschiedenen Rollenerwartungen enthalten, aus der jeweils absolut gesetzten Einzelperspektive aber häufig als Verrat wirken oder einfach als unglaubwürdig. ${ }^{191}$

Die beschriebenen Voraussetzungen haben bestimmte Auswirkungen auf die politische Sprachverwendung. Nach einem Exkurs in die Theorie der Kommunikationsnormen sollen in den folgenden Kapiteln die unterschiedlichen Auswirkungen der außersprachlichen Faktoren auf die Konzeption des Parteiprogramms dargestellt werden.

Der Sprachphilosoph GRICE (1979) unternimmt einen Versuch, die sprachlichen Handlungsnormen als kommunikative Ethik zu postulieren

189 Holly 1990, S. 54

190 Burkhardt, Armin: Das Parlament und seine Sprache. Studien zur Theorie und Geschichte parlamentarischer Kommunikation. Tübingen 2003, S. 119

191 Holly 1990, S. 54 
und greift dabei die Tradition der Logik und der Sprachphilosophie auf. GRICE (1979) formuliert eine Art Rahmenkonzept um zu verdeutlichen, wie die einzelnen Postulate im Kontext einer kommunikativen Situation eingesetzt werden. Dabei ist davon auszugehen, dass Kommunikation als kooperatives Handeln verstanden wird und dass alle an der Kommunikation Beteiligten ein minimales gemeinsames Interesse daran haben, verstanden $\mathrm{zu}$ werden (cooperative principle). Die Regel dieses Prinzips lautet: „Mache deinen Gesprächsbeitrag jeweils so, wie es von dem akzeptierten Zweck oder der akzeptierten Richtung des Gesprächs, an dem du teilnimmst, gerade verlangt wird." 192

Die vorausgesetzte Kooperationsbereitschaft beim Rezipienten führt dementsprechend als "Reparaturverfahren“193 zu einer Um- bzw. Reinterpretation (Schlussprozess), wenn der Wortlaut einer Äußerung dem Kooperationsprinzip nicht zu entsprechen scheint, so dass die Übereinstimmung mit dem Kooperationsprinzip wiederhergestellt ist. Das Ergebnis dieses Umdeutungsverfahrens ist die konversationelle Implikatur (conversational implicatures): ein vom Sprecher in einer bestimmten kommunikativen Verwendung an eine bestimmte Äußerung geknüpfter, aber nicht ausgedrückter Sinn, den es zu erschließen gilt. Folglich ist ein solcher Sinn von der Verwendungssituation abhängig und nicht konventionell an Äußerungen gebunden.

Das abstrakte Prinzip, „,wie Kommunizierende ihre Kenntnisse der Sprache, der Sprechaktregeln und eventueller weiterer konkreter Sprachgebrauchsregeln zum Tragen bringen “194, entfaltet GRICE (1979) in den so genannten Konversationsmaximen, die die Kriterien, denen Kommunikationsbeiträge zu genügen haben, konkret beschreiben. Die Konversationsmaximen haben demnach Gebotscharakter.

192 Grice, H. Paul: „Logik und Konversation.“ In: Handlung, Kommunikation, Bedeutung. Hg. v. Georg Meggle. Frankfurt 1979, S. 248

193 Linke/Nussbaumer/Portmann 1996, S. 197

194 Linke/Nussbaumer/Portmann 1996, S. 196 


\begin{tabular}{|c|c|c|}
\hline $\begin{array}{c}\text { Konversationsma- } \\
\text { ximen }\end{array}$ & $\begin{array}{c}\text { Kommunikationsethische } \\
\text { Prinzipien } \\
\text { nach GRICE (1979) } \\
\end{array}$ & $\begin{array}{l}\text { Strategische Gebote } \\
\text { der Parteiräson nach } \\
\text { KLEIN (1996b) } \\
\end{array}$ \\
\hline Kooperationsprinzip & $\begin{array}{l}\text { Sei kooperativ und gestalte } \\
\text { deinen Beitrag zur Konversation } \\
\text { so, wie es der akzeptierte Zweck } \\
\text { oder die Ausrichtung des Ge- } \\
\text { sprächs, an dem du teilnimmst, } \\
\text { zum gegenwärtigen Zeitpunkt } \\
\text { erfordert. }\end{array}$ & $\begin{array}{l}\text { 1. Stelle die eigene } \\
\text { Position positiv dar! }\end{array}$ \\
\hline $\begin{array}{l}\text { Maxime der } \\
\text { Quantität }\end{array}$ & $\begin{array}{l}\text { Sage so viel wie nötig, aber nicht } \\
\text { zu viel: Gestalte deinen Beitrag } \\
\text { so informativ, wie es der gegen- } \\
\text { wärtige Zweck des Gesprächs } \\
\text { erfordert, jedoch nicht informa- } \\
\text { tiver als notwendig. }\end{array}$ & $\begin{array}{l}\text { 2. Demonstriere Leis- } \\
\text { tungsfähigkeit und } \\
\text { Durchsetzungskraft! }\end{array}$ \\
\hline Maxime der Qualität & $\begin{array}{l}\text { Versuche deinen Beitrag wahr } \\
\text { zu gestalten: Sage nichts, was du } \\
\text { für falsch hältst und sage nichts, } \\
\text { wofür du keine hinreichenden } \\
\text { Anhaltspunkte hast, oder signa- } \\
\text { lisiere, welchen Grad der Wahr- } \\
\text { scheinlichkeit das gesagte hat. }\end{array}$ & $\begin{array}{l}\text { 3. Stelle die gegnerische } \\
\text { Position als ablehnens- } \\
\text { wert dar! }\end{array}$ \\
\hline $\begin{array}{l}\text { Maxime der } \\
\text { Relevanz }\end{array}$ & $\begin{array}{l}\text { Sei relevant und sprich zum } \\
\text { Sachverhalt des Gesprächs. }\end{array}$ & $\begin{array}{l}\text { 4. Mache dir durch } \\
\text { deine Rede möglichst } \\
\text { wenig Gegner in rele- } \\
\text { vanten Gruppen! }\end{array}$ \\
\hline $\begin{array}{l}\text { Maxime der } \\
\text { Modalität } \\
\text { (Art und Weise) }\end{array}$ & $\begin{array}{l}\text { Gestalte deinen Beitrag in an- } \\
\text { gemessener Art und Weise und } \\
\text { so klar wie nötig: Vermeide } \\
\text { Unklarheit im Ausdruck, Mehr- } \\
\text { deutigkeiten, vermeide unnötige } \\
\text { Weitschweifigkeit und halte die } \\
\text { richtige Reihenfolge ein. }\end{array}$ & $\begin{array}{l}\text { 5. Halte dir Operations- } \\
\text { spielräume offen - auch } \\
\text { wenn du dich festlegen } \\
\text { mußt! }\end{array}$ \\
\hline
\end{tabular}

Die oben dargestellten Konversationsmaximen können als Fundamentalgebote einer gut funktionierenden (d.h. verständlichen) Kommunikation betrachtet werden; die Gegenüberstellung mit den strategischen Geboten der Parteien erhebt nicht den Anspruch einer direkten Entsprechung, sondern soll vielmehr aufzeigen, dass zwischen den Parteien in der politischen Kommunikation aufgrund des harten Konkurrenzkampfes die Maximen durch machtpolitische Handlungsgebote ersetzt werden. Die strategischen Gebote repräsentieren die Richtigkeit (1.), die

\footnotetext{
195 Grice 1979, S. 245ff.

${ }^{196}$ Klein 1996b, S. 10
} 
Kompetenz (2.) und Konsequenz (5.) in Bezug auf das eigene Handeln der politischen Akteure; die Gebote (3.) und (4.) „regulieren das öffentliche Verhalten der Politiker gegenüber ihren wichtigsten parteiexternen Adressaten: den Bürgern und den politischen Konkurrenten [...]. ${ }^{197}$ Dennoch werden sowohl sprachliche als auch schriftliche Äußerungen in der politischen Sprachverwendung an den Maximen von GRICE (1979) gemessen und bei deren Übertretung als polemisch, unfair, nichtssagend oder unglaubwürdig eingestuft.

Hinsichtlich der bereits dargestellten Makroverfahren sind die strategischen Gebote (1.) und (2.) dem LEGITIMIEREN der eigenen Partei zuzuordnen, während das Gebot (3.) das DELEGITIMIEREN der gegnerischen Position zum Ziel hat und ebenso dem Makroverfahren POLEMISIEREN zuzuordnen ist. Die Gebote (1.) und (2.) finden ihre Entsprechung im oben dargestellten Kapitel Anspruch auf Erfüllbarkeit; das strategische Gebot (3.) wurde in den beiden vorangegangenen Kapiteln Anklagen/Vorwürfe und Expliziter Gegnerbezug behandelt. Das Gebot (4.) wird erneut aufgegriffen, wenn es an späterer Stelle dieser Untersuchung um die unterschiedlichen Adressatengruppen und deren Rezeption der Parteiprogramme geht; das strategische Gebot, sich als Sprecher nicht festzulegen, auch wenn es notwendig ist und dadurch inhaltsarme Aussagen zu formulieren, wird in den nachfolgenden Kapiteln Kalkulierte Ambivalenz, Kompromiss-Formulierungen sowie insbesondere im Kapitel Leerformeln ausführlich beleuchtet. Die Kapitel stellen mit ihrer Thematik auch konkrete Auswirkungen der oben dargestellten Mehrfachadressierung dar.

\subsubsection{Bewusste Nicht-Erwähnungen}

Wenn bestimmte Tatsachen in einem politischen Text nicht erwähnt werden, obwohl diese von der Sache her unbedingt erwähnt werden müssten, dann geht mit dieser Nicht-Erwähnung eine bestimmte Funktion einher. Die Verfasser eines Textes können mit diesem sprachlichen Mittel zeigen, dass sie sich von dieser Sache distanzieren, eben weil sie diese nicht erwähnen. Der sachliche Inhalt bleibt als Gegenstand allerdings erhalten, was gegen die Auffassung spricht, dass das Nicht-Erwähnte auch nicht Bestandteil des Textes sein kann. Diese Annahme resultiert aus folgender These:

Wenn etwas, das bisher einen zentralen Bestandteil eigener Politik oder Tradition ausgemacht hat, nicht erwähnt wird, soll dies als ostentatives Zeichen stärkster Distanzierung verstanden werden. ${ }^{198}$

\footnotetext{
197 Klein 1996b, S. 11

198 Klein 1996a, S. 208
} 
In diesem Sinne lässt sich auch hinsichtlich des Godesberger Programms die bewusste Vermeidung einschlägiger Begriffe attestieren. So heißt es in Äußerung (a):

(a) Der demokratische Sozialismus, der in Europa christlicher Ethik, im Humanis-mus und in der klassischen Philosophie verwurzelt ist, will keine letzten Wahrheiten verkünden - [...]. (Godesberger Programm 1959, S. 69)

(b) Der Demokratische Sozialismus in Europa hat seine geistigen Wurzeln im Christentum und in der humanistischen Philosophie, in der Aufklärung, in Marxscher Geschichts- und Gesellschaftslehre und in den Erfahrungen der Arbeiterbewegung. (SPD 1989, S. 10)

Mit der historischen Darstellung in Äußerung (a) stellt sich die SPD allerdings eine andere Identität aus. Zwar gibt das Godesberger Programm mit den oben zitierten Punkten keine falschen Tatsachen an, es verschweigt aber die eigentliche Genese der SPD als Protestbewegung der Arbeiterklasse; , es läßt [...] etwas Wichtiges aus, das offenbar jetzt stört, wie eine böse Erinnerung. Etwas, das offenbar jetzt nur noch peinlich ist und das den Leser nur auf falsche Gedanken bringen könnte. " ${ }^{199}$ Dieses bewusst Verschwiegene sind der Name Marx sowie die Ausdrücke marxistisch und Marxismus. Der oben zitierten These folgend werden diese Ausdrücke als "ostentative Zeichen“ gewertet; offensichtlich oder augenscheinlich können sie als Nicht-Erwähnungen jedoch nur für den Teil der Rezipienten sein, der über ein gefestigtes geschichtliches Wissen verfügt und insbesondere über die Historie der SPD als Partei Kenntnis hat. Somit bezieht sich die oben formulierte These speziell auf fachkundige Empfänger. Während nicht-fachkundige Rezipienten durch die Auslassung einfach nicht aufmerksam gemacht werden, wird an fachkundige Rezipienten ein starkes „ostentatives“ Zeichen der Distanzierung übermittelt. Die Gesellschaftstheorie von Marx ließ sich offenbar mit der wirtschaftlichen und gesellschaftlichen Situation in der BRD während der 1960er Jahre (Wirtschaftsaufschwung, Kontroverse der Wiederbewaffnung, Modell der Marktwirtschaft, Wohlstand) nicht vereinbaren; die SPD verabschiedet sich von der traditionellen und revolutionären Ideenwelt und öffnet sich für andere - weniger radikale - theoretische Begründungen (die Wendung zur Volkspartei geht mit dieser Entwicklung einher).

Die Äußerung (b) zeigt anschaulich auf, dass die gesellschaftlichen Rahmenbedingungen einen immensen Einfluss auf die Konzeption eines Parteiprogramms haben. Die Anlehnung der Formulierungen des Programms von 1989 an die des Godesberger Programms sind signifikant, es

199 Hermanns, Fritz: „Deontische Tautologien.“ In: Politische Semantik. Beiträge zur politischen Sprachverwendung. Hg. v. Josef Klein. Opladen 1989, S. 86 
bestand nun - aufgrund der veränderten gesellschaftlichen Rahmenbedingungen - allerdings keine Veranlassung mehr für die SPD, als Zeichen der Distanzierung auf den Ausdruck Marx zu verzichten. Durch die explizite Erwähnung bekennt sich die SPD nun zu ihrer Tradition bzw. zu ihrer Entstehungsgeschichte.

Zwei weitere nahezu wörtlich übereinstimmende Textstellen beider SPD-Programme sollen nachfolgend gegenübergestellt werden. Auffällig ist, dass die SPD im Jahre 1989 auf die Ausdrücke als dienendes Glied der Gemeinschaft sowie auf Menschheit verzichtet; weiterhin wird Sozialisten durch Sozialdemokratie ersetzt.

\begin{tabular}{|c|c|}
\hline r Programm 1959 & Grundsatzprogramm der SPD 1989 \\
\hline $\begin{array}{l}\text { Die Sozialisten erstreben eine Gesellschaft, } \\
\text { in der jeder Mensch seine Persönlichkeit in } \\
\text { Freiheit entfalten und als dienendes Glied } \\
\text { der Gemeinschaft verantwortlich am poli- } \\
\text { tischen, wirtschaftlichen und kulturellen } \\
\text { Leben der Menschheit mitwirken kann. } \\
\text { (S. 69) }\end{array}$ & $\begin{array}{l}\text { Die Sozialdemokratie erstrebt eine Gesell- } \\
\text { schaft, in der jeder Mensch seine Persön- } \\
\text { lichkeit in Freiheit entfalten und verant- } \\
\text { wortlich am politischen, wirtschaftlichen } \\
\text { und kulturellen Leben mitwirken kann. } \\
\text { (S. 12) }\end{array}$ \\
\hline
\end{tabular}

Abschließend sei angemerkt, dass das ostentative Ignorieren im direkten politischen Meinungsstreit auch als Mittel der Abwertung eingesetzt werden kann.

\subsubsection{Kompromiss-Formulierungen}

In einer beliebigen politischen Debatte stehen kontroverse Themen zur Diskussion, die von den jeweiligen Diskussionsteilnehmern auf unterschiedliche Art und Weise interpretiert und vertreten werden. In manchen Fällen ist es jedoch gemeinsames Ziel aller am Streit Beteiligten, letztlich zu einem Ergebnis zu kommen. Eine Möglichkeit kann dabei das Finden und Eingehen eines Kompromisses sein.

Eine Übereinkunft auf Basis eines Kompromisses setzt eine Verständigung sowie beiderseitiges Nachgeben voraus. Die nachfolgend zitierte Textpassage des Godesberger Programms verdeutlicht anschaulich, wie die jeweiligen Seiten mit ihren Positionen Zugeständnisse machen, um zu einem Ergebnis zu gelangen. Die Kontroverse bezieht sich auf die Massenmedien als Institution und auf das Reizwort öffentlich-rechtlich:

(26) (a) Presse, Rundfunk, Fernsehen und Film erfüllen öffentliche Aufgaben. Sie müssen in Freiheit und Unabhängigkeit überall und unbehindert Informationen sammeln, bearbeiten, verbreiten und unter eigener Verantwortung Meinungen bilden und aussprechen dürfen. 
(b) Rundfunk und Fernsehen müssen ihren öffentlichrechtlichen Charakter behalten. Sie müssen freiheitlich-demokratisch geleitet und gegen Interessendruck gesichert sein. (Godesberger Programm 1959, S. 72)

Es stellt sich nun die Frage, worin in dieser Formulierung der Kompromiss liegt und welche Zugeständnisse von beiden Seiten gemacht werden müssen. Die Zugeständnisse von Presse, Rundfunk, Fernsehen und Film liegen in einer möglichen Einschränkung ihres Handlungsspielraums (b), wenn diese Institutionen Anstalten des öffentlichen Rechts bleiben und damit als verselbständigte Verwaltungsgebilde zur Erfüllung besonderer öffentlicher Aufgaben gelten. Die zentrale Aufgabe der öffentlich-rechtlichen Rundfunkanstalten ist die „Versorgung aller Bürgerinnen und Bürger mit Informationen, Bildung, Kultur und Unterhaltung"200; "die Berichterstattung soll umfassend, wahrheitsgetreu und sachlich sein."201 Solche Aufgaben werden vom Bundesverfassungsgericht als Fernsehurteile formuliert und gelten als Satzungen der Anstalten zur Vorbeugung der einseitigen politischen Ausrichtung ${ }^{202}$ und Kommerzialisierung; die unabhängige, freie und unbehinderte Informationsverarbeitung und deren Verbreitung in der Öffentlichkeit durch Journalisten ist damit aufgrund der Überwachung durch die Aufsichtsgremien und deren Programmauswahl eingeschränkt. Der Kompromiss der SPD oder der Parteien allgemein liegt in der Reduzierung der Einfußnahme auf das Mediengeschehen (a), obwohl Parteien durch Anregungen und Kritik versuchen, ein bestimmtes Programm zu beeinflussen. Die Massenmedien sind maßgeblich an der Werbung und Bekanntmachung einer Partei und deren Inhalte beteiligt und daher für diese von enormer Bedeutung. Es ist somit nicht verwunderlich, dass sich die Kontrollorgane aus Vertretern der Parteien zusammensetzen. Aus diesem Grund hält auch die SPD an dem öffentlich-rechtlichen Sys-

200 Massenmedien. In: Informationen zur politischen Bildung 260. Hg. v. Bundeszentrale für politische Bildung. Bonn 1998, S. 28

201 Massenmedien 1998, S. 32

202 Das Ziel der Informationsverbreitung über die Massenmedien, insbesondere der öffentlich-rechtlichen Rundfunkanstalten, ist eine allumfassende Darstellung aller bedeutender Ereignisse. Dies soll nach Möglichkeit ohne Wertung der Medien geschehen, jedoch ist die Darstellung von Medienereignissen allein durch deren Auswahl und Formulierung nicht als objektiv zu bewerten. Unterschieden wird prinzipiell zwischen der so genannten binnenpluralistischen Struktur, nach der jeder Veranstalter für ein vielfältiges Programm sorgen muss, und der so genannten außenpluralistischen Struktur, nach der das Gesamtangebot der Programme aller Veranstalter die bestehende Meinungsvielfalt widerspiegeln soll. Die öffentlich-rechtlichen Rundfunkanstalten orientieren sich demzufolge an der binnenpluralistischen Struktur. 
tem fest, nicht jedoch ohne ihre Ablehnung gegenüber einem Interessendruck jeglicher Art deutlich zu formulieren.

Eine weitere Kontroverse des Parteiprogramms bezieht sich auf die Wirtschaftspolitik:

(c) Die Wirtschaftspolitik muß auf der Grundlage einer stabilen Währung die Voll-beschäftigung sichern, die volkswirtschaftliche Produktivität steigern und den allgemeinen Wohlstand erhöhen.

(d) Um alle Menschen am steigenden Wohlstand zu beteiligen, muß die Wirtschaft en ständigen Strukturveränderungen planmäßig angepaßt werden, damit eine ausgeglichene Wirtschaftsentwicklung erreicht wird. (Godesberger Programm 1959, S. 74)

(e) Zur Erreichung der Vollbeschäftigung müssen alle geeigneten Mittel ausgeschöpft werden. Maßnahmen zur Arbeitszeitverkürzung müssen in Einklang stehen mit dem wirtschaftlichen Wachstum und der Vollbeschäftigung. (CDU 1978, S. 144)

In der oben zitierten Textpassage (c) und (d) des Godesberger Programms ist der Konflikt mit den Reizwörtern Vollbeschäftigung und Wohlstand sowie ständige Strukturveränderungen verknüpft. Zwischen Erwähnen und Nicht-Erwähnen solcher Reizwörter gibt es in einer Formulierung keinen Mittelweg. Trotz dieser Schwierigkeit ist das Finden eines Kompromisses von großer Wichtigkeit, "denn Konflikt um Reizwörter sind stark prestigebesetzt und für die Verlierer mit hohen Imageeinbußen verbunden.“203 Die SPD kann es sich aus strategischen Gründen im Hinblick auf den hohen Anteil der Arbeiterschaft als Adressatengruppe nicht erlauben auf den Ausdruck Vollbeschäftigung zu verzichten. Wie auch immer die konkreten Realisierungen der planmäßigen Anpassung aufgrund der ständigen Strukturveränderungen aussehen mögen, sie sind in der Formulierung (b) ein Mittel, die Forderung unter (a) einzuschränken; hinter beiden Ausdrücken kann sich auch ein betrieblicher Stellenabbau verbergen, der mit der Sicherung der Arbeit und mit dem Erhalt und der Rettung der anderen Arbeitsplätze gerechtfertigt wird. Die SPD bekennt sich im Godesberger Programm zur Marktwirtschaft und aufgrund des allgemein einsetzenden „Wirtschaftswunders" und dem einhergehenden Wohlstand des Großteils der deutschen Bundesbürger kommt die SPD ebenso wenig umhin, den Ausdruck Wohlstand in ihr Programm aufzunehmen. Die Formulierung unter (b) beschreibt mögliche Eingriffe des Staates in die Wirtschaftsabläufe und bezieht sich unter Berücksichtigung von (a) sowohl auf den Wirtschaftsflügel als auch auf den linken Flügen innerhalb der Partei und innerhalb der Bevölkerung, die der sozialistischen Tradition gemäß eventuelle staatliche Eingriffe in Wirtschaftsabläufe befürwortet. Die Erwähnung eines Reizwortes in

203 Klein 1996a, S. 203 
einem Parteiprogramm lässt sich folgendermaßen begründen: „Aufnahme oder Nicht-Aufnahme eines umkämpften Reizwortes in ein Parteiprogramm ist ein Zeichen dafür, wer im innerparteilichen Konflikt gewonnen hat. “204

Der oben zitierten Regel folgend sind die Formulierungen (a) und (b) aufgrund der Erwähnung der dargestellten Reizwörter als Kompromiss $\mathrm{zu}$ verstehen, da dadurch auf beide Flügel gleichermaßen Bezug genommen wird. Darüber hinaus werden die Reizwörter direkt in die unterschiedlichen Forderungen eingewoben; aus der Sprachverwendung lässt sich somit nachvollziehen, ob ein Reizwort textuell entschärft wird oder ob einem solchen sprachlichen Ausdruck ein hohes Maß an Bedeutung eingeräumt wird. Auf diese Weise muss auch die Äußerung (c) der CDU verstanden werden. Was primär als Handlungsrichtlinie der Partei mit dem Ziel der Vollbeschäftigung scheint ist tatsächlich als Kompromiss nach harten Auseinandersetzungen zwischen unterschiedlichen Flügeln innerhalb der CDU zu betrachten. Im Mittelpunkt steht dabei die Diskussion um das Schlagwort Arbeitszeitverkürzung; dessen Aufnahme (der oben zitierten Regel folgend) müsste daher als Zeichen zu bewerten sein, dass einer der beiden Flügel den innerparteilichen Konflikt gewonnen hat. Ausschlaggebend für die Interpretation der Äußerung (d) als Kompromiss ist jedoch auch hier die textuelle Entschärfung aufgrund der Position des Schlagwortes. Dieser Ermessensspielraum resultiert aus folgender sprachhandlungstheoretischer Regel:

Wenn ein Reizwort nicht an zentraler Stelle und nicht im Modus der Forderung verwendet wird, sondern in unauffälliger Position und im Modus der Geltungsbeschränkung, dann wird damit gezeigt, daß dem vom Reizwort bezeichneten Konzept nur ein geringer Stellenwert beigemessen wird. ${ }^{205}$

In der politischen Sprachverwendung ist allerdings auch eine Form der Übereinkunft zweier Positionen ohne Abstriche denkbar; diese Form wird als Programmformulierung durch den Terminus „Kalkulierte Ambivalenz ${ }^{\prime 206}$ verdeutlicht.

\subsubsection{Kalkulierte Ambivalenz}

Die doppelwertige oder doppeldeutige Formulierung nach dem Prinzip der kalkulierten Ambivalenz dient dazu, durch die Erwähnung und gleichzeitiger Bejahung jeder der gegenüber stehenden unvereinbaren Positionen diese als vereinbar erscheinen zu lassen. Dadurch müssen auf

$\begin{array}{ll}204 & \text { Klein 1996a, S. } 204 \\ 205 & \text { Klein 1996a, S. } 204 \\ 206 & \text { Klein 1996a, S. } 206\end{array}$ 
beiden Seiten keine Zugeständnisse gemacht werden und der Gesichtsverlust der konfligierenden Positionen kann durch die ambivalente Formulierung jeweils so gering wie möglich gehalten werden. Die Unvereinbarkeit der Positionen wird dabei nicht thematisiert. Diese Widersprüchlichkeit basiert auf folgender sprachlichen Regel:

Wenn zwei Gruppen, deren Positionen miteinander unvereinbar sind und zwischen denen eine Kompromißlinie nicht formuliert werden kann, beide für die Partei besonders wichtig sind, ist das Verhältnis der Partei zu beiden positiv zu formulieren, ohne auf deren Unvereinbarkeit näher einzugehen. ${ }^{207}$

Im Godesberger Programm finden sich folgende Beispiele einer strategisch ambivalenten Formulierung im Sinne der oben zitierten Regel:

(27) (a) Sie [die SPD] bejaht die Landesverteidigung. [...] Die Sozialdemokratische Partei fordert die völkerrechtliche Ächtung der Massenvernichtungsmittel auf der ganzen Welt. Die Bundesrepublik Deutschland darf atomare und andere Massenvernichtungsmittel weder herstellen noch verwenden. [...] Die Sozialdemokratische Partei Deutschlands stellt sich schützend vor jeden Bürger, der aus Gewissensgründen den Dienst mit der Waffe oder an Massenvernichtungsmitteln verweigert. (Godesberger Programm 1959, S. 73)

(b) Wir bejahen die Bundeswehr und die Wehrpflicht. [...] Wir achten das Engagement von Pazifisten, die für die Utopie einer gewaltfreien Völkergemeinschaft einstehen. Sie haben einen legitimen Platz in der SPD. Wir garantieren das Grundrecht auf Kriegsdienstverweigerung. (SPD 1989, S. 16)

Die SPD führt die Notwendigkeit der Landesverteidigung an (a). Um diese Aufgabe zu erfüllen ist die Aufstellung einer Armee (Bundeswehr) erforderlich, d.h. die Regierung ist auf Soldaten angewiesen. Die Landesverteidigung kann ohne Soldaten und deren Dienst an der Waffe nicht gewährleistet werden. Die konfligierende Position ist die Verweigerung des Dienstes an der Waffe aus Gewissensgründen (d); die Berufung auf dieses Verweigerungsrecht wird grundsätzlich jedermann ermöglicht. Würde nun jedermann dieses Recht in Anspruch nehmen, dann wäre die Landesverteidigung überhaupt nicht möglich; die Formulierung unter (a) setzt folglich voraus, dass niemals alle wehrpflichtigen Personen diesen Dienst verweigern. Beide Positionen - Dienst und Verweigerung sind unvereinbar, können außerdem nicht durch einen Kompromiss gelöst werden und sind dennoch positiv formuliert. Ein weiterer Widerspruch liegt in der Auffassung, dass Pazifisten einen Platz in einer Partei haben sollen, die die Landesverteidigung bejaht. Weiterhin spricht sich die SPD gegen Massenvernichtungsmittel allgemein und insbesondere gegen deren Herstellung und Verwendung aus (a). Da der Dienst an

207 Klein 1996a, S. 206 
Massenvernichtungsmitteln lediglich dann nicht erforderlich ist, wenn eine wehrpflichtige Person den Dienst verweigert, impliziert diese Formulierung, dass der Dienst an Massenvernichtungsmitteln normalerweise üblich ist im Sinne der Ausübung eines Soldaten (a). Problematisch an dieser Analyse ist die unklare Bedeutung des Ausdrucks Massenvernichtungsmittel, der als "Sammelbezeichnung namentlich für Kernwaffen und ABC-Waffen“208 gebräuchlich ist. In Äußerung (a) wird der Ausdruck lediglich von atomaren Waffen zwar abgegrenzt, er bleibt als Begrifflichkeit jedoch verschwommen.

(28) (a) Freie Konsumwahl und freie Arbeitsplatzwahl sind entscheidende Grundlagen, freier Wettbewerb und freie Unternehmerinitiative sind wichtige Elemente sozialdemokratischer Wirtschaftspolitik. $[\ldots]$

(b) Leistungsfähige mittlere und kleine Unternehmen sind zu stärken, damit sie die wirtschaftliche Auseinandersetzung mit den Großunternehmen bestehen können.

(c) Wettbewerb durch öffentliche Unternehmen ist ein entscheidendes Mittel zur Verhütung privater Marktbeherrschung. [...]

(d) Sie werden dort zur Notwendigkeit, wo aus natürlichen oder technischen Gründen unerläßliche Leistungen für die Allgemeinheit nur unter Ausschluß eines Wettbewerbs wirtschaftlich vernünftig erbracht werden können. (Godesberger Programm 1959, S. 74/75)

Der freie Wettbewerb und die freie Unternehmerinitiative sind wichtige Voraussetzungen für den Wirtschaftsablauf, die die SPD unter Kapitel Stetiger Wirtschaftsaufschwung anführt (a). Einige Sätze später unter Kapitel Eigentum und Macht wird der freie Wettbewerb allerdings eingeschränkt (b) und (c). Dieses Handeln ist durchaus legitim, die Begründung liegt in der Verhinderung der Bildung von Kartellen und das Kräfteungleichgewicht zwischen Großunternehmen einerseits sowie kleinen und mittleren Unternehmen andererseits. Aus diesem Grund befürwortet die SPD ein staatliches Eingreifen zugunsten der Interessen der Allgemeinheit; die SPD behält sich darüber hinaus vor, in bestimmten wirtschaftlichen Situationen den Wettbewerb komplett auszuschließen (d). Jedoch macht diese Feststellung allein die eigentliche Problematik hinsichtlich der Sprachhandlungen nicht deutlich: Wie bereits beim vorangegangenen Beispiel sind auch hier beide Positionen unvereinbar und ein Kompromiss wenig wahrscheinlich. Die Lösung auf politolinguistischer Ebene liegt auch hier in der positiven Erwähnung der unvereinbaren Positionen.

208 Weber-Fas, Rudolf: Das kleine Staatslexikon. Stuttgart 2000, S. 298 
(29) (a) Die Sozialdemokratische Partei will Lebensbedingungen schaffen, unter denen alle Menschen in freier Entschließung aus steigendem Einkommen eigenes Vermögen bilden können. [...]

(b) Es ist ein Zeichen unserer Zeit, daß sich das private Wohlleben privilegierter Schichten schrankenlos entfaltet, während wichtige Gemeinschaftsaufgaben, vor allem Wissenschaft, Forschung und Erziehung, in einer Weise vernachlässigt werden, die einer Kulturnation unwürdig ist. (Godesberger Programm 1959, S. 77)

Die SPD ist der Auffassung, dass die Marktwirtschaft an sich keine gerechte Einkommens- und Vermögensverteilung gewährleistet und die soziale Komponente vernachlässigt. Die Betonung liegt hierbei auf gerecht. Die SPD zeichnet unter (b) ein Bild der sozialen Marktwirtschaft, in der die Regierung in das Marktgeschehen eingreift, und nicht das der freien Marktwirtschaft: „Die Lohn- und Gehaltspolitik ist ein geeignetes und notwendiges Mittel, um Einkommen und Vermögen gerechter zu machen" (Godesberger Programm 1959, S. 77). Problematisch wird die Formulierung (a) hinsichtlich des Wortes alle, da jedermann laut SPD berechtigt ist, Kapital zu bilden, d.h. privates Vermögen aufzubauen. Damit spricht die SPD eine Gesellschaftsschicht als Adressatengruppe an, die finanziell und aufgrund eines positiven beruflichen Werdegangs in der Lage ist, Vermögen zu bilden. Die SPD spricht mit der Formulierung (b) jedoch gleichermaßen diejenige Gesellschaftsschicht an, die sich nicht in der Lage befindet, Kapital anhäufen zu können; an diese Adressatengruppe gerichtet spricht die SPD von schrankenloser Entfaltung und der Vernachlässigung anderer wichtiger Bereiche in der Gesellschaft. Auch in diesem Fall handelt es sich um zwei unvereinbare Positionen, die jedoch durch die Erwähnung an unterschiedlichen Stellen des Kapitels Einkommens- und Vermögensverteilung gleichermaßen bedient werden. Aufgrund der Verwendung des Ausdrucks schrankenlos stellt sich allerdings die Frage, ob die Bedingung der positiven Formulierung eingehalten wurde.

(30) (a) Wissenschaftliche Forschung und Lehre müssen frei sein.

Ihre Ergebnisse sind der Öffentlichkeit zugänglich zu machen. [...]

(b) Der Staat hat Vorsorge zu treffen, daß Forschungsergebnisse nicht zum Schaden der Menschheit mißbraucht werden. (Godesberger Programm 1959, S. 83)

Die SPD fordert unter (a), dass Forschungsergebnisse öffentlich gemacht werden sollen. Diese Formulierung ist prinzipiell unvereinbar mit der darüber hinaus gestellten Forderung, dass der Staat die Forschungsergebnisse auf ihre Gefahr und die Möglichkeit des Missbrauchs hin überprüft. Diese Tatsache an sich ist möglicherweise positiv zu bewerten, bietet sie doch einen möglichen Schutz jeden einzelnen Mitglieds in der Gesellschaft. Es heißt allerdings auch, dass diese Forschungsergebnisse 
in einem solchen Fall der Öffentlichkeit nicht mehr frei zugänglich sind, was die Aussage unter (a) gewissermaßen aushebelt.

(31) (a) Freiheit und Unabhängigkeit der Hochschulen bleiben unberührt.

(b) Die Hochschulen können aber nicht isoliert von der übrigen Lebenswirklichkeit bestehen und sollten darum mit anderen Institutionen der demokratischen Gesellschaft, insbesondere mit den Einrichtungen der Erwachsenenbildung, zusammenarbeiten. (Godesberger Programm 1959, S. 83)

Die SPD versichert in Äußerung (a), dass die Hochschulen unberührt bleiben. Diese Position wird jedoch direkt im Anschluss unter (b) eingeschränkt; die Einschränkung wird mit dem Wort aber signalisiert. Beide Positionen sind unvereinbar: Wenn die Hochschulen nicht isoliert bestehen bleiben können, dann sind sie folglich auch nicht unberührt im Sinne von unbeeinflusst oder eigenständig. Sie sind stattdessen zur Kooperation mit anderen Institutionen der demokratischen Gesellschaft (diese werden nicht spezifiziert) und den Einrichtungen der Erwachsenenbildung aufgerufen. Möglicherweise will die SPD mit der Formulierung unter (a) deutlich machen, dass Bildung unabhängig von der parteispezifischen Weltanschauung ermöglicht werden soll und die Hochschulen nicht als Plattform der parteiideologischen Inhalte fungieren. In den Formulierungen (a) und (b) stehen jedenfalls zwei Aussagen gegeneinander, die sich widersprechen und auch im Sinne der oben zitierten Regel nicht durch irgendeinen Kompromiss zu lösen sind.

Die kalkuliert ambivalente Bedeutung ist jedoch nicht auf Textpassagen oder die Gegenüberstellung von Sätzen beschränkt; auch einzelne Termini können begrifflich mehrdeutig sein. Ein Beispiel für eine „ideologische Mehrdeutigkeit" ${ }^{\prime 209}$ ist die strategische Nutzung des Eigenbegriffs im Godesberger Programm der SPD: demokratischer Sozialismus. Die SPD nimmt im Godesberger Programm mit der Wendung zur Volkspartei dezidiert Abschied von den ursprünglichen Merkmalen planwirtschaftlich, neutralistisch-pazifistisch und antikirchlich, obwohl der Ausdruck demokratischer Sozialismus beibehalten wird - sogar an zentraler Stelle der Präambel (vgl. Kapitel 2.1.4.4.1, Zeile 36). Somit werden drei Lesarten ermöglicht:

1. Für Leser, die das Programm, wörtlich' verstehen, ist der Begriff die Bezeichnung für die neue Politik. 2. Für traditionsbewusste Parteimitglieder mit geringem Programmverständnis signalisiert die Beibehaltung des Ausdrucks Kontinuität. Ihnen gegenüber handelt es sich um die Strategie, ein Wort als ,Vexierwort' zu verwenden, d.h. die Akzeptanz, die ein Begriff in seiner traditionellen Bedeutung genießt, zu 
nutzen, um hinter der Fassade des Ausdrucks möglichst unbemerkt eine Bedeutungsänderung vorzunehmen [...]. 3. Ideologisch links orientierten Mitgliedern bietet der Begriff aufgrund seiner ,linken' Konnotationen die Chance, trotz der programmatischen Neuorientierung auf die Reaktivierung der traditionellen Bedeutung hinzuarbeiten $[\ldots] \cdot{ }^{210}$

Im Zuge der Untersuchung der zitierten Textpassagen des Godesberger Programms wurde deutlich, dass die Formulierungen sich offensichtlich in starkem Maße an den Rezipienten orientieren. Die kalkuliert ambivalenten Formulierungen haben das Ziel, verschiedene Adressatengruppen mit unterschiedlichen Positionen anzusprechen und $\mathrm{zu}$ gewinnen. Es scheint daher lohnenswert, diese Adressatengruppen zu einem späteren Zeitpunkt genauer zu betrachten.

\subsubsection{Das Godesberger Programm und der Grundgesetztext - Gemeinsamkeiten und Unterschiede}

Grundsätzlich stimmen das Godesberger Programm und der Grundgesetztext insofern überein, als dass beide Texte in komprimierter Form und mit teilweise vage formulierten Aussagen eine gegenwärtige Gesellschaftsordnung abbilden, eine angestrebte Ordnung fordern oder Grundprinzipien gesellschaftlichen Verhaltens vorschreiben (der komprimierte Satzbau wird hauptsächlich durch die nominalisierte Wortbildung hervorgerufen, auf die in einem späteren Kapitel noch ausführlich eingegangen werden soll). Bei einer eingehenden Betrachtung der Aussagen des Godesberger Programms fällt bei einigen Formulierungen eine deutliche Nähe zum Grundgesetztext auf; auf diese Nähe berufen sich sowohl die SPD im Godesberger Programm von 1959 in Äußerung (a) sowie die CDU im Jahre 1978 in Äußerung (b) wörtlich:

(32) (a) Die Sozialdemokratische Partei Deutschlands lebt und wirkt im ganzen deutschen Volke. Sie steht zum Grundgesetz der Bundesrepublik Deutschland. (Godesberger Programm 1959, S. 71)

(b) Das Grundgesetz der Bundesrepublik Deutschland ist Grundlage für unser Zusammenleben in Freiheit, Solidarität und Gerechtigkeit. Diese Grundwerte lassen sich nur in einem demokratischen und sozialen Rechtsstaat verwirklichen, wie er dem Auftrag des Grundgesetzes entspricht. Das Grundgesetz der Bundesrepublik Deutschland beruht auf einem unantastbaren Grundbestand gemeinsamer Wertüberzeugungen. (CDU 1978, S. 155)

(c) Wie auch immer wir die Würde des Menschen begründen, sie ist Ausgangs- und Zielpunkt unseres Handelns. Für uns alle gilt der Satz, mit dem die Vereinten Nationen ihre Erklärung der Menschenrechte einleiten: ,Menschen sind frei und gleich an Würde und Rechten

$210 \quad$ Klein 1998, S. 387 
geboren. Sie sind mit Vernunft und Gewissen begabt und sollen einander im Geiste der Brüderlichkeit begegnen.' (SPD 1989, S. 10)

Die Äußerung (c) verdeutlicht den Einfluss der Aussagen und Inhalte von Verfassungstexten auf die Konzeption eines Parteiprogramms. So reicht auch der Globalverweis auf das Grundgesetz in (a) der SPD offensichtlich nicht aus, vielmehr wird durch häufige direkte und indirekte Anlehnung des Godesberger Programms an den Grundgesetztext der Bezug zu diesem betont. Im Folgenden sollen nun signifikante Textstellen des Grundsatzprogramms mit den sowohl explizit als auch implizit korrespondierenden Formen des Grundgesetzes exemplarisch gegenübergestellt werden:

\begin{tabular}{|c|c|c|}
\hline Thema & Godesberger Programm & Grundgeset $^{211}$ \\
\hline $\begin{array}{l}\text { Freie Entfaltung } \\
\text { der Persönlich- } \\
\text { keit }\end{array}$ & $\begin{array}{l}\text { Die Sozialisten erstreben eine } \\
\text { Gesellschaft, in der jeder } \\
\text { Mensch seine Persönlichkeit in } \\
\text { Freiheit entfalten }[\ldots] \text { kann. } \\
\text { (S. 69) }\end{array}$ & $\begin{array}{l}\text { Jeder hat das Recht auf die } \\
\text { freie Entfaltung seiner Persön- } \\
\text { lichkeit, }[\ldots] .(\text { Art. } 2 ; 1 \text { ) }\end{array}$ \\
\hline $\begin{array}{l}\text { Achtung der } \\
\text { Mitmenschen }\end{array}$ & $\begin{array}{l}\text { Freiheit und Gerechtigkeit be- } \\
\text { dingen einander. Denn die } \\
\text { Würde des Menschen liegt im } \\
\text { Anspruch auf Selbstverantwor- } \\
\text { tung ebenso wie in der Aner- } \\
\text { kennung des Rechtes seiner } \\
\text { Mitmenschen, ihre Persönlich- } \\
\text { keit zu entwickeln und an der } \\
\text { Gestaltung der Gesellschaft } \\
\text { gleichberechtigt mitzuwirken. } \\
\text { (S.69) }\end{array}$ & $\begin{array}{l}{[\ldots] \text {, soweit er nicht die Rechte }} \\
\text { anderer verletzt und nicht } \\
\text { gegen die verfassungsmäßige } \\
\text { Ordnung oder das Sittengesetz } \\
\text { verstößt. (Art. } 2 ; 1 \text { ) }\end{array}$ \\
\hline $\begin{array}{l}\text { Gleichberechti- } \\
\text { gung von Mann } \\
\text { und Frau }\end{array}$ & $\begin{array}{l}\text { Die Gleichberechtigung der Frau } \\
\text { muß rechtlich, sozial und wirt- } \\
\text { schaftlich verwirklicht werden. } \\
\text { Der Frau müssen die gleichen } \\
\text { Möglichkeiten für Erziehung } \\
\text { und Ausbildung, für Berufs- } \\
\text { wahl, Berufsausübung und } \\
\text { Entlohnung geboten werden wie } \\
\text { dem Mann. (S. 80) }\end{array}$ & $\begin{array}{l}\text { Männer und Frauen sind } \\
\text { gleichberechtigt. (Art. 3; 2) }\end{array}$ \\
\hline
\end{tabular}

211 Verfassung des Landes Hessen und Grundgesetz für die Bundesrepublik Deutschland. Mit einer Einführung und einem Anhang: Allgemeine Erklärung der Menschenrechte - Konvention zum Schutze der Menschenrechte und Grundfreiheiten (Auszug) - Charta der Vereinten Nationen. Hg. v. Georg August Zinn und Erwin Stein. Bad Homburg vor der Höhe ${ }^{45} 1992$, S. 162-220 


\begin{tabular}{|c|c|c|}
\hline $\begin{array}{l}\text { Keine Benach- } \\
\text { teiligung, } \\
\text { Bevorzugung } \\
\text { oder sonstige } \\
\text { Einmischung } \\
\text { des Staates }\end{array}$ & $\begin{array}{l}\text { Der demokratische Sozialismus } \\
{[\ldots] \text { will keine letzten Wahrhei- }} \\
\text { ten verkünden [...] aus der } \\
\text { Achtung vor den Glaubensent- } \\
\text { scheidungen des Menschen, } \\
\text { über deren Inhalt weder eine } \\
\text { politische Partei noch der Staat } \\
\text { zu bestimmen haben. (S. 69) }\end{array}$ & $\begin{array}{l}\text { Niemand darf wegen seines } \\
\text { Geschlechtes, seiner Abstam- } \\
\text { mung, seiner Rasse, seiner } \\
\text { Sprache, seiner Heimat und } \\
\text { Herkunft, seines Glaubens, } \\
\text { seiner religiösen oder politi- } \\
\text { schen Anschauungen benach- } \\
\text { teiligt oder bevorzugt werden. } \\
(\text { Art. 3; 3) }\end{array}$ \\
\hline $\begin{array}{l}\text { Glaubens-, } \\
\text { Bekenntnis- und } \\
\text { Gewissensfrei- } \\
\text { heit }\end{array}$ & $\begin{array}{l}\text { Der Staat ist verpflichtet, die } \\
\text { Freiheit des Glaubens und des } \\
\text { Gewissens zu sichern. (S. 71) } \\
\text { Freiheit des Denkens, des Glau- } \\
\text { bens und des Gewissens [...] } \\
\text { sind zu sichern. (S. 81) }\end{array}$ & $\begin{array}{l}\text { Die Freiheit des Glaubens, des } \\
\text { Gewissens und die Freiheit } \\
\text { des religiösen und weltan- } \\
\text { schaulichen Bekenntnisses } \\
\text { sind unverletzlich. (Art. 4; } 1 \text { ) } \\
\text { Die ungestörte Religionsaus- } \\
\text { übung wird gewährleistet. } \\
\text { (Art. 4; 2) }\end{array}$ \\
\hline $\begin{array}{l}\text { Kein Zwang } \\
\text { zum Kriegs- } \\
\text { dienst mit der } \\
\text { Waffe gegen das } \\
\text { eigene Gewis- } \\
\text { sen/Recht auf } \\
\text { Kriegsdienst- } \\
\text { verweigerung } \\
\end{array}$ & $\begin{array}{l}\text { Die Sozialdemokratische Partei } \\
\text { Deutschlands stellt sich schüt- } \\
\text { zend vor jeden Bürger, der aus } \\
\text { Gewissensgründen den Dienst } \\
\text { mit der Waffe oder an Massen- } \\
\text { vernichtungsmitteln verweigert. } \\
\text { (S. 73) }\end{array}$ & $\begin{array}{l}\text { Niemand darf gegen sein } \\
\text { Gewissen zum Kriegsdienst } \\
\text { mit der Waffe gezwungen } \\
\text { werden. [...] (Art. 4;3) }\end{array}$ \\
\hline $\begin{array}{l}\text { Freiheit der } \\
\text { Meinungsäuße- } \\
\text { rung }\end{array}$ & $\begin{array}{l}\text { Freiheit [...] der Verkündigung } \\
\text { [ist] zu sichern. Eine religiöse } \\
\text { oder weltanschauliche Verkün- } \\
\text { digung darf nicht parteipolitisch } \\
\text { oder zu antidemokratischen } \\
\text { Zwecken mißbraucht werden. } \\
\text { (S. 81) } \\
\text { Alle Vorrechte zu Bildungsein- } \\
\text { richtungen müssen beseitigt } \\
\text { werden. (S.70) }\end{array}$ & $\begin{array}{l}\text { Jeder hat das Recht, seine } \\
\text { Meinung in Wort, Schrift und } \\
\text { Bild frei zu äußern und zu } \\
\text { verbreiten und sich aus allge- } \\
\text { mein zugänglichen Quellen } \\
\text { ungehindert zu unterrichten. } \\
\text { [...] (Art. 5; 1) }\end{array}$ \\
\hline $\begin{array}{l}\text { Meinungsfrei- } \\
\text { heit der Institu- } \\
\text { tionen }\end{array}$ & $\begin{array}{l}\text { Presse, Rundfunk, Fernsehen } \\
\text { und Film erfüllen öffentliche } \\
\text { Aufgaben. Sie müssen in Frei- } \\
\text { heit und Unabhängigkeit überall } \\
\text { und unbehindert Informationen } \\
\text { sammeln, bearbeiten, verbreiten } \\
\text { und unter eigener Verantwor- } \\
\text { tung Meinungen bilden und } \\
\text { aussprechen dürfen. (S. 72) }\end{array}$ & $\begin{array}{l}\text { Die Pressefreiheit und die } \\
\text { Freiheit der Berichterstattung } \\
\text { durch Rundfunk und Film } \\
\text { werden gewährleistet. [...] } \\
\text { (Art. 5; 1) }\end{array}$ \\
\hline
\end{tabular}




\begin{tabular}{|c|c|c|}
\hline $\begin{array}{l}\text { Freiheit von } \\
\text { Lehre, Wissen- } \\
\text { schaft, For- } \\
\text { schung und } \\
\text { Kunst }\end{array}$ & 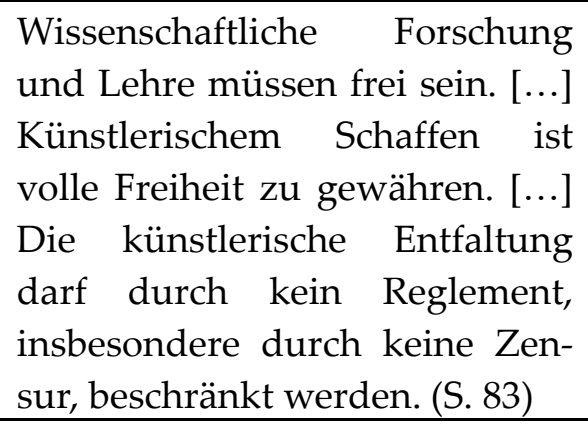 & $\begin{array}{l}\text { Kunst und Wissenschaft, } \\
\text { Forschung und Lehre sind frei. } \\
{[\ldots](\text { Art. } 5 ; 3)} \\
\text { [...] Eine Zensur findet nicht } \\
\text { statt. (Art. } 5 ; 1)\end{array}$ \\
\hline $\begin{array}{l}\text { Schutz von Ehe } \\
\text { und Familie }\end{array}$ & $\begin{array}{l}\text { Staat und Gesellschaft haben die } \\
\text { Familie zu schützen, zu fördern } \\
\text { und zu stärken. (S. 80) }\end{array}$ & $\begin{array}{l}\text { Ehe und Familie stehen unter } \\
\text { dem besonderen Schutze der } \\
\text { staatlichen Ordnung. } \\
(\text { Art. } 6 ; 1)\end{array}$ \\
\hline Familienrechte & $\begin{array}{l}\text { Mütter von vorschulpflichtigen } \\
\text { und schulpflichtigen Kindern } \\
\text { dürfen nicht genötigt sein, aus } \\
\text { wirtschaftlichen Gründen einem } \\
\text { Erwerb nachzugehen. (S. 80) }\end{array}$ & $\begin{array}{l}\text { Jede Mutter hat Anspruch auf } \\
\text { den Schutz und die Fürsorge } \\
\text { der Gemeinschaft. (Art. } 6 ; 4 \text { ) }\end{array}$ \\
\hline $\begin{array}{l}\text { Versammlungs- } \\
\text { freiheit }\end{array}$ & $\begin{array}{l}\text { Er [der Proletarier] erstritt sich } \\
\text { die Versammlungsfreiheit, das } \\
\text { Recht zum gewerkschaftlichen } \\
\text { Zusammenschluß, das Tarifrecht } \\
\text { und das Streikrecht. (S. 85) }\end{array}$ & $\begin{array}{l}\text { Alle Deutschen haben das } \\
\text { Recht, sich ohne Anmeldung } \\
\text { oder Erlaubnis friedlich und } \\
\text { ohne Waffen zu versammeln. } \\
\text { (Art. 8; 1) }\end{array}$ \\
\hline $\begin{array}{l}\text { Vereinigungs- } \\
\text { freiheit }\end{array}$ & $\begin{array}{l}\text { Die Verbände, in denen sich } \\
\text { Menschen der verschiedenen } \\
\text { Gruppen und Schichten zu } \\
\text { gemeinsamen Zwecken zusam- } \\
\text { menschließen, sind notwendige } \\
\text { Einrichtungen der modernen } \\
\text { Gesellschaft. (S. 72) } \\
\text { Alle Arbeiter, Angestellten und } \\
\text { Beamten haben das Recht, sich } \\
\text { in Gewerkschaften zusammen- } \\
\text { zuschließen. [...] Die Gewerk- } \\
\text { schaften kämpfen um einen } \\
\text { gerechten Anteil der Arbeit- } \\
\text { nehmer am Ertrag der gesell- } \\
\text { schaftlichen Arbeit und um das } \\
\text { Recht auf Mitbestimmung im } \\
\text { wirtschaftlichen und sozialen } \\
\text { Leben. (S. 78) }\end{array}$ & $\begin{array}{l}\text { Das Recht, zur Wahrung und } \\
\text { Förderung der Arbeits- und } \\
\text { Wirtschaftsbedingungen Ver- } \\
\text { einigungen zu bilden, ist für } \\
\text { jedermann und für alle Berufe } \\
\text { gewährleistet. [...] (Art. 9; 3) }\end{array}$ \\
\hline $\begin{array}{l}\text { Unverletzlich- } \\
\text { keit/Schutz der } \\
\text { Wohnung }\end{array}$ & $\begin{array}{l}\text { Sie [die Wohnung] ist die Heim- } \\
\text { stätte der Familie. Sie muß } \\
\text { deshalb auch weiterhin sozialen } \\
\text { Schutz genießen [...]. (S. 80) }\end{array}$ & $\begin{array}{l}\text { Die Wohnung ist unverletz- } \\
\text { lich. (Art. 13; 1) }\end{array}$ \\
\hline
\end{tabular}




\begin{tabular}{|c|c|c|}
\hline $\begin{array}{l}\text { Garantie des } \\
\text { Eigen- } \\
\text { tums/Gewährlei } \\
\text { stung von } \\
\text { Erbrecht }\end{array}$ & $\begin{array}{l}\text { Das private Eigentum an Pro- } \\
\text { duktionsmitteln hat Anspruch } \\
\text { auf Schutz und Förderung [...]. } \\
\text { (S. } 75 \text { ) } \\
\text { Das private Eigentum des Bau- } \\
\text { ern am Boden wird bejaht. } \\
\text { (S. } 77)\end{array}$ & $\begin{array}{l}\text { Das Eigentum und das } \\
\text { Erbrecht werden gewährleis- } \\
\text { tet. [...] (Art. 14; 1) }\end{array}$ \\
\hline $\begin{array}{l}\text { Vergesellschaf- } \\
\text { tung }\end{array}$ & $\begin{array}{l}\text { Gemeineigentum ist eine legiti- } \\
\text { me Form der öffentlichen Kon- } \\
\text { trolle, auf die kein moderner } \\
\text { Staat verzichtet. (S. 76) }\end{array}$ & $\begin{array}{l}\text { Grund und Boden, Natur- } \\
\text { schätze und Produktionsmittel } \\
\text { können zum Zwecke der } \\
\text { Vergesellschaftung durch ein } \\
\text { Gesetz [...] in Gemeineigen- } \\
\text { tum oder in andere Formen } \\
\text { der Gemeinwirtschaft über- } \\
\text { führt werden. [...] (Art. 15) }\end{array}$ \\
\hline $\begin{array}{l}\text { Bu } \\
\text { Lär }\end{array}$ & $\begin{array}{l}\text { Durch Verschmelzung des de- } \\
\text { mokratischen mit dem sozialen } \\
\text { und dem Rechtsgedanken soll } \\
\text { der Staat zum Kulturstaat wer- } \\
\text { den [...]. Die Sozialdemokrati- } \\
\text { sche Partei Deutschlands be- } \\
\text { kennt sich zur Demokratie, in } \\
\text { der die Staatsgewalt vom Volke } \\
\text { ausgeht }[\ldots . . . \text { (S. } 71)\end{array}$ & $\begin{array}{l}\text { Die Bundesrepublik Deutsch- } \\
\text { land ist ein demokratischer } \\
\text { und sozialer Bundesstaat. (Art. } \\
\text { 20; 1) } \\
\text { Alle Staatsgewalt geht vom } \\
\text { Volke aus. Sie wird vom Volke } \\
\text { in Wahlen und Abstimmungen } \\
\text { und durch besondere Organe } \\
\text { der Gesetzgebung, der voll- } \\
\text { ziehenden Gewalt und der } \\
\text { Rechtsprechung ausgeübt. } \\
\text { (Art. 20;2) }\end{array}$ \\
\hline $\begin{array}{l}\text { Selbstverwal- } \\
\text { tung und } \\
\text { Selbstverant- } \\
\text { wortung von } \\
\text { Gemeinden }\end{array}$ & $\begin{array}{l}\text { Freie Gemeinden sind unerläß- } \\
\text { lich für eine lebendige Demokra- } \\
\text { tie. Deshalb bekennt sich die } \\
\text { Sozialdemokratische Partei } \\
\text { Deutschlands zu den Grundsät- } \\
\text { zen der Gemeindefreiheit ein- } \\
\text { schließlich der bürgerschaftli- } \\
\text { chen Selbstverwaltung [...]. } \\
\text { (S.72) }\end{array}$ & $\begin{array}{l}\text { Den Gemeinden muß das } \\
\text { Recht gewährleistet sein, alle } \\
\text { Angelegenheiten der örtlichen } \\
\text { Gemeinschaft [...] in eigener } \\
\text { Verantwortung zu regeln. } \\
\text { Auch die Gemeindeverbände } \\
\text { haben im Rahmen ihres ge- } \\
\text { setzlichen Aufgabenbereiches } \\
\text { nach Maßgabe der Gesetze das } \\
\text { Recht der Selbstverwaltung. } \\
\text { (Art. 28;2) }\end{array}$ \\
\hline Rechtsprechung & $\begin{array}{l}\text { Die Richter bedürfen der äuße- } \\
\text { ren und inneren Unabhängig- } \\
\text { keit, um im Namen des Volkes } \\
\text { allein dem Recht zu dienen. } \\
\text { (S. 72) }\end{array}$ & $\begin{array}{l}\text { Die Richter sind unabhängig } \\
\text { und nur dem Gesetze unter- } \\
\text { worfen. (Art. 97; 1) }\end{array}$ \\
\hline
\end{tabular}


Die Gegenüberstellung der korrespondierenden Textstellen macht deutlich, dass sich bestimmte Themenpunkte des Parteiprogramms deutlich an der Wortwahl des Grundgesetztextes orientieren. Das Godesberger Programm legitimiert die eigenen Aussagen und stützt diese dadurch, dass es die im Grundgesetz verankerten Formulierungen zitiert und als eigene Grundwerte betrachtet. Auf den gesetzlich festgelegten und allgemeingültigen Gesetzesformulierungen baut die SPD ihre weitere Programmatik auf. Von der religiös legitimierten Normsetzung über ihre staatliche und gesellschaftliche Regulierungsfunktion bis hin zu individuellen Bedürfnissen und Ansprüchen der Bürger gehören die Grundrechte zu den tragenden und unumstößlichen Grundlagen eines modernen Staates;

ihre Chancen auf Durchsetzung oder Erhaltung ergeben sich nicht nur aus ihrer normativen Qualität, sondern sind abhängig von den jeweiligen politischen Bedingungen. Ihre Festschreibung in Deklarationen und Verfassungen ist ein wichtiger Schritt, ihnen gesellschaftliche Achtung und rechtliche Geltung zu verschaffen. ${ }^{212}$

Geltung und Achtung verschafft die SPD den Thesen dadurch, dass sie diese in ihr Programm aufnimmt; die Referenz der SPD auf das Grundgesetz ist als Achtung und Wertschätzung aufzufassen. Durch die Übernahme der Rechte und Gebote des Grundgesetzes steuert die SPD weiterhin dem möglichen Vorwurf entgegen, ihre Programmatik basiere nicht auf dem Boden der Verfassung der Bundesrepublik Deutschland. Die SPD macht die Gesetzesformulierungen zur Grundlage ihres eigenen Programms und legitimiert dieses dadurch. Diese Feststellung ist allerdings keine allgemeingültige Voraussetzung für die Formulierungen des Godesberger Programms, denn viele Themen werden nicht wörtlich, sondern nur sinngemäß aufgegriffen. Jedoch bleiben viele auf dem Gesetzestext basierende Forderungen unklar. So ist nicht eindeutig nachzuvollziehen, ob die Forderung Künstlerischem Schaffen ist volle Freiheit zu gewähren einerseits bedeutet, dass die Forderung zum Zeitpunkt der Formulierung (im Jahre 1959) nicht erfüllt war und deshalb gefordert wurde, oder ob sie andererseits heißen soll, dass die künstlerische Freiheit auch weiterhin wie bisher zu gewährleisten ist; "das Godesberger Programm sucht in seinen Formulierungen geradezu, so hat man den Eindruck, diese Ambivalenz. ${ }^{\text {} 213}$ In dieser Hinsicht unterscheidet sich die Verwendung solcher Forderungen im Godesberger Programm grundlegend von der des Grundgesetzes „das [...], im Unterschied zu einem Parteiprogramm, indem es einen Sachverhalt als Tatsache beschreibt,

212 Grundrechte. In: Informationen zur politischen Bildung 239. Hg. v. Bundeszentrale für politische Bildung. Bonn 1993, S. 1

213 Hermanns 1989, S. 126 
damit, eo ipso, eine Tatsache - als rechtliche Tatsache - schafft." ${ }^{214}$ Die Äußerungen des Grundgesetztextes Alle Menschen sind vor dem Gesetz gleich (Art. 3; 1) und Männer und Frauen sind gleichberechtigt (Art. 3; 2) sind somit keine Forderungen, sondern Festlegungen, die aufgrund der Erwähnung im Grundgesetztext als rechtliche legitimiert gelten: Alle Menschen in der Gesellschaft sind ebenso gleich vor dem Gesetz wie Männer und Frauen in der Gesellschaft gleichberechtigt sind. Ob letzteres auch de facto so ist, ist in diesem Zusammenhang unerheblich, eine Zuwiderhandlung der Grundgesetzartikel würde dennoch strafrechtlich verfolgt. Es geht hier um die Festlegung durch das Grundgesetz: Anstelle einer Forderung werden Tatsachen geschaffen; die intendierte Textwirkung des Grundgesetztextes ist die „RECHTSGELTUNG in Institutionen und BEFOLGUNG durch Rechtssubjekte." ${ }^{215}$ Dieses Bewirkungsziel wird im Grundgesetztext explizit formuliert: „Die nachfolgenden Grundrechte binden Gesetzgebung, vollziehende Gewalt und Rechtsprechung als unmittelbar geltendes Recht." (Art. 1;3)

Die Forderungen eines Parteiprogramms werden jedoch erst dann zur Festlegung, wenn die Partei Teil einer Regierungskoalition ist oder einen Gesetzentwurf auf Basis einer im Parteiprogramm formulierten Forderung einbringt. Die Formulierung einer These bleibt im Parteiprogramm aber vorerst Beschreibung einer faktischen Tatsache, während die Formulierung im Grundgesetz als Schaffung einer Tatsache betrachtet werden kann. ${ }^{216}$ Das Grundgesetz als Verfassungstext ist daher der Kategorie regulierender Texte zuzuordnen: „Normen sind hier sowohl die SollRegeln, denen alle Bürger verpflichtet werden, als auch die Verfahren und Regeln, nach denen staatliche Institutionen gebildet und nach denen sie wirksam werden. ${ }^{217}$

Jedoch bleibt trotz der formalen und inhaltlichen Übereinstimmungen des Godesberger Programms als Parteiprogramm mit dem Grundgesetz als Verfassungstext ein wesentlicher (stilistischer) Unterschied festzuhalten, der insbesondere bei der späteren Betrachtung der Schlagwörter in den Parteiprogrammen erneut deutlich wird:

214 Hermanns 1989, S. 126

215 v. Polenz 1985, S. 211

216 Allerdings gibt es auch Formulierungen im Grundgesetztext, die darauf hinweisen, dass ein angestrebter Zustand erst noch geschaffen werden muss; die Formulierung an sich reicht zur Schaffung der Tatsache offenbar nicht aus. Diese Feststellung kann durch folgende Forderung gestützt werden: „Den unehelichen Kindern sind durch die Gesetzgebung die gleichen Bedingungen für ihre leibliche und seelische Entwicklung und ihre Stellung in der Gesellschaft zu schaffen wie den ehelichen Kindern." (Art. 6; 5)

217 Strauß 1986a, S. 40 
Die Sprache der Gesetzgebung, Rechtsprechung und Verwaltung ist durch Sachlichkeit und Abstraktheit gekennzeichnet. Die Sprache der Politik ist unter anderem auch Meinungssprache, sie ist argumentativ, sie schließt Emotion und Polemik mit ein. Würden weniger Politiker wie Oberamtsräte sprechen, wäre die viel beklagte Parteienverdrossenheit vielleicht geringer. ${ }^{218}$

An dieser Stelle soll noch der Hinweis auf die syntaktische Erscheinungsform beider Texte hingewiesen. Im Rahmen dieser Untersuchung kann keine kontrastive Stiluntersuchung über die Satzkomplexität beider Texte erfolgen, jedoch scheint der Grundgesetztext überwiegend aus Parataxen zu bestehen, während die Parteiprogramme keinem einheitlichen Satzbaumuster folgen und sich auch parteispezifisch unterscheiden, jedoch häufige hypotaktischen Satzmuster beinhalten. Bereits innerhalb eines speziellen Parteiprogramms unterscheidet sich der Satzbau des Textes von dem der vorangesellten Präambel teilweise grundlegend.

$\mathrm{Da}$ in der Abhandlung dieses und der vorangegangenen Kapitel, wie auch im oben zitierten Textauszug über die Grundrechte der Ausdruck Grundwerte gefallen ist, soll an dieser Stelle um der Vollständigkeit willen erwähnt werden, dass die SPD oder allgemein die Parteien diesen Ausdruck nicht mit Themen oder anderen Instanzen innerhalb des jeweiligen Textes verwechselt oder verallgemeinert sehen wollen. Ein bestimmtes Grundrecht ist nicht zwangsläufig auch als Grundwert zu verstehen:

Die Kommission wollte präzise unterschieden wissen zwischen Grundrechten [...], Ordnungsprinzipien [...], wertbezogenen Institutionen $[\ldots]$, Rechtsgütern [...], Tugenden [...] und den Grundwerten politischer Parteien. Wo Sozialdemokraten von Grundwerten sprechen, meinen sie das, was sie früher Prinzipien nannten, also die drei Leitgedanken, Leitbegriffe der Französischen Revolution: Freiheit, Gleichheit und Brüderlichkeit, die als Freiheit, Gerechtigkeit und Solidarität in das Godesberger Programm eingingen und seither auch in den Programmen anderer Parteien Aufnahme fanden. ${ }^{219}$

Hinsichtlich der in der zitierten Textpassage formulierten Begriffsdifferenzierungen beinhalten die Grundrechte beispielsweise die Meinungsfreiheit; mit Ordnungsprinzipien sind Rechtsstaatlichkeit, Sozialstaatlichkeit oder Gewaltenteilung gemeint; wertbezogene Institutionen sind die Ehe, Familie, Gemeinden oder der Staat; Gesundheit, Leben oder Freizügigkeit werden als Rechtsgüter verstanden; Tugenden des politischen Bürgers sind beispielsweise Zivilcourage, Offenheit, Toleranz oder Recht-

218 Geißler, Heiner: „Sprache und Politik. Kampf um Begriffe.“ In: Germanistik Forschungsstand und Perspektiven. Vorträge des Deutschen Germanistentages 1984 (Teil 1). Hg. v. Georg Stötzel. Berlin/New York 1985, S. 229 
lichkeit; als Grundwerte der konkurrierenden politischen Parteien werden Freiheit, Frieden, Gerechtigkeit oder Solidarität angesehen. Dabei laufen insbesondere die Grundwerte Gefahr, als abstrakte Allgemeinplätze an Bedeutung zu verlieren:

Grundwerte, zumal wenn sie allen Parteien gemeinsam sind, stehen in der Gefahr, abstrakt zu bleiben und über dem harten Geschäft des politischen Alltags außer Sichtweite zu geraten.220

Die beiden oben zitierten Aussagen von EPPLER (1984) können verifiziert werden: Insbesondere die Grundwerte Freiheit, Gerechtigkeit und Solidarität als die "drei Leitgedanken“ werden parteiübergreifend in nahezu allen Grundsatzprogrammen aufgegriffen (die FDP geht dabei explizit auf die Ereignisse der Französischen Revolution von 1775 und 1789 ein). Es handelt sich daher um Topoi (Freiheitstopos, Gerechtigkeitstopos), die von den Parteien aufgegriffen werden (müssen). Sie bleiben jedoch häufig in abstrakter Verwendung bestehen und werden nicht konkretisiert:

(33) (a) Freiheit, Gerechtigkeit und Solidarität, die aus der gemeinsamen Verbundenheit folgende gegenseitige Verpflichtung, sind die Grundwerte des sozialistischen Wollens. (Godesberger Programm 1959, S. 69)

(b) Es ist das Zeitalter des Friedens und der sozialen Sicherheit, der Menschenwürde und Brüderlichkeit, der Freiheit und Gerechtigkeit, der Menschlichkeit und Lebensfreude. (SED 1963, S. 208)

(c)Die Völker der Welt aber sehnen sich nach Frieden und Freiheit, nach Gerechtigkeit und Selbstbestimmung, Sicherheit nach innen und außen, nationaler Unabhängigkeit, sozialem Wohlstand und einem menschenwürdigen Dasein. (NPD 1973, S. 342)

(d) Die CDU vereint als moderne Volkspartei Männer und Frauen aller Schichten in dem Willen, das deutsche Volk in Frieden, Freiheit und Gerechtigkeit zu einen. (CDU 1971, S. 47)

(e) Grundlage und Orientierung unseres politischen Handelns sind das christliche Verständnis vom Menschen und die daraus abgeleiteten Grundwerte Freiheit, Solidarität und Gerechtigkeit. Die Grundwerte erfordern und begrenzen sich gegenseitig. Keiner erfüllt ohne die anderen seinen Sinn. Ihre Gewichtung untereinander richtig $\mathrm{zu}$ gestalten ist Kern der politischen Auseinandersetzung. Die Grundwerte sind als unteilbare Menschenrechte nicht auf nationale Grenzen beschränkt und sind verpflichtende Grundlage für unsere Außenpolitik. (CDU 1994, S. 369)

(f) Sie zielt auf eine in der Sache nicht weniger als 1775 und 1789 revolutionäre, im wörtlichen Sinne umwälzende, in den westli- 
chen Industriestaaten und Massendemokratien nun endlich auf evolutionärem Wege durchsetzbare Demokratisierung der Gesellschaft, aus demselben Gedanken der ,Freiheit, Gleichheit und Brüderlichkeit!', aus dem auch die Demokratisierung des Staates ihren Ursprung nahm. Diese neue Phase der Demokratisierung und Liberalisierung, im ursprünglichen und nicht dem heute oft mißbrauchten Sinne dieser Worte, entspringt aus einem gewandelten Verständnis der Freiheit, das dem modernen Liberalismus die neue politische Dimension eines nicht mehr nur Demokratischen, sondern zugleich Sozialen Liberalismus erschließt. (FDP 1971, S. 250)

(g) $\quad$ Freiheit Gerechtigkeit und Solidarität sind die Grundwerte des Demokratischen Sozialismus. [...] Freiheit, Gerechtigkeit und Solidarität bedingen einander und stützen sich gegenseitig. Gleich im Rang, einander erläuternd, ergänzend und begrenzend erfüllen sie ihren Sinn. (SPD 1989, S. 12/13)

(h) Sozialismus ist für uns ein Wertesystem, in dem Freiheit, Gleichheit und Solidarität, Emanzipation, Gerechtigkeit, Erhalt der Natur und Frieden untrennbar miteinander verbunden sind. [...] Freiheit, Gleichheit und Solidarität bilden den Inhalt von Gerechtigkeit. (PDS 2003, S. 2/3)

(i) Wir wollen eine ökologisch nachhaltige, freiheitliche demokratische und solidarische Welt gestalten, ohne Hunger, Armut und Krieg. (B 90/GRÜNE 2002, S. 17)

Zwar erhebt die SPD in Äußerung (a) den Anspruch, die Grundwerte als Grundwerte des Sozialismus zu etablieren, es handelt sich bei der traditionsreichen Trias Freiheit, Gerechtigkeit und Solidarität jedoch um Werte, die in umgekehrter Reihenfolge bereits durch den Text des Deutschlandlieds von August Heinrich Hoffmann von FALLERSLEBEN aus dem Jahre 1841 hinlänglich bekannt sind. Danach entspricht der Einigkeit die Solidarität, dem Recht entspricht die Gerechtigkeit und der Grundwert Freiheit bleibt bestehen. Beide Formeln können als Paraphrase der Parole der Französischen Revolution von 1789 mit ihrem Ruf nach Liberté, Egalité, Fraternité betrachtet werden. In Äußerung (f) wird die Französische Revolution als geschichtliches Ereignis explizit genannt und positiv bewertet; die damaligen Zielbestrebungen werden als gegenwärtig gültig und damit zu den Basiswerten erklärt, auf die sich der Soziale Liberalismus stützt. Bei Betrachtung der Äußerungen (b) und (c) fällt eine inhaltliche bzw. thematische Dichte auf, indem neben der expliziten Berufung auf die Menschenwürde und den Grundwerten auch Tugenden (Menschlichkeit), Ordnungsprinzipien (soziale Sicherheit, Sicherheit nach innen und außen) und die Grundrechte (Selbstbestimmung) innerhalb eines Satzes abgehandelt werden. Hinsichtlich der Äußerung (d) sei angemerkt, dass die SPD den Grundwerten in ihrem Grundsatzprogramm von 1989 einen hohen Stellenwert einräumt. So gibt es in dem eigenständigen Kapitel Grundwerte des Demokratischen Sozialismus die drei Themenbereiche Frei- 
heit, Gerechtigkeit und Solidarität; diese drei Begriffe sind als einzige sowohl unterstrichen als auch kursiv gesetzt. Die im Programm benannten Grundwerte haben eine dreifache Funktion:

Sie sollen erstens ,Kriterien' abgeben für die Analyse der gesellschaftlichen Wirklichkeit, zweitens ,Maßstab' einer neuen und besseren Gesellschaftsordnung sein und schließlich drittens als Handlungs,Orientierung' für die einzelnen SPD-Mitglieder gelten. ${ }^{221}$

Auch die anderen Parteien scheinen die Grundwerte in ihren Parteiprogrammen nach diesem Verständnis zu verwenden. Hierbei soll allerdings das Grundsatzprogramm der GRÜNEN erwähnt werden, dass bei der Formulierung von den anderen Programmen abweicht (i). Zwar werden implizit die Grundwerte gefordert, die Ausdrücke Solidarität, Freiheit und Gerechtigkeit werden hier jedoch nicht explizit in Form einer Trias genannt; eine Begründung könnten die verschiedenen Nebenbedeutungen sein, die diesen Abstrakta eingeräumt werden: „Den Begriff der Freiheit überlassen wir nicht jenen, die ihn mit Vorliebe verengen auf reine Marktfreiheit, die Freiheit des Ellenbogens" (B 90/GRÜNE 2003, S. 11). Die Ausdrücke werden zwar an anderer Stelle des Programms verwendet, sie sind jedoch dann immer an einen konkreten Kontext gebunden. Der Begriff Gerechtigkeit wird in Äußerung (i) zwar nicht verwendet, sinngemäß ergibt er sich aber aus der Formulierung.

\subsection{Die Präambeln im Godesberger Programm und im Grundgesetz}

Auffällig ist, dass das Godesberger Programm nicht nur inhaltlich, sondern auch formal an den Text des Grundgesetztes angelehnt ist. Bereits die Tatsache, dass eine Präambel existiert zeigt die Anlehnung an den Grundgesetztext auf. Beide Texte ähneln sich im Aufbau hinsichtlich der Formulierung einer Präambel; so findet sich im Godesberger Programm eine Präambel,

die an Feierlichkeit der Präambel des Grundgesetzes nicht nachsteht [...] und die, wie die Präambel des Grundgesetzes, graphisch als Lyrik in freien Rhythmen erscheint (im Grundgesetz noch eher zu deuten als überkommener Formularstil mit graphischen Hervorhebungen zum Zwecke der leichteren Lesbarkeit; aber auch da ist die archaisierende Feierlichkeit nicht zu verkennen). ${ }^{222}$

Die nachfolgend wiedergegebene Präambel unterscheidet sich somit in verschiedener Hinsicht grundlegend von den anderen Kapiteln des Godesberger Programms. Die Präambel wird an dieser Stelle mit der Präambel des Grundgesetztextes verglichen und später einer detaillierteren

\footnotetext{
221 IMSF 1987, S. 43

222 Hermanns 1989, S. 127
} 
Analyse unterzogen. Der zitierte Text gibt die Präambel mit den graphischen Hervorhebungen, den Absätzen und der Fettschrift originalgetreu wieder:

1. Das ist der Wider s pru ch unserer Zeit,

2. daß der Mensch die Urkraft des Atoms entfesselte und

3. sich jetzt vor den Folgen fürchtet;

4. daß der Mensch die Produktivkräfte aufs höchste entwickelte,

5. ungeheure Reichtümer ansammelte, ohne allen einen

6. gerechten Anteil an dieser gemeinsamen Leistung zu verschaffen;

7. daß der Mensch sich die Räume dieser Erde unterwarf,

8. die Kontinente zueinander rückte, nun aber

9. in Waffen starrende Machtblöcke die Völker mehr

10. voneinander trennen als je zuvor und totalitäre Systeme

11. seine Freiheit bedrohen.

12. Darum fürchtet der Mensch, gewarnt durch die Zerstörungskriege

13. und Barbareien seiner jüngsten Vergangenheit, die eigene Zukunft,

14. weil in jedem Augenblick an jedem Punkt der Welt

15. durch menschliches Versagen das Chaos der Selbstvernichtung

16. ausgelöst werden kann.

17. Aber da ist auch die $\mathrm{H}$ of $\mathrm{f} \mathbf{n} \mathrm{u} \mathrm{n}$ dieser Zeit,

18. daß der Mensch im atomaren Zeitalter sein Leben erleichtern,

19. von Sorgen befreien und Wohlstand für alle schaffen kann,

20. wenn er seine täglich wachsende Macht über die Naturkräfte

21. nur für friedliche Zwecke einsetzt;

22. daß der Mensch den Weltfrieden sichern kann, wenn er

23. die internationale Rechtsordnung stärkt,

24. das Mißtrauen zwischen den Völkern mindert

25. und das Wettrüsten verhindert;

26. daß der Mensch dann zum erstenmal in seiner Geschichte

27. jedem die Entfaltung seiner Persönlichkeit in einer gesicherten

28. Demokratie ermöglichen kann zu einem Leben in kultureller

29. Vielfalt, jenseits von Not und Furcht.

30. Diesen Widerspruch aufzulösen, sind wir Me $\mathrm{n} \mathbf{s} \mathrm{ch}$ e $\mathrm{n}$

31. aufgerufen. In unsere Hand ist die Verantwortung gelegt für eine

32. glückliche Zukunft oder für die Selbstzerstörung der Menschheit. 


\section{Nur durch eine neue und bessere Ordnung der Gesellschaft \\ 34. öffnet der Mensch den Weg in seine Freiheit.}

\section{Diese neue und bessere Ordnung erstrebt \\ 36. der demokratische Sozialismus. ${ }^{223}$}

Die These des oben angeführten Zitats soll durch die folgende Gegenüberstellung signifikanter Textstellen der Präambeln des Godesberger Programms und des Grundgesetztextes bekräftigt werden:

\begin{tabular}{|c|c|c|}
\hline Thema & $\begin{array}{c}\text { Präambel des Godes- } \\
\text { berger Programms }\end{array}$ & Präambel des Grundgesetztextes \\
\hline & $\begin{array}{l}\text { Das ist der Widerspruch } \\
\text { unserer Zeit, daß der } \\
\text { Mensch [...]. } \\
\text { Diesen Widerspruch } \\
\text { aufzulösen, sind wir } \\
\text { Menschen aufgerufen. } \\
\text { In unsere Hand ist die } \\
\text { Verantwortung gelegt für } \\
\text { eine glückliche Zukunft } \\
\text { oder für die Selbstzerstö- } \\
\text { rung der Menschheit. [...] } \\
\text { Diese neue und bessere } \\
\text { Ordnung erstrebt der } \\
\text { demokratische Sozialis- } \\
\text { mus. (S. 68/69) }\end{array}$ & $\begin{array}{l}\text { Im Bewußtsein seiner Verantwortung vor } \\
\text { Gott und den Menschen, von dem Willen } \\
\text { beseelt, seine nationale und staatliche Einheit } \\
\text { zu wahren und als gleichberechtigtes Glied in } \\
\text { einem vereinten Europa dem Frieden der } \\
\text { Welt zu dienen, hat das Deutsche Volk [...] } \\
\text { um dem staatlichen Leben für eine Über- } \\
\text { gangszeit eine neue Ordnung zu geben, kraft } \\
\text { seiner verfassungsgebenden Gewalt dieses } \\
\text { Grundgesetz der Bundesrepublik Deutsch- } \\
\text { land beschlossen. Es hat auch für jene Deut- } \\
\text { schen gehandelt, denen mitzuwirken versagt } \\
\text { war. }\end{array}$ \\
\hline $\begin{array}{l}\text { Freiheit und } \\
\text { Selbstbestim- } \\
\text { mung des Men- } \\
\text { schen/ } \\
\text { Wiedervereini- } \\
\text { gung Deutsch- } \\
\text { lands }\end{array}$ & $\begin{array}{l}\text { In seinem Sinne erstrebt } \\
\text { sie die Einheit Deutsch- } \\
\text { lands in gesicherter Frei- } \\
\text { heit. [...] } \\
\text { Erst in einem wiederver- } \\
\text { einigten Deutschland } \\
\text { wird das ganze Volk in } \\
\text { freier Selbstbestimmung } \\
\text { Inhalt und Form von Staat } \\
\text { und Gesellschaft gestalten } \\
\text { können. (S. 71) }\end{array}$ & $\begin{array}{l}\text { Das gesamte Deutsche Volk bleibt aufgefor- } \\
\text { dert, in freier Selbstbestimmung die Einheit } \\
\text { und Freiheit Deutschlands zu vollenden.224 } \\
\text { Im Bewußtsein seiner Verantwortung vor } \\
\text { Gott und den Menschen, von dem Willen } \\
\text { beseelt, als gleichberechtigtes Glied in einem } \\
\text { vereinten Europa dem Frieden der Welt zu } \\
\text { dienen, hat sich das Deutsche Volk kraft } \\
\text { seiner verfassungsgebenden Gewalt dieses } \\
\text { Grundgesetz gegeben. Die Deutschen in den } \\
\text { Ländern [...] haben in freier Selbstbestim- } \\
\text { mung die Einheit und Freiheit Deutschlands } \\
\text { vollendet. [...] (Die Präambel nach der Neu- } \\
\text { fassung durch den Einigungsvertrag vom } \\
\text { 31.08.1990; S. 163) }\end{array}$ \\
\hline
\end{tabular}

223 Godesberger Programm 1959, S. 68/69 
Die gegenübergestellten zitierten Textpassagen bilden nicht den vollständigen Text der jeweiligen Präambel $a b$, sondern stellen lediglich Auszüge daraus dar. Die Präambel des Godesberger Programms umfasst insgesamt 255 Wörter, die des Grundgesetzes nur 79 Wörter; die Präambel des Godesberger Programms ist folglich ausführlicher und umfasst mehr Themengebiete, wie beispielsweise die atomare Bedrohung, (Un-)Gerechtigkeit, Wiedervereinigung, Freiheit, Krieg, Wohlstand, Frieden, Rechtsordnung, Aufrüstung, Entfaltung der Persönlichkeit, Selbstverantwortung und Gesellschaftsordnung, während die Präambel des Grundgesetzes dagegen kompakter und inhaltlich dichter formuliert ist. Weiterhin lässt sich trotz aller Übereinstimmungen ein wesentlicher Unterschied zwischen beiden Präambeln nachweisen: Während die SPD sich in der Präambel des Godesberger Programms explizit als Text-Autor bekennt (Beschlossen vom Außerordentlichen Parteitag der Sozialdemokratischen Partei Deutschlands in Bad Godesberg vom 13. bis 15. November 1959) wird im Grundgesetztext kein konkreter Textverfasser genannt; obwohl es Textautoren gibt (Parlamentarischer Rat) treten diese hinter ihr Produkt zurück. Der Text-Autor wird in der Präambel des Grundgesetzes verschleiert und durch die Formulierung das Deutsche Volk hat beschlossen wird das Volk als "abstrakter kollektiver Verantwortungsträger“225 hingestellt. Hingewiesen werden soll hierbei allerdings auf die veränderte Formulierung der Präambel nach der Neufassung durch den Einigungsvertrag vom 31.08.1990, nach der das Deutsche Volk sich ein Grundgesetz gegeben hat; das Volk ist in dieser Formulierung Text-Autor und TextAdressat zugleich, die Adressaten werden im Grundgesetz jedoch nicht direkt angesprochen.

Es lassen sich aber inhaltliche Entsprechungen nachweisen. So wird das Thema „Frieden der Welt" des Grundgesetzes im Godesberger Programm mit der Formulierung daß der Mensch den Weltfrieden sichern kann behandelt. Diesbezüglich sei noch angemerkt, dass das Godesberger Programm anstelle des syntaktischen Wortgefüges des Grundgesetzes den Ausdruck in Form eines Nominalkompositums verwendet. Das Thema „vereintes Europa" des Grundgesetzes erschließt sich dem Rezipienten des Godesberger Programms nur indirekt durch die Formulierung nun aber in Waffen starrende Machtblöcke die Völker mehr voneinander trennen als je zuvor und totalitäre Systeme seine Freiheit bedrohen. Dabei ist es notwendig zu wissen, dass der Aufbau der Präambel des Godesberger Programms auf

${ }^{224}$ Verfassung des Landes Hessen und Grundgesetz für die Bundesrepublik Deutschland. Mit einer Einführung und einem Anhang: Allgemeine Erklärung der Menschenrechte - Konvention zum Schutze der Menschenrechte und Grundfreiheiten (Auszug) - Charta der Vereinten Nationen. Hg. v. Georg August Zinn und Erwin Stein. Bad Homburg vor der Höhe ${ }^{42} 1989$, S. 171

v. Polenz 1985, S. 35 
zwei sich entsprechenden und gegensätzlichen Teilen beruht; der erste negative Teil wird eingeleitet durch den Satz das ist der Widerspruch unserer Zeit, an den sich nachfolgende Äußerungen anheften. Die Wörter Widerspruch und Hoffnung sind im Parteiprogramm gesperrt gesetzt, um den emphatischen Grundton der Präambel und den Gegensatz nicht nur inhaltlich sondern auch graphisch zu verdeutlichen:

\begin{abstract}
Bei der Emphase erfolgt die Merkmalshervorhebung ohne zusätzliche sprachliche Mittel (implizit) durch eine - im Kontext signalisierte bedeutungsvolle Hervorhebung des Wortes, das als Tropus fungiert, sei es durch Akzent (Schwerton) in der gesprochenen, durch graphische Kennzeichnung (Anführungszeichen, Bindestrich, Fett- oder Sperrdruck) in der geschriebenen Sprache oder durch nachdrückliche Wiederholung. ${ }^{226}$
\end{abstract}

Der Leser interpretiert den Zustand, dass die Völker - obwohl der Mensch die Kontinente zueinander rückte - durch in Waffen starrende Machtblöcke getrennt sind, als einen schlechten Zustand, den es zu beseitigen gilt; die Forderung nach einer vereinigten bzw. vereinten Staatengemeinschaft erschließt sich nur implizit und mag nicht für jeden Rezipienten gleichermaßen nachvollziehbar sein. Der zweite positive Teil der Präambel, der durch den Satz aber das ist auch die Hoffnung dieser Zeit eingeleitet wird verstärkt diesen Eindruck und spricht für die dargestellte Interpretation. Der Bezug auf die Selbstverantwortung und die Souveränität des deutschen Volkes wird ebenfalls in beiden Texten thematisiert.

Hinsichtlich des Aufbaus ist noch zu erwähnen, dass der Grundgesetztext das Kapitel „Die Grundrechte“ direkt an die Präambel anschließt; im Godesberger Programm folgen die Kapitel Grundwerte des Sozialismus und Grundforderungen für eine menschenwürdige Gesellschaft der Präambel unmittelbar. Somit erinnert der formale Aufbau des Godesberger Programms auch in diesem Punkt an den Grundgesetztext.

Die oben genannten möglichen Begründungen für den deutlichen Bezug des Godesberger Programms zu den Aussagen des Grundgesetzes können um den Verweis auf die Geschichte der Bundesrepublik Deutschland erweitert werden. Die Freiheit des einzelnen kann nicht als ungefährdetes Gut betrachtet werden; der Bezug zum Grundgesetz kann somit als Möglichkeit betrachtet werden, den damaligen/heutigen Freiheitsbedrohungen wirksam zu begegnen, da die einzelnen Grundrechte der Verfassung und des Grundgesetzes der Freiheit des einzelnen dienen.

STRABNER (1987) sieht in der Verwendung von Präambeln als politische Symbolik eine besondere Form der Legitimation von Herrschaft ver-

226 Fleischer, Wolfgang/Michel, Georg/Starke, Günter: Stilistik der deutschen Gegenwartssprache. Frankfurt/Main 21996, S. 251 
wirklicht. Dabei ist eine Präambel insbesondere durch die Funktion determiniert und nicht durch den Wahrheitsanspruch oder Wahrheitsgehalt; der Sinn in der Verwendung von Präambeln ist daher,

Handlungen zu bewerten und zu Handlungen anzuspornen, nicht zu informieren. Sie vermitteln nicht Wissen, sondern befriedigen ein anderes Bedürfnis, den Wunsch, nicht auf der Basis materieller oder intellektueller Macht, sondern auf der Grundlage eines moralischen Prinzips regiert zu werden. 227

Bei Betrachtung der oben zitierten Präambel des Godesberger Programms wird an einigen Textstellen deutlich, inwiefern sich der Textabschnitt vom Rest des Parteiprogramms unterscheidet. Die Legitimation erfolgt durch den Appell an die Moral des Rezipienten, der wiederum sprachlich realisiert wird durch den Gebrauch metaphorischer Wendungen (der Mensch entfesselt die Urkraft des Atoms, Zeile 2), durch das Stilmittel der Personifizierung (Machtblöcke starren in Waffen, Zeile 9), durch das Ansprechen des Gemeinschaftsgefühls (unsere Zeit, Zeile 1; alle, Zeile 5/19; gemeinsame Leistung, Zeile 6; jedem, Zeile 27; wir Menschen, Zeile 30; in unsere Hand, Zeile 31) und durch besonders ausdrucksintensive Wörter, die jeweils gegensätzliche Positionen vertreten, wie Chaos $\leftrightarrow$ Ordnung, Weltfrieden $\leftrightarrow$ Zerstörungskriege, Widerspruch $\leftrightarrow$ Hoffnung, Sorgen $\leftrightarrow$ Wohlstand, Vergangenheit $\leftrightarrow$ Zukunft, Verantwortung $\leftrightarrow$ Selbstvernichtung/Selbstzerstörung sowie weiterhin die Ausdrücke Barbareien oder Freiheit.

Somit geht von einer Präambel eine emotive Wirkung auf den Rezipienten aus; formal dient die Präambel als Einleitung bzw. als besondere Form einer Exposition, da die jeweilige Partei in diesem einleitenden Abschnitt ihren Standpunkt deutlich macht, ohne auf konkrete Sachverhalte oder Konzepte einzugehen (dies geschieht in den einzelnen Kapiteln des nachfolgenden Programmtextes). Präambeln finden sich in beiden Parteiprogrammen der GRÜNEN (1980 und 2002) sowie in den Programmen der PDS 2003, der SPD 1989 und der CDU 1971. Die SED stellt ihrem Parteiprogramm von 1963 die Einleitung Ein neuen Zeitalter hat begonnen voraus und bezeichnet diesen Textabschnitt auch als Einleitung, nicht als Präambel (ebenso die FDP 1971). Die Formulierung hat begonnen der SED macht allerdings deutlich, dass die Ideale des Sozialismus nicht für noch uneingelöste Zielvorstellungen, sondern für realisierte, zumindest aber für bereits eingeleitete Grundlagen stehen. Diese Aussage steht somit im Gegensatz zum erstrebten demokratischen Sozialismus des Godesberger Programms. 
In allen untersuchten Parteiprogrammen ist jedoch ein einleitender Textabschnitt vorangestellt, in dem die politische Ausgangslage auf eindringliche Weise skizziert wird, teilweise mit polemischen Nuancen.

\subsubsection{Das Parteiprogramm als Bekenntnisschrift}

Geltung und Achtung verschaffen sich die Parteien jedoch nicht nur dadurch, dass sie sich in ihren Programmen auf das Grundgesetz berufen und diese sich formal oder inhaltlich an Verfassungstexten orientieren; die Parteien scheinen sich auch durch die Berufung auf die Religion durch die Verwendung religiöser Ausdrücke zu legitimieren. Die Referenz der Parteien auf die Religion ist ebenso wie die auf einen Verfassungstext als Achtung und Wertschätzung aufzufassen. Diese Auffassung ist hinsichtlich der Parteien wie der SED, der KPD, der DKP oder der PDS zu bezweifeln, insbesondere hinsichtlich der MARXSCHEN These Sie [die Religion] ist das Opium des Volkes.

Durch die Verwendung religiösen Vokabulars wird ein Parteiprogramm zu einer feierlichen Bekenntnisschrift; es wird dadurch weiterhin dem möglichen Vorwurf entgegengewirkt, die Programmatik basiere nicht auf dem Glauben bzw. die Zielbestrebungen seien mit religiösen, moralischen oder ethischen Einstellungen unvereinbar. In diesem Sinne ist auch die Aufnahme des Ausdrucks christlich im Parteinamen der CDU und CSU zu verstehen.

Zur Feierlichkeit als stilistisches Merkmal trägt die Verwendung des Verbs (sich) bekennen und der Substantive Hoffnung und Bekenntnis bei; diese Ausdrücke können parteiübergreifend in nahezu allen Programmen nachgewiesen werden (lediglich die FDP, die SED, die KPD und die PDS verzichten in ihren Programmen auf die Verwendung von bekennen oder Bekenntnis):

(34) (a) Die Sozialdemokratische Partei Deutschlands bekennt sich zur Verteidigung der freiheitlich-demokratischen Grundordnung. (Godesberger Programm 1959, S. 72/73)

(b) Wir bekennen uns zur parlamentarischen Demokratie. (SPD 1989, S. 50)

(c) Unser Bekenntnis zu Pluralismus der Lebensstile und zur Verschiedenheit der Menschen schließt die Forderung nach Freiheit in den Rechten für alle ein. (B 90/GRÜNE 2002, S. 121)

(d) Sie [die CDU] bekennt sich zum Selbstbestimmungsrecht des ganzen deutschen Volkes, zu einem politisch geeinten Europa und einer Völkergemeinschaft, die den Frieden in der Welt sichern hilft und dem Wohle und der Entwicklung aller Völker dient. (CDU 1971, S. 47) 
(e) Wir Nationaldemokraten bekennen uns zur Vielfalt des Lebens und seiner Erscheinungen in Natur und Geschichte. (NPD 1973, S. 348)

(f) Die CSU bekennt $\underline{\text { sich }}$ zum demokratischen Rechtsstaat. (CSU 1968, S. 234)

(g) Die DKP bekennt sich zu den Prinzipien des proletarischen Internationalismus. (DKP 1969, S. 341)

(h) Aber da ist auch die Hoffnung dieser Zeit, [...]. Darum ist die Hoffnung der Welt eine Ordnung, die auf den Grundwerten des demokratischen Sozialismus aufbaut, [...]. (Godesberger Programm 1959, S. 68/86)

(i) Als Vortrupp der Menschheit war und ist die Sowjetunion stets die große moralische und politische Hoffnung der revolutionären internationalen Arbeiterbewegung und der Völker in ihrem weltweiten Kampf gegen Imperialismus und Krieg. (SED 1963, S. 318)

(j) Mit dieser Veränderung verbinden wir viele Hoffnungen. (B 90/GRÜNE 2002, S. 143)

(k) Im Ersten Weltkrieg enttäuschte die sozialdemokratische Arbeiterbewegung Europas viele in der Hoffnung, sie könne den Frieden erzwingen. [...] Hoffnung entsteht nicht aus der Verdrängung von Gefährdungen, sondern aus Aufklärung im öffentlichen Dialog. Reformpolitik setzt auf Hoffnung. (SPD 1989, S. 8/53)

Einen erwähnenswerten formalen Bezug zu einer konfessionellen Schrift lässt sich hinsichtlich des Programms der SED vermuten, da die Fragestellung „Sozialismus, was ist das?“228 sowie die korrespondierende Form „Sozialismus, das ist:“229 insgesamt 6-mal als Einleitung von sechs unmittelbar aufeinander folgenden Absätzen in der formalen Gestalt der Zehn Gebote wiedergegeben ist. Die Konzeption gleicht in dieser Hinsicht dem 1529 zuerst erschienenen Kleinen Katechismus von Martin Luther (1483-1546), dessen Auslegungen der einzelnen Gebote des Dekalogs stets mit der signifikanten Fragestellung „Was ist das?“230 beginnen. Diese Feststellung kann jedoch keinesfalls als inhaltliche Übereinstimmung, sondern lediglich als analytische Ähnlichkeit bzw. als formale Analogie verstanden werden. Die auf diesem Textvergleich basierende These ist somit gewagt und problematisch zugleich, da die SED auf den Gebrauch

228 SED 1963, S. 211 
der Ausdrücke Bekenntnis oder bekennen in ihrem Programm verzichtet und die DDR bis 1989/90 als kommunistische Diktatur die Kirche und Religionsausübung nicht als wesentlichen Punkt ihrer Programmatik betrachtete: „Weil sozialistische Politik in der Vergangenheit in konfrontativem Denken befangen war, hat sie die Notwendigkeit des Dialogs mit den Religionsgemeinschaften, unter anderem auch mit internationalen Bewegungen religiöser Sozialistinnen und Sozialisten, unterschätzt" (PDS 2003, S. 29). Zwar wird die Religionsausübung in Äußerung (a) explizit gewährleistet, in Äußerung (b) wird sowohl der evangelischen als auch katholischen Konfession eine negative Nebenbedeutung beigemessen:

(35) (a) Sie wahrt den Grundsatz der Trennung von Staat und Kirche. Sie gewährleistet die Religionsausübung im Rahmen der Kirchen und Religionsgemeinschaften. (SED 1963, S. 294)

(b) Die deutschen Imperialisten greifen gleichzeitig mit Unterstützung des reaktionären Klerus, besonders der katholischen Kirche, aber auch einer Reihe evangelischer Kirchenführer, zum Mißbrauch der Religion, um, als Vorkämpfer für das Christentum und das "Abendland" getarnt, ihre aggressiven Revancheziele zu verfolgen. (SED 1963, S. 245)

\subsubsection{Zur Rezeption von Parteiprogrammen}

Parteiprogramme werden primär durch Kommissionen von Fachleuten formuliert, die wissen, daß es vor allem Fachleute in den eigenen Reihen, beim politischen Gegner, in Interessenverbänden und Massenmedien sind, die die Texte lesen, und zwar im Modus des Auswertens. Auswerten bedeutet vor allem, die intertextuelle Potenz des Programms zu nutzen: als Basis für Gesetzesinitiativen, als Steinbruch für Werbespots, als Bezugstext für massenmediale Kommentierung, als Stichwort-Lieferant für polemische Kampagnen etc. ${ }^{231}$

Die Berücksichtigung aller potentieller Wählerschichten und die mögliche Rezeption der Inhalte eines Parteiprogramms haben unmittelbare Auswirkungen auf die Konzeption des Programms und wirken sich auch auf die bereits dargestellten Konversationsmaximen nach GRICE (1979) aus, denn

darüber, was fundiert, was relevant, was informativ und verständlich ist, existieren durchaus unterschiedliche Vorstellungen bei Experten und Laien, Betroffenen und Nicht-Betroffenen, Nahestehenden und Gegnern, Interessierten und Uninteressierten, bei Menschen mit un-

231 Klein 1996a, S. 202 
terschiedlicher ideologischer Orientierung oder mit unterschiedlicher sozialer Gruppenzugehörigkeit. ${ }^{232}$

Bei der Formulierung der Inhalte spielen daher außersprachliche Motive wie Überlegungen zur Adressatengruppe eine zentrale Rolle. Dies verdeutlicht, dass ein komplexes Kommunikationsgeflecht besteht zwischen den Botschaften der Abgeordneten bzw. der Parteien und den Massenmedien einerseits sowie der Partei- und Wählerbasis andererseits als Rezipienten. Dabei gehen die Parteien von einem sowohl emotional als auch rational handelnden Wähler aus. Unterschiedliche Konzeptionen von Parteiprogrammen werden vermutlich entweder den ersteren oder den letzteren Wählertyp erreichen. Insbesondere der rationale Wähler

bedarf klarer politischer Angebote der einzelnen Parteien; er benötigt die weitgehende Transparenz dieses Angebots, nicht die durch unverbindliche Slogans drapierte Aufforderung, sich getrost anzuvertrauen. Dieses Angebot sollte dem Wähler detailliert und so präzis wie möglich gemacht werden. ${ }^{233}$

Auf der Basis dieser Angebote kann der rationale Wähler die Inhalte der unterschiedlichen Parteien vergleichen und seine Wahlentscheidung aufgrund sachlicher Argumente treffen, d.h. bei einer Wahl seine Stimme für diejenige Partei als Interessenvertretung abzugeben, die hinsichtlich deren Inhalte und Aussagen seinen politischen Vorstellungen insgesamt am ehesten entspricht. Dabei ist

der Einfluss des Wählers auf die Politik durch seine rationale Wahlentscheidung $[\ldots]$ um so größer, je genauer die Parteien ihre Ziele dem Wähler zum Zweck des Vergleichs ausgewiesen haben, je mehr sie also, anstatt bloßes Vertrauen zu fordern oder gar durch tönende, aber inhaltsleere Formeln Vertrauen zu erschleichen [...], Informationen für eine rationale Entscheidung zur Verfügung gestellt haben. ${ }^{234}$

Diese Überlegungen können als außersprachliche Motive betrachtet werden, die wiederum in direktem Bezug zu den allgemeinen politischen Zielbestrebungen einer Partei bzw. deren Programm stehen, insbesondere hinsichtlich der beabsichtigten Werbeeffekte von Wahlprogrammen. Somit unterscheiden sich die Rezipienten von Wahlprogrammen grundlegend von denen, die ein Grundsatzprogramm lesen. Wie bereits mehrfach angedeutet kommt den Massenmedien eine entscheidende Funktion zu, denn während die Leser von Wahlprogrammen als potentielle Wähler über die Massenmedien in meist unmittelbarem zeitlichen Bezug zu einer Wahl über die Inhalte informiert werden, sind die Leser von Grundsatzprogrammen als Fachleute und

\footnotetext{
232 Klein 1996b, S. 10

233 Flohr 1968, S. 23

234 Flohr 1968, S. 22
} 
Spezialisten unabhängig von gegenwärtigen Wahlereignissen an den jeweiligen Inhalten und deren Formulierungen interessiert.

In diesem Zusammenhang spielen die jeweils anderen Parteien als politische Gegner eine entscheidende Rolle, da diese „zu den aufmerksamsten Lesern des Programms gehören “235; bei der Formulierung aller Punkte eines Programms muss der Leser mit böswilliger Absicht stets mitberücksichtigt werden, denn

entgegen der sonst gültigen Regel, daß ein Autor auf die Kooperationsbereitschaft, auf den guten Willen also, seiner Leser bauen kann, ja bauen muß, wenn er verstanden werden will, haben die Autoren eines Parteiprogramms die Gewißheit, daß ein Teil ihrer Leser sie wird mißverstehen wollen. ${ }^{236}$

Hinsichtlich der Konzeption bedeutet diese Tatsache, dass bei allen formulierten Aussagen eine Gegenprobe auf mögliche Missverständlichkeiten aus Sicht des politischen Gegners erfolgen muss, um die Formulierungen so unangreifbar wie möglich zu gestalten; selbst Nuancen in der sprachlichen Formulierung können ungewollte Interpretationen des politischen Gegners nach sich ziehen;

aber mehr noch als bei anderen Texten kommt es [...] bei einem solchen Programm nicht nur darauf an, mancherlei erwünschte - auch widersprüchliche - Effekte zu bewirken, sondern auch mancherlei unerwünschte Effekte um jeden Preis zu vermeiden. ${ }^{237}$

Auf diese Tatsache wird an späterer Stelle dieser Untersuchung erneut und ausführlicher eingegangen, wenn es um die Analyse der Leerformeln geht.

\subsubsection{Expliziter Adressatenbezug}

Damit der Inhalt des Programms genügend viele Wähler anspricht, muß sich die Partei in einer pluralistischen Gesellschaft wie der Bundesrepublik darauf einrichten, vielen Bevölkerungsschichten mit verschiedener Einstellung annehmbar zu erscheinen. ${ }^{238}$

Die kulturell und sozial unterschiedlichen Schichten der Gesellschaft müssen im Programm erwähnt werden um eine breitgestreute Rezeption in allen oder möglichst vielen Wähler- und Gesellschaftsschichten zu erzielen. Dabei ist jedoch zu bemerken, dass sich Randparteien mit ihrer Programmatik bewusst ausschließlich an jeweils bestimmte Schichten

\footnotetext{
235 Hermanns 1989, S. 117

236 Hermanns 1989, S. 118

237 Hermanns 1989, S. 115

238 Flohr 1968, S. 53
} 
der Gesellschaft wenden. Die Bedeutung, die eine Partei einer bestimmten gesellschaftlichen Gruppierung gegenüber einräumt, scheint durch die Erwähnung bzw. Nicht-Erwähnung zum Ausdruck zu kommen:

Wir können uns ein demokratisches Staatswesen nicht vorstellen, bei dem die Arbeiter eine so geringe Rolle spielen, daß die Regierungserklärung das Wort ,Arbeiter' nicht einmal erwähnt hat, und wir können uns einen funktionierenden sozialen Organismus auch nicht recht vorstellen, bei dem die Gewerkschaften unerwähnt bleiben. ${ }^{239}$

Die Äußerung des damaligen SPD-Parteivorsitzenden Kurt Schumacher vom 21. September 1949 während der 6. Sitzung im Deutschen Bundestag muss in oben erläutertem Sinn verstanden werden, da sie die Wichtigkeit der expliziten Erwähnung potentieller Adressaten verdeutlicht. Dieser Herleitung folgend lässt sich nun folgende Regel für die politische Sprachverwendung formulieren:

Unterläßt man es, sich zu einer potentiellen Wählergruppe oder zu deren vorrangigen politischen Forderungen zu äußern, so ist damit zu rechnen, daß der politische Gegner und/oder die betreffende Gruppe den Vorwurf erhebt, die Partei interessiere sich nicht für die Anliegen dieser Gruppe oder sie sei ihr nicht gut gesonnen. ${ }^{240}$

Diese Aussage ist insbesondere im Hinblick auf die Differenzierung zwischen Rand- und Volksparteien interessant, da eine Volkspartei grundsätzlich bestrebt ist, ein möglichst breites Spektrum sozialer Gruppen und gesellschaftlicher Schichten zu erreichen, während sich Randparteien bewusst auf gesellschaftliche Nischen konzentrieren. Wenn die SPD als Volkspartei auftritt, dann ist sie

darauf angewiesen, ihre Politik und schon ihr Programm auf mehrere Gruppen abzustellen. Das zwingt sie, viele Sonderinteressen in ihrem Programm zu berücksichtigen; es zwingt sie aber nicht, alle deklarierten Ziele zu einer geschlossenen Konzeption zu verschmelzen, zu einem konsistenten Programm. ${ }^{241}$

Mit anderen Worten: Möglichst viele Rezipienten mit unterschiedlichen Orientierungen sollen angesprochen werden, was die Verwendung von Ausdrücken mit hoher begrifflicher Vagheit sowie die an einen Werbestil angelehnte Abhandlung nennenswerter Punkte ohne inhaltlichen Bezug zur Folge hat. So läuft auch das Godesberger Programm als Programm einer Volkspartei Gefahr, durch die Erwähnung möglichst vieler denkbarer Empfänger den Eindruck einer numerischen Aufzählung zu erwecken. Folgende Wählergruppen sowie Gesellschaftsschichten werden im

239 Verhandlungen des Deutschen Bundestages. Stenographische Berichte. 1. Wahlperiode 1949. Bonn 1950, S. 32

240 Klein 1996a, S. 203

241 Flohr 1968, S. 95 
Godesberger Programm explizit erwähnt: Bürger (6), Staatsbürger (3) und Wirtschaftsbürger (1); die Massenmedien bestehend aus Presse (1), Rundfunk (2), Fernsehen (2), Film (1); Soldat (2), Streitkräfte (2) und Zivilbevölkerung (1), Großunternehmen (2), Arbeitnehmer (7), Verbraucher (3), Unternehmen (7), Betriebe (2) und Familienbetriebe (1), Landbevölkerung (1), Ernährer (1), Kriegsbeschädigte (1) und Kriegshinterbliebene (1); Familie (7), Mann (2), Frau (6), Eltern (1), Mütter (2), Jugend (6) und Kinder (1); Erwachsene (1), Kirchen (2) und Religionsgemeinschaften (2). Auch die Sozialisten (3) sind dabei als Adressaten- und Bezugsgruppe ebenso zu nennen.

Auch Berufsgruppen werden explizit genannt: Richter (3), Bauern (1), Beamte (1), Manager (1), Arzt (1), Ärzte (1), Hausfrauen (2), Studierende (2), Schüler (1), Lehrer (1), Arbeiter (6) und Angestellte (4).

Indirekte Adressatengruppen werden beispielsweise durch die Ausdrücke Minderheiten und Mehrheiten angesprochen. Zwar werden diese Ausdrücke im Godesberger Programm nicht weiter spezifiziert, es ist allerdings anzunehmen, dass der Rezipient, der einer Minderheit - gleichgültig welcher - angehört sich mit dieser Gesellschaftsgruppierung identifiziert; folglich fühlt sich der Rezipient angesprochen und bezieht die formulierten Aussagen auf sich selbst. Die GRÜNEN widmen den nationalen Minderheiten ein eigenes Kapitel Soziale Randgruppen:

(36) (a) Neben dem Recht auf Selbstverwirklichung nationaler Minderheiten, die u.a. ihre kulturellen und religiösen Eigenarten auch durch eine Selbstverwaltung erhalten sollten, treten wir ein gegen die Benachteiligung von Ausländern, Behinderten und Kranken, die aus der Gesellschaft ausgegliedert werden und lediglich als Kostenfaktor Berücksichtigung finden und wie die alten Menschen in Heime abgeschoben werden. Homosexuelle werden in allen Bereichen des Lebens diskriminiert und ebenso wie ehemalige Strafgefangene und Roma/Sinti (,Zigeuner') in Außenseiterpositionen gedrängt. (GRÜNE 1980, Kap. V, 5)

Die Ausdrücke Bund (1), Länder (4), Gemeinden (3), Verbände (3) und Gewerkschaften (6) müssen hinsichtlich ihrer Erwähnung als Adressatengruppe ebenfalls differenziert betrachtet werden. Auffällig ist die außerordentlich positive Bewertung dieser Ausdrücke im Godesberger Programm, was sich als Abgrenzung zur zentralistischen Organisationsstruktur des Kommunismus deuten lässt:

(37) (a) Die Gliederung der öffentlichen Gewalt in Bund, Ländern und Gemeinden soll die Macht verteilen, die Freiheit stärken und dem Bürger durch Mitbestimmung und Mitverantwortung vielfachen Zugang zu den Institutionen der Demokratie geben. Freie Gemeinden sind unerläßlich für eine lebendige Demokratie. [...]

(b) Die Verbände, in denen sich Menschen der verschiedenen Gruppen und Schichten zu gemeinsamen Zwecken zusammenschlie- 
ßen, sind notwendige Einrichtungen der modernen Gesellschaft. (Godesberger Programm 1959, S. 72)

(c) In der heutigen Wirtschaft sind die Arbeitnehmer denen ausgeliefert, die die Kommandostellen der Unternehmen und ihrer Verbände besetzen, wenn sie ihnen nicht in unabhängigen Gewerkschaften ihre solidarische, demokratisch geordnete Kraft entgegenstellen, um die Arbeitsbedingungen frei vereinbaren zu können. (Godesberger Programm 1959, S. 78)

Die oben angeführten Textauszüge zeigen anschaulich, dass die SPD Zusammenschlüsse von Menschen zur Erreichung politischer, wirtschaftlicher oder persönlicher Ziele begrüßt und positiv bewertet. Die große Aufgabe der Gewerkschaften besteht in der Wahrung des Rechts der Erwerbstätigen auf Mitbestimmung im wirtschaftlichen und sozialen Leben; im Godesberger Programm sieht die SPD die Gewerkschaften als Vertreter aller arbeitenden Menschen und als wesentliche Träger des ständigen Demokratisierungsprozesses. In diesem Zusammenhang finden sich auch die Ausdrücke Wohlfahrtsverbände (1) sowie Arbeitnehmer- und Arbeitgeberverbände (1), denen laut Godesberger Programm beim Abschluss von Tarifverträgen größtmögliche Autonomie zugebilligt werden soll; so richtet sich die Erwähnung insbesondere des letzten der Ausdrücke gleichermaßen an die Mitglieder dieser Verbände als auch an den erwerbstätigen Teil der Gesellschaft allgemein.

Der Vollständigkeit halber ist allerdings zu erwähnen, dass der Ausdruck Verbände ebenfalls in einer negativen Bewertung verwendet wird, wenn solche Zusammenschlüsse und Interessengruppen zuviel Macht besitzen:

(38) (a) Mit ihrer durch Kartelle und Verbände noch gesteigerten Macht gewinnen die führenden Männer der Großwirtschaft einen Einfluß auf Staat und Politik, der mit demokratischen Grundsätzen nicht vereinbar ist. [...]

(b) Staat und Gesellschaft dürfen nicht zur Beute mächtiger Interessengruppen werden. (Godesberger Programm 1959, S. 75)

Neben der Möglichkeit der Erwähnung bestimmter Adressatengruppen besteht weiterhin die Möglichkeit, einen gruppenübergreifenden Adressatenbezug durch integrierende den Rezipienten einbeziehende Formulierungen herzustellen; solche Formulierungen können als Quantorenphrasen betrachtet werden, das Indefinitpronomen übt dabei die Funktion des „Allquantors“ oder des „Totalisators“ aus: 
Ist das Bezugsobjekt (logisch) mit dem Allquantor/Totalisator QUANTIFIZIERT, so ist die Gesamtmenge oder Summe aller Elemente der Klasse gemeint, zu der das Bezugsobjekt gehört, $[\ldots] .{ }^{242}$

Die Indikation der Gesamtmenge erfolgt durch die Verwendung des Indefinitpronomens jeder in den Äußerungen (a) bis (f); in den Äußerungen (d), (e) und (f) wird das Pronomen substantivisch gebraucht. Im Godesberger Programm lassen sich folgende Formulierungen nachweisen; die jeweiligen Ausdrücke sind unterstrichen:

(39) (a) Die Sozialisten erstreben eine Gesellschaft, in der jeder Mensch seine Persönlichkeit in Freiheit entfalten und als dienendes Glied der Gemeinschaft verantwortlich am politischen, wirtschaftlichen und kulturellen Leben der Menschheit mitwirken kann. (Godesberger Programm 1959, S. 69)

(b) $\quad$ Eeder Bürger hat die Überzeugung seiner Mitmenschen zu achten. (Godesberger Programm 1959, S. 71)

(c) Ieder Bürger hat im Alter, bei Berufs- oder Erwerbsunfähigkeit oder beim Tode des Ernährers Anspruch auf eine staatliche Mindestrente. (Godesberger Programm 1959, S. 79)

(d) Das gleiche Lebensrecht aller Menschen ist auch dadurch zu verwirklichen, daß bei Krankheit jeder unabhängig von seiner Wirtschaftlichen Lage einen unbedingten Anspruch auf alle dem Stande der ärztlichen Wissenschaft entsprechenden Heilmaßnahmen hat. (Godesberger Programm 1959, S. 79)

(e) Jeder hat ein Recht auf eine menschenwürdige Wohnung. (Godesberger Programm 1959, S. 80)

(f) Jeder, Mann und Frau, ist aufgerufen, hier und in allen Ländern der Erde. (Godesberger Programm 1959, S. 87)

(g) $[\ldots]$ daß der Mensch die Produktivkräfte aufs höchste entwickelte, ungeheure Reichtümer ansammelte, ohne allen einen gerechten Anteil an dieser gemeinsamen Leistung zu verschaffen; [...]

(h) $[\ldots]$ daß der Mensch im atomaren Zeitalter sein Leben erleichtern, von Sorgen befreien und Wohlstand für alle schaffen kann [...]. (Godesberger Programm 1959, S. 68)

(i) Ziel sozialdemokratischer Wirtschaftspolitik ist stetig wachsender Wohlstand und eine gerechte Beteiligung aller am Ertrag der Volkswirtschaft [...]. (Godesberger Programm 1959, S. 73)

(j) Sie will die Kräfte, die durch die industrielle Revolution und durch die Technisierung aller Lebensbereiche entbunden wurden, in den Dienst von Freiheit und Gerechtigkeit für alle stellen. (Godesberger Programm1959, S. 86) 
Weiterhin kann durch die Verwendung des Wortes alle eine Gesamtmenge bezeichnet werden, wie in Äußerung (d) zu sehen. Die Verwendung von alle in den Äußerungen (g) bis (j) unterscheidet sich von allen anderen Verwendungen insofern, als dass das Bezugsobjekt bei diesen Äußerungen fehlt und sich - statt explizit genannt zu werden - implizit erschließt (gemeint sind alle Menschen oder alle Bürger der Gesellschaft). Folgende Bezugsobjekte werden explizit genannt: alle Menschen (7), alle Völker (4), alle Nationen (2), alle Lebensbereiche (2), alle Schulen (2), alle Begabungen (2) sowie in einmaliger Verwendung alle Arbeiter, Angestellte, Beamte, arbeitenden Menschen, kulturwillige Kräfte, Bürger, soziale Geldleistungen, Erziehung, Rechte und Vorrechte, Gebiete, demokratische Kräfte, Probleme, Interessen, Länder, Beteiligte, Möglichkeiten, Heilmaßnahmen, Überlegungen, Stufen der Entwicklung und Bereiche der Kunst. Die Sonderformen alle, die guten Willens sind und alle, für die Freiheit und Menschenwürde, Gerechtigkeit und soziale Sicherheit die Grundlagen der menschlichen Gesellschaft sind sollen in dieser Aufzählung nicht unerwähnt bleiben.

Durch die Verwendung der Wörter alle und jeder mit dem dazugehörigen Bezugsobjekt soll zum Ausdruck gebracht werden, dass die SPD sich ausnahmslos auf die Gesamtheit der jeweiligen Gruppe oder Klasse bezieht, d.h. ohne jeweils bestehende Unterschiede zu berücksichtigen; die Verwendung der beiden Wörter ohne Bezugsobjekt bringt eine „Entpersönlichung des Ausdrucks“ ${ }^{\prime 243}$ mit sich und kann zu inhaltsarmen Leerformeln (Wohlstand für alle in (h)) führen.

\subsection{Das Personalpronomen wir}

An dieser Stelle soll auch auf die parteiübergreifende Verwendung des Personalpronomens wir (1. Person Plural) als "Wir-Deiktika“244 hingewiesen werden. Dabei wird grundsätzlich von der Tatsache ausgegangen, dass der Sprecher bzw. der Produzent der sprachlichen Handlung bei der Verwendung von wir sich selbst zu der betreffenden Gruppe rechnet: ${ }^{245}$ "Wir bedeutet zunächst und eigentlich: ,der Sprecher und die

243 v. Polenz 1985, S. 34/35

244 Volmert, Johannes: „'While the world prayed for peace, Saddam prepared for war.' Bushs Fernsehrede zur Eröffnung des Bombenkriegs gegen den Irak." In: Inszenierte Information. Politik und strategische Kommunikation in den Medien. Hg. v. Adi Grewenig. Opladen 1993, S. 213

245 Der mögliche Extremfall, dass der Sprecher selbst aus der betreffenden Gruppe ausgeschlossen ist, kann in den Parteiprogrammen nicht nachgewiesen werden und ist vielmehr im Umgang Erwachsener mit Kindern geläufig (Krankenschwester-Wir oder paternalistisches Wir). 
Angehörigen seiner Gruppe.'“246 BURKHARDT (2003) formuliert folgende Thesen:

In der politischen Rede enthält das wir zumeist in dem Sinne eine Anmaßung, als es die (nicht immer gerechtfertigte) Präsupposition eines Sprechers im Namen anderer einschließt. Es ist unterschwellig appellativ und soll zumeist nach innen gruppenintegrativ, nach außen aus- und abgrenzend wirken. Vor allem im politischen Sprechen besteht dabei die Gefahr, die Eigengruppe zum kollektiv-uniformierten ,wir' zu hypostasieren und der andersartigen Fremdgruppe abwehrend gegenüberzustellen. ${ }^{247}$

Bei Betrachtung des Ausdrucks wir als Zeigewort in den Parteiprogrammen kann daher weiterhin festgestellt werden, dass im Einzelnen unterschiedliche Gruppen-Denotate bezeichnet werden, die über die Gruppe des Sprechers bis zu einer den Sprecher einschließenden Gesamtheit hinausreichen. Jedoch erschließen sich die unterschiedlichen Bedeutungen nur unter Berücksichtigung des jeweiligen Kontextes, der im Folgenden gegebenenfalls zitiert werden soll. So soll nachfolgend der Versuch unternommen werden, unterschiedliche Wir-Kollektive nachzuweisen und zu kategorisieren, obwohl es "zur Technik dieser WirAppelle gehört, daß Größe und Grenzen der bezeichneten WirKollektive bewußt unscharf gelassen werden. ${ }^{\text {}} 248$

\section{A Anthropologisches wir}

Die Kategorie des anthropologischen wir umfasst Aussagen, die das Personalpronomen wir dem Schema "der Sprecher und die Gesamtheit seiner Mitmenschen“249 folgend verwenden. Die Äußerungen, die dieser Bedeutungsmöglichkeit folgen, reichen in ihrer Aussage und ihrem Bezug über die Parteigrenzen hinaus und haben häufig appellativen Charakter, wie beispielsweise nachfolgend zitierte Äußerung (a). Insbesondere die Äußerung (c) macht die Bedeutung von wir in dieser Verwendung deutlich, denn für die von den GRÜNEN dargestellten negativen Begleiterscheinungen der Zivilisation ist nicht die Partei, sondern sind alle Menschen verantwortlich. Wir bezieht sich in (c) somit auf jedes einzelne Mitglied in Industrienationen: "Jeder kann und jeder soll sich als Glied der ingroup angesprochen fühlen; die Aussagen signalisieren, daß der Sprecher [...] keine Parteien und Fraktionen, keine Rassen und Schichten mehr kennt. ${ }^{\prime 250}$ Die von der SPD formulierte Äußerung (d) hat einen

\footnotetext{
246 Burkhardt 2003, S. 406

247 Burkhardt 2003, S. 406

248 Volmert 1993, S. 215

249 Burkhardt 2003, S. 411

250 Volmert 1993, S. 215
} 
appellativen Charakter und beschreibt Grundvoraussetzungen für bestimmte anstrebenswerte gesellschaftliche Verhältnisse, die über die Bundesrepublik Deutschland hinaus gültig sind.

(40) (a) Diesen Widerspruch aufzulösen, sind wir Menschen aufgerufen. (Godesberger Programm 1959, S. 69)

(b) Wir befinden uns am Beginn einer neuen Phase der Industriegesellschaft. (CSU 1968, S. 240)

(c) Die ökologische Weltkrise verschärft sich von Tag zu Tag: Die Rohstoffe verknappen sich, Giftskandal reiht sich an Giftskandal, Tiergattungen werden ausgerottet, Pflanzenarten sterben aus, Flüsse und Weltmeere verwandeln sich in Kloaken, der Mensch droht inmitten einer späten Industrie und Konsumgesellschaft geistig und seelisch zu verkümmern, wir bürden den nachfolgen den Generationen eine unheimliche Erbschaft auf. [...] Erst in dem Maße, wie wir uns von der Überschätzung des materiellen Lebensstandards freimachen, wie wir wieder die Selbstverwirklichung ermöglichen und uns wieder auf die Grenzen unserer Natur besinnen, werden auch die schöpferischen Kräfte frei werden für die Neugestaltung eines Lebens auf ökologischer Basis. [...] Wir dürfen die Stabilität der Ökosysteme nicht zerstören. [...] Obwohl wir zweifellos alle in einer Welt leben, so widerspräche es doch völlig den Grundsätzen einer ökologischen Politik, wenn man alle Probleme einheitlich und zentralistisch lösen wollte. (GRÜNE 1980, Kap. I/Kap. III, 1)

(d) Wer Bewahrenswertes erhalten will, muß verändern: Wir brauchen einen Fortschritt, der den Frieden nach innen und außen sichert, das Leben von Mensch und Natur bewahrt, Angst überwindet und Hoffnung weckt. (SPD 1989, S. 7)

\section{B Inklusives wir}

Bei der Kategorie des inklusiven wir handelt es sich um Äußerungen nach dem Schema "der Sprechers und seine Gruppe “251; dabei wird dem Hörer das Gefühl vermittelt, er sei Teil einer mit wir angesprochenen Gruppe, was dazu dienen kann, den Hörer für die Wir-Gruppe zu vereinnahmen. Bei der Verwendung von wir nach diesem Schema werden die von der Partei geplanten Vorhaben und Handlungsabsichten beschrieben oder wie in Äußerung (e) die Umstände der Parteientstehung. Der Sprecher solcher Äußerungen rechnet sich dabei selbst zu der betreffenden Gruppe; die Trennung der Kategorien erscheint im Hinblick auf die Äußerung (f) erschwert, da hier die Bewertung der Verwendung von wir als kollektivem wir ebenfalls möglich scheint.

(e) Wir streiten für die Demokratie. Sie muß die allgemeine Staats- und Lebensordnung werden, weil sie allein Ausdruck der 
Achtung vor der Würde des Menschen und seiner Eigenverantwortung ist. (Godesberger Programm 1959, S. 70)

(f) Hervorgegangen sind wir aus einem Zusammenschluss von grünen, bunten und alternativen Listen und Parteien. Wir fühlen uns verbunden mit all denen, die in der neuen demokratischen Bewegung mitarbeiten: den Lebens-, Natur- und Umweltschutz- verbünden, den Bürgerinitiativen, der Arbeiterbewegung, christlichen Initiativen, der Friedens- und Menschenrechts-, der Frauen- und 3.-Welt-Bewegung. Wir verstehen uns als Teil der grünen Bewegung in aller Welt. [...] $\underline{\text { Wir }}$ halten es für notwendig, die Aktivitäten außerhalb des Parlaments durch die Arbeit in den Kommunal- und Landesparlamenten sowie im Bundestag zu ergänzen. Wir wollen dort unseren politischen Alternativen Öffentlichkeit und Geltung verschaffen. Wir werden damit den Bürger- und Basisinitiativen eine weitere Möglichkeit zur Durchsetzung ihrer Anliegen und Ideen eröffnen. (GRÜNE 1980, Kap. I)

\section{Nationales wir}

Das nationale wir umfasst Äußerungen mit der Bedeutung „der Sprecher und seine Landsleute. "252 Wir steht in dieser Verwendung für die Nation, die Gemeinschaft oder die Gemeinde. Wenn die NPD in Äußerung (g) eine bestimmte Sozialpolitik fordert, dann gilt diese Forderung für alle Bürger, was durch den Ausdruck ganzes Volk verdeutlicht wird; wenn die Partei weiterhin eine starke militärische Kraft fordert, dann bezieht sich auch diese Forderung nicht nur auf die Partei, sondern auf das Land oder den Staat, d.h. auf das gesamte Gebiet der Bundesrepublik Deutschland.

Der nachfolgend zitierten Textstelle (h) geht im Parteiprogramm der GRÜNEN die Beschreibung der Ungerechtigkeit gegenüber den Völkern der Dritten Welt als Handelspartner voraus. Wir könnte neben der Bundesrepublik Deutschland als Handelspartner aber auch die heutige Europäische Union (im Jahre 1980 noch Europäische Gemeinschaft) beinhalten, im Sinne von: wir als deutsche Bürger zusammen mit unseren europäischen Nachbarstaaten und deren Bürger. Deutlicher wird die Bedeutung bei Betrachtung der Äußerung (i), da die Aussage sich auf den Sprecher, seine Partei und seine Landsleute bezieht. Die zitierte Äußerung (j) wird hier ebenfalls als nationales wir gewertet, da die SED bei ihrer Formulierung von der Bevölkerung der damaligen DDR ausgeht und jeden Bürger somit als Zeugen und Mitgestalter betrachtet; die Aussage schließt die SED und deren Mitglieder dabei mit ein.

Auch die FRAUEN verwenden wir in (k) als nationales wir, wenn auch als Gruppe innerhalb einer Nation geschlechtsspezifisch abgegrenzt und in 
der Bedeutungsreichweite über Deutschland als Nation auf alle Industrieländer ausgedehnt.

(g) Wir brauchen eine Sozialpolitik, die sozialer Gerechtigkeit und volkswirtschaftlicher Vernunft entspricht. Sie hat die Aufgabe, den Wohlstand des ganzen Volkes zu festigen, den einzelnen in allen Wechselfällen des menschlichen Lebens vor unverschuldeter Not zu bewahren und allen Arbeitenden einen sorgenfreien Lebensabend $\mathrm{zu}$ sichern. [...] Da es in Europa keinen Krieg mehr geben darf, benötigen wir eine militärische Kraft, stark genug, jeden möglichen Gegner abzuschrecken. Dieses Ziel ist bei einer gemeinsamen europäischen Kraftanstrengung erreichbar. (NPD 1973, S. 367/371/372)

(h) Dann werden Zahlungen, die wir wie Almosen ausstreuen, weitgehend überflüssig werden. (GRÜNE 1980, Kap. II, 9)

(i) Diese Forderung müssen besonders wir Deutschen erheben, da uns jeder sogenannte ,Ernstfall' den Untergang bringen würde. (GRÜNE 1980, Kap. III, 2)

(j) Der Übergang vom Kapitalismus zum Sozialismus ist Hauptinhalt und grundlegendes Entwicklungsgesetz unserer Epoche. Wir sind Zeugen und Mitgestalter der größten Revolution in der Geschichte der Menschheit. (SED 1963, S. 210)

(k) Indem wir Frauen in den Industrieländern teilhaben an einem Wohlstand, der auf dem Rücken von Frauen und ganzen Völkern in den sogenannten Entwicklungsländern erwirtschaftet wird, sind wir ebenfalls Nutzniesserinnen und Mitverantwortliche in diesem Modell. (DIE FRAUEN 2002, S. 15)

\section{Kollektives wir}

(1) Wir Nationaldemokraten wenden uns gegen die Aufrechterhaltung der heute schon durch die Geschichtsforschung widerlegten Lüge, die unserem Volk die Alleinschuld an zwei Weltkriegen auferlegt. (NPD 1973, S. 373)

(m) Wir GRÜNEN fordern: [...]. Dazu schlagen wir GRÜNEN folgende Maßnahmen vor: [...]. (GRÜNE 1980, Kap. II, 6)

(n) Wir, die Mitglieder der Partei des Demokratischen Sozialis-

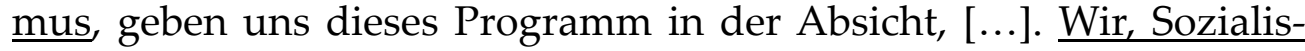
tinnen und Sozialisten, Mitglieder der PDS, wollen am widerstand gegen diese Welt teilnehmen [...]. Über viele Fragen haben wir, die Mitglieder der PDS, unterschiedliche Auffassungen. [...] Wir Mitglieder der Partei des Demokratischen Sozialismus stehen für [...]. Seitdem haben wir, die Mitglieder der PDS, einen anhaltenden Wandlungsprozess innerhalb unserer Partei vorangetrieben [...]. (PDS 2003, S. $1 / 31$ )

(o) Wir Bündnisgrüne unterstützen die Trennung von Kirche und Staat. (B 90/GRÜNE 2002, S. 120) 
(p) Deswegen haben wir die Feministische Partei DIE FRAUEN gegründet. (DIE FRAUEN 2002, S. 6)

Das kollektive wir folgt dem Schema "der Sprecher und die (engere) Gruppe, in deren Kreis er sich stellt. ${ }^{\prime 253}$ Deutlich wird bei Betrachtung der zitierten Textstellen erneut, dass sich diese Kategorie nur schwer von der Kategorie inklusives wir abgrenzen lässt.

Die oben zitierte Wendung der NPD zielt einerseits auf das Hervorrufen eines kollektiven Empfindens ab, andererseits grenzt sich die Partei mit dieser Formulierungen von anderen Parteien ab. Zwar hat die Formulierung auch eine Wirkung, die auf die Parteimitglieder integrativ wirkt, die Äußerung gehört jedoch deshalb nicht der Kategorie integratives wir an, weil Rezipienten außerhalb der Partei nicht integriert werden.

Auch die NSDAP verwendet ihrem Programm von 1920 das Personalpronomen wir. Insgesamt kommt das Wort in der Wendung wir fordern 23-mal vor und gehört somit der Kategorie des kollektiven wir an, da der Bezug eng auf den Sprecher bzw. die Partei beschränkt bleibt.

Eindeutig als kollektives wir verwenden die FRAUEN das Personalpronomen in Äußerung (p), da die Partei nicht von allen Mitgliedern (die Gruppe), sondern nur von einigen wenigen innerhalb dieser Gruppe gegründet wurde; wir bezieht sich in (p) folglich auf den engen Kreis, in den sich auch der Sprecher (als Verfasserkollektiv) stellt.

\section{E Integratives wir}

Das Schema des integrativen wir resultiert aus der Bedeutung „der Sprecher und seine Gruppe gemeinsam mit anderen Gruppen." 254

Beispiele für die Verwendung des integrativen wir lassen sich im Parteiprogramm der FDP, der PDS und der SPD nachweisen. Bei der Formulierung der FDP wird sowohl der Bürger/Wähler (als Rezipient und als Mitglied der Gesellschaft) unmittelbar angesprochen als auch Mitglieder der anderen konkurrierenden oder kooperierenden Parteien, da sich beispielsweise mögliche Folgen der angesprochenen Reformbewegung auch bei den gegnerischen Parteien bemerkbar machen. Die Aussagen richten sich aber dennoch auch an die Partei bzw. deren Mitglieder. Das Wort wir in der nachfolgenden Äußerung (q) umfasst daher wie oben zitiert den Sprecher und seine Gruppe gemeinsam mit anderen (außerpolitischen) Gruppen. Besonders deutlich wird die Einbindung der anderen Parteien, d.h. die integrative Wirkung über die eigenen Parteimitglieder hinaus, am Beispiel der PDS in Äußerung (r). Die SPD bezieht in ihrer Äußerung (s) die Mitglieder anderer Gruppen (demokratischen So-

Burkhardt 2003, S. 411

254

Burkhardt 2003, S. 411 
zialisten aller Länder) in ihre eigene Gruppe (Sozialdemokraten) mit ein; ohne die Erweiterung gemeinsam mit den demokratischen Sozialisten aller Länder ließe sich wir als kollektives wir interpretieren.

(q) Wir stehen heute am Anfang der zweiten Phase einer von der bürgerlichen Revolution ausgehenden Reformbewegung auch in der Gesellschaft, wie sie nicht zuletzt in den tiefgreifenden und nachhaltigen Bewußtseinsveränderungen der weltweiten Jugendrevolte sich ankündigt. [...] Die Gesellschaft sind wir alle! [...] Die Frage, die wir uns auf dem Boden unserer rechtlichen Wirklichkeit stellen können, ist darum ausschließlich die nach den Inhalten und Grenzen solchen Privateigentums, vor allem an Grund und Boden, an Naturschätzen und Produktionsmitteln. [...] Wir brauchen deshalb Beteiligungsformen, die den Vermögenszuwachs unmittelbar und für alle erfassen. (FDP 1971 S. 250/252/254/259)

(r) Wir sind als sozialistische Partei bereit, langfristig an einem Mitte-Links-Bündnis mitzuwirken, das auf solche Veränderungen gerichtet ist. (PDS 2003, S. 32)

(s) Darum wollen wir Sozialdemokraten gemeinsam mit den demokratischen Sozialisten aller Länder für ihn arbeiten. (SPD 1989, S. 7)

\section{F $\quad$ Konfrontatives wir}

Das konfrontative wir folgt dem Schema „der Sprecher und seine Gruppe im Gegensatz zu anderen Gruppen. ${ }^{\prime 255}$ Bei der Verwendung des Personalpronomens nach diesem Schema scheint es in erster Linie darum zu gehen, unter Gegnerbezug die eigene Position zu betonen und somit von den Positionen der gegnerischen Parteien abzugrenzen. Neben der Realisierung der Profilfunktion wirkt die Verwendung nach diesem Schema jedoch auch nach innen gruppenintegrierend. So wird in Äußerung (t) impliziert, dass andere Parteien oder Gruppierungen bestimmten Herrschaftssystemen wie einer Diktatur möglicherweise nicht widerstehen können, und die Aussage in Äußerung (u) macht deutlich, dass sich die GRÜNEN als Partei grundlegend von allen anderen Parteien unterscheiden.

Auch die PDS bringt mit ihrer Äußerung (v) zum Ausdruck, dass sie sich als Partei deutlich von anderen Parteien oder Ideologien distanziert; zwar lässt sich dadurch ebenso eine gruppenidentifikatorische Wirkung bestätigen, es scheint jedoch primär die Konfrontation im Vordergrund zu stehen.

(t) Wir widerstehen jeder Diktatur, jeder Art totalitärer und autoritärer Herrschaft; denn diese mißachten die Würde des Menschen, vernichten seine Freiheit und zerstören das Recht. (Godesberger Programm 1959, S. 70) 
(u) Wir sind die Alternative zu den herkömmlichen Parteien. [...] Wir werden uns nicht an einer Regierung beteiligen, die den zerstörerischen Kurs fortführt. [...] Gegenüber der eindimensionalen Produktionssteigerungspolitik vertreten wir ein Gesamtkonzept. (GRÜNE 1980, Kap. I)

(v) Als demokratische Sozialistinnen und Sozialisten bleiben wir insbesondere jenen Traditionen der deutschen Arbeiterbewegung - der Gewerkschaften, der sozialdemokratischen wie der kommunistischen Parteien - verpflichtet, die in den Kämpfen gegen Nationalismus und Militarismus, gegen Imperialismus und Faschismus entstanden und sich 1945/1946 im gemeinsamen Einsatz für die antifaschistisch-demokratische Neuordnung Deutschlands bewährten. Aus historischer Erfahrung wenden wir uns entschieden gegen jegliche Form von Antisozialdemokratismus und Antikommunismus. [...] Wir leisten Widerstand gegen jede Art von Kriegsbeteiligung seitens der Bundesrepublik. Wir treten Rassismus und Rechtsextremismus entschlossen entgegen. [...] Wir kämpfen für die Überwindung des Kapitalismus, weil wir in einer Gesellschaft von Freiheit, Gleichheit und Solidarität leben wollen. (PDS 2003, S. 31/32/34)

\subsubsection{Impliziter Adressatenbezug}

Die Adressaten eines Parteiprogramms müssen allerdings nicht zwangsläufig explizit im Text erwähnt werden. Die nachfolgend zitierten Textbeispiele wenden sich implizit an die Berufs- oder Wählergruppen der Schöffen (a), der Kriegsdienstverweigerer (b), der Lohn- und Gehaltsempfänger (c), der Stahlarbeiter und Bergbauer (d), der Rentner (e), der Patienten (f), der Sozialleistungsempfänger (g), der Mieter (h), der Wissenschaftler bzw. Forscher (i) und der Künstler (j); die jeweiligen Textstellen sind unterstrichen:

(41) (a) An der Rechtspflege sind ehrenamtliche Richter gleichberechtigt zu beteiligen. (Godesberger Programm 1959, S. 72)

(b) Die Sozialdemokratische Partei Deutschlands stellt sich schützend vor jeden Bürger, der aus Gewissensgründen den Dienst mit der Waffe oder an Massenvernichtungsmitteln verweigert. (Godesberger Programm 1959, S. 73)

(c) Die Lohn- und Gehaltspolitik ist ein geeignetes und notwendiges Mittel, um Einkommen und Vermögen gerechter zu verteilen. (Godesberger Programm 1959, S. 77)

(d) Die Mitbestimmung in der Eisen- und Stahlindustrie und im Kohlenbergbau ist ein Anfang zu einer Neuordnung der Wirtschaft. (Godesberger Programm 1959, S. 78)

(e) Jeder Bürger hat im Alter, bei Berufs- oder Erwerbsunfähigkeit oder beim Tode des Ernährers Anspruch auf eine staatliche Mindestrente. Auf ihr bauen weitere, persönlich erworbene Rentenan- 
sprüche auf. So ist die im Arbeitsleben erreichte Lebenshaltung zu sichern. [...]

(f) Technik und Zivilisation setzen den Menschen einer Vielzahl von gesundheitlichen Gefährdungen aus. [...] Es ist eine öffentliche Aufgabe, die Krankenhausversorgung sicherzustellen. [...] Das gleiche Lebensrecht aller Menschen ist auch dadurch zu verwirklichen, daß bei Krankheit jeder unabhängig von seiner wirtschaftlichen Lage einen unbedingten Anspruch auf alle dem Stande der ärztlichen Wissenschaft entsprechenden Heilmaßnahmen hat. Die freigewählte ärztliche Hilfeleistung wird durch volle wirtschaftliche Sicherung im Krankheitsfalle ergänzt. [...]

(g) Zur Bewältigung besonderer Lebensschwierigkeiten und Notlagen sind die allgemeinen sozialen Leistungen durch individuelle fürsorgerische Dienste und Leistungen der Sozialhilfe zu ergänzen. (Godesberger Programm 1959, S. 79)

(h) Die Wohnungs-, Bau- und Bodenpolitik muß den Mangel an Wohnraum beschleunigt beheben. Der soziale Wohnungsbau ist zu fördern. Der Mietzins ist nach sozialen Gesichtspunkten zu beeinflussen. Die Bodenspekulation ist zu unterbinden, ungerechtfertigte Gewinne aus Bodenverkäufen sind abzuschöpfen. (Godesberger Programm 1959 , S. 80)

(i) Wissenschaftliche Forschung und Lehre müssen frei sein. [...] Ausreichende öffentliche Mittel für Forschung und Lehre müssen zur Verfügung gestellt werden. [...] Von der Förderung wissenschaftlicher Forschung und Lehre darf kein Gebiet der Wissenschaft ausgenommen sein. [...]

(j) Künstlerischem Schaffen ist volle Freiheit zu gewähren. [...] Die künstlerische Entfaltung darf durch kein Reglement, insbesondere durch keine Zensur, beschränkt werden. (Godesberger Programm 1959, S. 83)

Die Aufzählung und Erwähnung aller als Adressaten in Frage kommenden sind jedoch nicht die einzigen Faktoren, die einen Text wie das Godesberger Programm streckenweise langweilig oder zäh erscheinen lassen. Grundsatzprogramme können auch noch aus anderen Gründen langweilig wirken.

\subsubsection{Selbstverständlichkeiten}

Oftmals sind Grundsatzprogramme inhaltlich für nicht fachkundige Leser voller Selbstverständlichkeiten und müssen „unentwegt und unverdrossen wiederholen, wovon fast jedermann sowieso schon überzeugt ist “256. Die Erwähnung von Selbstverständlichkeiten resultiert al-

256 Hermanns 1989, S. 115 
lerdings aus einer immens wichtigen, politischen Laien jedoch meist unbekannten oder unbewussten Regel:

Bekennt man sich in einem Grundsatzprogramm nicht ausdrücklich zu den Überzeugungen, die für alle oder die meisten selbstverständlich sind, so besteht die Gefahr, daß der politische Gegner daraus den Verdacht ableitet, daß man sie nicht teile. ${ }^{257}$

Dennoch besteht die Gefahr, dass bei solchen Formulierungen der Eindruck einer gewissen „Phrasenhaftigkeit“258 entsteht. Diese Aussage leitet den nächsten Untersuchungsschwerpunkt ein.

\subsubsection{Phrasenhaftigkeit in den Parteiprogrammen}

Es soll nun die Begrifflichkeit der Ausdrücke Phrasen, Phraseologismen und Leerformeln beleuchtet werden. Dabei ist anzumerken, dass die populäre Bezeichnung der Phrase in der Forschung umstritten oder gar zu vermeiden ist, da die Bezeichnung "terminologisch unscharf ist und ihr eine $\mathrm{zu}$ negative Konnotation ${ }^{259}$ anhaftet.“260 Wenn in den folgenden Untersuchungen von Phrasen oder phrasenhaften Formulierungen gesprochen wird, dann sind damit meist zwar populäre, jedoch inhaltsarme oder inhaltsleere Ausdrücke (Leerformeln) gemeint.

\subsubsection{Phraseologismen}

Unter Phraseologismen versteht man allgemein

Wortverbindungen, die aus mindestens zwei Wörtern, maximal jedoch einem ganzen Satz bestehen. Diese Wortverbindungen sind relativ feste Einheiten, die - unterhalb der Satzebene - wie Lexeme benutzt werden. Sie können zudem idiomatisch sein. ${ }^{261}$

257 Klein 1996a, S. 203

258 Klein 1996a, S. 203

259 Auch die Verwendung des Ausdrucks Konnotation ist in der Forschungsliteratur umstritten. Im Beispiel hier wird unter der konnotativen Bedeutung des Wortes Phrase eine über die rein sachliche Kernbedeutung hinausreichende wertende (subjektive, assoziative, emotionale oder individuelle) Bedeutungskomponente verstanden, die zusammen mit dem kontext- und situationsunabhängigen begrifflichen Inhalt (der so genannten denotativen Bedeutung oder Denotat) die Gesamtbedeutung des Wortes ausmacht.

260 Elspaß, Stephan: „Phraseologie im deutschen Parlamentarismus. Zu historischen Entwicklungen im Sprachverhalten politischer Redner." In: Sprache des deutschen Parlamentarismus. Hg. v. Armin Burkhardt/Kornelia Pape. Wiesbaden 2000, S. 263

261 Elspaß 2000, S. 263 
Merkmale von Phraseologismen sind offensichtlich Polylexikalität und Festigkeit, d.h. die jeweiligen phraseologischen Wendungen bestehen aus lexikalischen oder syntaktischen Einheiten. Eine ausführlichere Definition, die den Bezug zu den phrasenhaften Verwendungsweisen in der politischen Sprachverwendung und speziell im Godesberger Programm deutlich macht, beschreibt einen Phraseologismus als

eine Verbindung von zwei oder mehr Wörtern dann, wenn (1) die Wörter eine durch die syntaktischen und semantischen Regularitäten der Verknüpfung nicht voll erklärbare Einheit bilden, und wenn (2) die Wortverbindung in der Sprachgemeinschaft, ähnlich wie ein Lexem, gebräuchlich ist. Die beiden Kriterien stehen in einem einseitigen Bedingungsverhältnis: Wenn (1) zutrifft, dann auch (2), aber nicht umgekehrt. ${ }^{262}$

\subsubsection{Idiome}

Kennzeichnend für eine idiomatische Wendung ist die Differenz zwischen lexikalischer und aktueller Bedeutung. Ein idiomatischer Ausdruck kann als komplexe lexikalische Einheit und als Teilbereich der Phraseologie (als Gesamtbereich der Phraseologismen) verstanden werden: „Für den Gesamtbereich der phraseologischen Einheiten steht der Terminus ,Phraseologismus', für die wichtigste Teilklasse der Terminus ,Idiom'. "263 Für diese Teilklasse ergibt sich folgende Definition:

Diejenige Teilklasse der Phraseologismen, deren Komponenten eine durch die syntaktischen und semantischen Regularitäten der Verknüpfung nicht voll erklärbare Einheit bilden (= Merkmal der ,Idiomatizität'), bilden den Bereich der ,Idiome'.264

Die nachfolgend aufgelisteten Beispielsätze des Godesberger Programms sollen auf ihren phraseologischen/idiomatischen Gehalt hin untersucht werden; Kandidaten für idiomatische Wendungen sind unterstrichen:

(42) (a) Diesen Widerspruch aufzulösen, sind wir Menschen aufgerufen. In unsere Hand ist die Verantwortung gelegt für eine glückliche Zukunft oder für die Selbstzerstörung der Menschheit. (Godesberger Programm 1959, S. 69)

(b) Mehr als ein Drittel des Sozialprodukts geht auf diese Weise durch die öffentliche Hand. $[\ldots .$.

262 Burger, Harald/Buhofer, Annelies/Sialm, Ambros: Handbuch der Phraseologie. Berlin/New York 1982, S. 1

263 Burger, Harald: „Besondere Formen lexikalischer Einheiten I: Phraseologismen." In: Lexikologie. Ein internationales Handbuch zur Natur und Struktur von Wörtern und Wortschätzen (1. Halbband). Hg. v. D. Alan Cruse/Franz Hundsnurscher/Michael Job/Peter Rolf Lutzeier. Berlin/New York 2002, S. 392

264 Burger 2002, S. 392 
(c) Deshalb bejaht die Sozialdemokratische Partei den freien Markt, wo immer wirklich Wettbewerb herrscht. (Godesberger Programm 1959, S. 74)

(d) Eine weitgehende Publizität muß der Öffentlichkeit Einblick in die Machtstruktur der Wirtschaft und in die Wirtschaftsgebarung der Unternehmen verschaffen, damit die öffentliche Meinung gegen Machtmißbrauch mobilisiert werden kann. [...]

(e) Jede Zusammenballung wirtschaftlicher Macht, auch die in Staatshand, birgt Gefahren in sich. (Godesberger Programm 1959, S. 76)

(f) Deshalb können sie auch nicht den Anspruch der jungen Staaten auf solidarische Hilfe erfüllen, die eben das Joch der kolonialen Ausbeutung abschütteln und die ihre nationale Zukunft in Freiheit aufbauen und am Wohlstand der Welt teilnehmen wollen. [...]

(g) Auf dem Rücken ihrer Völker errichten sie eine wirtschaftliche und militärische Macht, die zur wachsenden Bedrohung der Freiheit wird. (Godesberger Programm 1959, S. 86)

(h) Auf deutschem Boden sammeln sich die Sozialisten in der Sozialdemokratischen Partei Deutschlands, die jeden in ihren Reihen willkommen heißt, der sich zu den Grundwerten und Grundforderungen des demokratischen Sozialismus bekennt. (Godesberger Programm 1959, S. 87)

(i) Die Sozialdemokratische Partei Deutschlands bekennt sich zur Verteidigung der freiheitlich-demokratischen Grundordnung. [...] Die Bundesrepublik Deutschland darf atomare und andere Massenvernichtungsmittel weder herstellen noch verwenden. (Godesberger Programm 1959, S. 72/73)

(j) Entwicklungsländer haben Anspruch auf die Solidarität der anderen Völker. [...]

(k) $\quad \mathrm{Zu}$ Unrecht berufen sich die Kommunisten auf sozialistische Traditionen. In Wirklichkeit haben sie das sozialistische Gedankengut verfälscht. (Godesberger Programm 1959, S. 70)

(1) Wer nicht über gleiche Macht verfügt, hat nicht die gleiche Entfaltungsmöglichkeit, er ist mehr oder minder unfrei. [...]

(m)Durch solche Unternehmen soll den Interessen der Allgemeinheit Geltung verschafft werden. (Godesberger Programm 1959, S. 75)

(n) Auch dort wächst das Freiheitsstreben, das keine Herrschaft auf dieDauer völlig niederhalten kann. (Godesberger Programm 1959, S. 86) 


\section{$\underline{\mathrm{Zu}}$ (a) und (g)}

Der Ausdruck in unsere Hand gelegt bedeutet in Äußerung (a), dass die Verantwortung an alle Menschen übertragen wird. Die Verantwortung liegt dann in den Händen der Menschen. Es handelt sich bei dem unterstrichenen Ausdruck in (a) folglich um ein etabliertes Mehr-WortElement; die Wendung etwas in jemandes Hand/Hände legen ist in PONS (1993) ${ }^{265}$ gelistet und wird auch als ,verbale phraseologische Ganzheit “266 bezeichnet, die sich durch die Merkmale „Lexikalisierung, Stabilität und Vollidiomatizität" auszeichnet.

Die voll-idiomatische Wendung etwas auf dem Rücken von jemandem tun in Äußerung (g) kann als verbale phraseologische Ganzheit betrachtet werden. Durch die Verwendung dieser pathetisch wirkenden Formulierung betont die SPD ihre Abneigung gegenüber den kommunistischen Machthabern.

\section{$\underline{\mathrm{Zu}(\mathrm{b})}$}

Der aus dem Rechtswesen stammende Ausdruck öffentliche Hand in Äußerung (b) ist „im engeren Sinn gleichbedeutend mit Fiskus.“267 Es scheint sich hierbei um eine idiomatische und populäre Verwendung vor allem im finanzpolitischen Bereich zu handeln, da Fiskus als Bezeichnung für den Staat als Vermögensträger, d.h. als Eigentümer von Vermögen, verstanden wird. Für den Ausdruck öffentliche Hand als idiomatische Wendung spricht, dass der Ausdruck mental als lexikalische Einheit - einem Wort ähnlich - gespeichert, abgerufen und produziert werden kann und zwar im Sinn von: "die öffentliche Verwaltung als am Wirtschaftsverkehr teilnehmender Vermögensträger, z.B. als Arbeitgeber, Auftraggeber. “268 Wie verhält sich nun aber die jeweils freie Bedeutung der Komponenten zur idiomatischen Bedeutung des Ausdrucks? Wenn ein bestimmter Teil eines Staatsvermögens aus Steuergeldern besteht, dann ist damit eine Geldmenge gemeint, die durch die Hände der Öffentlichkeit, d.h. aller Bürger, dem Staat zugeflossen ist. Dieser verwaltet danach die Gelder als öffentliche Hand. Die Bedeutung des Ausdrucks öffentliche Hand ist nicht unmittelbar aus den Bedeutungen der freien Komponenten $\mathrm{zu}$ erschließen, da öffentlich in seiner Bedeutung zwar konstant bleibt, die Bedeutung der Komponente Hand ist jedoch semantisch transformiert. Daher scheint es sich bei dem Ausdruck öffentliche

265 PONS - Deutsche Idiomatik 1993, S. 311

266 Phraseologismen in ausgewählten Bundestagsdebatten. Opladen/Wiesbaden 1998, S. 70 
Hand um eine teil-idiomatischen Wendung zu handeln: "Als ,teilidiomatisch' bezeichnet man Ausdrücke, bei denen eine Komponente ihre freie Bedeutung beibehält, die andere eine von der freien abweichenden Bedeutung hat [...]." ${ }^{269}$ Der Ausdruck öffentliche Hand wird sowohl im DUDEN (1999)270 als auch im PONS (1993) ${ }^{271}$ als formelhafter oder formeller idiomatischer Ausdruck mit der oben dargestellten Bedeutung gelistet.

\section{$\underline{\mathrm{Zu}}$ (c)}

Der Ausdruck freier Markt gehört zur Kategorie der "phraseologischen Termini“; diese Termini ",sind in ihrer Bedeutung strikt festgelegt (,normiert'), und diese Festlegung gilt primär nur innerhalb des fachlichen Subsystems der Sprache.“272 In Äußerung (c) stellt der Bereich der Wirtschaftspolitik das fachliche Subsystem dar. Der Ausdruck freier Markt kann jedoch auch so betrachtet werden, dass zwar eine spezifische Gesamtbedeutung der feststehenden Wendung existiert, es aber dennoch Unterschiede in der Bedeutung der beiden Komponenten gibt, da das Auftreten der einen Komponente das auftreten der anderen determiniert. Dieser Herleitung folgend ist der Ausdruck in Äußerung (c) als "Nominationsstereotype" zu bewerten:

Es sind Wortverbindungen, deren Gesamtsemantik durch die wendungsexterne Semantik ihrer Komponenten gegeben ist, die sich aber doch noch auf nicht voraussagbare Weise - und sei dies noch so geringfügig - von der einfachen Summe dieser Komponentenbedeutungen unterscheiden. ${ }^{273}$

\section{$\underline{Z u}(\mathrm{~d})$, (f) und (j)}

Die Wendung jemandem (einen) Einblick in etwas verschaffen kann als „Funktionsverbgefüge“ betrachtet werden und gehört der Kategorie der Substantiv-Verb-Kollokationen an; „Funktionsverbgefüge enthalten ein Substantiv (z.B. Entscheidung), das aus einem Verb (entscheiden) nominalisiert wurde, und semantisch ,leere' Verben, mit denen die Aktionsart differenziert werden kann. “274 Dieses Gefüge scheint einen lediglich geringen Grad an Stabilität aufzuweisen; so lässt sich vor allem die auf

\footnotetext{
269 Burger 2002, S. 398

270 DUDEN 1999, S. 1658

271 PONS - Deutsche Idiomatik 1993, S. 306/307

272 Burger, Harald: Phraseologie. Eine Einführung am Beispiel des Deutschen. Berlin 22003, S. 48

273 Fleischer, Wolfgang: Phraseologie der deutschen Gegenwartssprache. Tübingen ${ }^{2} 1997$, S. 58/59

274 Burger 2003, S. 52
} 
demselben Prinzip basierende Wendung jemandem einen Einblick in etwas geben $^{275}$ nachweisen. Funktionsverbgefüge werden auch als "Streckformen “276 bezeichnet und nehmen eine Zwischenstellung zwischen Syntax und Lexik ein. Abschließend sei noch erwähnt, dass mit der Verwendung unterschiedlicher Verben auch der Ausdruck der Aktionsart verändert wird (Einblick verschaffen vs. Einblick geben). Dem Bereich der Funktionsverbgefüge gehört auch die Wendung einen/keinen Anspruch auf etwas haben in Äußerung (j) ebenso an wie der Ausdruck sein Joch abschütteln/abwerfen; dabei ist der letztgenannte Ausdruck als „pathetische, veraltende und seltene" idiomatische Wendung in PONS (1993) ${ }^{277}$ gelistet und findet sich in der Wendung das (schmachvolle) Joch abzuwerfen auch in frühen Parteiprogrammen (KPD 1918/19, S. 107). Im Godesberger Programm lässt sich der Phraseologismus einen Anspruch auf etwas haben in dieser Verwendung insgesamt 7-mal nachweisen.

Der Ausdruck öffentliche Meinung ist dem Bereich der „nominalen Phraseologismen" zuzuordnen; dieser Bereich beinhaltet Phraseologismen, "die die Satzgliedfunktionen Subjekt, Objekt (eventuell auch Attribut) erfüllen können. ${ }^{\prime 278}$ CZERWICK (1996) sieht in dem Ausdruck öffentliche Meinung „ein weiteres funktionales Äquivalent dafür geschaffen, vom Prinzip der Volkssouveränität auf das Repräsentationsprinzip umzusteigen, ohne ersteres formal aufzugeben. ${ }^{279}$ Auf dieses Prinzip wird bei der späteren Betrachtung der Leerformeln erneut eingegangen.

\section{$\underline{\mathrm{Zu}(e)}$}

Der unterstrichene Ausdruck in Äußerung (e) stellt ein Kompositum dar, das eine idiomatisierte Bedeutung aufweist und daher dem Bereich der „Einwortphraseologismen “280 zugerechnet werden kann. Jedoch haben solche Ausdrücke „,in morphosyntaktischer Hinsicht klar den Status von Wörtern und nicht von Phraseologismen. “ ${ }^{281}$ Der Ausdruck in Staatshand steht sinngemäß für die Macht (hier besonders die wirtschaftliche Macht), die in den „Händen des Staates“ liegt, d.h. die Regierung hat

\footnotetext{
275 PONS - Deutsche Idiomatik 1993, S. 150 
durch die verschiedensten Institutionen und durch sonstige Kontrollorgane sowie aufgrund der Gesetzgebung die Verfügungsgewalt inne.

\section{$\underline{\mathrm{Zu}(\mathrm{h}) \text { und (m) }}$}

Die Wendungen jemanden willkommen heißen in (h) und etwas/sich Geltung verschaffen in Äußerung (m) gehören dem Bereich der verbalen Phraseologismen an.

\section{$\underline{\mathrm{Zu}(\mathbf{i})}$}

Bei den Ausdrücken in Äußerung (i) handelt es sich um drei phraseologische Termini zur Bezeichnung einer Partei, eines Landes und einer Regierungsform. Die Wortgruppe freiheitlich-demokratische Grundordnung stellt einen speziellen Fall einer Nominationsstereotype dar. Durch die Terminologisierung einer solchen Wortgruppe wird eine Stabilität des Ausdrucks erzielt und es kann weiterhin zu Wechselbeziehungen zwischen einem solchen politisch-ideologischen Terminus und einem Schlagwort kommen: „Ein derartiger Terminus kann zugleich Schlagwort sein, der Gebrauch als Schlagwort kann andererseits zur Unschärfe des Terminus, zur Entterminologisierung führen. “282

\section{$\underline{\mathrm{Zu}(\mathrm{k}) \text { und (n) }}$}

Die Wendungen zu Unrecht und in Wirklichkeit werden in Äußerung (k) als korrespondierende Form zur Hervorhebung der gegensätzlichen Positionen zwischen den Kommunisten und der SPD gebraucht. Beide Ausdrücke sind strukturelle Phraseologismen und gehören dem Bereich der „präpositionalen und adverbiellen Phraseologismen“ an; sie „erfüllen die syntaktischen Funktionen, die der entsprechenden Wortart zukommen.“283

Ein Beispiel für einen adverbiell verwendeten Phraseologismus findet sich im Godesberger Programm in Äußerung (n); die temporal gebrauchte Wendung auf die Dauer macht dabei eine vage Zeitangabe.

\section{$\underline{\mathrm{Zu}(\mathbf{l})}$}

Die Wendung mehr oder minder in Äußerung (1) ist gleichbedeutend mit dem Ausdruck mehr oder weniger und stellt eine "Paarformel“ oder "Zwillingsformel" dar, die nach folgendem Muster gebildet ist:

Zwei Wörter der gleichen Wortart oder auch zweimal dasselbe Wort werden mit und, einer anderen Konjunktion oder einer Präposition zu einer paarigen Formel verbunden. Wenn zwei verschiedene Wörter

\footnotetext{
282 Fleischer 1997, S. 61
}

283 Burger 2003, S. 43 
vorhanden sind, ist die Reihenfolge entweder völlig festgelegt, oder es besteht zumindest eine Bevorzugung einer Reihenfolge. ${ }^{284}$

\subsubsection{Leerformeln}

„Wer unklar spricht, hat entweder nichts zu sagen oder etwas zu verheimlichen. “285

Die von Kurt Biedenkopf formulierte (polemische) These soll an dieser Stelle als Einleitung des nachfolgenden Untersuchungspunktes dienen. Unter der Bezeichnung Leerformel wird allgemein eine Aussage mit geringem oder keinem Informationsgehalt verstanden. Eine inhaltsarme oder gar inhaltsleere und formelhafte sprachliche Äußerung lässt einerseits einen großen Interpretationsspielraum offen und ermöglicht andererseits das Kaschieren bestimmter Deutungen:

Den Eindruck zu erwecken, man habe sich klar und eindeutig geäuBert, um damit zu kaschieren, dass man systematisch weite Deutungsspielräume und/oder alternative Lesarten eröffnet hat, ist ein Strategietypus, der vor allem verwendet wird, um Adressatengruppen mit divergierenden Präferenzen gleichzeitig zu bedienen, um sich Operationsspielräume offen zu halten und um der eigenen Äußerung im Bedarfsfall eine andere Interpretation als die zunächst nahe gelegte geben zu können. ${ }^{286}$

Leerformeln in Parteiprogrammen resultieren somit aus dem Konflikt zwischen dem Anspruch des Adressaten auf Informationen einerseits, sowie andererseits aus dem Vorsatz der Verfasser, Operationsspielräume offen zu halten und darum keine substanziellen Informationen zu liefern: "Zwar wird zum Thema gesprochen, doch nicht konkret, sondern in Allgemeinplätzen, Selbstverständlichkeiten, Tautologien, Sowohl-alsauch-Bekundungen u.ä. " 287

Diese Tatsache ist der sprachlichen Kreativität und der Lebendigkeit eines Textes allerdings eher nicht zuträglich und auch die Werbefunktion wird nicht zwangsläufig erfüllt, da es durchaus möglich ist, dass „unter den Bedingungen des Marktmodells die typischen Eigenschaften politischer Kommunikation geradezu zwangsläufig dazu führen, daß deren Adressaten, die Bürger, sich davon eher abgestoßen als angezogen fühlen “288:

284 Burger 2003, S. 45

285 CDU (Hrsg.): 22. Bundesparteitag der Christlich Demokratischen Union Deutschlands (18.-20. November 1973). Niederschrift. Hamburg 1973, S. 62

286 Klein 1998, S. 386

287 Klein 1998, S. 383

288 Klein 1996b, S. 7 
Auch noch bei böswilliger Interpretation muß jeder einzelne Satz eines Parteiprogramms unangreifbar sein. Auch noch das aus dem $\mathrm{Zu}$ sammenhang gerissene Zitat daraus darf nur so verstanden werden können, wie es gemeint war. ${ }^{289}$

Insbesondere die letztzitierte Aussage ist eine gesonderte Betrachtung wert. Im Umkehrschluss bedeutet die formulierte These, dass Argumentationen in einem Parteiprogramm aufgrund dessen angestrebter Unverfänglichkeit überhaupt nicht möglich sein können, da die Folgerichtigkeit von Konklusion, Schlusspräsupposition und Argument ein wichtiger Teilaspekt der Argumentation ist. Wenn nun jede Begründung innerhalb einer Argumentation isoliert betrachtet werden kann und dennoch eigenständig in Aussage und Inhalt bleibt, dann ist eine Argumentation nichts anderes als eine Anlagerung von Behauptungen oder allgemein gültiger Aussagen. In extremer Ausprägung bestünde ein Parteiprogramm dann ausschließlich aus Phrasen oder Leerformeln, die nur aneinandergereiht und lediglich nach thematischen Gesichtspunkten untergliedert wären. Solche Aussagen wären in ihrer Formulierung allerdings so vage, dass ihnen kein oder nur geringer präskriptiver Gehalt zuerkannt werden könnte. Die Parteiprogramme werden zu einem späteren Zeitpunkt auf solche Leerformeln hin zu überprüfen sein.

Jedoch gibt es auch Meinungen, die von der Folgerichtigkeit der Aussagen eines Parteiprogramms als unbedingte Voraussetzung überzeugt sind:

Das Programm muß außerdem - auch das ergibt sich aus der Vorstellung rationaler Programmatik - insoweit , aus einem Guß' sein, als die einzelnen Ziele in einem sinnvollen Zusammenhang zueinander stehen, sich zunächst einmal nicht widersprechen, nach Möglichkeit sogar ergänzen. ${ }^{290}$

Eine Leerformel als Aussage verzichtet auf die logische Herleitung und ist daher auch nicht empirisch gehaltvoll. Sie ist allerdings mit einer großen Anzahl anderer Sichtweisen vereinbar oder stimmt mit beliebigen Sachverhalten überein. EROMS (1974) formuliert die zwei nachfolgend zitierten Hauptfunktionen der Verwendung von Leerformeln, die aus verschiedenen Sichtweisen resultieren:

Die politischen Anhänger sollen fühlen, daß einer der ihren spricht oder schreibt. Die Gegner sollen zu der Überzeugung gebracht werden, daß sie die Grundmeinung des Sprechenden teilen, weil die Grundwerte Freiheit, Recht, Mitbestimmung, oder was immer ,aufgerufen' wird, auch die ihren sind, und sie sollen so geneigter gemacht

289 Hermanns 1989, S. 118

290 Flohr 1968, S. 53 
werden, auch den anstehenden politischen Maßnahmen zuzustimmen. ${ }^{291}$

Der Vorteil einer sprachlichen Verwendung von Leerformeln liegt auf der Hand: Die formulierten Aussagen können schwer widerlegt werden und sind daher auch gegen Kritik gewappnet. Für den Wähler ist während der Legislaturperiode der Partei, die er gewählt hat, kaum nachvollziehbar, ob die gesetzten Ziele erreicht wurden, da diese im Programm nicht präzise oder klar dargestellt wurden. Oder deutlicher formuliert: Leerformeln, auf die sich alle einvernehmlich einigen, können die bestehenden Meinungsverschiedenheiten nicht ausgleichen, sondern nur vertuschen. Darüber hinaus wird durch deren Verwendung die Beständigkeit des eigenen Wollens suggeriert. Leerformeln, d.h. inhaltsleere Ausdrücke oder Wortfassaden mehrdeutigen Inhalts, können daher ein sprachliches Mittel zur bewussten Täuschung des Rezipienten sein:

[...] We are in a good position to realize that a situation of ambiguity and confusion is being deliberately fostered by political double-talk and insincerity, with the precise purpose of deceiving the audience. [...] Terminological clarity is a basic requirement for any science, and [...] political scientists in particular have to take stock of the fact that the vocabulary of politics tends to be used for the purpose of beguiling the listener. [...] And contemporary constitutions are, as a rule, bad constitutions - technically speaking. They have come to include unrealistic promises and glamorous professions of faith on the one hand, and numberless frivolous details on the other. [...] That the political scientist, in particular, is responsible for ruling out insincere language, for two reasons: the general reason being that the language of the observer is required to improve (whenever necessary) the language of the observed; while the specific reason is that terminological housecleaning is all the more necessary whenever the political scientist is confronted with terminological distortions that are deliberately fostered with a view to deceiving the audience. ${ }^{292}$

Dieser Darstellung zufolge bedeutet die Verwendung von Leerformeln im politischen Sprachgebrauch, dass solche Aussagen (insbesondere Versprechen) aufgrund der fehlenden logischen Herleitung und des fehlenden Realitätsbezugs unverbindlich sind. Trotzdem sind Leerformeln nicht nur im politischen Sprachgebrauch üblich und nachweisbar:

Inhaltsarme und gar inhaltsleere Formeln können in fast allen Parteiprogrammen nachgewiesen werden. Oft ist zwar in gewissem Um-

291 Eroms, Hans-Werner: „Zur Analyse politischer Sprache.“ In: Linguistik und Didaktik 17. München 1974, S. 5

292 Sartori, Giovanni: „Constitutionalism: A Preliminary Discussion." In: The American Political Science Review (APSR). Volume 56. Menasha, Wisconsin 1962, S. $859 / 862 / 863$ 
fang ersichtlich, was die Verfasser nicht meinen, doch ungewiß, über welche möglichen Sachlagen oder Verhaltensweisen etwas ausgesagt wird. Nicht zufällig bleiben oft gerade die politisch umstrittenen und daher interessanten Grenzfälle unentschieden. ${ }^{293}$

Die Behauptung soll nachfolgend im Hinblick auf das Godesberger Programm und die anderen Parteiprogramme geprüft werden. Dabei sollen die Textstellen und Formulierungen exemplarisch herausgestellt werden, die aufgrund ihrer Abstraktion verschwommen bleiben oder trotz expliziter Nennung keine Aussage beinhalten; die phrasenhaften oder inhaltsarmen Ausdrücke sind unterstrichen.

(43) (a) Im demokratischen Staat muß sich jede Macht öffentlicher Kontrolle fügen. [...] In der vom Gewinn- und Machtstreben bestimmten Wirtschaft und Gesellschaft sind Demokratie, soziale Sicherheit und freie Persönlichkeit gefährdet. Der demokratische Sozialismus erstrebt darum eine neue Wirtschafts- und Sozialordnung. [...] Gemeineigentum ist eine legitime Form der öffentlichen Kontrolle, auf die kein moderner Staat verzichtet. (Godesberger Programm 1959, S. 70/76)

(b) Es ist eine öffentliche Aufgabe die Modernisierung der Landwirtschaft und ihre Leistungsfähigkeit zu fördern. [...] Es ist eine öffentliche Aufgabe, die Krankenhausversorgung sicherzustellen. (Godesberger Programm 1959, S. 77/79)

(c) Das Interesse der Gesamtheit muß über dem Einzelinteresse stehen. (Godesberger Programm 1959, S. 70)

(d) Wettbewerb durch öffentliche Unternehmen ist ein entscheidendes Mittel zur Verhütung privater Marktbeherrschung. Durch solche Unternehmen soll den Interessen der Allgemeinheit Geltung verschafft werden. Sie werden dort zur Notwendigkeit, wo aus natürlichen oder technischen Gründen unerläßliche Leistungen für die Allgemeinheit nur unter Ausschluß eines Wettbewerbs wirtschaftlich vernünftig erbracht werden können. (Godesberger Programm 1959, S. 75)

(e) In seinen Verwaltungsorganen müssen die Interessen der Arbeiter und Angestellten ebenso wie das öffentliche Interesse und das der Verbraucher vertreten sein. Nicht durch zentrale Bürokratie, sondern durch verantwortungsbewußtes Zusammenwirken aller Beteiligten wird der Gemeinschaft am besten gedient. (Godesberger Programm 1959, S. 76)

(f) Gesetzgebung, Regierung und Rechtsprechung sind getrennt voneinander dem Wohle des Ganzen verpflichtet. (Godesberger Programm 1959, S. 72) 
(g) Freiheit und Demokratie in der industriellen Gesellschaft sind nur denkbar, wenn eine ständig wachsende Zahl von Menschen ein gesellschaftliches Bewußtsein entwickelt und zur Mitverantwortung bereit ist. (Godesberger Programm 1959, S. 71)

(h) Die Gliederung der öffentlichen Gewalt in Bund, Ländern und Gemeinden soll die Macht verteilen, die Freiheit stärken und dem Bürger durch Mitbestimmung und Mitverantwortung vielfachen Zugang zu den Institutionen der Demokratie geben. (Godesberger Programm 1959, S. 72)

(i) Die Gesetze müssen der gesellschaftlichen Entwicklung zeitgerecht angeglichen werden, damit sie nicht zum Rechtsbewußtsein in Widerspruch geraten, sondern der Verwirklichung der Rechtsidee dienen. (Godesberger Programm 1959, S. 72)

(j) Wo mit anderen Mitteln eine gesunde Ordnung der wirtschaftlichen Machtverhältnisse nicht gewährleistet werden kann, ist Gemeineigentum zweckmäßig und notwendig. (Godesberger Programm 1959, S. 76)

Bei einer Betrachtung der unterstrichenen Ausdrücke fällt auf, dass diese gar nicht so fremd klingen, sondern vielmehr in aller Munde sind. Allerdings verraten sie nicht, was genau durch deren Verwendung zum Ausdruck kommen soll; sie bleiben in den zitierten Sätzen inhaltsleer und unklar und dennoch jedermann geläufig. Zu einer schnellen und überregionalen Popularisierung von inhaltsarmen oder gar inhaltsleeren Wendungen trägt die mediale Rezeption einen wesentlichen Teil bei. Auffällig ist in ihrer Verwendung, dass sie verhältnismäßig oft im Zusammenhang mit der bestehenden oder einer angestrebten Gesellschaftsordnung gebraucht werden. Die unterstrichenen Ausdrücke wie beispielsweise öffentliche Kontrolle, öffentliche Aufgabe, Interesse der Gesamtheit, öffentliches Interesse oder gesunde Ordnung sind aufgrund der inhaltlichen Vagheit als Leerformeln zu bewerten; als Phraseologismen können die Ausdrücke jedoch ebenso unter dem Aspekt betrachtet werden, dass sie „eine Verbindung von zwei oder mehr Wörtern" darstellen und als „Wortverbindung in der Sprachgemeinschaft, ähnlich wie ein Lexem, als feste Verbindung gebräuchlich" sind.294 Gegen die Betrachtung von Phrasen als Phraseologismen spricht, dass beispielsweise der Ausdruck öffentliches Interesse die jeweiligen Bedeutungen der freien Komponenten behält und sich keine neue Gesamtbedeutung als lexikalische Einheit ergibt. Der Rezipient weiß, dass es hierbei um das Interesse der Öffentlichkeit geht. Die konkrete Bedeutung des Ausdrucks bleibt dennoch unklar und macht die Phrasenhaftigkeit aus, daher wird die Bedeutung des Begriffs Phrase in dieser Untersuchung als imposante Wortfassade verstanden.

294 Burger 2002, S. 392 
Nachfolgend sollen die im Godesberger Programm verwendeten gebräuchlichen und dennoch inhaltlich undeutlichen Ausdrücke ausführlich beleuchtet werden.

\section{$\mathrm{Zu}(\mathbf{a})$}

Der Ausdruck öffentliche Kontrolle bleibt in der Verwendung des Godesberger Programms unklar, obwohl ihm aufgrund der mehrmaligen Erwähnung offensichtlich ein hoher Stellenwert beigemessen wird. Isoliert betrachtet kann mit dem Ausdruck öffentliche Kontrolle ein bestimmter Inhalt assoziiert werden: „Öffentliche Kontrolle im demokratischen Rechtsstaat erfolgt auf vielfältige Art, u.a. durch freie Wahlen, parlamentarische Opposition, freie Presse, Gewaltenteilung und unabhängige Richter. “295 So könnte die öffentliche Kontrolle beispielsweise durch die Polizei als Institution zur Wahrung der öffentlichen Sicherheit (die Sicherheit aller Bürger in der Öffentlichkeit) geschehen, was allerdings nicht im Parteiprogramm genannt wird. Problematisch in Bezug auf das Verständnis wird der Ausdruck im Godesberger Programm durch den Kontext, der dem Ausdruck unmittelbar folgt, denn darin werden die oben im Zitat erwähnten Instanzen nicht genannt. Sie werden zwar an anderer Stelle des Programms explizit genannt, hier jedoch wird der Ausdruck öffentliche Kontrolle vom Rezipienten nicht auf eine solche Instanz bezogen; stattdessen wird öffentliche Kontrolle in einen wirtschaftspolitischen Rahmen eingebunden und kann dadurch auch nicht im Sinne des Zitats verstanden werden. Diese Verständnisschwierigkeit wird zusätzlich dadurch verstärkt, dass der Ausdruck in anderen Kontexten verwendet wird; so stellt sich berechtigt die Frage: Inwiefern ist Gemeineigentum eine legitime Form der öffentlichen Kontrolle? Das Godesberger Programm liefert keine Antwort auf die Frage und lässt diese Aussage in (a) ohne weitere ausführliche Begründung daher als Leerformel stehen.

\section{$\underline{\mathrm{Zu}(\mathrm{b})}$}

Der aus der Publizistik stammende Ausdruck öffentliche Aufgabe bezeichnet allgemein „Phänomene und Prozesse kollektiver Meinungsbildung im öffentlichen Austausch über Themen von öffentlichem Interesse“296; im Zeitungswesen beschreibt man mit dem Ausdruck „Recht u[nd] Pflicht der Presse zur Information, Kontrolle u[nd] Kritik des gesamten öffentlichen Lebens in Staat, Wirtschaft u[nd] Gesellschaft.“" 297 Im Kontext des Godesberger Programms bleibt die Bedeutung jedoch ähnlich verschwommen wie die des Ausdrucks öffentliche Kontrolle in Äußerung (a).

\footnotetext{
295 Weber-Fas 2000, S. 345

296 Jarren/Sarcinelli/Saxer 1998, S. 693

297 Brockhaus/Wahrig 1982, S. 895
} 
Warum ist es eine öffentliche Aufgabe, die Modernisierung der Landwirtschaft und ihre Leistungsfähigkeit zu fördern? Der Ausdruck öffentliche Aufgabe kann als Aufgabe der Öffentlichkeit verstanden werden; somit hat jedermann die Aufgabe, für die Modernisierung und Leistungsfähigkeit der Landwirtschaft zu sorgen. Wie soll dies aber geschehen, da nicht alle Arbeitnehmer in der Agrarwirtschaft tätig sind und die Bürger allgemein nicht in den Wirtschaftszweig der Landwirtschaft eingebunden sind? Es liegt nahe, dass die Unterstützung und Förderung über Steuergelder geschieht; die Landwirtschaft wird dann tatsächlich durch jedermann, genauer durch alle Steuerzahler, gefördert. Dieser Herleitung folgend verbirgt sich hinter Aussage (b) die Forderung der SPD nach Subventionierung der Landwirtschaft: Subventionen sind „öffentliche Finanzhilfen an private Unternehmen oder ganze Wirtschaftszweige besonders zu sozial-, wirtschafts- oder kulturpolitischen Zwecken. Formen der S[ubventionen] sind vor allem unentgeltliche Zuschüsse, zinsgünstige Kredite, Steuervergünstigungen und Bürgschaften. “298 In der zweiten Verwendung des Ausdrucks öffentliche Aufgabe müsste dieses Verständnis auch für die Aufrechterhaltung der Institutionen im Gesundheitswesen gelten. Die Krankenhausversorgung ist demnach Aufgabe der Öffentlichkeit, verkörpert beispielsweise durch den jeweiligen Landkreis und finanziert letztlich durch steuerliche Abgaben. Die abstrakte Verwendung der Leerformel öffentliche Aufgabe entzieht sich der Kritik; dieses sprachliche Handlungsmuster ist weitaus werbewirksamer als das denkbare sprachliche Äquivalent: „Die Krankenhausversorgung ist durch Steuergelder sicherzustellen."

\section{$\underline{\mathrm{Zu}(\mathrm{c}) \text { bis (f) }}$}

Die Aussagen (c) bis (f) stellen prinzipiell geläufige Formeln dar. Die Ausdrücke Interesse der Gesamtheit und Allgemeinheit werden in dieser Untersuchung als Gemeinwohl verstanden, das ,in der politischen Philosophie und in der praktischen Politik den übergreifenden Zweck oder die gemeinsamen Ziele und Werte, auf die hin das Zusammenleben im Staat angelegt ist bzw. angelegt sein soll“ ${ }^{\prime 299}$ bezeichnet. Problematisch ist in den Aussagen allerdings, dass das jeweilige Gemeinwohl nicht definiert ist. ${ }^{300}$ Zwar ist unstrittig, dass in konkreten Situationen Entscheidungen

298 Weber-Fas 2000, S. 497

299 Jarren/Sarcinelli/Saxer 1998, S. 654

300 Dabei ist allerdings anzumerken, dass der Ausdruck Gemeinwohl auf eine lange geschichtliche Tradition zurückblicken kann und auch heutzutage in öffentlichen Debatten oder Diskussion sowie in Parteiprogrammen verwendet wird. Die Aussage, dass das Gemeinwohl oberstes Gesetz sein soll, geht auf die von Marcus Tullius Cicero (106-43 v. Chr.) formulierte Herrschaftsmaxime "Salus publica suprema lex esto" zurück. Weiterhin bestimmt die Bayerische Verfassung in Artikel 3, dass der Bayerische Staat dem Gemeinwohl dient. 
getroffen werden können, die in einer bestimmten Gruppe Einigkeit darüber hervorrufen, dass diese Maßnahmen zu deren Vorteil gereichen oder für die meisten das Beste sind; in den Formulierungen (c) bis (f) werden jedoch keine Gruppen oder Maßnahmen explizit genannt. Die Erkenntnis der Aussage (d), dass beispielsweise niedrige Preise für den Verbraucher dadurch gewährleistet werden, indem öffentliche Unternehmen den für die Preisstabilität notwendigen Wettbewerb sichern und private Marktbeherrschung mit einhergehender Preiswillkür dadurch verhindert wird, ist abhängig vom individuellen Interpretationsvermögen des Rezipienten. Folglich ist die sprachliche Absicht nicht öffentlich ausgewogen wirksam, da sie auf vielfältige Weise rezipiert werden kann. Oder deutlicher formuliert: „Wer etwa einmal versucht hat, praktikable Richtlinien für die Politik öffentlicher Unternehmen, zum Beispiel für ihr Preisgebaren, aufzustellen, der weiß, daß die Floskel ,Interesse der Allgemeinheit' hierbei nicht das Geringste hilft. “" ${ }^{301}$

Weiterhin stellt sich die Frage, worin der Unterschied zwischen einem öffentlichen Interesse, einem Interesse der Gesamtheit und einem Interesse der Allgemeinheit liegt. Hinter der Formel öffentliches Interesse verbirgt sich „ein in verschiedenen gesetzlichen Zusammenhängen verwendeter unbestimmter Rechtsbegriff“, der weiterhin "den Vorrang allgemeiner Belange gegenüber dem individuellen Interesse des Betroffenen“ ${ }^{\prime 302}$ betont. Speziell der zweite Teil des Zitats stimmt mit der Aussage der Äußerung (c) überein. Dennoch erscheint der Ausdruck öffentliches Interesse "für

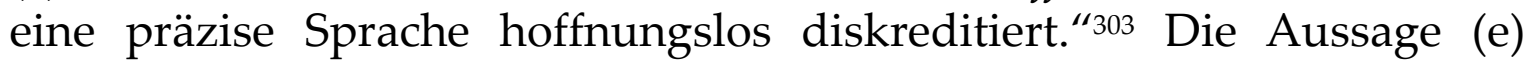
macht durch die explizite Nennung der Arbeiter, Angestellten und Verbraucher als Gesellschafts- bzw. Berufsgruppen zudem deutlich, dass deren Belange nicht in der Formel des öffentlichen Interesses integriert sind, denn sonst wäre deren explizite Erwähnung überflüssig. Hinsichtlich der Äußerung (f) stellt sich die Frage, was die Formel Wohle des Ganzen bedeutet, insbesondere wenn Gesetzgebung, Regierung und Rechtsprechung diesem getrennt voneinander verpflichtet sind. Somit bleibt auch diese Äußerung unklar. Zwar ist es möglich, den Ausdruck Wohle des Ganzen mit dem Ausdruck Gemeinwohl als „das öffentliche Interesse der Gesamtheit im Unterschied zum Privatinteresse einer Person“304 gleichzusetzen; trotz Erwähnung in der Verfassung (,Wohle der Allgemeinheit") sind beide Begriffe unbestimmt und bedürfen im Einzelfall näherer Konkretisierung. CZERWICK (1996) betrachtet die Ausdrücke Allgemeinwohl und öffentliches Interesse stellvertretend für Volkssouveränität, da es nahe liegt,

\footnotetext{
$301 \quad$ Flohr 1968, S. 73

302 Weber-Fas 2000, S. 346

303 Flohr 1968, S. 74

304 Weber-Fas 2000, S. 153
} 
daß in der Politik auf den Begriff der Volkssouveränität, selbst in seiner durch das Repräsentationsprinzip eingeschränkten Bedeutung, verzichtet und er durch Begriffe wie ,Allgemeinwohl' oder ,öffentliches Interesse' ersetzt wird. Damit wird ein scheinbar neutraler Bezugspunkt geschaffen, der es als gerechtfertigt erscheinen läßt, unter gewissen Umständen auch gegen das Volk eine Politik für das Volk zu machen. ${ }^{305}$

\section{$\underline{\mathrm{Zu}}(\mathrm{g})$}

Die in (g) getroffene Aussage kann nicht widerlegt werden. Es ist wahrscheinlich tatsächlich so, dass Freiheit und Demokratie an Mitverantwortung und gesellschaftliches Bewußtsein gebunden sind. Die Aussage kann nicht widerlegt werden, weil keine Anknüpfungsmöglichkeiten für Kritik gegeben sind. Ob der Ausschließlichkeitsanspruch durch nur gerechtfertigt ist, kann an dieser Stelle ebenfalls nicht beantwortet, sondern lediglich in Frage gestellt werden. Wie kann nachvollzogen oder geprüft werden, ob jemand ein gesellschaftliches Bewusstsein besitzt oder entwickelt hat?

Die Fragwürdigkeit der Rede vom gesellschaftlichen Bewußtsein ergibt sich nicht nur daraus, daß mit dem Wort ,Bewußtsein' ideologisch Schindluder getrieben worden ist und noch wird, sondern auch aus der Beifügung ,gesellschaftlich', dieser blassen und deshalb beliebten Vokabel. ${ }^{306}$

Dem Rezipienten erschließt sich die Bedeutung der Leerformel des gesellschaftlichen Bewusstseins nur über die Parteigeschichte der SPD:

Nur wer die Tradition des sozialistischen Denkens kennt, kann mit dieser Formel etwas anfangen. Das genügt nicht für Programme, die das ganze Volk soll verstehen können. ${ }^{307}$

\section{$\underline{\mathrm{Zu}(\mathrm{h})}$}

Hinsichtlich der Aussage (h) müsste die Phrase des vielfachen Zugangs zu den Institutionen der Demokratie präzisiert werden. Schließlich soll dadurch der SPD zufolge die Freiheit gestärkt sowie Mitbestimmung und Mitverantwortung ermöglicht werden. Was bedeutet vielfacher Zugang? Diese erste Frage mündet zwangsläufig in die nächste, nämlich welche Institutionen in der Bundesrepublik Deutschland Institutionen der Demokratie sind. Diese werden im Godesberger Programm nicht erwähnt, wahrscheinlich sind damit aber Gerichte oder auch der Bundestag gemeint. Da die Ausdrücke Mitbestimmung und Mitverantwortung genannt

\footnotetext{
305 Czerwick 1996, S. 59

306 Flohr 1968, S. 73

307 Flohr 1968, S. 73
} 
wurden, kann die Äußerung (h) auch als korrespondierende Aussage mit dem Bitt- und Beschwerderecht (Petitionsrecht) des Grundgesetztextes verstanden werden (die Äußerung (h) müsste dann im oben behandelten Kapitel Das Godesberger Programm als Grundgesetztext aufgelistet sein). Im Grundgesetz heißt es:

Jedermann hat das Recht, sich einzeln oder in Gemeinschaft mit anderen schriftlich mit Bitten oder Beschwerden an die zuständigen Stellen und an die Volksvertretung zu wenden. (Art. 17)

\section{$\underline{\mathrm{Zu}(\mathbf{i})}$}

Wenn Gesetze der gesellschaftlichen Entwicklung zeitgerecht angeglichen werden, bedeutet diese Tatsache dann, dass auch bestimmte Straftaten angeglichen werden? Leider wird im Godesberger Programm kein Beispiel für an die Zeit angepasste Gesetze angeführt. Die Erwähnung erweckt allerdings den Eindruck, dass die SPD nicht altmodisch oder gar rückwärtsgewandt erscheint, sondern sich vielmehr als moderne Partei darstellt, die auf zeitgenössische, d.h. aktuelle gesellschaftliche Fragen, eingeht und die bestehenden Gesetze daran ausrichtet. Wie die konkrete zeitgerechte Angleichung eines Gesetzes aussieht bleibt ebenso unklar wie die Leerformel der Verwirklichung der Rechtsidee.

\section{$\underline{Z u(j)}$}

Die Äußerung (j) findet sich im Godesberger Programm unter dem Kapitel Eigentum und Macht. Aufgrund der Nennung der Ausdrücke Gemeineigentum und wirtschaftliche Machtverhältnisse geht es in Äußerung (j) möglicherweise um die Verteilung des Eigentums und der wirtschaftlichen Macht; daher kann gesund im Sinne von ausgewogen oder gerecht verteilt verstanden werden. Dennoch bleibt der Ausdruck gesunde Ordnung als Leerformel bestehen, weil er ohne weiterführende Erläuterung nichts besagt.

Bei der Betrachtung des Godesberger Programms im Hinblick auf die Verwendung von Phraseologismen oder Leerformeln fällt auf, dass erste kaum nachweisbar sind, Leerformeln jedoch häufig verwendet werden. Diese Feststellung liegt in der Konzeption des Grundsatzprogramms begründet; die Formulierungen müssen allgemein genug gehalten sein um ausreichenden Auslegungsspielraum für das darauf basierende Wahlprogramm zu gewährleisten. Als Erklärung mag weiterhin folgende Behauptung gelten:

Diese Abstraktheit und Unpersönlichkeit hängt natürlich mit der weitgehenden Indirektheit der Institutionalisierung des öffentlichen Handelns in unserer modernen arbeitsteiligen Massengesellschaft zu- 
sammen, ist aber genau in diesem Zusammenhang auch zugleich eine Erscheinung des sprachlichen Ausdrucksstils. ${ }^{308}$

Die im Zitat erwähnte Abstraktheit und Unpersönlichkeit wird weiterhin dadurch hervorgerufen, dass die Verwendung von Leerformeln oder Abstraktwörter die Bezeichnung von Personen ersetzt. So finden sich im Godesberger Programm folgende metonymisch übertragene Handlungs-, Vorgangs- oder Zustandsbezeichnungen, die stellvertretend für bestimmte Adressatengruppen stehen: Arbeits- und Sozialgesetzgebung (1) sowie Gesetzgebung (1) für "Gesetzgeber" oder Rechtsprechung (3) für "Richter". Zwar wird der Ausdruck Richter im Godesberger Programm auch explizit genannt. Die Verwendung solcher Abstraktwörter ist aber eine Möglichkeit zu vermeiden, dass handelnde Einzelpersonen oder Mehrheiten, die ein bestimmtes Machtpotential haben, genannt werden müssen.

Weiterhin liegt der Aussage des oben angeführten Zitats folgend die Vermutung nahe, dass die Verwendung von Leerformeln mit der Entwicklung der Massenmedien sowie mit der Entwicklung des bereits beschriebenen Marktmodells einhergeht. Diese Vermutung ist bedingt berechtigt, da insbesondere das Fernsehen die Politik und deren Wahrnehmung verändert hat:

Der Spielraum für politische Kommunikation wird festgelegt erstens durch die Geltung einer Anzahl Kommunikationsnormen, zweitens durch die Medienbedingungen. Kommunikationsnormen und Medienbedingungen bestimmen die Art, wie Politiker reden, wie sie mit Themen umgehen, wie sie sich den Bürgern gegenüber geben. ${ }^{309}$

Allerdings lassen sich Leerformeln bereits in sehr frühen Parteiprogrammen nachweisen, als die heutige massenmediale Informationsstruktur noch nicht existierte: „Eigentum soll uns Verpflichtung zum Dienst am Ganzen sein“ (Konservative Volkspartei 1930, S. 545). Äußerungen zur Frage des Gewinnstrebens und der Vermögensbildung sowie die Frage, welche Gewinne gerechtfertigt und welche ungerechtfertigt sind, werden offensichtlich in nahezu allen Parteiprogrammen bis in die Gegenwart thematisiert; die von der jeweiligen Partei geforderten Beschränkungen in Äußerung (b), (c) und (d) bleiben jedoch wie im Godesberger Programm unklar. Äußerung (e) kann zwar dahingehend interpretiert werden, dass sich die Partei gegen eine aggressive Außenpolitik ausspricht, die konkreten außenpolitischen Ansichten werden jedoch von den allgemeingültigen Abstrakta überlagert und bleiben zwar nicht inhaltsleer, aber inhaltsarm und keineswegs eindeutig:

308 v. Polenz 1985, S. 36

309 Klein 1996b, S. 7/8 
(44) (a) In Erwägung, daß die politische und ökonomische Befreiung der Arbeiterklasse nur möglich ist, wenn diese gemeinsam und einheitlich den Kampf führt, gibt sich die sozialdemokratische Arbeiterpartei eine einheitliche Organisation, welche es aber auch jedem einzelnen ermöglicht, seinen Einfluß für das Wohl der Gesamtheit geltend zu machen. (Eisenacher Programm 1869, S. 312)

(b) Die Deutsche Volkspartei fordert für jeden Staatsbürger das Recht der freien Entfaltung seiner Kräfte. Das Streben nach Gewinn muß aber bei dem einzelnen seine sittlichen Schranken finden in der Rücksichtnahme auf das Wohl und die Bedürfnisse der übrigen Volksgenossen. (DVP 1919, S. 525)

(c) Jede lebensfähige Volkswirtschaft baut sich auf dem Privateigentum und der Eigenwirtschaft auf. Unternehmensgeist und Erwerbssinn des einzelnen sind die Grundlage unserer wirtschaftlichen Arbeit. Wir verlangen, daß sie sich in den Grenzen des Gemeinwohles halten [...]. (DNVP 1920, S. 540)

(d) Soziale Klassensonderung von oben und unten wollen wir überwinden. Das notwendige Streben des einzelnen nach wirtschaftlichem Erfolge darf Nation und Staat nicht schwächen. Eigentum soll uns Verpflichtung zum Dienst am Ganzen sein. (Konservative Volkspartei 1930, S. 545)

(e) Auch die außenpolitischen Richtlinien sind für eine Partei der Mitte mit den Begriffen Gleichberechtigung, Freiheit, Gerechtigkeit, Friede und Verständigung eindeutig vorgezeichnet. (Zentrum 1951, S. 723)

Idiome und Sprichwörter fallen zwar in den Bereich der Phraseologie, sie sind allerdings im Godesberger Programm und in den anderen Parteiprogrammen aufgrund mangelnden Öffentlichkeitsbezugs ebenso selten nachweisbar wie Parolen (singuläre Verwendungen werden in einem nachfolgenden Kapitel jeweils zitiert); sie sind vielmehr typisch für die politische Sprachverwendung in Reden, Presseerklärungen oder Debatten:

In der Markiertheit der bildhaften Phraseologismen, Sprichwörter und Zitate sowie ihrer außerordentlichen Öffentlichkeitswirksamkeit ist ein wichtiger Grund dafür zu sehen, daß etwa verbale Idiome im Sprachgebrauch deutscher Politiker zugenommen haben. Infolge der zunehmenden Öffentlichkeitswirksamkeit parlamentarischen Sprechens, der immer bedeutender werdenden Rolle der Presse und schließlich des Erfordernisses, in immer kürzeren Hörfunk- und TVAusschnitten - oft sind es nur wenige Sekunden - Botschaften zu vermitteln, haben Politiker das Sprechen in Bildern und prägnanten Formeln entwickelt. 310

310 Elspaß 2000, S. 282 
Die im Zitat dargestellten Rahmenbedingungen treffen auf die Verwendung von Leerformeln nicht zu. Vielmehr schlägt sich die Erwähnung von solchen Leerformeln im ästhetischen Empfinden des Rezipienten negativ nieder und die Werbefunktion als eine der wichtigsten Funktionen des Parteiprogramms wird nicht erfüllt:

Es läuft auf ein Paradox hinaus: Obwohl politische Kommunikation darauf ausgerichtet ist, zu überzeugen und für sich einzunehmen, sind es deren Strukturmerkmale selbst, die Politikverdrossenheit oder besser gesagt: Parteienverdrossenheit begünstigen.

Es ist daher durchaus denkbar, dass Teile der Wählerschaft in einem Grundsatzprogramm aufgrund der schemenhaften Abhandlung der phrasenhaften Formulierungen und Leerformeln den Charakter eines „Warenhauskatalog[s]“"311 erkennen.

Da hinsichtlich der Erwähnung des Katalogstils eine stilistische Erscheinungsform von Parteiprogrammen genannt wurde und das nachfolgende Kapitel 2.2 stilistische Merkmale der Wortverwendungen aufgreift, bieten sich eine ausführliche Darlegung des Stilbegriffs und mögliche Definitionen an dieser Stelle an.

\subsubsection{Aspekte der stilistischen Beschreibung}

Wer von einem Parteiprogramm schöngeistige Stilübungen und tiefschürfende philosophische Erörterungen erwartet oder gar verlangt, überfordert es. ${ }^{312}$

Oder noch deutlicher formuliert:

Alles Spielerische, Ambivalente, Problematische muß vermieden werden in einem Text, der unter solchen Bedingungen entsteht, alles Gewagte, Outrierte, Pointierte, denn es könnte mißverstanden werden; damit auch alles Witzige. ${ }^{313}$

Die oben zitierte Aussage soll in der folgenden Untersuchung des Stils des Godesberger Programms mitberücksichtigt werden; unter Berücksichtigung aller bisher in dieser Analyse zitierten Äußerungen und der daraus resultierenden Erkenntnisse scheint es geboten, bei der Analyse des Stil stilistisch auffällige Textpassagen des Godesberger Programms mit dem übrigen Text zu vergleichen und dadurch signifikante Abweichungen zu verdeutlichen. Unter Stil wird primär die stilistische Ausprägung eines Textes verstanden. Dies ist insofern problematisch, als der Begriff des Stils kaum zu verallgemeinern oder zu definieren ist. Es sollen nachfol-

\footnotetext{
311 Klein 1996a, S. 203

312 Schönbohm 1974, S. 17

313 Hermanns 1989, S. 118
} 
gend einige Theorien und Definitionen wiedergegeben werden, um mit gefestigterem Wissen die stilistischen Ausprägungen nachvollziehen zu können.

Stil ist ein „äußerst komplexes Phänomen, das von vielen verschiedenen Standpunkten aus betrachtet werden kann"314 und unter dessen Begriff in diesem Zusammenhang die Machart eines Textes verstanden wird. So hat prinzipiell jede mündliche oder schriftliche sprachliche Äußerung einen speziellen Stil, der vom Adressatenkreis, von bestimmten Situationen der Sprachverwendung oder beabsichtigten Wirkungen definiert wird; es geht folglich um die relevante Art der sprachlichen Handlungsdurchführung.

„Eine Sprachhandlungstheorie ist für die Beschreibung von Stil angemessen, da die Beteiligten Stil in verschiedenen Sprachhandlungsperspektiven Relevanz beimessen. " ${ }^{15}$ Danach ist auch die oben geleistete Betrachtung und Analyse der Sprechakte sowie der Exkurs in die Sprachhandlungstheorie als Teil des für ein Grundsatzprogramm charakteristischen Stils gerechtfertigt.

Stil ist allerdings nicht nur als bloße Form und Struktur zu verstehen, sondern ebenso als sinnhafte Form und bedeutsame Struktur; Stil geht folglich mit einer Funktion einher. Im Hinblick auf die Textgattung des Parteiprogramms als Gebrauchs- oder Anleitungstext ist der Stil daher als Gebrauchsstil oder als situationsgerechter funktionaler Stil zu verstehen und damit Gegenstand der Funktionalstilistik:

Die Funktionalstilistik beschäftigt sich [...] mit der gegenwärtigen Existenzform dieser Varianten des Sprachgebrauchs als funktional differenzierter, gesellschaftlich verbindlicher Muster der Sprachverwendung in konkreten kommunikativen Situationen. ${ }^{316}$

Die im vorangegangenen Kapitel behandelten Kategorien der Lexik und Semantik sind als verwendetes Vokabular ebenso Bestandteil des Stils wie die Grammatik als stabile linguistische Größe; allerdings ist die Grammatik, verglichen mit der Semantik, als die stabilere Größe anzusehen, da Bedeutungen von Wörtern in stärkerem Maße vom jeweiligen Kontext abhängen. So können beispielsweise fachwissenschaftliche Texte unterschiedlicher thematischer Gebiete durchaus im gleichen Stil verfasst sein, obwohl sich die verwendeten spezifischen Termini völlig voneinander unterscheiden. Außerdem kann die Bedeutung von Wörtern in verschiedenen Sprachräumen unterschiedlich sein. Die lexikalische und

314 Spencer, John (Hrsg.): Linguistik und Stil. Heidelberg 1972, S. 5

315 Sandig 1986, S. 32

316 Gläser, Rosemarie: „Die Kategorie ,Funktionalstil' in soziolinguistischer Sicht." In: Zeitschrift für Phonetik, Sprachwissenschaft und Kommunikationsforschung (Band 27). Berlin 1974, S. 487 
morphologische Stiluntersuchung muss daher um die Analyse der grammatikalischen Erscheinungen (Syntax) erweitert werden.

Wird Grammatik als "das natürliche gewachsene und [...] für eine Sprachgemeinschaft gültige Regelsystem “317, das von allen Sprechern einer Sprache in der Kindheit erworben und als kollektiver Besitz der Sprachgemeinschaft von Generation zu Generation weitergegeben wird verstanden, dann ist dieser Ausdruck als komplexes System mit autonomer Gültigkeit zu verstehen. Nach den Regeln der Grammatik werden Wörter gebildet, zu Phrasen oder Sätzen gefügt und Sätze mit anderen $\mathrm{zu}$ Abschnitten und Texten verbunden. Unter grammatikalischem Gesichtspunkt betrachtet besitzt jeder Text demnach Stil, solange die Variationsmöglichkeiten der sprachlichen Handlung von der Grammatik akzeptiert werden. Der Begriff des Stils muss jedoch nicht notwendigerweise die Ästhetik als Nebenbedeutung beinhalten. Allerdings wird Stil häufig mit sprachlicher Kreativität gleichgesetzt, d.h. als individuelle und kreative Verwertung des Sprachmaterials durch den Verfasser eines Textes. Ein Text ohne Variation in der sprachlichen Handlung hat jedoch trotzdem einen anderen Stil, eine andere Machart, als einer, der alle gegebenen Möglichkeiten der Sprachgestaltung ausschöpft, den Rahmen der Grammatikalität jedoch nicht überschreitet.

„Allen Stilbegriffen liegt ein Bewußtsein von Normen zugrunde mit der Möglichkeit, von diesen Normen abzuweichen. “318 Folglich kann Stil nach dieser Definition als Abweichung von der grammatikalischen Norm verstanden werden; "Stil hat ein Text dann, wenn er sich nicht ausschließlich aus obligatorischen grammatischen Regeln bzw. verbindlichen Gesetzmäßigkeiten für die Wort- Satzbildung und des semantischen Systems herleiten läßt." ${ }^{\prime 319}$ Bei einer solchen Abweichungsstilistik bleibt der Stilcharakter allerdings den lediglich außergewöhnlichen Sprachgestaltungen vorbehalten. Erst durch das Aufstellen einer verbindlichen Norm können bestimmte Sprachgestaltungen als außergewöhnlich gelten und als mögliche Abweichungen benannt werden,

und so ist es wahrscheinlich ratsam, als Norm, an der das Individuelle eines bestimmten Textes gemessen wird, nicht die Sprache als ganzes zu nehmen, sondern nur jenen Teil der Sprache, der in einem besonders relevanten Verhältnis zu dem zu analysierenden Text steht. ${ }^{320}$

317 Volmert 1999, S. 13

318 Spencer/Gregory 1972, S. 87

319 Marx-Nordin, Signe: Studien zum Stil deutschsprachiger Parteiprogramme. Ein Beitrag zu einer syntaxorientierten Stilistik. Göteborg 1979, S. 17

320 Enkvist 1972, S. 23 
Allerdings sind „weder der Begriff der sprachlichen Norm noch der der Abweichung davon klar festlegbar [...].“321 Aus diesen Beschreibungen ergibt sich somit die folgende Definition des Ausdrucks Stil:

Der Stil eines Textes ist eine Funktion des Aggregates der Verhältnisse zwischen den Häufigkeitswerten der phonologischen, grammatischen und lexikalischen Größen und den Häufigkeitswerten der entsprechenden Größen in einer kontextverwandten Norm. ${ }^{322}$

Unter dem Ausdruck des Aggregats ist somit ein mehrgliedriges Ganzes $\mathrm{zu}$ verstehen, das sich als Anhäufungen aus den stilistischen Eigenheiten einerseits und möglichen adäquaten Formen der Norm andererseits zusammensetzt. Die in der oben zitierten Definition erwähnten „phonologischen Größen“ können in dieser Analyse nicht berücksichtigt werden, der Schwerpunkt wird in der Untersuchung vielmehr auf den "grammatischen und lexikalischen Größen" liegen.

Unter der Annahme, dass „ein Stil [...] - implizit - immer ein von anderen Stilen verschiedener Stil eines Textes “ ${ }^{\prime 323}$ ist, dann setzt eine Untersuchung des Stils eines Textes sowohl die Beschreibung der charakteristischen Eigenschaften als auch den Vergleich mit einer möglichen alternativen Formulierung und der eventuell daraus resultierenden Bedeutungsdifferenz voraus. So lässt sich beispielsweise feststellen, dass die Sätze „Ein Mann kam plötzlich aus dem Haus“, „Plötzlich kam ein Mann aus dem Hause“, "Aus dem Hause kam plötzlich ein Mann“ sowie „Kam da plötzlich ein Mann aus dem Hause“324 allesamt sowohl grammatikalisch, als auch akzeptabel sind und den gleichen Sachverhalt darstellen, wenn auch auf verschiedene Art und Weise:

All das sind mögliche Wortstellungen. Es ist nicht so, daß eine die übliche ist und die andern als Abweichungen auffällig würden. Welche Fügung im Augenblick des Sprechens gewählt wird, hängt von den Umständen dieses Augenblicks, der Situation, der Hörerschaft, dem Zusammenhang u.s.f. ab. Allgemein könne wir sagen: hängt von der Perspektive ab, in der der Sachverhalt sprachlich dargestellt wird. ${ }^{325}$

Dennoch unterscheiden sich die Sätze hinsichtlich der Wahrscheinlichkeit ihres Auftretens einerseits sowie der unterschiedlichen Akzentuierung aufgrund der veränderten Satzstellung andererseits. Die Wahrscheinlichkeit und Häufigkeit von bestimmten Formen, d.h. die

321 Sowinski, Bernhard: Deutsche Stilistik. Beobachtungen zur Sprachverwendung und Sprachgestaltung im Deutschen. Frankfurt 1973, S. 19

322 Enkvist 1972, S. 26

323 Sandig 1986, S. 18

324 Kayser, Wolfgang: Das sprachliche Kunstwerk. Eine Einführung in die Literaturwissenschaft. Tübingen/Basel ${ }^{201992, ~ S . ~} 129$

325 Kayser 1992, S. 129 
signifikante Anhäufung von bestimmten Merkmalen, kann ebenso ein Mittel der Hervorhebung sein wie die singuläre Variation, die als selten vorkommende Konstruktion eine auffallende Form darstellt und dadurch einen Akzent zu geben in der Lage ist. Dieser Herleitung folgend ist der Stil einer sprachlichen Handlung determiniert als realisierte Wahl zwischen fakultativen Möglichkeiten. Die Häufigkeit und Wahrscheinlichkeit bestimmter Äußerungsformen fungieren somit als Stildeterminanten.

Den Ausführungen folgend wird der Begriff des Stils eines bestimmten Dokumentes durch die Merkmale definiert, die dieses Dokument objektiv kennzeichnen; indem die Merkmale eines bestimmten Textes deskriptiv dargelegt werden wird auch die folgende Analyse weitestgehend auf diesem Verständnis des Stilbegriffs fußen.

Die Methode der Erschließung der relevanten Merkmale des jeweils gewählten sprachlichen Gebildes definiert den Stilbegriff weiterhin. Es hängt allerdings maßgeblich von der begrifflichen Festlegung des Stilbegriffs ab, welche sprachlichen Äußerungen danach als stilistisch relevant angesehen werden und welche nicht. Weiterhin ist die Wahrnehmung eines bestimmten Stils abhängig von der individuellen linguistischen Erfahrung des Rezipienten und damit nicht einheitlich. Bei der Analyse eines Stils werden diese Erfahrungen in Erwartungen umgewandelt, die der jeweilige Text entweder erfüllt oder nicht; folglich wechseln in einer Stilanalyse "früher konstatierte kontextbedingte Häufigkeitswerte zu momentanen kontextbedingten Erwartungen, an deren Aggregat der Text gemessen wird.“326

\subsection{Aspekte der sprachlichen Gestalt}

\subsubsection{Lexik/Semantik: Wortschatz-Charakteristik}

Wenn es in den folgenden Kapiteln um die Aspekte der sprachlichen Gestalt geht werden damit primär die linguistischen Disziplinen der Lexikologie und der Semantik angesprochen. Dabei sieht die Lexikologie ihren sprachwissenschaftlichen Gegenstand

im Inventar lexikalischer Zeichen (Morphemen, Wörtern und festen Wortgruppen), im Aufbau des Wortschatzes und im Regelsystem, das Wortgebrauch und -verstehen bestimmt. Sie untersucht und beschreibt den Wortbestand einer Sprache, seine Schichtung und Struk-

326 Enkvist 1972, S. 26 
tur, Bildung, Bedeutung und Funktionen seiner Elemente. Sie ist die Theorie des lexikalischen Teilsystems, des Lexikons. ${ }^{327}$

Es geht bei der Lexikologie folglich um die umfassende Lehre vom Wort, d.h. die Theorie der Wortform sowie die grammatischen Eigenschaften. LINKE/NUSSBAUMER/PORTMANN (1996) grenzen die Lexikologie als „lexikalische Semantik“ daher von der Semantik ab, bei der man sich "ganz allgemein mit der Bedeutung von (sprachlichen) Zeichen “328 beschäftigt.

\subsubsection{Grundlegende Annahmen zur Wortschatzbetrachtung}

In den folgenden Kapiteln soll nun der in den Parteiprogrammen verwendete politische Wortschatz herausgearbeitet werden, dessen Bedeutung für das (sprachliche) Handeln der Parteien oder eines Politikers allgemein eminent ist:

Für die Sprachverwendung in der Politik ist das Lexikon zwar nicht das einzige, aber das wichtigste Instrument sprachlichen Handelns. Der Politiker als handelnder Akteur steht täglich vor vielfältigen Selektionsentscheidungen, die sich für ihn in der Frage bündeln: Wie benenne ich was wie für wen in welcher Kommunikationssituation?329

Bei aller Anschaulichkeit der oben zitierten Formulierung muss aber festgehalten werden, dass nicht das Lexikon benutzt wird, sondern die Elemente des Lexikons, da das Lexikon als Bezeichnung für das „strukturierte Inventar der Lexeme“330 verwendet wird; die Lexeme oder Elemente wiederum sind „Benennungseinheiten, Wörter und feste Wortverbindungen, die als relativ feste Zuordnungen von Formativ und Bedeutung reproduzierbar sind, gespeichert werden und Basiselemente für die Bildung von Sätzen und Texten [... $]^{\prime 331}$ darstellen.

An dieser Stelle soll noch eine Anmerkung zum politischen Lexikon und dem Nominationskonzept, d.h. der Nominationstheorie als Sprachverwendungslehre gemacht werden. Zwar sind die Bereiche des Lexikons einerseits und der Sprachverwendung andererseits zu differenzieren, man kann jedoch auch von einem Verhältnis gegenseitiger Konstitution bzw. Modifikation ausgehen

zwischen dem lexikalischen Inventar einerseits (,Wortschatz') und andererseits der kommunikativen Nutzung dieses Inventars durch die Sprecher (,Wortgebrauch'). Diese Unterscheidung von Sprachin-

327 Schippan, Thea: Lexikologie der deutschen Gegenwartssprache. Tübingen 22002, S. 1

328 Linke/Nussbaumer/Portmann 1996, S. 132

329 Girnth 2002, S. 47

330 Schippan 2002, S. 1

331 Schippan 2002, S. 1 
ventar und Sprachhandlung, die im folgenden als Lexikon und Nomination terminologisiert werden soll, ist gerade dann notwendig, wenn beide Bereiche als interdependent in dem Sinne angesehen werden, daß jede kommunikative Nutzung das Inventar sowohl voraussetzt als auch potentiell modifiziert. ${ }^{332}$

Ausgangspunkt der nachfolgenden Untersuchungsschwerpunkte ist die von GLONING (2003) formulierte Grundfrage hinsichtlich einer historischen Wortschatzanalyse:

Über welche sprachlichen Mittel mußte ein Sprecher in einem bestimmten Sprachstadium verfügen, wenn er eine bestimmte sprachliche Handlung machen und dabei bestimmte kommunikative Funktionen realisieren wollte?333

Ein wesentlicher Aspekt der Wortschatzforschung geht mit dem Ziel einher, den Wortschatz der sprachlichen Handlungen einer zeitgenössischen Sprechergruppe $\mathrm{zu}$ beschreiben, $\mathrm{zu}$ ordnen und $\mathrm{zu}$ analysieren. Dabei ist von der grundlegenden Annahme auszugehen, dass Wortschätze auf komplexe Weise organisiert sind und dass Wortschätze dynamisch sind ${ }^{334}$; so resultiert diese Annahme aus den Auffassungen,

daß Wortschätze als organisierte Gebilde zu betrachten sind, daß also zwischen den einzelnen Wortschatzelementen vielfältige Zusammenhänge bestehen, die es zu erhellen und zu beschreiben gilt. Zum anderen entwickeln sich Wortschätze mit ihren Elementen und mit den Zusammenhängen im Wortschatz laufend in der Zeit. ${ }^{335}$

Dieser Aussage entsprechend handelt es sich bei dem Wortschatz einer Sprechergemeinschaft nicht um eine willkürliche und zusammenhanglose Ansammlung von Wörtern, sondern vielmehr um ein strukturiertes Gefüge, obgleich dessen Organisation selten offensichtlich ist. Weiterhin kann man vermuten, dass die einzelnen Wortschatzelemente einem Geflecht ähnlich systematisch miteinander verknüpft sind. Die Annahme des Wortschatzes als Beziehungsgeflecht erinnert an die Definition eines Textes:

Andererseits untersucht die Textlinguistik den Bau und die Struktur von Texten, d.h. sie geht der Frage nach, welche sprachlichen Bauelemente Texte konstituieren, wie die einzelnen Elemente (z.B. Sätze,

332 Herrgen 2000, S. 134

333 Gloning, Thomas: Organisation und Entwicklung historischer Wortschätze. Lexikologische Konzeption und exemplarische Untersuchungen zum deutschen Wortschatz um 1600. Tübingen 2003, S. 39

334 Gloning 2003, S. 1ff.

335 Gloning 2003, S. 23 
Textabschnitte) systematisch zusammenhängen und wie sie zu Texten verbunden werden. 336

Es muss dabei auch berücksichtigt werden, dass die einzelnen Elemente eines Wortschatzes in einer dynamischen Beziehung stehen und dadurch den Aspekt des Wachstums, d.h. der Wortschatzentwicklung, veranschaulichen. Hinsichtlich dieses Aspekts lässt sich daher vermuten, dass sich durch die Wortverwendungen, z.B. bei den thematischen Schwerpunkten, das Zeitgeschehen widerspiegelt und die jeweils vorherrschenden gesellschaftlichen Rahmenbedingungen zum Ausdruck kommen; danach kann der Wortschatz als Ausdruck der sprachlichen Handlungen einer Sprechergemeinschaft gewertet werden.

Der erwähnte Bau eines Textes legt den Vergleich des Wortschatzes mit einem Gebäude nahe. Dabei sind die Architektur des Gebäudes von Interesse, insbesondere die Raumaufteilung und die Raumverknüpfungen (dynamische Struktur), sowie der Umbau, die Abrissarbeiten und die Neubauten (Wortschatzentwicklung). Entsprechend wird die Gesamtheit der sprachlichen Mittel einer Sprachgemeinschaft als kommunikativer Haushalt bezeichnet.

Wortschätze bzw. Wortschatzelemente können regional verschieden sein; identische Dinge oder Sachverhalte können somit auf unterschiedliche Weise bezeichnet werden, allerdings ist das Kriterium der Regionalität ein Mittel, Wortschätze voneinander abzugrenzen und dient weniger der Strukturierung. In den untersuchten Parteiprogrammen finden sich die Ausdrücke Arbeitnehmer als Element des Wortschatzes in der Bundesrepublik Deutschland und Werktätiger als Element des Wortschatzes der ehemaligen DDR. Beide Ausdrücke bezeichnen einen Arbeiter oder Angestellten als eine Person, die in einem Arbeitsverhältnis steht und sind daher referenzidentisch. Eine Untersuchung der Parteiprogramme nach diesem Kriterium dient jedoch lediglich einer Beschreibung regionaler Ausprägungen und wird in dieser Analyse nicht durchgeführt. Zweckdienlicher ist die Untersuchung der Wortschatzelemente nach ideologischen Ausprägungen.

Wie oben bereits angesprochen spiegelt die Sprachverwendung die ideologische Färbung der jeweiligen Partei wieder. Eines der Ziele der Wortschatzbetrachtung ist es daher, durch die Herausstellung prägnanter Ausdrücke und Begriffe den Einfluss der Ideologie der jeweiligen Partei auf die Sprachverwendung in deren Parteiprogrammen exemplarisch darzulegen. Somit fungiert der Wortschatz als Indikator bei der Fragestellung, wie sich die parteispezifischen ideologischen Färbungen konkret in der Wortwahl niederschlagen.

336 Linke/Nussbaumer/Portmann 1996, S. 212 
Die Wortschatzuntersuchung soll mit der nachfolgenden Betrachtung der Organisationsprinzipien (Systemstellen, Wortschatzsektoren und Wortfamilien) beginnen; weiterhin soll anschließend der Versuch unternommen werden, den Wortschatz thematisch und funktional zu untergliedern. Jedoch besteht bei der Untersuchung des politischen (Fach)Wortschatzes "als thematische[m] Wortschatz der betreffenden Fachleute ${ }^{\prime 337}$ grundsätzlich das Problem der Abgrenzung dieses Sektors gegenüber anderen Sektoren des alltäglichen Sprachgebrauchs. Zwar existieren Ausdrücke wie beispielsweise Abgeordneter oder Parlament, die eindeutig dem politischen Bereich zugewiesen werden können, die Politik ist jedoch kein Sachgebiet mit einem klar abgrenzbaren Lexikon, sondern „ein Handeln oder ein Handlungs- und Funktionskomplex. “338 Da grundsätzlich alle Wörter zu politischen Ausdrücken werden können, wenn diese Gegenstand einer politischen Kontroverse oder in eine solche eingebunden sind, ist das politische Lexikon gleichzeitig auch von Fachwörtern anderer Sachgebiete durchdrungen. Diese These kann an der nachfolgend zitierten Textpassage verdeutlicht werden:

(45) (a) Die Präimplantationsdiagnostik (PID) als eine Methode zur Selektion behinder-ten Lebens bei künstlicher Befruchtung lehnen wir $a b$, auch wenn sie für einzelne betroffene Ehepaare eine zusätzliche Entscheidungsoption sein mag. [...] Moderne Reproduktionstechnologie und die Forschung an embryonalen Stammzellen bedrohen zusätzlich das reproduktive Selbstbestimmungsrecht von Frauen; zunehmend laufen Frauen als Eizellenlieferantinnen oder Spenderinnen von Embryonen Gefahr, zum Spielball medizinischer, wissenschaftlicher oder wirtschaftlicher Interessen zu werden. (B 90/GRÜNE 2002, S. 85/138)

Bei den unterstrichenen Ausdrücken Präimplantationsdiagnostik, behindertes Leben, künstliche Befruchtung, Reproduktionstechnologie, Forschung, Embryonen und embryonal, Stamm- und Eizellen, medizinisch sowie wissenschaftlich handelt es sich um medizinische Termini, die in einer öffentlich-politischen Diskussion verwendet werden. Zur Politisierung eines gesellschaftsrelevanten Themas soll eine Äußerung des ehemaligen Bundespräsidenten Johannes Rau (SPD) aus dem Jahre 2001 zitiert werden, die sich auf einen ähnlichen thematischen Sachverhalt bezieht und daher auf die oben zitierte Textpassage der GRÜNEN übertragen werden kann: „Hier wirft die Wissenschaft Fragen auf, die uns alle angehen.

337 Gloning 2003, S. 68

338 Dieckmann, Walther: Sprache in der Politik. Einführung in die Pragmatik und Semantik der politischen Sprache. Heidelberg 1969, S. 47 
Sie müssen in der ganzem Gesellschaft diskutiert und sie müssen dann politisch entschieden werden - im Parlament. “"

Die vorliegende Untersuchung beschränkt sich aufgrund der nahezu unüberschaubaren Masse an Parteiprogrammen (Wahl-, Orientierungsund Grundsatzprogramme in verschiedenen Jahrzehnten von unterschiedlichen Parteien) auf die in der Einleitung genannten Programme, jedoch muss der Untersuchungsgegenstand, insbesondere im Hinblick auf die Wortschatzarbeit, weiter eingegrenzt werden. Daher wird der Schwerpunkt in den nachfolgenden Kapiteln erneut auf dem Godesberger Programm liegen und sich an bestimmten Untersuchungskriterien orientieren; bewährte Ansätze der Wortschatzbetrachtung sind beispielsweise die Untersuchung der Bedeutungszusammenhänge einzelner Elemente, Arten der Wortbildung, Wortfamilien, Fremdwörter oder Lesarten. ${ }^{340}$

\subsubsection{Systemstellen}

Die dieser Untersuchung zugrunde liegenden Parteiprogramme lassen sich nach bestimmten Systemstellen strukturieren, die in den jeweiligen Texten nachgewiesen werden können. Eine solche parteiübergreifende Systemstelle ist unter anderen die, welche Akteure nennt oder beschreibt. Wenn - wie in dieser Analyse bereits ausführlich dargelegt wurde - unter politischer Kommunikation eine spezifische Form sozialen Handelns verstanden wird, dann „,werden die Beteiligten an Kommunikationsprozessen als Akteure gesehen, d.h. als Individuen oder Kollektive, die sym-

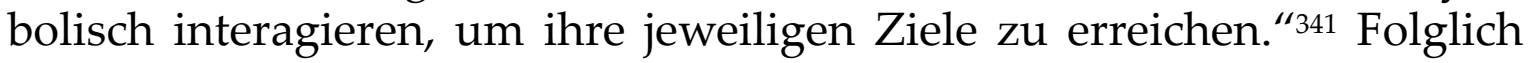
ist der Akteursbegriff dabei nicht auf Individuen beschränkt, sondern umfasst als Handelnde auch Institutionen oder gar Länder und Staaten. Die Systemstelle Akteure kann wiederum untergliedert werden, beispielsweise in Akteure der Politik, des Militärs und der Ökonomie.

Der Unterkategorie Politik lassen sich die folgenden Wörter zuordnen: Partei, Volk, Regierung und Opposition, die Politiker, die Deutschen und die Nicht-Deutschen, wir [sowohl bezogen auf die jeweilige Partei und deren Mitglieder, als auch stellvertretend für alle Menschen bzw. alle Rezipienten (Integrations- und Appellfunktion)], die Kommunisten, die Sozialisten, die Liberalen, die Sozialdemokraten, der Europarat.

Länder und Staaten, die in den Parteiprogrammen genannt werden, bilden eine weitere eigene Unterkategorie. Diese Unterkategorie der

339 Rau, Johannes: „Wird alles gut? Für einen Fortschritt nach menschlichem Maß." Berliner Rede vom 18.05.2001, Kap. I, S. 2

<http://www.bundespraesident.de/top/dokumente/Rede/ix_41073.htm> (14.09.2004)

340 Gloning 2003, S. 58ff.

341 Jarren/Sarcinelli/Saxer 1998, S. 636 
geographischen Orientierungspunkte beinhaltet folgende Wörter und Namen: Deutschland, Polen, Sowjetunion, Tschechoslowakei, Europa, Afrika, Südamerika, Vietnam oder die Bundesrepublik Deutschland. Dennoch sind diese Namen in Zusammenhang mit der Systemstelle Akteure und der Unterkategorie Politik zu nennen, da abhängig vom jeweiligen Kontext einem Land oder einem Staat die Rolle des Agens zugeschrieben wird (stellvertretend für die regierende Partei):

(46) (a) Die Bundesrepublik Deutschland hat Millionen Heimatvertriebene und Flüchtlinge aufgenommen. (CDU 1971, S. 49)

Hinsichtlich der Systemstelle Akteure und der Unterkategorie Politik werden in den Parteiprogrammen auch explizit einzelne Persönlichkeiten des politischen oder gesellschaftlichen Geschehens namentlich erwähnt, wie (Karl) Marx, Scheidemann, Lenin, (Friedrich) Engels, August Bebel, Wilhelm Liebknecht, Ludendorff, Hindenburg, Tirpitz, Karl Liebknecht, Rosa Luxemburg oder Ernst Thälmann. Erwähnt werden muss in diesem Zusammenhang, dass abhängig von der Ideologie der jeweiligen Partei die Namen solcher Persönlichkeiten entweder als Mittel der Distanzierung oder zum Zweck der Identifikation gebraucht werden.

Wie bereits erwähnt kann die Systemstelle Akteure untergliedert werden in den Bereich Militär. Dieser Bereich lässt sich wiederum in die Kategorien Remilitarisierung und ausführende Organe untergliedern. Die Kategorie Remilitarisierung beinhaltet die Wörter Militarisierung und Remilitarisierung, Aufrüstung, Verteidigung und Verteidigungsbeitrag.

Der Kategorie der ausführenden Organe lassen sich die nachfolgend aufgezählten Wörter zuordnen: Polizei und Kriminalpolizei, Armee.

Der Bereich der Ökonomie bildet eine weitere Untergruppe der Systemstelle Akteure. In diesen Bereich fallen die Wörter Industrie, Industriezentren und Industrieländer, Großorganisationen, Großunternehmen, Großwirtschaft.

Die Systemstelle Kommunikation stellt ein weiteres Element zur Strukturierung der Parteiprogramme dar. Die Wörter dieser Systemstelle lassen sich untergliedern in die Bereiche der mündlichen und schriftlichen Kommunikation. Die Ausdrücke Rundfunk und Fernsehen sind dem bereich der mündlichen Kommunikation zuzuordnen, wohingegen Presse und Zeitung dem Bereich der schriftlichen Kommunikation angehören. Andere Wörter können kontextabhängig beiden Bereichen zugeordnet werden, wie beispielsweise Erklärung, Propaganda und Hetze, Debatte, Diskussion und Diskussionsbeitrag, Standpunkt, Beitrag, Vergleich, propagieren, Propagierung und propagandistische Tätigkeit. So kann eine Erklärung oder ein Beitrag sowohl schriftlich als auch mündlich abgegeben werden und eine Diskussion kann ebenfalls schriftlich oder mündlich erfolgen. Aus der Verwendung mancher dieser Ausdrücke in den Parteiprogrammen geht 
nicht hervor, in welcher Form die Diskussion über ein bestimmtes Thema erfolgte. Dies lässt sich an folgender Äußerung verdeutlichen:

(a) Wir sprechen uns konsequent für eine den Einsatz, den Export und die Produk-tion umfassende Ächtung von Landminen aus und treten für eine maximal beschleunigte Beseitigung der weltweit ausgelegten Minen ein. (B 90/GRÜNE 2002, S. 164/165)

(b) Die Verabschiedung des neuen Grundsatzprogramms schloss eine dreijährige Debatte erfolgreich ab. (B 90/GRÜNE 2002, S. 7)

Die GRÜNEN formulieren die oben zitierte Aussage in einem schriftlich fixierten Programmtext und verwenden dennoch das Verb aussprechen. Im eigentlichen Sinn ist jedoch die parteispezifische Ansicht zum Ausdruck gebracht und aussprechen ist nicht ausschließlich dem Bereich der mündlichen Kommunikation zuzuordnen. Es wird hier vielmehr zum Ausdruck gebracht, dass bestimmte Themen innerhalb der Partei „im Gespräch sind“, d.h. konkrete Pläne und Vorhaben realisiert werden sollen. Es wird weiterhin dargelegt welche Haltungen und Ansichten der Rezipient unterstützt, wenn der als Wähler seine Stimme den GRÜNEN gibt. Daher haben die Verben aussprechen und eintreten in Äußerung (a) die gleiche Bedeutung. Der unterstrichene Ausdruck eine dreijährige Debatte erfolgreich abschließen in Äußerung (b) lässt ebenfalls offen, ob in dieser Verwendung die schriftliche oder die mündliche Kommunikation gemeint ist. Da der Verabschiedung eines neuen Grundsatzprogramms jedoch zwangsläufig ein (schriftlicher) Programmentwurf vorausgeht und dieser diskutiert wird, sind in dieser Verwendung wahrscheinlich beide Kommunikationsbereiche angesprochen.

Die Auswahl der genannten Systemstellen erhebt keinen Anspruch auf Vollständigkeit, sondern soll exemplarisch einige signifikante Bereiche der Parteiprogramme aufzeigen. Außerdem finden sich in den Programmtexten - insbesondere in den Bereichen Politik und Militär - große Überschneidungen, die die Zuordnung in eine der jeweiligen Kategorien erschweren. Konkret bedeutet dies, dass sich einzelne Worte mehreren Kategorien zuordnen lassen, beispielsweise als Land, Staat und Agens im militärischen und politischen Sinn. Einige der in den Parteiprogrammen verwendeten Wörter sind aufgrund ihrer Abstraktion nur sehr schwer bestimmten Bereichen oder Systemstellen zuzuordnen, wenn sie isoliert betrachtet werden.

So lassen sich beispielsweise die Untergruppen Ökonomie und Politik schwer unterscheiden. Es geht zwar aus dem Kontext sowie aufgrund der Kapitelüberschrift Agrarwirtschaft die Bedeutung des Ausdrucks sozialdemokratische Wirtschaftspolitik hervor, die Zuordnung zu einer bestimmten Systemstelle gestaltet sich aber schwierig, was in der tatsächlichen Nähe der beiden Bereiche begründet liegen kann. 
Weiterhin lässt sich hinsichtlich der Systemstellen auch keine eindeutige Dominanz erkennen, allerdings scheint den Bereichen Militär und Politik ein hoher Stellenwert von den Parteien eingeräumt zu werden, was im nachfolgenden Kapitel bei der Untersuchung der Wortschatzsektoren zu überprüfen sein wird.

\subsection{Wortschatzsektoren, Wortfamilien und Wortfelder}

In den Parteiprogrammen lassen sich verschiedene Wortschatzsektoren benennen, wie beispielsweise die Sektoren Militär, Wirtschaft, Sicherheit, Politik, Rechtswesen, Öffentlichkeit oder Formen des Zusammenlebens. Diese Sektoren resultieren aus den thematischen Inhalten der Parteiprogramme, da zwangsläufig ein bestimmter Wortschatz benutzt wird, wenn die Parteien in ihren Programmen über verschiedene Themen sprechen. So bilden die Wortschatzsektoren und deren Untergliederungen häufig ein bestimmtes Kapitel bzw. die dazugehörigen Unterkapitel des Parteiprogramms nach. Die Kategorienerstellung ist nicht unproblematisch. Häufig sind Doppelnennungen nötig, da sich insbesondere Abstrakta schwer bestimmten Kategorien eindeutig zuordnen lassen. Es soll nachfolgend dennoch der Versuch unternommen werden, da trotz möglicher Kategorienunschärfe ein Eindruck des Lexikons und seiner Elemente bei der politischen Sprachverwendung in den Parteiprogrammen entsteht.

\section{A Wortschatzsektor Militär}

Ein Sektor, der sich auf den militärischen Bereich bezieht, ist im Text deutlich nachweisbar. Beispiele für die Verwendung von Wörtern des militärischen Bereichs sind Militär, militärisch und nicht-militärisch, Militärgerichtsbarkeit, Luft-, Marine-, Raketen- und Militärstützpunkte, Militärblocks, militärgestützt und militärfixiert, Militärfixiertheit, Militärgewalt, Militärhilfe, Militarismus und Militaristen, Militärapparat und Militärbündnis(se).

Der Bereich Militär kann jedoch in weitere untergeordnete Kategorien untergliedert werden, beispielsweise die Kategorie von Wörtern, die Heeresabteilungen oder die Organisation einer Armee benennen, wie Truppen, Soldat, Reservist, Offiziere und Unteroffiziere, Soldatenräte, Rote Garde, Kriegsdienstverweigerer, Deserteur, Söldnertruppe, Stoßtrupp, Volksheer, Bundeswehr, Armee, Verteidigungstruppe, Miliz und Arbeitermiliz, Militärstrategen, Brigaden, Militärtechniker, Mannschaften, Streitkräfte sowie der Ausdruck Uniform.

Eine weitere Kategorie des militärischen Bereichs ist die der unmittelbaren Kampfhandlungen, wie beispielsweise Krieg, Vernichtungs- und Zerstörungskrieg, Nuklearkrieg, Weltkrieg, Kampf sowie Befreiungskampf und Klassenkampf, Revolution(en) und Gegenrevolution, Aggression, Konfrontati- 
on, Konflikt, Metzeleien, Putsch, Anschläge, Ränke, Zettelungen, Terror, kämpfen und erkämpfen, vernichten, (physische) Vernichtung, zerstören, bedrohen, usurpieren, Hauptkriegsherd, Schlachtfeld, Meuchelmord und politischer Mord, Blutvergießen, Angriff, Marsch und Vormarsch oder Rückschläge.

Die Wörter Militarisierung und Remilitarisierung, Aufrüstung, Atomkriegsvorbereitungen, Wettrüsten, Verteidigung, Zivil- und Landesverteidigung, Verteidigungsfall sowie -gemeinschaft, -bereitschaft, -kraft, -bündnis, -aufgabe und -beitrag, Rüstung sowie der Ausdruck Grenzen wahren lassen sich der Unterkategorie der Wiederbewaffnung oder deren Rechtfertigung zuordnen. Laut WENGELER (1996a) sind Ausdrücke wie Verteidigungsbeitrag oder Remilitarisierung „Bezeichnungen für die Aufstellung einer neuen deutschen Armee. ${ }^{\prime 342}$ Der Debatte um die Wiederbewaffnung der Bundesrepublik sind auch die Ausdrücke Abrüsten, Rüstungskontrolle und Abrüstungsverhandlungen zuzuordnen.

Die Unterkategorie der Mittel zur militärischen Durchführung beinhalten die Wörter Säbel, Waffensystem, Minen und Landminen, Kampfmittel, Raketen, Waffe und Atomwaffen, NATO-Mittelstreckenraketen (sowie als konkrete Bezeichnungen Pershing II und Cruise Missiles), chemische und biologische Waffen sowie Massenvernichtungsmittel.

\section{B Wortschatzsektor Politik}

Der zweite Wortschatzsektor, der sich an den Parteiprogrammen nachweisen lässt, beinhaltet Wörter des politischen Bereichs, wie beispielsweise Partei, Parlament, Bundeskanzler oder Propaganda.

Wie bereits beim militärischen Bereich lassen sich auch hier Unterkategorien zur Gliederung des Sektors benennen. Eine solche Unterkategorie beschreibt Regierungssysteme oder Regierungsformen wie die Ausdrücke Staat, Besatzungsmacht, totalitäres und autoritäres System, Anarchie, Monarchie, Demokratie, Regime, Reich, Diktatur, Herrschaft, Republik, Vorherrschaft und Sozialstaat.

Eine weitere Kategorie umfasst Wörter, die eine Ideologie oder eine politische Richtung beschreiben, wie Sozialismus und sozialistisch, Kapitalismus und kapitalistisch, Weltanschauung, Humanismus, Leninismus, Marxismus und marxistisch, Glaubens- und Denkrichtungen, Faschismus und Hitlerfaschismus, neofaschistisch und antifaschistisch, Nationalsozialismus und nationalsozialistisch, neonazistisch, Demokratie, Kommunismus und Antikommunismus, Antisozialdemokratismus, Imperialismus und Sowjetimperialismus, Revanchismus, Chauvinismus, Liberalismus, Dirigismus, Ideologie, Rechtsextremismus und rechtsextrem, Rechtspopulismus, Rassismus und ras-

342 Wengeler, Martin: „Gleichgewicht im Kalten Krieg. Leitvokabeln der Außenpolitik." In: Politische Leitvokabeln in der Adenauer-Ära. Hg. v. Karin Böke/Frank Liedtke/Martin Wengeler. Berlin/New York 1996a, S. 280 
sistisch, Antisemitismus, Dogmatismus und dogmatisch, Revisionismus und revisionistisch. Die Bezeichnungen von Anhängern dieser Grundüberzeugungen lassen sich ebenfalls nachweisen: Sozialisten, Sozialdemokraten, Kommunisten, Faschisten, Kapitalisten, die Liberalen, Marxisten, Nationalsozialist, Revisionisten.

Wörter zur Bezeichnung der Organisationen in der Politik und der politischen Handlungsträger stellen eine weitere Kategorie dar, wie beispielsweise Partei und Volkspartei, Parlament und parlamentarisch, (politischer) Bundesgeschäftsführer, Grundsatzkongress, Regierung, Opposition, Bundesdelegierte, Bundespräsident, Bundeskanzler, Minister und Ministerin, Ministerpräsidentin, Bundesminister, Landwirtschaftsminister, Ministerräte, Umweltministerium, Bundestag, Bundesrat, Landtag, Vorstand und Bundesvorstand, Fraktionsvorstände, Institution, Verbände, Vorsitzender, Politiker, Ausschuß, Abgeordneter, Bund, Länder [bezogen auf die Bundesländer der BRD], Gemeinden, Einrichtungen, Verwaltung, Selbstverwaltungsorgane, Arbeiterklasse, Bundesinstitut, Bundesanstalt, alliierte Schutzmächte, Zentralrat, Kommission, Fraktion, Delegation und Delegierte.

Ein weiterer Bereich umfasst Ausdrücke zur Benennung von Zusammenschlüssen wie Vereinte Nationen oder Warschauer Pakt, Europäische Gemeinschaft, Europäisches Parlament, Ostblock, Machtblöcke sowie die phonetisch gebundenen Initialwörter (Buchstabenwörter mit silbischer Aussprache) NATO, UNO und das Handels- und Zollabkommen GATT.

\section{Wortschatzsektor Macht}

Der Wortschatzsektor Macht umfasst die Ausdrücke Macht und Übermacht, Kraft und Kräfte, Machtstruktur, Machtmissbrauch und Versklavung, machtvoll und mächtig, Gewalt und Staatsgewalt, Machtverhältnisse, Verfügungsgewalt.

\section{Wortschatzsektor Sicherheit}

Der Wortschatzsektor Sicherheit beinhaltet die Wörter Schutz, Sicherheit, Kontrolle, Einheit, Entspannung, sichern und gesichert, Frieden, Sicherheitssystem, Bewahrung, schutzlos oder Weltfrieden. Als Institution bzw. als Handlungsträger zur Herstellung öffentlicher Sicherheit werden die Ausdrücke Bundesgrenzschutz, Polizei, Schutzpolizei und Kriminalpolizei verwendet.

\section{E Wortschatzsektor Wirtschaft}

Erwartungsgemäß nimmt das Thema Wirtschaft mit allen dazugehörigen Unterkategorien einen hohen Stellenwert in den Parteiprogrammen ein, da mit der wirtschaftlichen Entwicklung eines Landes eine Vielzahl von Folgeentwicklungen einhergehen, die sich wiederum entweder positiv oder negativ auf nahezu alle gesellschaftlichen Bereiche auswirken. 
Dementsprechend lässt sich ein umfangreiches lexikalisches Profil für diesen Bereich erstellen.

In die Kategorie Wirtschaft fallen im Allgemeinen die folgenden Wörter: Wettbewerb, Ertrag, Wirtschaftsentwicklung, Volksbeschäftigung, Wirtschaftspolitik, Volkswirtschaft, Konsumwahl, Zwangswirtschaft, Wirtschaftsgebarung, Markt und Märkte, Groß- und Gesamtwirtschaft, Wirtschaftsgebilde, Wohlfahrtsstaat, Marktbeherrschung und marktbeherrschend, Gemeinwirtschaft, Arbeitsplatzwahl, Arbeitsbedingungen, Erwerbsstreben, Wirtschaftsbürger.

Die erste Unterkategorie bilden daran anknüpfend die an der Wirtschaft Teilnehmenden, die sich wiederum in zwei Gruppen untergliedern lassen, wie (i) diejenigen Wirtschaftsteilnehmer, die Arbeit zur Verfügung stellen sowie die zu dieser Gruppe gehörigen Ausdrücke und (ii) diejenigen, die Arbeit ausüben sowie die zu dieser Gruppe gehörigen Ausdrücke:

(i) Arbeitgeber, Unternehmen, Betrieb und Großbetrieb, Arzneimittelfirmen, Familienbetriebe, Mittelbetriebe, Arbeitgeberverbände, Großunternehmen, Unternehmerinitiative, Kartelle, Betriebsleitung, Betriebsräte;

(ii) Arbeitnehmer (BRD) und Werktätige (DDR), Angestellte, Bauern, Landwirte, Ernährer, Arbeiter, Beamte, Lehrer, Ärzte, Gewerkschaften und Genossenschaften, Genossenschaftswesen, Selbständige, Streikrecht, Arbeitsleben, Arbeiterschaft, Arbeitsbewegung, Richter, Wissenschaftler, Verbraucher, Arbeitnehmerverbände, Prostituierte, Manager, Proletarier, Produktivkräfte, Streikbewegung, Arbeiterräte.

Die zweite Unterkategorie umfasst die verschiedenen Wirtschafts- oder Dienstleistungszweige: Handel und Welthandel, Industrie und industriell, Kohlenbergbau, Eisen- und Stahlindustrie, Pornographie, Arzneimittelindustrie, Gastronomie, Tourismus, Prostitution, Pharmaindustrie und pharmazeutische Industrie sowie Agrarwirtschaft. Insbesondere dem letztgenannten Bereich der Agrarwirtschaft lassen sich die Ausdrücke Bauern, Landwirtschaft, Landwirt, Genossenschaften, Boden- und Pachtrecht, Agrarwirtschaft, Naturfaktoren, Landbevölkerung sowie landwirtschaftlich zuordnen.

Eine dritte Kategorie beinhaltet Ausdrücke, die bestimmte wirtschaftliche Zustände und deren Auswirkungen beschreiben; dies können sowohl positive Zustandsbeschreibungen wie beispielsweise Produktivität, Wohlstand, Reichtum, Gut und Güter, Massenkaufkraft, Wirtschaftsaufschwung, Wirtschaftswachstum, stetiges Wachstum der Wirtschaft, gesamtwirtschaftliche Wachstumsmöglichkeiten, wachstumsfähige Volkswirtschaft, Konjunktur, Konjunkturschwankungen, Konjunkturablauf, Konjunkturentwicklung, Konjunktursteuerung, Hochkonjunktur, konjunkturell, konjunkturgerecht, zyklisch, Produktionskapazitäten, Weltreichtum, Gesamtkonjunktur oder Konjunkturpolitik sein, als auch negative Beschreibungen wie Rezession, Konjunkturschwäche, Konjunktureinbrüche, finanzieller Staatsbankrott, 
stagnierend, Krise oder Konjunkturkrise und damit einhergehend Staatsschulden, Not, Elend und Armut.

Eine weitere Unterkategorie des Wirtschaftsbereichs umfasst die Ausdrücke der Finanzen. Diese Unterkategorie kann zwar auch als eigenständiger Wortschatzsektor betrachtet werden, die Kategorie soll in dieser Untersuchung dennoch als Teilbereich der Wirtschaft verstanden werden. Zu dieser Unterkategorie gehören die Ausdrücke Nationalbudget, Gesamtrechnung, Inflation, Währung, der EURO, Notenbankpolitik, Geldwesen, Hungerlohn, Kreditwesen, Investition, Millionenwerte, Handelsund Preispolitik, Steuern, Sozialprodukt, Finanzen und finanziell, Forschungsetat, Tarifverträge, Arbeitseinkommen, Zoll, Höchstprofit, Vermögensverteilung, Vermögenslose, Vermögenspolitik.

\section{F Wortschatzsektor Rechtswesen}

Dem Bereich des Rechtswesens gehören folgende Ausdrücke an: Gerechtigkeit und Wehrgerechtigkeit, Arbeits- und Sozialgesetzgebung, Arbeitsgesetzbuch, Sozialgesetzbuch, Exekutive, Legislative, Judikative, Rechtsordnung, Recht und Unrecht, Vorrecht, Grundgesetz, Verfassung, Rechtsgedanke, Gesetzgebung, Rechtsprechung, Interessenvertretung, Richter, Rechtspflege, ehrenamtlich, Kriminalstrafen, Strafrecht und strafrechtlich, Straftat und Gewalttat, Gewaltstraftat, Verbrechen und Verbrecher, Mindeststrafe und Höchststrafe, Strafmaß, Gefüngnisstrafe, Straferhöhung, Strafmilderung, Strafvollzug, Strafprozessordnung, straf- und zivilprozessual, Ordnungsrecht, Ordnungswidrigkeitsrecht, kriminell, Rechtsnormen, Entkriminalisierung, Serientäter, Rechtsweg, Gesetze, (Internationaler) Gerichtshof, Rechtsidee, Folter, Todesstrafe, bestrafen, Verhaftung, rechtlos, Rechtsbewußtsein, Menschenrecht, Völkerrecht und völkerrechtlich sowie Volksgruppenrecht.

\section{G Wortschatzsektor Informationszugang und Öffentlichkeit}

In diesen Wortschatzsektor fallen die Ausdrücke Zugang zu Bildungseinrichtungen, Informationen, Publizität, Öffentlichkeit und öffentlich, Computer, Kommunikationssystem, Schriften, Fernsehshows, Trivialliteratur, Presse, Medien, Zeitung, Rundfunk, Fernsehen und Film.

\section{H Wortschatzsektor Formen des Zusammenlebens}

Dieser globale Wortschatzsektor kann untergliedert werden (i) in den Bereich der Gesellschaft und (ii) in den Bereich der Gemeinschaft. Folgende Ausdrücke sind diesen beiden Unterkategorien zuzuordnen:

(i) Gesellschaft und gesellschaftlich, Mensch und Mitmensch, Zivilisation, Generation, Minderheit (explizit: Homosexuelle, Prostituierte, Drogenabhängige, alte und kranke Menschen, Schwule, Lesben, Sozialhilfeempfänger, Zuwanderer, Ausländer, Behinderte, Kranke, ehemalige Strafgefangene, Sinti/Roma, Zigeuner, Jüdinnen und Juden, schwarze Deut- 
sche, ethnische Gruppierungen) und Mehrheit, Familie, Mütter, Gruppen, Schichten, Aktivistinnen und Aktivisten, Klassen, privat, Öffentlichkeit und öffentlich, Frau und Mann, Jugend, Hausfrau, Kinder, Erwachsene, Wohnung, Heimstätte, die Allgemeinheit, das Ganze, Einzelpersönlichkeiten, Individual- und Gesellschaftswesen, Individualität, Individuum, individuell, individualisiert;

(ii) geographisch: Kontinente, Deutschland, Welt, Europa, Zone, Sowjetunion, Tschechoslowakei, USA, Vietnam, Antarktis, Amerika, Asien, Afrika, Lateinamerika, Polen;

national: Nation, Volk, Völker, Gemeinschaft, Bürger, Glied, Gemeinde, bürgerschaftlich, Bürgerinitiativen oder Bevölkerung;

international: Völkerverständigung [bezogen auf gemeinschaftliche Kooperation], Weltorganisation, Vereinte Nationen, Außenwelt, europäisch, international, Zusammenleben der Völker, Weltbevölkerung, Entwicklungsländer, Nationen, Beziehungen, Europa, Verbindungen und Verflechtungen, Völker, Welt, Zusammenarbeit, Staaten, Erde, Menschheit.

\section{Wortschatzsektor Aus- und Weiterbildung}

Der Bereich der Aus- und Weiterbildung umfasst die folgenden Ausdrücke: Bildungseinrichtung, Begabung, Leistung, Aufstieg, Erziehung, leistungsfähig, Ausbildung, pädagogisch, Ausbildungsstätte, Lern- und Lehrmittel, Bildungsweg, Lehrpläne, Schulerziehung, Schulwesen, Lehre, Kurzausbildung, Pflichtschulzeit, Entwicklung, Schulpflicht oder Hochschulreife.

Weiterhin werden in den Parteiprogrammen unterschiedliche Schulformen genannt, wie die Hochschule, die Grundschule, die Berufsschule oder die Oberschule.

Dem Wissenschaftsbereich als Teilbereich der Aus- und Weiterbildung gehören folgende Ausdrücke an: Technik, Gentechnologie, Forschung, Wissenschaft und Naturwissenschaft, Forschungsrat, Forschungsergebnisse und Fortschritt.

\section{Wortschatzsektor Verhalten und Ansichten}

Dem Wortschatzsektor Verhalten werden die folgenden Ausdrücke zugerechnet: Intoleranz, Toleranz und tolerant, Versagen, Barbarei, solidarisch, kriminell, sozial, modern. Die Abgrenzung dieses Sektors zu möglichen Unterkategorien fällt jedoch schwer, denn auch hier tritt erneut eine gewisse Kategorienunschärfe zutage; so beschreibt der Ausdruck Versagen vielmehr einen Mangel an persönlicher Fähigkeit als ein bestimmtes Verhalten.

Die Grundwerte bilden einen Teilbereich des Sektors Verhalten, da sie das Verhalten in der Gesellschaft beeinflussen. Dazu gehören Solidarität, Freiheit, Gerechtigkeit, Chancen, Freiheit, Würde. 
Weiterhin wirken sich auch moralische Grundüberzeugungen bzw. Ansichten sowie deren Mangel auf das Verhalten aus; in diesen Bereich gehören die Ausdrücke Ethik, Gewissen, Ehrgefühl, Gewissensgründe, uneigennützig, großzügig. Zu den individuellen positiven Grundüberzeugungen und Verhaltensweisen zählen beispielsweise Selbstverantwortung, Entwicklung und Entfaltung der Persönlichkeit, Verantwortungsbewusstsein, Menschlichkeit, Fairness, Würde, Achtung, Vernunft, Bürgersinn, Selbstdisziplin zu den negativen zählen dagegen Stumpfsinn, Gleichgültigkeit, Verständnislosigkeit, Verrohung, Egoismus und Korruption.

\section{K Wortschatzsektor Gesundheitswesen}

Folgende Ausdrücke lassen sich dem Wortschatzsektor des Gesundheitswesens zuordnen: Krankenhausversorgung, Gesundheit, gesundheitlich, gesundheitserhaltend, ärztliche Hilfeleistung, Medikamente, Arzneimittel, Krankenversicherung, Heilmethoden, Psychotherapie, Patient und Patientin, medizinisch, Patienteninteressen, Schulmedizin, schulmedizinisch, Gesundheitsfürsorge, Gesundheitssystem, Diagnose, Therapie, therapeutisch, Behandlung, Apparatemedizin, Medikamentenmarkt, Psychosomatik, Gesundheitskosten, Gesundheitswesen, Sterilität, Fruchtbarkeit und Unfruchtbarkeit, psychisch, physisch, Drogenabhängige, Geschlechtskrankheiten, Stress, psychosozial, chronisch, Tod, Leben, gesundheitsförderlich, Rehabilitation, Gesundheitsförderung, Medizin, Gesundheitsdienst, gemeindepsychiatrisch, Psychiatrie-Enquete, Gesundheitserziehung, Volksgesundheit, Embryo und Embryonen, Gentests, embryonal, Gesundheitsberatung, krankheitsverursachend, Gesunderhaltung, Gesundheitsprävention, Gesundheitsinvestitionen, Behandlungsmethoden, Präimplantationsdiagnostik, Pränataldiagnostik, in-vitro-Fertilisation, Keimbahn, Fitness, Gesundheitssicherung, Infektionen, Schwerverletzte, chirurgisch, Eingriff, Gesundheitsvorsorge, Gesundheitsaufklärung, Intensivmedizin, Psychochirurgie, Chemotherapie, ambulant, Symptomtherapie, Arzneimitteleinsatz, fit, Abwehrkräfte, Ganzheitsmedizin, heilen, Arzneimittelverbrauch, Arzt-Patienten-Verhältnis, Patientendaten, Arzneien, Krebskataster, Naturheilkunde, Krankheitsbewältigung, Stammzellen, Heilung und Heilkraft.

Dieser Sektor lässt sich untergliedern nach Berufen oder Akteuren im Gesundheitswesen: Pflegepersonal, Medizinausbildung, Heilberufe, Gesundheitsberufe, Ärzte, Allgemeinärzte, Therapeuten, Zahnärzte, Hebammen und Krankenschwestern.

Es lassen sich auch die Institutionen im Gesundheitswesen benennen, wie Psychiatrie, Irrenanstalten, Ambulatorien, Kliniken, sanitäre Einrichtungen, Rehabilitationseinrichtungen, Tageskliniken, Einzel- und Gruppenpraxen, Großkrankenhäuser, Krankenkassen, Krankenhaus, Krankenhausträger, Gesundheitszentren sowie Gesundheitsinformationsdienste.

Eine weitere Unterkategorie beinhaltet Gesundheitszustände und Krankheitserscheinungen: Alkoholismus, Neurosen, Massenkrankheiten, Psychosen, Gesundheitsprobleme, Massenerkrankungen, Lungenkrankheiten, 
Schwangerschaft, Erkrankungen der Bewegungsorgane, Lärmschwerhörigkeit, Haut-, Magen- und Darmerkrankungen, Infektionskrankheiten, Zivilisationskrankheiten, Herz- und Kreislauferkrankungen, Diabetes, Krankheitssymptome, Geschlechtskrankheiten, Erbkrankheiten, Erbkranke, Übergewicht, Alkoholund Nikotinmissbrauch, Strahlenkranke, Krankheitsursachen, Sucht, Süchte, Suchtformen, Abhängigkeit, Migräne, Angina pectoris, gynäkologische Beschwerden, Siechtum, Krankheit und Behinderung, behindert sowie Krankheitsfall.

Neben der Einteilung der Elemente, die dem politischen Lexikon angehören, kann es neben den Sektoren auch hilfreich sein, Elemente nach Wortfamilien zu sortieren. Die Grundlage für Wortfamilienzusammenhänge bilden dabei „Wortbildungsmuster, mit denen die Sprecher im Lauf der Sprachgeschichte neue Ausdrücke gebildet haben", und die Gesamtheit dieser Ausdrücke als Wortbildungsformen „kann man ansehen als eine sprachliche Ressource, die den Sprechern zur Bewältigung neuartiger kommunikativer Aufgaben zur Verfügung steht. “343

Nachfolgend sollen exemplarisch zwei umfangreiche Wortfamilien dargestellt werden. Unter Berücksichtigung aller Parteiprogramme lassen sich folgende Ausdrücke einer Wortfamilie zuschreiben: Funktion und die mit dieser Komponente zusammengesetzten Komposita Rest-, Autoritäts-, Teil-, Start-, Lebens-, Vorbild-, Austausch- und Behördenfunktion, Funktionsräume und Funktionsfestlegung, Gewerkschafts-, Staats- und Verbandsfunktionäre, Funktionärsschicht, Funktionalität und Monofunktionalität, Funktionsfähigkeit und funktionsfähig, funktionalisieren, fungieren, funktionieren und substantivisch das Funktionieren, funktionierend, multifunktional, funktionstüchtig, funktionsgerecht sowie funktionell und unfunktionell.

Eine weitere Wortfamilie beinhaltet die Wörter Individuum und Individuen, Individualismus, Individualisierung und Individualisierungsprozess, Individualität und Individualitätsprinzip, individual, individuell, individualisiert, individualistisch sowie die Komposita Individualeigentum, -prinzip, -verkehr und -wesen.

Deutlich wird bei der Betrachtung der zitierten Wortfamilien-Komposita, dass einige der Ausdrücke als Resultate von „lexikalischen Bewältigungsstrategien für neuartige kommunikative Aufgaben“344 betrachtet werden können; somit sind auch Wortfamilien dynamisch und bilden aufgrund ihrer zeitlichen Entwicklung bestehende gesellschaftliche Situationen oder Verhältnisse ab.

Weiterhin kann die Untergliederung des politischen Lexikons nach Wortfeldern der lexikalischen Profilierung dienlich sein. KLEIN (1989) versteht unter den Terminus des Wortfeldes „Begriffsnetze, mit denen man die

343 Gloning 2003, S. 66

344 Gloning 2003, S. 39 
politisch-soziale Welt deutend, wertend und programmatisch überzieht. " ${ }^{345}$ So lassen sich in der Geschichte der Bundesrepublik Deutschland bestimmte Phasen benennen, in denen charakteristische Ausdrücke zu dominierenden Wortfeldern zusammengefasst werden können.

Solche Wortfelder resultieren zum einen aus dem thematischen Schwerpunkt (i) des gesamten Parteiprogramms (wie etwa ein umfangreiches Wortfeld Umwelt bei den GRÜNEN 1980 und B 90/GRÜNE 2002 oder Die Frau in der Gesellschaft bei den FRAUEN 2002), sowie (ii) einzelner Themenabschnitte eines Parteiprogramms (dabei kann das Wortfeld die Titel der jeweiligen Kapitel häufig wörtlich oder leicht angepasst übernehmen, wie etwa Grundwerte des Sozialismus, Grundforderungen, Die staatliche Ordnung, Landesverteidigung, Wirtschafts- und Sozialordnung, Eigentum und Macht, Einkommens- und Vermögensverteilung, Agrarwirtschaft, Gewerkschaftswesen, Soziale Verantwortung, Das kulturelle Leben, Die Wissenschaft, Die Kunst oder Internationale Gemeinschaft im Godesberger Programm 1959). KLEIN (1989) formuliert beispielsweise ein Wortfeld der außerparlamentarischen Linken, ein Wortfeld der frühen sozial-liberalen Koalition der Ära Brandt, ein Wortfeld der Ökologie bzw. der ökologischen Bewegung, ein Wortfeld, das Ausdrücke der Protest-Bewegungen der 1980er Jahre umfasst oder ein Wortfeld Sprache der Wende. ${ }^{346}$

Hinzuweisen ist an dieser Stelle auf den Band Politische Leitvokabeln in der Adenauer-Ära von BÖKE/LIEDTKE/WENGELER (vgl. Bibliographie).

Bei der Analyse des politischen Lexikons als Teilausschnitt des Gesamtwortschatzes kann es darüber hinaus dienlich sein, die Elemente des politischen Wortschatzes nach Gruppen zu ordnen. Nachfolgend soll der etablierte Ansatz von DIECKMANN (1969) berücksichtigt und mit Beispielen der untersuchten Parteiprogramme unterfüttert werden. So können die Elemente des politischen Lexikons nach den Bereichen (i) der "Institutionssprache“, (ii) der „Fachsprache des verwalteten Sachgebiets“ sowie (iii) der "Ideologiesprache“ untergliedert werden. ${ }^{347}$

\section{(i) Institutionsprache}

Die Institutionssprache setzt sich nach DIECKMANN (1969) zusammen „aus den Bezeichnungen für die einzelnen Institutionen und Organisationen eines Gemeinwesens, ihre interne Gliederung, die Aufgaben, die sie erfüllen und die Prozesse, in denen sie funktionieren [...]" und regelt "das formale Funktionieren der Institution, Behörde, Organisation zur Erledi-

345 Klein, Josef: „Wortschatz, Wortkampf, Wortfelder in der Politik.“ In: Politische Semantik. Beiträge zur politischen Sprachverwendung. Hg. v. Josef Klein. Opladen 1989, S. 29

346 Klein 1989, S. $29 \mathrm{ff}$.

347 Dieckmann 1969, S. 50 
gung der Aufgaben, die sie von der Verfassung oder der übergeordneten Autorität erhalten haben. “348

Das „Institutionsvokabular“349 dieser Kategorie umfasst im Wesentlichen die bereits unter $B$ Wortschatzsektor Politik aufgelisteten Ausdrücke; der Bereich der Institutionssprache lässt sich wiederum untergliedern in die "Organisationssprache" einerseits und die "Verfahrenssprache" andererseits. Zur ersteren gehören die Bezeichnungen für die Organisationen und Institutionen selbst (Formula als Fachvokabular), wie Bundestag, Bundesrat, Landtag, Bundesrepublik (Deutschland), Parlament, parlamentarische Demokratie, Bundesinstitut, Ministerien, Bundesanstalt oder Partei; deren Gliederungen wie Kommission, Delegation, Vorstand und Bundesvorstand, Fraktion und Fraktionsvorstände, Ausschuß; deren Aufgaben wie Verfassungsschutz, Finanzpolitik oder Entwicklungshilfe sowie deren staatliche oder politische Rollen und Amtsbezeichnungen wie Bundespräsident, Bundeskanzler, Minister und Ministerin, Ministerrat, Ministerpräsidentin, Bundesminister oder Bundesgeschäftsführer.

Der verfahrenssprachliche Wortschatz beinhaltet die Bezeichnungen für die formalen Praktiken, mit denen die Institutionen arbeiten (politische Handlungen, Prozesse und Zustände); dazu gehören die Ausdrücke Tagesordnung, Parteitag, Wahl, Tagung, Volksbegehren, Volksentscheid, Legislaturperiode, Debatte, Diskussion, Quotierung, Wahl oder Abstimmung. KLEIN (1989) formuliert eine weitere Unterkategorie, die „Ausdrücke für kodifizierte Normierungen politisch institutionellen Handelns “350 umfasst; dazu gehören Ausdrücke wie Grundgesetz, Programm, Statut, Pakt, Staatsvertrag oder Verfassung.

Auffällig ist in den untersuchten Parteiprogrammen, dass die Bezeichnung für die Bundesrepublik Deutschland als Kurzform (Akronym) BRD lediglich in den Programmen der GRÜNEN 1980 und der FRAUEN 2002 verwendet wird; die übrigen Parteiprogramme verwenden die Bezeichnung in der Form der Wörter Bundesrepublik oder Deutschland oder beiden zusammen, während die Abkürzung DDR häufig verwendet wird. Dieser Sachverhalt lässt sich folgendermaßen erklären: „Politische Sprachkontrolle (z.B. die Verpöntheit des Ausdrucks BRD für unser Gemeinwesen) dient der sozialen und ideologischen Kontrolle von Großgruppen. “351

Die Abkürzung BRD, die in der DDR erst seit 1969 im öffentlichen Sprachgebrauch verwendet wird und in den 70er Jahren in der Bundesrepublik als ,kommunistische Erfindung' und ,Agitationsformel'

\footnotetext{
348 Dieckmann 1969, S. 50/51

349 Klein 1989, S. 5

350 Klein 1989, S. 5

351 Greiffenhagen 1980, S. 12
} 
verfemt ist, wird in den 50er Jahren vor allem in Lexika und amtlichen Schriften verwendet und war noch ,in keiner Weise anstößig'. Im öffentlich-politischen Sprachgebrauch spielte die Abkürzung in den 50er Jahren noch keine Rolle. ${ }^{352}$

\section{(ii) Fachsprache des verwalteten Sachgebiets}

Die Fachsprache des verwalteten Sachgebiets gliedert sich nach den jeweils behandelten Themengebieten. So können bestimmte Wortschatzeinheiten (Wortschatzsektoren) benannt werden, wie beispielsweise die Wirtschaftspolitik, das Militärwesen, die Kulturpolitik oder das Rechtswesen. Nach DIECKMANN (1969) entsteht eine politikeigene Sondersprache der politischen Experten, wenn fachsprachliche Elemente eines bestimmten Lexikons in einen politischen Diskurs eingelagert werden, d.h. wenn die verschiedenen Sachgebiete staatlich verwaltet werden:

Dort vor allem verbinden sich die Fachsprache des Sachgebietes, in diesem Fall die Fachsprache der Wirtschaft, die als solche außerhalb des politischen Bereichs steht, mit der Institutionssprache zu politikeigenen Neubildungen und Zusammensetzungen, die wir mit dem Begriff der Fachsprache des verwalteten Sachgebietes bezeichnen, um sie von der Fachsprache der Wirtschaft abzugrenzen. ${ }^{353}$

Ausdrücke, die der Fachsprache des verwalteten Sachgebiets zugeordnet werden können, sind im wirtschaftlichen Bereich beispielsweise Konjunktur, Mineralölsteuer, Bruttosozialprodukt, Zahlungsbilanzen oder soziale Marktwirtschaft. Die Merkmale dieses „Ressortvokabulars“354 stimmen mit denen einer echten Fachsprache nahezu überein: „Sachlichkeit, Rationalität, Eindeutigkeit, Ökonomie und Variationsarmut. “355 Jedoch veranschaulicht insbesondere der letztgenannte Ausdruck soziale Marktwirtschaft, dass ein Ausdruck bei Eingang in ein politisches Lexikon das Merkmal der Ideologiegebundenheit erhalten kann.

\section{(iii) Ideologiesprache}

Hinsichtlich der Ideologiesprache ist grundsätzlich zu erwähnen, dass jeder Wortschatz, d.h. jedes "Ideologievokabular" ${ }^{\text {“36 }}$ eines bestimmten Herrschaftssystems eine getrennte Behandlung erfordert. So lassen sich beispielsweise im Parteiprogramm der NSDAP als Partei mit nationalsozialistischer Prägung andere ideologiegebundene Ausdrücke nachweisen als im Parteiprogramm der SED, der DKP oder der KPD mit marxis-

\footnotetext{
352 Wengeler 1996b, S. 368

353 Dieckmann 1969, S. 51

354 Klein 1989, S.6

355 Dieckmann 1969, S. 52

356 Klein 1989, S. 7
} 
tischem Entstehungshintergrund (so finden sich in deren Parteiprogrammen Ausdrücke (Credenda) wie beispielsweise Ausbeutung, Kapitalist, Imperialist, Klassenkampf, Volkseigentum, volkseigene Betriebe oder Planwirtschaft). Diese Voraussetzung wird insbesondere bei der späteren Untersuchung der Schlagworte zu berücksichtigen sein. Der lexikalische Kernbestand der Ideologiesprache ist zumeist philosophischen oder gesellschaftstheoretischen Ursprungs und hat insofern oftmals fachsprachlichen (terminologischen) Charakter.

Nachfolgend soll das Parteiprogramm der NSDAP von 1920 auf ideologiegebundene Ausdrücke untersucht werden. Folgende Ausdrücke sind darin nachweisbar: Führer, Groß-Deutschland (Bezeichnung der Nationalsozialisten für „ein Deutsches Reich, dessen Gebiet nach ihrer Forderung größer sein sollte, als es nach dem Ende des von Deutschland verlorenen Ersten Weltkrieges, 1914-1918, im Versailler Vertrag von 1919 festgelegt worden war" ${ }^{\prime 357}$ ), deutsches Blut, Nicht-Deutscher und nichtdeutsch, Gut und Blut, Rasse, deutsches Gemeinrecht, Fremdengesetzgebung, Volksgesundheit, Volksgenosse („Anrede, die Adolf Hitler in vielen seiner Reden benutzte und die während der Zeit der nationalsozialistischen Herrschaft in Deutschland, 1933-1945, mit gleichbleibender Häufigkeit in Reden und Bekanntmachungen angewendet wurde ${ }^{\prime 358}$ ), Reich, germanische Rasse sowie Bekämpfung des jüdisch-materialistischen Geistes.

Den Kernbestand des Ideologievokabulars bilden die Symbolwörter, die sich wiederum in (i) Leitwörter oder Miranda und (ii) Anti-Miranda als Typen der ideologiegebundenen Lexik untergliedern lassen.

Bei einem Mirandum handelt es sich um einen „ideologiegebundenen sprachlichen Ausdruck, der für die Mitglieder einer Sprachgemeinschaft positive Evaluation besitzt. “" ${ }^{359}$ Miranda können auch parteiübergreifend als positiv bewertet werden; als politische Leitwörter sind sie „Objekte (groß)gruppen- und parteibezogener sprachlicher Usurpationsversuche $^{\prime \prime 360}$ und werden nie als Stigmawörter verwendet (diese werden im nachfolgenden Kapitel Schlagwörter genauer betrachtet). Die Wörter Demokratie, Freiheit, sozial oder Fortschritt gehören der Kategorie der Miranda an; insbesondere das Wort Demokratie kann als reiner Fachbegriff gebraucht werden (in einer politologischen Abhandlung), in der politi-

357 Kammer, Hilde/Bartsch, Elisabet: Nationalsozialismus. Begriffe aus der Zeit der Gewaltherrschaft 1933-1945. Reinbek 1992, S. 84

358 Kammer/Bartsch 1992, S. 223

359 Girnth 2002, S. 53

360 Strauß, Gerhard/Zifonun, Gisela: „Formen der Ideologiegebundenheit: Versuch einer Typologie der gesellschaftspolitischen Lexik." In: Der politische Wortschatz. Zur Kommunikations- und Textsortenspezifik. Hg. v. Gerhard Strauß. Tübingen 1986b, S. 104 
schen Kommunikation kann es aber auch ein Mirandum sein ebenso wie die parteiunabhängigen Ausdrücke freiheitliche Demokratie oder soziale Demokratie.

Als Anti-Miranda werden Ausdrücke bezeichnet, die „in beiden Kommunikationsgemeinschaften/ideologischen Systemen A und B gebraucht und von der Ideologie beider Gesellschaftssysteme gleichermaßen abgewiesen werden, [...]." ${ }^{\prime 361}$ Anti-Miranda stellen die Negation der Leitwörter (Miranda) dar; Ausdrücke wie Faschismus oder Aggression werden als Anti-Miranda parteiübergreifend negativ bewertet und verhalten sich somit spiegelbildlich zu einem Mirandum wie Demokratie.

\section{(iv) Allgemeines Interaktionsvokabular}

KLEIN (1989) greift die oben dargestellte Gliederung auf und erweitert die Kategorien um den Bereich des „allgemeinen Interaktionsvokabulars" als "allgemeinsprachliche[] Bezeichnungen für menschliche Interaktion und ihre verschiedenen Aspekte ${ }^{\prime 362}$. Diese Kategorie umfasst Ausdrücke, die weder fachsprachlich noch ideologiesprachlich sind, wie beispielsweise Auseinandersetzung, gefährden und Gefährdung, beteiligen und Beteiligung, planen, Plan und Planung, (Gesamt-)Konzept, Konzeption, Kompromiss, Kraftanstrengung, Beschluss, verhindern, Änderung, Schaden, Opfer, Vergeltung, Mobilisierung, Krise, verschärfen, Angriff, Sorge sowie Krisenprävention.

In diesen Bereich gehört auch ein umfangreiches Vokabular zur Bezeichnung sprachlicher Handlungen: fordern und Forderung (stellen), begründen und Begründung, kritisieren und Kritik, begrüßen, anregen und Anregung, beseitigen und Beseitigung, warnen (auch in substantivierter Form das Warnen), erklären und Erklärung, appellieren und Appell, fragen und Fragen, drohen und Drohung, bedrohen und Bedrohung, versprechen und Versprechen, bilanzieren, Bilanz und Bilanzierung, ankündigen und Ankündigung(en), verkündigen und Verkündigung(en), informieren und Information, widerstehen und Widerstand, unterstützen und Unterstützung sowie vorbehalten und Vorbehalt.

In den Parteiprogrammen lassen sich weiterhin Ausdrücke zur Bezeichnung sprachlicher Interaktionen nachweisen: Streit, streiten und erstreiten, Erörterung und erörtern, Debatte und debattieren, Diskussion und diskutieren, Verhandlung(en) und verhandeln, Einigung und (sich) einigen sowie die Ausdrücke Fragen aufwerfen und Vorschlag machen/unterbreiten.

\footnotetext{
361 Strauß 1986b, S. 104
}

362 Klein 1989, S. 7 


\subsubsection{Erscheinungsformen von Wortbildungsprozessen}

\subsection{Komposita}

Hervorragendes Prinzip der Konzeption der untersuchten Parteiprogramme ist die Verwendung von Komposita, was sich an den Beispielen Strukturanpassungsprogramm, Prostituiertenselbsthilfeprojekte, Zerstörungskriege, Massenvernichtungsmittel, Beschäftigungs- und Lebensrealität, Weltanschauungsgemeinschaften, Bundesumweltministerium, Landschaftspfleger, Länder- und Bundesebene, Natur- und Umweltschutzverbände, Raumordnungsverfahren, Verbandsklagerecht, Sondergenehmigungen, Entscheidungsprozesse, Rüstungskontrollgremien, Planungsverfahren, Großmülldeponien, Landschaftsschutzgebieten, Flurbereinigungen, Erholungsflächen, Bebauungsplänen, Schutzzonen, Grünanlagen, Quellenschutzgebiete, Verkehrsgroßprojekte, Großflughäfen, Großkanäle, Autobahnstrecken, Kostenerstattung oder Reallöhne und Realpolitik belegen lässt.

Aufgrund der Häufigkeit in den Parteiprogrammen ist die Komponente Welt- bei der Bildung von Komposita auffällig. Folgende Wortverbindungen lassen sich in den Parteiprogrammen nachweisen: Weltinnenpolitik, Weltgesellschaft, Weltorganisation, Weltgeltung, Weltmarkt, Welthandel, Weltniveau, Weltsystem, Weltfrieden, Weltbank, Welterfahrung, Weltmaßstab, Weltimperialismus, Welthandelsorganisation, Weltwirtschaftsordnung, Weltgesellschaft, Weltreichtum, Weltbevölkerung, Weltkonzern, Weltherrschaft und Weltherrschaftsanspruch, Weltstruktur, Weltsicherheitsrat, Weltsozialpolitik, Weltmacht, Umweltaußenpolitik, Weltproletariat, Weltgenossenschaft, Weltwirtschaft und Weltwirtschaftssystem.

Wie in den vorangegangenen Untersuchungspunkten zeigt sich auch hier erneut die Schwierigkeit, komplexe Wörter mit einem hohen Grad an Abstraktion isoliert zu betrachten und deren Sinn nachzuvollziehen, wie bei den Ausdrücken Weltstruktur, (sozialistisches) Weltsystem oder Weltimperialismus, die im Parteiprogramm der SED antagonistisch gebraucht werden. So lässt sich der Ausdruck Weltimperialismus auf zweifache Weise interpretieren: (i) als eine imperialistische Weltherrschaft oder Regierungsform, die sich über die gesamte Welt erstreckt und von einem Land oder einem Zusammenschluss von Ländern ausgeht und sich auf andere Länder als Zeichen des Machtstrebens ausdehnt, oder (ii) als die Gesamtheit aller Länder oder Staaten der Welt mit imperialistischem System. Ähnlich unscharf ist auch die Bedeutung des Ausdrucks der (kommunistischen) Weltbewegung.

Hinsichtlich des Wortbildungsprinzips ist generell festzuhalten, dass das Grundwort (Determinatum) durch das Hinzufügen eines Bestimmungswortes (Determinans) näher spezifiziert wird. Die durch diesen Prozess entstandenen so genannten Determinativ-Komposita sind daher 
notwendig zweigliedrig: „Das Erstglied (,Bestimmungsglied') determiniert das Zweitglied, das auch ,Grundglied' genannt wird." ${ }^{363}$ Eine weitere Kategorie bilden die Kopulativ-Komposita, die nicht notwendig zweigliedrig sind und bei denen die einzelnen Glieder einander verbindend (kopulativ) zugeordnet werden, allerdings mit der Einschränkung, dass sich nur Vertreter derselben Wortart additiv verbinden lassen; Ausdrücke wie Freund-Feind-Denken, Nord-Süd-Aktion, Nord-Süd-Politik, NordSüd-Gegensatz, Ost-West-Gegensatz oder Ost-West-Konflikt sind in den untersuchten Parteiprogrammen jedoch marginal.

Die Kategorie der Determinativ-Komposita überwiegt deutlich in den Parteiprogrammen. Es können an dieser Stelle jedoch lediglich einige exemplarische Beispiele angeführt werden, wie die Ausdrücke Eheprozeßrecht, Gerichtsverfassungsgesetz, Raumordnungsregeln, Ostpolitik, Schädlings- oder Unkrautbekämpfungsmittel, Boden-, Steuer-, Gesellschafts-, Bildungs-, Verwaltungs-, Finanz-, Schul- oder Hochschulreform; folglich handelt es sich jeweils um ein bestimmtes Recht, ein Gesetz, eine Regel, eine Politik oder ein Bekämpfungsmittel. Auch geographische Gebiete werden durch Hinzufügen eines Bestimmungswortes voneinander abgegrenzt, wie beispielsweise Mittel-, Ost-, West- und Südosteuropa, Westberlin, Westdeutschland, Ostdeutschland oder Westzonen.

Aus den oben angeführten Beispielen geht hervor, dass innerhalb der Kategorie der Komposita Nominalkomposita vorherrschend sind und unter diesen wiederum solche, die aus zwei Nomen zusammengesetzt sind, wie beispielsweise Machtblöcke, Landesverteidigung und Rechtsordnung. Der überwiegende Teil der in den Parteiprogrammen verwendeten Komposita besteht aus Wörtern, die aus dem Prozess einer Transformation von syntaktischen Wortverbindungen resultieren (Prozess der Konzentration $\rightarrow$ Konzentrationsprozess; Faktor, der etwas über den/einen Standort aussagt $\rightarrow$ Standortfaktor; Verhältnis der Kräfte $\rightarrow$ Kräfteverhältnis; Merkmale des Wissens und der Bildung $\rightarrow$ Wissens- und Bildungsmerkmale; Kreisläufe der Wirtschaft $\rightarrow$ Wirtschaftskreisläufe; Bereitschaft zum Risiko $\rightarrow$ Risikobereitschaft; Fähigkeit zur Veränderung $\rightarrow$ Veränderungsfähigkeit; Gewalt, die vom Staat ausgeht oder die beim Staat liegt $\rightarrow$ Staatsgewalt).

Insbesondere die letztgenannten Beispiele veranschaulichen den Prozess der Wortbildung. Die Bestimmungswörter (Determinans) Konzentration, Standort, Kräft(e), Wissen und Bildung, Wirtschaft, Risiko, Veränderung sowie Staat(s) spezifizieren die Grundwörter Prozess, Faktor, Verhältnis, Merkmale, Kreisläufe, Bereitschaft, Fähigkeit und Gewalt. Dabei werden die Grenzen der zusammengefügten Glieder häufig durch lautliche Fugenelemente (Interfix) markiert. Durch das Hinzufügen von -e- oder -s- wird

363 Vater, Heinz: Einführung in die Sprachwissenschaft. München ${ }^{4} 2002$, S. 77 
die Artikulation des jeweiligen Kompositums erleichtert; Beispiele hierfür liefern die Ausdrücke Aktionsmöglichkeiten, Parlamentsreform, Gewissensgründe, Wirtschaftswachstum, Bewußtseinsveränderung, Richtungsänderung, Willensbildung und Bildungswillen, Gründungsparteitag, Leistungswillen, Volkswillen, Sozialversicherungsrecht, Sozialstaatsgebot, Staatsbürger, Lebensbereich, Bodenrechtsordnung, Eigentumsform, Versorgungsberechtigte.

In diesem Zusammenhang ist allerdings zu erwähnen, dass in den untersuchten Parteiprogrammen einige Ausdrücke als syntaktische Wortverbindungen bestehen bleiben und nicht in Komposita transformiert werden, obwohl diese etabliert und durchaus geläufig sind, wie beispielsweise Verhinderung von Konflikten (nicht Konfliktverhinderung), Entfaltung der Persönlichkeit (nicht Persönlichkeitsentfaltung), aus Gründen der Gewöhnung (nicht aus Gewöhnungsgründen), Erhaltung und Pflege der Natur (nicht Naturerhaltung oder Naturpflege), Sicherung des Friedens (nicht Friedenssicherung), Kontrolle der Regierung (nicht Regierungskontrolle) oder Kampf der Arbeiterklasse (nicht Klassenkampf, obwohl der Ausdruck Klassenkampf im Parteiprogramm der SED 8-mal gebraucht wird).

Neben den Nomen können auch Wörter anderer Wortarten am Prozess der Wortbildung beteiligt sein, wie Verb und Adjektiv. So finden sich in den Parteiprogrammen Nominalkomposita, die aus einem Adjektiv und einem Nomen zusammengesetzt sind, wie Sozialdemokratie, Sozialplanung, Stumpfsinn, Realeinkommen, Reallöhne, Sozialstaat, Sozialabgaben, Sozialleistungen, Sozialstationen, Sozialversicherungen, Produktivkräfte und Produktivkapital, Altlasten, Privateigentum, Rot- und Schwarzwild, Grünflächen, Spezialorganisationen, Intensivmedizin, Zivildienst, Schwarzarbeit oder Schwarzmarkt. Insbesondere die beiden letztgenannten Ausdrücke Schwarzarbeit, Schwarzmarkt oder Schwarzgeld verdeutlichen, dass die Komponenten eines Kompositums die eigenen Bedeutungen verlieren können und das Kompositum eine konventionell festgelegte Gesamtbedeutung erhalten kann.

Dagegen sind die Wörter Daseinsvorsorge, Wohnform, Leiharbeit, Hörfunk, Denkrichtung, Denkgewohnheiten, Denkweisen, Wehrdienst, Denkanstösse oder Denkmuster Nominalkomposita, die aus einem Verb und einem Nomen bestehen.

In den Parteiprogrammen finden sich weiterhin die aus Nomen und Adjektiv zusammengesetzten Adjektivkomposita bündnisgrün (auch in substantivischer Verwendung Bündnisgrüne), zielklar oder Bekanntgabe sowie Verbalkomposita, die sowohl aus Nomen und Verb wie Wertedenken, Obrigkeitsdenken oder Fortschrittsdenken, als auch aus Adjektiv und Verb bestehen, wie klarmachen oder bekanntgeben. 
Als Resultat eines Wortbildungsprozesses sind auch die in den Parteiprogrammen verwendeten Adjektive völkerrechtlich, gesamtwirtschaftlich oder staatsbürgerlich zu betrachten, jedoch greift in diesem Fall nicht das Prinzip der Zusammensetzung (Komposition), sondern das Prinzip der Ableitung (Derivation). Durch die Affigierung bestimmter Derivationsaffixe wie un-, -bar, -keit, -heit, -ung oder -lich an den Stamm des Wortes werden neue Wörter gebildet; dabei bestimmt das jeweilige Affix die Wortart des Lexems, auf das es hinführt.

\subsection{Substantivierungen}

(48) (a) Er verlangt Umdenken, Umsteuern, Auswählen und Gestalten, vor allem in Technik und Wirtschaft. (SPD 1989, S. 7)

Die Substantivierung kann als Wortbildungsprozess verstanden werden. Dabei wird ein Wort oder eine Wortgruppe ohne Verwendung von Affixen in eine andere Wortart überführt. Grundsätzlich ist im Deutschen die Substantivierung von Vertretern aller Wortarten möglich. Bei der intensiven Bearbeitung der Parteiprogramme fallen neben möglichen Wortschöpfungen auch einige ungewöhnliche Substantivierungen von Verben auf. Als Beispiele hierfür können neben Umdenken, Umsteuern, Auswählen und Gestalten in Äußerung (a) die Ausdrücke das Wettrüsten, das [...] Schaffen, das/dieses Denken, das [...] Fortschreiben, das Zusammenleben, das Schweigen, ein [...] Herangehen, das Regierungshandeln, das Verwalten, das Versagen, das Scheitern und das [...] Handeln gelten. Die Ausdrücke neue Form des Wirtschaftens oder zum Verlassen gezwungen sein können ebenfalls als Substantivierungen betrachtet werden.

Weitere gebräuchlichere Substantivierungen finden sich ebenfalls in den Texten, wie beispielsweise das Ökonomisch-Materielle, ins Private oder Bewahrenswertes.

Weiterhin lassen sich substantivierte Partizipien nachweisen, wie der Andersdenkende, der Andersglaubende oder jeder Befähigte.

\subsubsection{Steigerungen}

Einige der unter dem nachfolgenden Kapitel Attributiver Stil angeführten Beispiele wie das nicht steigerungsfähige einzig in die einzige Voraussetzung oder das einzige Mittel eignen sich auch, um Steigerungsformen im Text nachzuweisen. Die Parteiprogramme enthalten viele Komparative wie mit einem größeren Nutzen, in immer weniger Händen, bessere Ordnung, stärker als bisher, freier, gerechter, solidarischer, eine höhere Qualität sowie korrespondierende Formen wie je machtvoller ... desto größer, je bedrückender ... desto stürmischer/rascher/geringer/demütigender und je gefährdeter ... desto nötiger. 
Weiterhin finden sich in den Parteiprogrammen viele Superlative, wie beispielsweise die letzten Offenbarungen, die stärksten propagandistischen Übertreibungen, mit den neuesten Waffen, größter Leistungen, der größte Idealismus, aufs höchste, jüngste Vergangenheit, im weitesten Sinne, schwächste Stellung, die größte und dringendste Aufgabe, am besten, der beste Weg, in tiefster Armut, entschiedenste Zeichen, zuverlässigste Stütze, wichtigste Mittel, baldigster Untergang, schwerste Zerstörung oder das beste Fundament.

Die Verwendung des Indefinitpronomens jedes in den Äußerungen jedes politische Denken und jedes völkerrechtliche Entgegenkommen kann ebenso als Steigerungsform betrachtet werden, da bei Gebrauch der Wendungen ein Vollständigkeitsanspruch oder Absolutheitsanspruch erhoben wird.

Am Beispiel der Ausdrücke Megaprofite (DIE FRAUEN 2002, S. 7), Superschnellbahn (DIE FRAUEN 2002, S. 18), Superstaat (B 90/GRÜNE S. 155) oder Supergau (B 90/GRÜNE S. 30) kann gezeigt werden, dass Steigerungen auch durch das Hinzufügen bestimmter Affixoide wie mega- oder super- ermöglicht werden.

Auch die Komposita Großproduktion, Großkonzerne, Großbesitz, Großbanken, Großindustrie und großindustriell, Großmacht, Großmachtstreben, Großmachtinteressen, Großmachtpolitik, Großkapital, Großwarenhäuser, Großdeutschland, Großforschungsvorhaben, Großforschung, Großprojekte, Großvermögen, Großwirtschaft, Großunternehmungen und Großunternehmen, Großchemie, Großkanäle, Großflughäfen, Verkehrsgroßprojekte, Großmülldeponien, Großtechnologie, Großlandwirtschaft, Großsiedlungen, Großgebilde, Großmaschinen, Großeinrichtungen, Großakteure, Großfamilie, Großverbraucher und Energiegroßverbraucher, Großorganisationen, Großkraftwerke sowie Großbetriebe können als gesteigerte Wortformen betrachtet werden, da sie mit dem Präfix groß- ein intensivierendes Bestimmungswort beinhalten. Ähnlich sind auch Ausdrücke wie hocheffizient oder Überkapazität zu bewerten.

Die Verwendung der genannten Komparative und Superlative ist für die Verfasser der Parteiprogramme ein Mittel, bestimmte Sachverhalte zu betonen und zu akzentuieren. Der Gebrauch von Steigerungsformen dient somit der Eigenwerbung oder der Diffamierung des politischen Gegners und spielt somit eine wichtige Rolle bei der Erzeugung von Sichtweisen.

\subsubsection{Kandidaten für Archaismen/Neologismen}

Die nachfolgend aufgezählten Wörter sind solche, die nach heutiger Betrachtung altertümlich wirken oder im heutigen Sprachgebrauch unüblich sind. In den Parteiprogrammen sind viele solcher Wörter auszumachen. Ob es sich allerdings tatsächlich um "echte“ Archaismen handelt, ist an mancher Stelle lediglich zu vermuten. Aufschluss über diese 
Unklarheit mag die vergleichende Betrachtung der Parteiprogramme im geschichtlichen Verlauf geben.

Bei näherer Betrachtung der Beispiele fällt auf, dass nahezu alle aus heutiger Sicht altertümlich anmutenden Wörter durch die damalige politische Situation geprägt und typisch für den dargestellten Zeitraum sind. Nach dem Zusammenbruch des Warschauer Paktes und der Auflösung der "Zweiten Welt" mit der Sowjetunion als Hegemonialmacht in unmittelbarer Nachbarschaft zur Bundesrepublik und dem damit verbundenen Untergang des Realsozialismus sind diese Wörter nur noch sporadisch im aktuellen Sprachwortschatz aufzufinden. Es ist aber anzunehmen, dass einige der angeführten Beispiele im jeweiligen Sprachgebrauch, beispielsweise um das Jahr 1950, durchaus geläufig waren. Aufgrund der wirtschaftlichen Entwicklungen durch Reformen oder des Wegfallens und der Erneuerung politischer Systeme sind Ausdrücke wie Polizeikorps, Volkspolizei, Sowjetunion oder Rote Armee im heutigen Sprachgebrauch nicht mehr geläufig. Die Übergänge zwischen altertümlich anmutenden Wörtern und Wörtern des Ideologievokabulars sind oftmals fließend, wie die Beispiele Produktivgenossenschaften oder Produktivkräfte zeigen. Der Ausdruck Produktivgenossenschaften wird bereits im Eisenacher Programm und im Gothaer Programm verwendet und als Produktionsgenossenschaften von der SED aufgegriffen, während bereits im Godesberger Programm und in den heutigen Parteiprogrammen lediglich von Genossenschaften oder von einem Genossenschaftswesen die Rede ist. Der Ausdruck Produktivkräfte des Godesberger Programms wird im Jahre 1989 von der SPD ebenfalls verwendet wie von der PDS in 2003 und den GRÜNEN in 2002, wobei in diesem Zusammenhang das Wort Humanressource zu erwähnen ist, das gleichbedeutend verwendet wird.

Bei einer öffentlichen Bezugnahme auf einen der zuvor erwähnten Ausdrücke werden heutzutage Wörter der Distanzierung wie damalig, ehemals, ehemalig sowie das Präfix Ex- hinzugenommen; so spricht man von der damaligen Führung der Sowjetunion oder über die damalige DDR (B 90/GRÜNE 2002, S. 151/9).

Die Problematik dieses Kapitels soll am Beispiel des Ausdrucks Ostzone verdeutlicht werden. Der Ausdruck ist in den Parteiprogrammen nicht nachweisbar, als Bezeichnung lange Zeit im Fokus der öffentlichen Diskussion. Der Ausdruck Ostzone ist laut WENGELER (1996b) bereits seit Mitte 1947 etabliert und bezeichnet entsprechend der Einteilung in vier Besatzungszonen (Bizone, Trizone, Westzonenstaat, Westzonen (SED 1963, S. 230)) die spätere DDR.

In Presseberichterstattungen, etwa über die gescheiterte Münchener Ministerpräsidentenkonferenz von 1947, ist fast ausschließlich von der Ostzone die Rede (vgl. Presseüberblick in DIE WELT 7.6.1947). Schon im April 1948 gibt es aber einen ersten Sprachnormierungsver- 
such, mit dem darauf hingewirkt werden soll, diese Bezeichnung zu vermeiden. [...] Dennoch ist die Ostzone Ende der 40er/Anfang der 50er Jahre eine der häufigsten Bezeichnungen für die DDR. Ebenso wie Sowjetzone und SBZ erscheint es in dieser Frühphase in der Öffentlichkeit noch als wenig politisch. [...] Dies ändert sich aber um so mehr Zeit vergeht und um so mehr die DDR auf ihre Existenz als Staat pocht. Das heißt, die Ausdrücke enthalten zunehmend die Implikation der Nichtanerkennung der DDR als Staat, während sie anfangs noch als eher unpolitische geographische Bezeichnung benutzt werden konnten. ${ }^{364}$

Der Bezeichnung Ostzone wird im Jahre 1950 vom damaligen Oppositionsführer Kurt Schumacher (SPD) in einer Rede ${ }^{365}$ verwendet, in der er die Position seiner Partei in der Remilitarisierungsdebatte der Bundesrepublik Deutschland nach Ende des Zweiten Weltkriegs deutlich macht. Es ist folglich davon auszugehen, dass der Ausdruck Ostzone in Schumachers Rede zwar eine umstrittene, aber dennoch geläufige und keine altertümliche Form darstellt, sondern vielmehr als geographischer Orientierungspunkt verwendet wurde. In anderen Werken ist von so genannten Wortfeldern die Rede, die typisch für die Ära Adenauer sind:

In den 50er und frühen 60er Jahren, der sog. ,Ära Adenauer' dominierte ein Wortfeld, das keineswegs nur von CDU-Politikern benutzt wurde, das aber weitgehend von der CDU geprägt wurde und in dem sich diejenigen Einstellungen und Konzeptionen, diejenigen politischen Schwerpunkte und Entwicklungen spiegeln, die vor allem mit der Politik der Union, insbesondere ihrer führenden Repräsentanten Adenauer und Erhard, identifiziert wurden. ${ }^{366}$

Nachfolgend werden unter anderen auch die von Kurt Schumacher verwendeten Ausdrücke Ostzone und Wohlstand angeführt, was als weiteres Indiz für die übliche Verwendung dieser Ausdrücke in den 1950er Jahren angesehen werden kann. Der letztgenannte Ausdruck ist in den untersuchten Parteiprogrammen nachweisbar, wie weiterhin Ausdrücke, die als ökonomische Sachverhalte hohe Relevanz besitzen, wie persönlich materielle Bedürfnisse (Lebensstandard, Beseitigung der Wohnungsnot, sozialer Wohnungsbau oder Lastenausgleich), makro-ökonomische Ordnungsprinzipien (Soziale Marktwirtschaft, stabile Währung oder Partnerschaft), Prozesse (Wirtschaftswachstum) sowie für die Teilung im Rahmen

364 Wengeler, Martin: „Die Deutschen Fragen. Leitvokabeln der Deutschlandpolitik." In: Politische Leitvokabeln in der Adenauer-Ära. Hg. v. Karin Böke/Frank Liedtke/Martin Wengeler. Berlin/New York 1996b, S. 369/370

365 Schumacher, Kurt: „Die Sozialdemokratie und die Verteidigung Deutschlands." Gehalten am 23.08.1950 in Bonn. In: Kurt Schumacher. Reden - Schriften - Korrespondenzen 1945-1952. Hg. v. Willy Albrecht. Berlin/Bonn 1985, S. 828-835

366 Klein 1989, S. 33 
des Ost-West-Konlikts charakteristische Ausdrücke (Freiheit, Bolschewismus, Kommunismus, Atlantisches Bündnis).

Schumacher verwendet in seiner Rede die Ausdrücke Leistung und sozialer Wohlstand, die ebenfalls in einigen Parteiprogrammen nachgewiesen werden können. Laut WENGELER (1996c) war das letztgenannte ein „weiteres wichtiges Schlagwort zur Vermittlung der Ziele der ,sozialen Marktwirtschaft'. Der Ausdruck wurde aber erst nach vollzogenem Wirtschaftsaufschwung in der zweiten Hälfte der 50er Jahre zur politischen Werbung genutzt. “367 In allen untersuchten Parteiprogrammen kann der Ausdruck Wohlstand erstmals im Godesberger Programm von 1959 nachgewiesen werden. Insofern nimmt Schumacher mit der Verwendung des Ausdrucks Wohlstand in seiner Rede das politische Geschehen und deren Inhalte um 1950 vorweg und geht dem Zeitgeist voraus. Es ist daher anzunehmen, dass der von ihm verwendete Ausdruck Ostzone ebenfalls im Jahre 1950 Teil des damaligen aktiven Sprachgebrauchs war.

Veraltet erscheinen nach heutigem Ermessen auch Ausdrücke, die in der Realität keine Entsprechung mehr zu haben scheinen, da sie bestimmte Organisationsprinzipien oder Sachverhalte bezeichnen, die nicht mehr existieren. Dazu gehören die Ausdrücke Feudalbarone, Junker und Junkertum oder Soldatenräte.

Eine besondere Betrachtung verdient der Ausdruck Baal im Parteiprogramm der KPD von 1918/19. Es kann an dieser Stelle festgehalten werden, dass der Ausdruck abwertend gebraucht wird und sinngemäß mit dem heutigen Ausdruck Götze gleichgesetzt werden kann. Als altertümlich erscheint der Ausdruck Baal in nachfolgend zitierter Textstelle deshalb, weil er im heutigen Sprachgebrauch ungebräuchlich ist und selten vorkommt. Dieser Eindruck resultiert allerdings aus der heutigen Sprachbetragung, zum Zeitpunkt der Programmformulierung war der Ausdruck Baal durchaus geläufig:

(a) Das internationale Kapital - das ist der unersättliche Baal, dem Millionen auf Millionen dampfender Menschenopfer in den blutigen Rachen geworfen werden. (KPD 1918/19, S. 107)

Als ein Beispiel für den gegensätzlichen Bereich der Wortneubildung kann der Ausdruck Hausfrauisierung (DIE FRAUEN 2002, S. 7) betrachtet werden. Der Bereich möglicher Neologismen kann in dieser Untersuchung nicht ausführlich erfolgen, da die nachfolgenden detaillierten Betrachtungen der Schlagwörter und Metaphern dieser Untersuchung der

367 Wengeler, Martin: „Vom Jedermann-Programm bis zur Vollbeschäftigung. Wirtschaftspolitische Leitvokabeln." In: Politische Leitvokabeln in der Adenauer-Ära. Hg. v. Karin Böke/Frank Liedtke/Martin Wengeler. Berlin/New York 1996c. S. 423 
Parteiprogramme und der politischen Sprachverwendung zweckdienlicher sind. Die Originalität als ein wichtiger Bedingungsfaktor von Wortschöpfungsprozessen spielt bei den Parteiprogrammen eine - wenn überhaupt - marginale Rolle. Mögliche Wortneubildungen werden im nachfolgenden Kapitel Lehnwörter erneut aufgegriffen.

\subsubsection{Fremdwörter}

Fremdwort als metasprachliches Prädikat eines Teilwortschatzes, als (quasi)linguistische Kategorie meint [...] eine lexikalische (meist lexematische) Entlehnung aus nichtdeutschen Herkunftssprachen ins Deutsche als Empfängersprache. ${ }^{368}$

Die oben zitierte Definition hat demnach mit Entlehnung zu tun, die Fremdheit bestimmter Ausdrücke resultiert folglich aus der Entlehntheit und ist sprachhistorisch zu begründen. Eine solche diachrone Sprachbetrachtung lässt jedoch die Tatsache unberücksichtigt, dass Sprache und deren Elemente Wandlungen unterliegen, wie beispielsweise Assimilationsprozesse in der Schreibweise. Aufgrund dieser Problematik reformuliert LINK (1983) die oben zitierte Definition folgendermaßen:

Ein Fremdwort ist ein Wort, das aus einer anderen Sprache ins Deutsche übernommen wurde, oder ein (jüngeres) im Deutschen unter Wiederverwendung des Signifikanten einer Wortentlehnung entwickeltes Homonym einer Wortentlehnung. ${ }^{369}$

Fremdwörter können daher als Verwendungen von in den deutschen Wortschatz integrierten Entlehnungen betrachtet werden. Die untersuchten Parteiprogramme beinhalten Fremdwörter, von denen allerdings ein Großteil durchaus gängig ist und daher im jeweiligen Zeitraum der einzelnen Programme bereits im aktiven Sprachgebrauch weiter Teile der Bevölkerung etabliert gewesen sein dürfte. Die folgenden Fremdwörter der Parteiprogramme (auch als Komponenten in Komposita) sind heutzutage auch außerhalb der politischer Sprachverwendung in ihrem Gebrauch üblich und verständlich, allerdings kann hier nur eine Auswahl wiedergegeben werden: Profit, alternativ, anonym, Konflikt, Terror, Terrorismus oder Terrorist, fossil, Zertifikat, Patriarchat und patriarchalisch, archaisch, Kooperation, Mobilität und mobil, Territorien, Diskriminierung, Antisemitismus, Diskurs, Dynamik, Zivilisation, Kontroverse oder Debatte, Korruption, korrupt und korrumpieren, korrespondieren, Tourismus, Monopol, Naturreservate, Humanismus, Investition, Export und Import, orientieren, boykottieren, Motiv, Konsequenz, Kapitulant, Zynismus, Zentralisierung, mul-

368 Link, Elisabeth: „Fremdwörter - Der Deutschen liebste schwere Wörter?“ In: Deutsche Sprache. Zeitschrift für Theorie, Praxis und Dokumentation. Berlin 1983, S. 54

369 Link 1983, S. 57 
tinational, gigantisch, Konzentration, Funktion, Qualifikation und qualifiziert, Fundament, intellektuell, Varianten, objektiv und subjektiv, asymmetrisch, Reform, defensiv und offensiv, offiziell, spezifisch oder geschlechtsspezifisch, Idealismus, Ideale und ideal, Manipulation, Transparenz, Dokumentation und dokumentieren, radikal, Elite, imponierend, international, Emanzipation, Stabilität und stabil, Periode oder Legislaturperiode, Remilitarisierung, Potenzial (Bildungs-, Zukunfts- oder Entwicklungspotenzial) und potenziell, Taktik, Strategie, enorm, Demokratie, Diskussion, materiell und immateriell, Egoismus und egoistisch, Stadium, Interesse, Prinzipien, absolut, Isolation, Isolierung und isolieren, Talent, Korrekturen, korrigierbar, korrigierend, Substanz und substanziell, Metropole, Disziplin, Pazifisten, Utopie, illegal, naiv, Privileg, Kriterium, Produktion, Identität, Charakter oder Schutzcharakter.

In den Parteiprogrammen lassen sich jedoch auch eine Reihe von Fremdwörtern nachweisen, die weniger geläufig sein dürften, wie beispielsweise: Aggressor, Propaganda, propagieren oder propagandistisch, Statut, partiell, Basierung, Formation, Delegation, Delegierte oder delegieren, Alliierte, Verdikt, Annullierung, Konsumtionsfonds, Souveränität, okkupieren, Liberalisierung, protektionistisch, usurpieren, Disposition, Konfrontation, egalitär, Konfiskation, Korrelation, Integrität und Subsidiarität sowie als Komposita Korrelationsaussagen und Subsidiaritätsprinzip, Dividenden, Ämterpatronage, Konföderation oder Annexion. Diese Fremdwörter unterscheiden sich entweder hinsichtlich der Frequenz ihrer Verwendung oder hinsichtlich des Kontextes zur oben genannten ersten Kategorie von Fremdwörtern. In allgemeinen Sprachzusammenhängen wird eine Sprachgemeinschaft das Wort Konsequenz häufiger verwenden als die Wörter Konföderation oder Delegation, vorausgesetzt, die Diskussion oder die Korrespondenz handelt nicht von politischen Sachverhalten.

Die Verwendung von Fremdwörtern in den Parteiprogrammen liegt in den jeweiligen thematischen Sachverhalten und in der damit einhergehenden Verwendung von Termini eines Fachwortschatzes begründet; Termini wie Pränataldiagnostik, Diagnose, Symptom oder Organ werden zwangsläufig gebraucht, wenn der thematische Schwerpunkt im medizinischen Bereich liegt, d.h. konkret, wenn die Parteien in ihren Programmen ihre Vorstellung von Gesundheitspolitik darlegen.

Da das Grundsatzprogramm primär die Parteibasis und deren Mitglieder und nicht die Massenöffentlichkeit als Adressatengruppe anvisiert, ist eine mögliche Unverständlichkeit aufgrund der Verwendung von Fremdwörtern nebensächlich. Da jedoch insbesondere die Inhalte der Wahlprogramme auch von den Medien aufgegriffen werden (in den 1950er Jahren Zeitungen und Rundfunk, zunehmend seit den 1960er Jahren und heute neben den Printmedien auch Fernsehen und Internet) und sich somit an die breite Schicht der Bevölkerung richtet, sollte ein Wahlprogramm nicht mit Fremdwörtern überladen sein, nachweisen 
lässt sich eine geringere Häufigkeit von Fremdwörtern in den Wahlprogrammen jedoch nicht. Die letztgenannte Adressatengruppe könnte dann auch die Ursache für die Verwendung der Wörter sein, die als geläufige und etablierte Fremdwörter oben angeführt wurden. Es ist aber anzunehmen, dass auch heutzutage die breite Schicht der Bevölkerung ohne ausgeprägtes Interesse am politischen Geschehen die Verwendung der Wörter Konföderation oder Unilateralismus allenfalls im Kontext nachvollziehen kann.

Viele der in diesem Kapitel zitierten und vermeintlich als Fremdwörter eingestuften Ausdrücke sind jedoch weder Wortentlehnungen noch Homonyme von solchen, sondern resultieren innerhalb des Deutschen aus Morphemkombinationen, sie sind daher Komposita aufgrund von Wortbildungsprozessen. Entlehnt sind jedoch die einzelnen Komponenten oder Konstituenten der Komposita, daher lassen sich diese Komposita auch als Lehnwortbildungen bezeichnen. Die Ausdrücke Leninismus und Marxismus stellen sich beispielsweise dar als Ableitungen mit dem Namen eines Deutschen (Marx) und eines Russen (Lenin) als einer Konstituente und einem entlehnten Affix (-ismus) als der anderen Konstituente. Die Bezeichnung Regulierung stellt sich dar als Ableitung mit einer entlehnten Konstituente als Stamm (Regul- von lat. regulare) und einer indigenen Konstituente als Affix (-ierung), der Ausdruck Nazi ist ein abgeleitetes Kurzwort von Nationalsozialist als Fremdwort. Die Ausdrücke Gewalt- und Wirtschaftskriminalität, Bürgerdialog, Lebens- und Entfaltungschancen sind Komposita mit einer indigenen Komponente als Bestimmungsglied (Gewalt, Wirtschaft, Bürger, Leben und Entfaltung) und einer entlehnten Konstituente als Grundwort (Kriminalität, Dialog und Chance), und die Wörter Parteiprogramm und Herrschaftsmechanismus sind als Komposita mit ausschließlich im Deutschen als Simplizia disponiblen Konstituenten $\mathrm{zu}$ verstehen, wobei Parteiprogramm im Gegensatz zu Herrschaftsmechanismus aus ausschließlich entlehnten Konstituenten zusammengesetzt ist. Unter Berücksichtigung dieser Ausführung ergibt sich abschließend folgende Definition für Fremdwörter:

Fremdwörter sind Wörter, bei denen zumindest eine ihrer ausdrucksseitig als solche in Erscheinung tretenden, ggf. (im Deutschen) kombinierten, morphemischen Konstituenten bzw. ein (älteres) im Deutschen unter Wiederverwendung des Signifikanten zu der fraglichen Konstituente weiterentwickeltes Homonym einer Konstituente sowie ggf. eventuell auch die Weise der Kombination der Konstituenten $\mathrm{zu}$ irgendeinem näheren oder ferneren Zeitpunkt der Geschichte des Deutschen aus einer beliebigen Fremdsprache übernommen wurde. ${ }^{370}$

370 Link 1983, S. 60 


\subsubsection{Lehnwörter}

Im weiteren Sinne fallen auch etablierte Entlehnungen wie Nonsens oder Korps in den Bereich der Fremdwörter. Im Folgenden soll nun eine tiefergehende Betrachtung ausgewählter Lehnwörter erfolgen. In den analysierten Parteiprogrammen können das Substantiv Manager als Anglizismus sowie weiterhin die Ausdrücke Nationalbudget und Reglement nachgewiesen werden; in den anderen Parteiprogrammen finden sich außerdem die Ausdrücke Team, Trend, City, Human Development Report, Homepage, Internet, Konsumtionsfonds, Avantgarde, Bourgeoisie, Engagement und engagiert, Etage, Zivilcourage, Steuerdumping, Gender Mainstreaming, Global Restructuring, Ehegattensplitting sowie Marketing, Sportangebot, Fairness und fair:

(50) (a) In der Großwirtschaft ist die Verfügungsgewalt überwiegend Managern zugefallen, die ihrerseits anonymen Mächten dienen. (Godesberger Programm 1959, S. 76)

(b) Eine solche Politik bedarf der volkswirtschaftlichen Gesamtrechnung und des Nationalbudgets. Das Nationalbudget wird vom Parlament beschlossen. (Godesberger Programm 1959, S. 74)

(c) Die künstlerische Entfaltung darf durch kein Reglement, insbesondere durch keine Zensur, beschränkt werden. (Godesberger Programm 1959, S. 83)

(d) [...] Die Möglichkeiten von Teamarbeit und Delegation von Verantwortung sind zu nutzen. (CDU 1971, S. 83)

(e) Steuerdumping erzeugt unfaire Wettbewerbsbedingungen. (B 90/GRÜNE 2002, S. 50)

(f) Gender Mainstreaming formuliert geschlechterdemokratische Ansprüche an Arbeit. (B 90/GRÜNE 2002, S. 68)

(g) Der ,Schmierstoff' des Global Restructuring ist die moderne Hochtechnologie und die Ideologie von der Informationsgesellschaft ohne Grenzen. [...] Wir wollen das Ehegattensplitting im Einkommenssteuerrecht streichen. [...] Mit dem Wegfall des Ehegattensplittings würde die frauendiskriminierende Lohnsteuerklasse $\mathrm{V}$ entfallen, [...]. (DIE FRAUEN 2002, S. 7/18)

Die Verwendung des im heutigen Sprachgebrauch etablierten Ausdrucks Manager wirft die Frage auf, ob dieser bei der Verwendung im Godesberger Programm im Jahre 1959 bereits etabliert gewesen ist oder die SPD damit ihrer Zeit voraus war. Im Folgenden soll nun am Beispiel des Managers ein ausführlicher Versuch unternommen werden, diese Frage zu klären. Dem Ausdruck Manager in Äußerung (a) können drei Bedeutungen zugeschrieben werden: 
1. „Person, die die beruflichen und geschäftlichen Interessen von Personen oder Gruppen im Showgeschäft bzw. Personen oder Mannschaften im Berufssport vertritt"; $[\ldots]$

2. „Person, die im politischen Bereich in leitender Funktion eine Sache organisiert und durchführt, mit Geschick handhabt, bewältigt und eine gewünschte Konstellation zustande bringt"; $[\ldots]$

3. „Person, die ein Wirtschaftsunternehmen mit weitgehender Verfügungsgewalt und Entscheidungsbefugnis leitet." ${ }^{\text {"371 }}$

In Äußerung (a) des Godesberger Programms ist der Ausdruck im wirtschaftlichen Kontext der dritten Definition folgend verwendet. Für die Verwendung der Bezeichnung Manager für eine Person nach der 3. Definition (Berufsbezeichnung) kann das Jahr 1949 als Erstbeleg im Paderborner Korpus datiert werden; 1915 wurde der Ausdruck in dieser Bedeutung erstmalig in einem deutschen Wörterbuch (Tesch) verbucht. Die Verwendung des Ausdrucks in der Bedeutung der ersten Definition geht als Erstbeleg im Paderborner Korpus auf das Jahr 1909 und als Erstbuchung in einem deutschen Wörterbuch (DUDEN-Rechtschreibung, Mannheim) auf das Jahr 1941 zurück. Folglich ist die Verwendung des Ausdrucks Manager im Godesberger Programm im Jahre 1959 keine Wortneuschöpfung der SPD, sondern vielmehr eine übliche Vokabel des gesellschaftspolitischen und wirtschaftlichen Bereichs; formal ist der Ausdruck Manager als Anglizismus erkennbar. Wie oben erwähnt stellen die drei Definitionen jedoch nicht drei unterschiedliche Bedeutungen dar, sondern es handelt sich vielmehr um eine Bedeutung, die jeweils auf die drei verschiedenen Bereiche Sport (1.), Politik (2.) und Wirtschaft (3.) angewendet wird. Die SPD verwendet den Ausdruck Unternehmensmanager auch in ihrem Programm von 1989.

Die Ausdrücke Reglement und Nationalbudget des Godesberger Programms stellen weitere Entlehnungen dar. Bei der Betrachtung der Ausdrücke Teamarbeit, Steuerdumping oder Ehegattensplitting in den Äußerungen (d), (e) und (g) fällt auf, dass die Entlehnungen als Komponenten (Glieder) von Komposita verwendet werden. So schreibt das AnglizismenWörterbuch, dass Dumping "meist in Komposita" gebraucht wird zur Bezeichnung einer "Maßnahme zur Förderung des Außenhandelsabsatzes, bei der Waren oder Dienstleistungen unter dem Markt- oder Inlandspreis angeboten werden, um die Konkurrenz zu unterbieten und so ausländische Märkte zu gewinnen." ${ }^{372}$ Erstmalig in einem deutschen Wörterbuch (DUDEN-Rechtschreibung, Mannheim) erscheint dieser Anglizismus im Jahre 1929.

371 Anglizismen-Wörterbuch 1993, S. 873/874

372 Anglizismen-Wörterbuch 1993, S. 406 
Der Ausdruck Gender Mainstreaming, der in den Parteiprogrammen der PDS (2003) und der GRÜNEN (2002) gebraucht wird, verdient in diesem Zusammenhang eine besondere Erwähnung. Der Ausdruck ist in deutschen und englischen Wörterbüchern (DUDEN 1999, Brockhaus/Wahrig 1982, OXFORD 2000373, Langenscheidt 1996374) nicht gelistet. Daher kann vermutet werden, dass es sich bei diesem Ausdruck um eine im deutschen Sprachgebrauch noch nicht etablierte Bezeichnung handelt, jedoch spricht die Verwendung des Wortes in einem Parteiprogramm (aufgrund der Adressatengruppe und der gattungsspezifischen Funktionen) gegen die These, dass die Ausdrücke noch nicht etabliert sind, da die Originalität oder die Wortschöpfung bei der Sprachverwendung in einem Parteiprogramm lediglich eine untergeordnete Rolle spielt. Vielmehr mag die Vermutung plausibel erscheinen, dass der Ausdruck in innerparteilichen Debatten verwendet und somit in den Parteiprogrammen schriftlich fixiert wurde. Diese Tatsache wiederum würde für die Textsorte Parteiprogramm bedeuten, dass die Sprachverwendung als Wirklichkeitsabbildung zu bewerten wäre. Die PDS versteht unter der Bezeichnung Gender Mainstreaming den Sachverhalt der Geschlechtergerechtigkeit. Der Ausdruck mainstream ist dagegen im Anglizismen-Wörterbuch angeführt, mit der Bedeutung: „Form des Jazz und der Popmusik, die die Hauptrichtung der Entwicklung darstellt und zwischen den extremen Richtungen des Traditionellen und Experimentellen liegt, jedoch keiner Stilrichtung eindeutig zuzuordnen ist ${ }^{\prime \prime 375}$ sowie im Langenscheidt als "Hauptströmung “376; allerdings verwenden die beiden Parteien den Ausdruck als ing-Konstruktion. Da die Wendung Gender Mainstreaming in dieser Form auch in fremdsprachlichen Nachschlagewerken nicht gelistet ist, liegt die Vermutung einer ad-hoc-Bildung nahe, die aus einem Prozess der Übergeneralisierung resultiert (hier das Anhängen von -ing an Wortformen); für diese Betrachtung spricht auch die Verwendung von Majuskeln an den Wortanfängen (in englischen dictionaries als mainstream).

Weiterhin fällt auf, dass diese Anglizismen in den jüngeren Parteiprogrammen nachgewiesen werden können. Diese Beobachtung ist teilweise begründbar: Beispielsweise bestand in der Zeit vor der privaten Nutzung des Internets keine Notwendigkeit für eine Sprachgemeinschaft, einen Ausdruck wie Homepage zu verwenden, da kein Referenzobjekt existierte, das bezeichnet werden musste. Dies änderte sich Anfang der 1990er Jahre mit der beginnenden flächendeckenden Nutzung des World

373 OXFORD Advanced Learner's Dictionary of Current English. Hg. v. Sally Wehmeier. Oxford ${ }^{6} 2000$.

374 Langenscheidts Handwörterbuch Englisch. Berlin/München ${ }^{2} 1996$.

375 Anglizismen-Wörterbuch 1993, S. 866

376 Langenscheidt 1996, S. 393 
Wide Web. Da die Programmiersprachen überwiegend englisch sind, begannen sich mit zunehmender Verwendung des Internets auch die Ausdrücke Homepage oder Internet zu etablieren und werden heutzutage kaum mehr als Bezeichnungen mit fremdsprachlicher Herkunft betrachtet (zumindest nicht von Gesellschaftsmitgliedern, die das Internet nutzen und fremdsprachliche Kenntnisse besitzen). Die Komponenten Team im Ausdruck Teamarbeit sowie fair in unfair oder Fairness sind aufgrund ihrer längeren Verwendungsdauer im deutschen Sprachgebrauch etabliert und wenig spektakulär:

Generell gilt, daß die fremdsprachliche Herkunft mit zunehmender Dauer der Verwendungsgeschichte immer unauffälliger wird. Ausdrücke wie Teamwork, Hitparade oder Spray z.B. sind im heutigen deutschen Wortschatz alle relativ unauffällig, das gilt vollends für Wörter mit etwas längerer Gebrauchsgeschichte wie Sport. 377

\subsubsection{Metaphern}

Metapher heißt Übertragung: eine Wortbedeutung wird in einem ihr von Hause aus nicht zukommenden Sinne verwendet. ${ }^{378}$

Die oben zitierte knappe Definition soll nachfolgend ausführlicher betrachtet und auf die Sprachverwendung im Godesberger Programm und in den anderen Programmen bezogen werden.

Geht man von der Annahme aus, dass das Denken allgemein metaphorisch ist, dann ist auch die Sprache, verstanden als eine Form der Realisierung von Gedachtem, von Metaphern durchsetzt. So entsprechen Metaphern den grundlegenden Denkmodellen und eröffnen als sprachliche Phänomene die Möglichkeit, die Interaktion von Kommunikation, Kognition und sprachlichen Strukturen aufzuzeichnen und zu erklären; „sie stellen Modelle bereit, die auf Grundformen unserer Wahrnehmung und unserer Lebensweise beruhen und uns Erscheinungen und Sachverhalte mental verfügbar machen. “379 Die Metapher legt dabei „das Wahrnehmungsmuster fest, auf das die Menschen reagieren. “380

Die Verschiebung bestimmter Wahrnehmungsmuster auf artfremde Bereiche scheint charakteristisch nicht nur für das Godesberger Programm zu sein:

377 Gloning 2003, S. 69

378 Kayser 1992, S. 123

379 Schäffner, Christina: „Europapolitische Metaphorik in England und Deutschland." In: Sprachstrategien und Dialogblockaden. Linguistische und politikwissenschaftliche Studien zur politischen Kommunikation. Hg. v. Josef Klein/Hajo Dieckmannshenke. Berlin/New York 1996, S. 153

380 Edelman 1976, S. 148 
(51) (a) Die Bändigung der Macht der Großwirtschaft ist darum zentrale Aufgabe einer freiheitlichen Wirtschaftspolitik. Staat und Gesellschaft dürfen nicht zur Beute mächtiger Interessengruppen werden. (Godesberger Programm 1959, S. 75)

(b) Wo mit anderen Mitteln eine gesunde Ordnung der wirtschaftlichen Machtverhältnisse nicht gewährleistet werden kann, ist Gemeineigentum zweckmäßig und notwendig. (Godesberger Programm 1959, S. 76)

(c) Freie Gemeinden sind unerläßlich für eine lebendige Demokratie. (Godesberger Programm 1959, S. 72)

Der Arbeitnehmer wird in Äußerung (a) als Beute dargestellt. Somit wird in (a) ein für das Tierreich typisches und gebräuchliches Wahrnehmungsmuster auf den Bereich der Gesellschaft und der Arbeitswelt übertragen, was durch die Verwendung des Ausdrucks Bändigung weiterhin betont wird. Zwar ist beispielsweise bei Naturgewalten ebenso von Bändigung die Rede. Indem jedoch in (a) die freiheitliche Wirtschaftspolitik sinngemäß die Rolle des Dompteurs übernimmt wird der Großwirtschaft mit ihrer Macht unweigerlich die Rolle des unberechenbaren wilden und daher gefährlichen Raubtieres, das es zu zähmen (zu bändigen) gilt, zugeschoben.

Der Ausdruck gesunde Ordnung (b) wurde bereits als Leerformel besprochen; die Definition des metaphorischen Sprachgebrauchs lässt sich hier ebenso anwenden, da durch die Verwendung der Adjektive gesund sowie lebendig in Äußerung (c) das gebrauchsübliche Wahrnehmungsmuster des körperbezogenen Belebtheitszustands auf den gebrauchsunüblichen gesellschaftspolitischen Bereich verschoben wurde. Das Attribut gesund wird hier in der Bedeutung von ausgewogen, funktionierend oder gerecht verteilt gebraucht; durch die Verwendung des Attributs lebendig wird veranschaulicht, dass der abstrakte Ausdruck der Demokratie tatsächlich gelebt, d.h. realisiert werden kann. Die Verwendung des Adjektivs gesund ist auch in Verbindung mit anderen Substantiven nachweisbar: So treten die Parteien für die Herstellung gesunder internationaler Beziehungen (SED 1963, S. 252) ein, sprechen von gesunden, vernünftigen Bedürfnissen des allseitig entwickelten Menschen (SED 1963, S. 332), von der Schaffung eines gesunden Mittelstandes (NSDAP 1920, S. 16), von der Sicherung einer gesunden Umwelt (FDP 1971, S. 273) und von einer starken und gesunden Wirtschaft (CSU 1968, S. 238) sowie vom Erhalt und der Festigung gesunder Betriebsstrukturen (PDS 2003, S. 19). Die Bedeutungen der Ausdrücke gesund, stark und lebendig bleiben nicht unverstanden, sondern dienen vielmehr der Veranschaulichung und unterstreichen die Brisanz des Inhalts, da der Rezipient durch den Kontext vorbereitet ist und die Metapher, d.h. die auf einen gebrauchsunüblichen Kontext übertragene (projizierte) Wortbedeutung, sofort verstehen kann. Dieses Phänomen 
liegt auch der etablierten und vielfach zitierten Definition von LAKOFF/JOHNSON (1980) zugrunde: „The essence of metaphor is understanding and experiencing one kind of thing in terms of another." ${ }^{381}$

Die oben zitierten Verwendungsweisen von gesund, stark und lebendig stellen die positive Erscheinungsform des metaphorischen Grundmusters „KRISEN SIND KRANKHEITEN" dar; diese ist als „medizinische und Krankheitsmetaphorik [...] heute überwiegend in bezug auf die Wirtschaft gebräuchlich.“382 Diesem Bereich der Gesundheits- oder Krankheits-Metaphorik gehört auch der Ausdruck Belebung des Föderalismus (B 90/GRUNE 2002, S. 115) an. Eine negative Erscheinungsform dieses Metaphernkonzepts lässt sich mit dem Ausdruck parasitär und Parasitismus in den Äußerungen (d) und 56 (a) nachweisen; eine Krankheits-Metapher liegt dann vor, wenn „politische Gegner oder ethnische Gruppen als Krankheiten (Pest, Cholera, Krebs, Syphilis, Geschwür) oder als deren Erreger bzw. Überträger (Ungeziefer, Parasiten, Bazillen, Ratten, Schmeiffliegen) metaphorisiert werden. “383

(d) Der Imperialismus ist - wie W. I. Lenin feststellte - monopolistischer und parasitärer oder faulender Kapitalismus, er ist sterbender Kapitalismus. [...] So wurde in Westdeutschland die längst zum Abtreten reife parasitäre kapitalistische Gesellschaftsordnung durch Betrug, Gewalt und Bestechung künstlich am Leben erhalten, zum Schaden des deutschen Volkes und vor allem der Bevölkerung Westdeutschlands. (SED 1963, S. 215/334)

BURKHARDT (2003) sieht in der Verwendung solcher Bezeichnungen gleichzeitig eine implizite Anstiftung zur Gewalt gegen die bezeichneten Personen verwirklicht, da aufgrund der Gefährlichkeit, die von den Krankheiten sowie deren Erreger und Überträger ausgeht die Nebenbedeutung einhergeht, dass diese vernichtenswert sind und beseitigt werden dürfen oder gar müssen. Diese Verwendungsweise ist charakteristisch für die Sprachverwendung während der Zeit des NS-Regimes: „Zur Gegner-Benennung fügte man gern die biologische Metapher von den Parasiten, den Bazillen oder dem vergifteten Blut." ${ }^{384}$ Im frühen Parteiprogramm der NDSAP aus dem 1920 lassen sich jedoch (noch) keine Verwendungsweisen nachweisen, die diesem metaphorischen Muster folgen.

In Übereinstimmung mit LAKOFF/JOHNSON (1980), die den Sinn von Metaphern im Hervorheben (highlighting) und Ausblenden (hiding) sehen,

381 Lakoff, George/Johnson, Mark: Metaphors we live by. Chicago/London 1980, S. 5

382 Burkhardt 2003, S. 371/372

383 Burkhardt 2003, S. 378

384 Bachem 1979, S. 128 
scheinen Metaphern somit als Erscheinungsform sprachlicher Handlungen dazu zu dienen, selektive Wahrnehmungen zu ermöglichen, zu intensivieren und andere wiederum zu ignorieren. Metaphern strukturieren damit die Darstellung und Wahrnehmung von Ereignissen.

Wie oben bereits geschehen lassen sich viele der in den verschiedenen Parteiprogrammen verwendeten Metaphern unterschiedlichen Kategorien zuordnen. Mit der umfangreichen Kategorie der Kampf-Metaphern soll die Analyse der verwendeten Metaphern beginnen und andere Bereiche wie die "Bau- und Wege-Metaphern“385 oder die Schiffs-Metaphern anschließend exemplarisch beleuchtet werden. Dabei umfassen die nachfolgend dargestellten Kategorien häufig verwendete Ausdrücke und werden deshalb im Gegensatz zu den biologischen Metaphern oder den Krankheits- und Gesundheits-Metaphern gesondert hervorgestellt.

\subsection{Kampf-Metaphern}

Diese oben formulierte Annahme kann durch die nachfolgenden Textbeispiele veranschaulicht werden:

(52) (a) In der heutigen Wirtschaft sind die Arbeitnehmer denen ausgeliefert, die die Kommandostellen der Unternehmen und ihrer Verbände besetzen, wenn sie ihnen nicht in unabhängigen Gewerkschaften ihre solidarische, demokratisch geordnete Kraft entgegenstellen, um die Arbeitsbedingungen frei vereinbaren zu können. (Godesberger Programm 1959, S. 78)

(b) Die Arbeiterschaft war in ihrem Kampf nur auf sich gestellt. Ihr Selbstbewußtsein wurde geweckt durch die Erkenntnis ihrer eigenen Lage, durch den entschlossenen Willen, sie zu verändern, durch die Solidarität in ihren Aktionen und durch die sichtbaren Erfolge ihres $\underline{\text { Kampfes. }}$.

(c) Schweren Rückschlägen und manchen Irrtümern zum Trotz hat die Arbeiterbewegung im neunzehnten und zwanzigsten Jahrhundert die Anerkennung vieler ihrer Forderungen erzwungen. Der einst schutz- und rechtlose Proletarier, der sich für einen Hungerlohn täglich sechzehn Stunden schinden mußte, erreichte den gesetzlichen Achtstundentag, den Arbeitsschutz, die Versicherung gegen Arbeitslosigkeit, Krankheit, Siechtum und für seinen Lebensabend. Er erreichte das Verbot der Kinderarbeit, der Nachtarbeit für die Frauen, den Jugend- und Mutterschutz und bezahlten Urlaub. Er erstritt sich die Versammlungsfreiheit, das Recht zum gewerkschaftlichen Zusammenschluß, das Tarifrecht und das Streikrecht. Er ist dabei, sein Recht auf Mitbestimmung durchzusetzen. [...]

(d) Diese Erfolge sind Meilensteine auf dem opferreichen Weg der Arbeiterbewegung. (Godesberger Programm 1959, S. 85/86) 
(e) Solidarität ist zugleich Waffe der Schwachen im Kampf um ihr Recht und Konsequenz aus der Einsicht, daß der Mensch der Mitmenschen bedarf. (SPD 1989, S. 13)

(f) Die Imperialisten fürchten den weiteren Vormarsch der fortschrittlichen Kräfte. (DKP 1969, S. 312)

(g) Mit den hohen sozialen Sicherheitsrisiken und Störanfälligkeiten der Atomanlagen wird der Marsch in den Atomstaat gerechtfertigt. [...] Wir sind uns bewusst, dass demokratische Freiheiten und Menschenrechte nur dann eine Zukunft haben, wenn wir uns rechtzeitig gegen die Gigantomanie zentralistischer Energie Versorgungsmonopole wehren und den Marsch in den totalitären Atomstaat vermeiden. (GRÜNE 1980, Kap. II, 6)

(k) Abhängig von Banken, Rohstoffbörsen, Konzernen und Staaten des Nordens, [...], zum Manövrierfeld des Ost-West-Konflikts erniedrigt, oft von korrupten Eliten ausgebeutet, ringt der Süden um seine Zukunftschance. (SPD 1989, S. 14)

(1) Je fester fundiert und je besser organisiert die sozialistische Weltwirtschaft und der sozialistische Weltmarkt sind, um so besser wird es möglich sein, die nationalen Wirtschaften der sozialistischen Länder und den Wohlstand ihrer Völker maximal zu entwickeln und alle Störmanöver des Weltimperialismus abzuweisen. (SED 1963, S. 321)

(m) Es wäre verkehrt, die Kraft des Imperialismus und seine Fähigkeit zu unterschätzen, an bestimmten Abschnitten der ökonomischen Kampffront aktiv anzugreifen. [...] Der Aufruf der Kommunistischen Partei Deutschlands bildete die Grundlage für die Einheitsfront zwischen der Kommunistischen Partei Deutschlands und der Sozialdemokratischen Partei Deutschlands. [...] Das kann sie nur, wenn die Einheitsfront der sozialdemokratischen, kommunistischen, christlichen und parteilosen Arbeiter verwirklicht wird und die Arbeiterklasse in organisierter, politisch wirkungsvoller Weise auftritt. Die Einheitsfront der westdeutschen Arbeiter entsteht im Kampf gegen die atomare Aufrüstung und für die Sicherung des Friedens. (SED 1963, S. 218/227/247)

Die unterstrichenen Ausdrücke der Äußerungen (a) bis (m) stellen eine metaphorische Verwendungsweise insofern dar, als dass hier ein Wahrnehmungsmuster festgelegt ist, auf das der Rezipient reagiert. Dabei handelt es sich um das Wahrnehmungsmuster des Kampfes, das als Spezialfall dem Grundmuster „RATIONAL (POLITICAL) ARGUMENT IS WAR" folgt, von dem LAKOFF/JOHNSON (1980) sagen: "This metaphor allows us to conceptualize what a rational argument is in terms of something that we understand more readily, namely, physical conflict. “386

386 Lakoff/Johnson 1980, S. 61 
Das metaphorische Grundmuster ist somit charakteristisch für die Beschreibung alltäglicher politischer Auseinandersetzungen zwischen verschiedenen Parteien im innenpolitischen Diskurs oder zur Beschreibung langwieriger Durchsetzungsprozesse. Als Wahrnehmungsmuster wird es dann auf einen gebrauchsunüblichen Bereich (z.B. Verbesserung der Arbeitsbedingungen) übertragen:
Metaphern ziehen Verschiebungen in der Kollokation auf der lexikali- schen Ebene unter spezifischen semantischen Bedingungen nach sich, jedoch ohne Rücksicht auf etwaige andere kontextabhängige Be- schränkungen stilistischer Natur. ${ }^{387}$

Die metaphorische Verwendung des Ausdrucks Kampf für die Bemühungen zur Erreichung besserer gesellschaftlicher und politischer Bedingungen (z.B. Arbeitsbedingungen, Gleichberechtigung der Frau) ist ein häufig verwendetes Sprachhandlungsmuster, insbesondere bei den sozialistischen und kommunistischen Parteien, d.h. bei den Parteien, die ihre parteigeschichtlichen Wurzeln in der Arbeiterbewegung haben. Daher lässt sich der Ausdruck Kampf (auch als Bestandteil von Komposita wie Befreiungskampf oder Kampfgenossen) mit folgender Häufigkeit nachweisen: Eisenacher Programm 1869 (3), NSDAP 1920 (3), Godesberger Programm 1959 (2), SED 1963 (89), DKP 1969 (48), FDP 1971 (1), NPD 1973 (7) und GRÜNE 1980 (2).

Die Äußerung (a) greift mit dem Ausdruck Kommandostellen besetzen eine Vokabel des militärischen Bereichs auf und überträgt sie in den Bereich der Arbeitswelt bzw. der Unternehmensstruktur. Indem von der Besetzung einer Kommandostelle, nicht aber vom Innehaben einer Führungsstelle, einer Führungsposition oder einer leitenden Position die Rede ist, erhält die Äußerung eine kämpferische Nebenbedeutung. Der Unternehmer oder der leitende Angestellte wird als kommandierender Befehlshaber dargestellt und der Arbeitnehmer erhält sinngemäß den Status des gehorsamen Befehlsausführers ohne jegliches Mitspracherecht. Der Ausdruck Kommandostelle besetzen kann der oben dargestellten Kategorie der Kampf-Metaphern zugeordnet werden.

Allerdings muss berücksichtigt werden, dass die metaphorische Verwendung dieser Ausdrücke bei politischen Auseinandersetzungen eine lange Tradition hat und insbesondere die Ausdrücke Kampf und Rückschläge auch in der Beschreibung sportlicher Auseinandersetzungen oder bei Wettkämpfen gebräuchlich sind. Indem die SPD vom Kampf für die Arbeiterschaft spricht, so bedeutet dies, dass der Krieg als vage definiertes Mittel zur Erreichung bestimmter und erwünschter Ziele wahrgenommen wird. Dass die Arbeiterschaft ihre Ziele nicht mit kriegerischen Mitteln durchsetzt/durchgesetzt hat, wird im Hinblick auf die Aufzäh-

387 Enkvist 1972, S. 38 
lung der erreichten Ziele in Äußerung (b) deutlich (bestimmte Versicherungen, Verbote, Freiheiten, Rechte sowie bessere Bedingungen erreichen, erstreiten und durchsetzen). Das Verb erstreiten scheint hierbei einen Grenzfall darzustellen, da das Verb ein durchaus übliches sprachliches Handlungsmuster zur Erreichung bestimmter Ziele in einer Diskussion (verstanden als verbaler Kampf) beschreibt. Jedoch unterstreicht die Verwendung des Ausdruck schwere Rückschläge auch hier die Wahrnehmung des Kampfes und steigert sie zur Wahrnehmung kriegerischer Auseinandersetzungen, ebenso wie die Verwendung des Ausdrucks opferreicher Weg in Äußerung (c) und die Bezeichnung des Grundwerts Solidarität als Waffe in Äußerung (d). Ebenso sind die Ausdrücke Marsch und Vormarsch in den Äußerungen ( $f$ ) und (g) aus dem Bereich der militärischen Kampfhandlungen entlehnt. Dazu gehört die Verwendung des militärtaktischen Ausdrucks Manöver in den Äußerungen (k) und (l) sowie parlamentarische Obstruktionsmanöver der Bourgeoisie (KPD 1918/19, S. 112) ebenso wie die Komponente Front in den Komposita Einheitsfront und Kampffront der SED in Âußerung (m). Alle anderen Parteien verzichten in ihren Programmen auf die Verwendung des Ausdrucks Front, lediglich die KPD spricht an einer Stelle von der geschlossenen Front des gesamten deutschen Proletariats (KPD 1918/19, S. 112).

Das Ziel dieser Übertragung als metaphorische Verwendungsweise ist die Betonung bzw. Verstärkung der Mühen, die es die Arbeiterschaft kostet/gekostet hat, bestimmte Ziele zu erwirken (die Arbeiterschaft musste zur Durchsetzung ihrer Ziele "Opfer bringen“). Diese Ansicht bringt die SED in ihrem Programm anschaulich zum Ausdruck:

(n) Seit über hundert Jahren ist die sozialistische Gesellschaft das Kampfziel der revolutionären deutschen Arbeiterbewegung. Ihrem heroischen und opferreichen Kampf ist es zu verdanken, daß heute der Sozialismus in der Deutschen Demokratischen Republik Wirklichkeit wird. (SED 1963, S. 208)

In Bezug auf die Werbefunktion von Parteiprogrammen sind die dargestellten Übertragungen als Mittel zur Hervorhebung des Geleisteten zu verstehen (in oben zitierter Äußerung (n) sogar als Heroisierung des Geleisteten). Metaphern bieten hierfür eine kurze und prägnante Möglichkeit.

Zur Verdeutlichung sollen nachfolgend zwei Textbeispiele zitiert werden, in denen der Ausdruck Kampf als Komponente von Komposita in Bezug auf den militärischen Bereich und somit nicht metaphorisch verwendet wird:

(53) (a) Die Ausrüstung der Bundeswehr darf nicht vernachlässigt, ihre Kampfkraft und ihr Einsatzwille nicht geschwächt werden. (CDU 1971, S. 54) 
(b) Wir lehnen den Einsatz von chemischen Kampfmitteln ab. (GRÜNE 1980, Kap. V, 1.7)

\subsection{Nautische Metaphern/Schiffs-Metaphern}

(54) (a) Zu einer weltweiten Wende und Kurskorrektur zu kommen, gehört zu den größ-ten Herausforderungen und Aufgaben der Politik in den kommenden Jahren und Jahrzehnten. [...] Das Umsteuern auf einen nachhaltigen Kurs ist nicht nur ökologisch sinnvoll, es ist auch wirtschaftlich vernünftig. [...] Damit haben wir das Ruder gedreht, die Energiewende ist eingeleitet, jetzt müssen wir auf diesem Kurs Fahrt aufnehmen. (B 90/GRÜNE 2002, S. 17/27/32)

(b) Wir werden uns nicht an einer Regierung beteiligen, die den zerstörerischen Kurs fortführt. (GRÜNE 1980, Kap. I)

(c) Sozialismus ist in dieser Stunde der einzige Rettungsanker der Menschheit. (KPD 1918/19, S. 109)

Die oben zitierten Äußerungen (a) und (b) der GRÜNEN aus den Jahren 1980 und 2002 sowie Äußerung (c) der KPD sind die einzigen Textstellen in allen untersuchten Parteiprogrammen, die nautische Metaphern beinhalten. Als solche sind die unterstrichenen Ausdrücke (Energie-)Wende (einleiten), Kurs und Kurskorrektur, Umsteuern, das Ruder drehen, Fahrt aufnehmen, den Kurs fortführen, Rettungsanker sowie der Ausdruck rücksichtloser Industrialisierungskurs (B 90/GRÜNE 2002, S. 25) zu betrachten. Dem Bereich der nautischen Metaphorik bzw. den Schiffs-Metaphern sind auch die Ausdrücke verankern (B 90/GRÜNE 2002 (9), PDS 2003 (3), SPD 1989 (2), CDU 1971 (1), NPD 1973 (1), DKP 1969 (1)) und Verankerung (B 90/GRÜNE 2002 (2), DIE FRAUEN 2002 (1), CDU 1971 (1), FDP 1971 (1), DKP 1969 (1)) zuzuordnen; diese werden parteiübergreifend gebraucht.

\subsection{Wege-Metaphern}

In oben zitierter Äußerung 52 (d) lässt sich neben der Kampf-Metapher noch eine weitere Metapher nachweisen; der Ausdruck Meilensteine in der Verwendung diese Erfolge sind Meilensteine ist der Kategorie der Wege-Metaphern zuzuordnen, was durch die Verwendung des Ausdrucks opferreicher Weg auch explizit verdeutlicht wird. In den Parteiprogrammen lassen sich aber noch weitere Wege-Metaphern nachweisen, jedoch kann hier nur eine exemplarische Auswahl zitiert werden:

(55) (a) Die dargestellten Maßnahmen wie die Umverteilung der Einkommen, die Pro-fessionalisierung und existenzsichernde Bezahlung aller auf Menschen bezogenen Arbeiten bedeuten einen Schritt in Richtung einer solchen Umorientierung. (DIE FRAUEN 2002, S. 12)

(b) Ohne diesen Fortschritt hätte der Rückschritt freie Bahn. [...] Reformarbeit vollzieht sich oft in kleinen Schritten. Mehr noch als auf 
die Größe der Schritte achten wir auf die Erkennbarkeit der Richtung. [...] Unter Berücksichtigung der föderalen Zuständigkeiten und der unterschiedlich ausgestalteten Schulsysteme in den einzelnen Bundesländern müssen in den jeweiligen Ländern unterschiedliche Wege beschritten werden. [...] Die Verlängerung der gemeinsamen Grundschulzeit ist z.B. ein Schritt in die richtige Richtung. (SPD 1989, S. $7 / 52 / 96)$

(c) Deshalb wollen wir weitere Schritte gehen auf dem Weg hin $\mathrm{zu}$ einer flexiblen und sozial verträglichen Arbeitszeitpolitik, [...] (B 90/GRÜNE 2002, S. 70)

(d) Als Schritte auf dem Wege zu einer allgemeinen Abrüstung und zur Entspannung internationaler Beziehungen sind regionale Sicherheitssysteme im Rahmen der Vereinten Nationen aufzubauen. (Godesberger Programm 1959, S. 84)

(e) Die Lohnentwicklung hält nicht einmal Schritt mit dem Wachstum der Arbeitsproduktivität. (DKP 1969, S. 313)

(f) Die proletarische Revolution kann sich nur stufenweise, Schritt für Schritt, auf dem Golgathaweg eigener bitterer Erfahrungen, durch Niederlagen und Siege, zur vollen Klarheit und Reife durchringen. (KPD 1918/19, S. 117)

(g) In der Außen- und Sicherheitspolitik steht die Europäische Union an einem Scheideweg. [...] Wir sind der politischen Verfolgung, Ausgrenzung und Benachteiligung Ostdeutscher auf allen Ebenen entgegengetreten. (PDS 2003, S. 10/33)

(h) Die historischen Erfahrungen der Deutschen und ihre Entscheidung für ein gemeinsames Europa verbieten einen deutschen Sonderweg. (SPD 1989, S. 17)

Die Verwendung des hier dargestellten metaphorischen Grundmusters ist charakteristisch für die Sprachverwendung in den Parteiprogrammen und kann parteiübergreifend nachgewiesen werden; so ist von der Politik der kleinen Schritte (SPD 1989, S. 9) die Rede oder vom schrittweisen Aufbau/Übergang/Abbau. Weiterhin müssen neue Wege eröffnet werden (Godesberger Programm 1959, S. 82), neue Wege müssen gefunden werden (SPD 1989, S. 70/91), auf machtpolitische Sonderwege soll verzichtet werden (SPD 1989, S. 144/152) oder es wird neben dem Recht auf einen eigenen Entwicklungsweg (SPD 1989, S. 18) gefordert, den sozialistischen Weg zu beschreiten (SED 1963, S. 334). Weiterhin muss der Weg ein für allemal versperrt werden (SED 1963, S. 231) oder aber für ein bestimmtes Ziel in der Politik stehen alle Wege offen (SED 1963, S. 256).

Die Metaphern dieses Typs „fokussieren auf die Zielgerichtetheit politischen Handelns, blenden dabei jedoch zumeist neben alternativen Reise- 
und Handlungsmöglichkeiten auch die Landschaft aus, durch die gegangen wird. “ ${ }^{888}$

Folglich sind auch die Substantive Ebene und Sonderweg, das Verb entgegentreten sowie der Ausdruck an einem Scheideweg stehen der Textstellen (g) und (h) der Kategorie der Wege-Metaphern zuzuordnen.

\subsection{Pflanzen-Metaphern}

In den Parteiprogrammen können Pflanzen-Metaphern nachgewiesen werden. Dabei werden Wahrnehmungsmuster aus dem Bereich der Flora auf gesellschaftliche Bereiche übertragen. Insbesondere der Bereich der Wirtschaft wird als Pflanze dargestellt. Folgende Ausdrücke sind für dieses metaphorische Grundmuster charakteristisch: Blüte, aufblühen sowie Fäulnis. Auch die Verwendung von wachsen und schrumpfen in Äußerung (e) sowie reifen der Keime in Äußerung (b) folgen diesem Muster. Der Ausdruck Wachstum wird parteiübergreifend in den Programmen verwendet; so ist von einem gewachsenen Volkstum (NPD 1973, S. 359) und vom Wachstumsstreben der Wirtschaft (GRÜNE 1980, Kap. II, 1) die Rede und davon, dass nicht jedes Wachstum Fortschritt ist (SPD 1989, S. 41, die traditionelle Finanzierungsbasis schrumpft (B 90/GRÜNE 2002, S. 19), Schrumpfungsprozesse zu organisieren sind (B 90/GRÜNE 2002, S. 34) oder Wachstumsfelder ausgewählt werden (SPD 1989, S. 41). Ebenso gehören die Wörter Wurzel und wurzeln in Textstelle (f), die Wendung etwas an der Wurzel vernichten (KPD 1918/19, S. 108) sowie verwurzelt im Godesberger Programm (S. 69) dem Bereich der Pflanzen-Metaphorik an.

(56) (a) Die Entwicklung des staatsmonopolistischen Kapitalismus und die Schaffung internationaler Vereinigungen staatsmonopolistischer sind nicht in der Lage, die Labilität der kapitalistischen Wirtschaft zu überwinden und ihr eine längere Blüte zu sichern. [...] Gegenüber der aufblühenden sozialistischen Weltwirtschaft und dem erfolgreichen kommunistischen Aufbau in der Sowjetunion treten die den Imperialismus kennzeichnenden ausbeuterischen und reaktionären Züge, seine Fäulnis und sein Parasitismus, die Ausweglosigkeit seiner Lage und seine Menschenfeindlichkeit immer deutlicher zutage. [...] Die Fähigkeiten und Talente, die besten sittlichen Eigenschaften des freien Menschen werden zur Blüte gelangen und voll zur Entfaltung kommen. [...] Der Sozialismus wird die deutsche Nation durch friedliche Arbeit zur Blüte und Größe führen. (SED 1963, S. $217 / 218 / 333 / 337$ )

(b) In der das Bewußtsein revolutionierenden Tätigkeit der Brigaden der sozialistischen Arbeit und der sozialistischen Arbeitsgemeinschaften wachsen und reifen die Keime der kommunistischen Einstellung heran, daß die Arbeit zum Wohle der Gesellschaft erstes 
Lebensbedürfnis des Menschen und jede Arbeit, ob körperliche oder geistige, achtenswert und ehrenvoll ist. (SED 1963, S. 304/305)

(c) Die Entwicklung einer mündigen Gesellschaft, verantwortliche Politik und Wachstum der Wirtschaft sind ohne sie undenkbar. (CSU 1968, S. 235)

(d) Die fortdauernde Rezession bewirkt schrumpfende Märkte, sinkende wachstumsraten, Firmenzusammenbrüche und Massenarbeitslosigkeit. (DIE FRAUEN 2002, S.7)

(e) Wir wollen, daß wirtschaftliche Grundentscheidungen, vor allem darüber, was wachsen und was schrumpfen soll, demokratisch getroffen werden. (SPD 1989, S. 7)

(f) In unserer Geschichte wurzeln die Grundwerte des Demokratischen Sozialismus. [...] Unsere geschichtlichen Wurzeln. [...] Der Demokratische Sozialismus in Europa hat seine geistigen Wurzeln im Christentum und in der humanistischen Philosophie, in der Aufklärung, in Marxscher Geschichts- und Gesellschaftslehre und in den Erfahrungen der Arbeiterbewegung. (SPD 1989, S. 10)

\subsection{Bau- oder Gebäude-Metaphern}

Bau- oder Gebäude-Metaphern werden in allen Parteiprogrammen verwendet. Dabei scheint insbesondere die Verwendung des Ausdrucks Fundament typisch für die Sprachverwendung der Parteiprogramme zu sein (CDU 1971 (1), SED 1963 (7), CSU 1968 (1), Godesberger Programm 1959 (1)). Dennoch lassen sich Unterschiede in der Bedeutung bzw. der zeitlichen Bezugnahme nachweisen: Während die Verwendung der Metapher in Äußerung (a) zur Konzipierung politischer Sachverhalte und Zustände, die in der Zukunft liegen, dient, beschreibt die Metapher in Äußerung (b), dass eine Grundlage für eine weitere Entwicklung der Gesellschaft zum Zeitpunkt der Formulierung bereits gelegt ist. Somit ergibt sich aus der Verwendung in (a) eine Zielbestrebung der SED, während die SPD sich in (b) auf bereits Geleistetes beruft und dadurch für sich wirbt. Die Ausdrücke Grundlage in (c) und granitne Basis in (k) sind dem Bereich der Bau-Metaphern zuzuordnen (DKP 1969 (7), Gothaer Programm 1875 (1), FDP 1971 (9), CDU 1971 (19), SED 1963 (59), NSDAP 1920 (1), CSU 1968 (8), Godesberger Programm 1959 (5), Eisenacher Programm 1969 (1), PDS 2003 (25)); in Verbindung mit dem Attribut tragfähig ist der Ausdruck Teil der Brücken-Metaphorik als Unterkategorie der Bau-Metaphorik. Mit dem Ausdruck Errichtung des Gebäudes der Zukunft in Äußerung (l) ist die Gebäude-Metaphorik anschaulich verwirklicht. In die Kategorie der Bau-Metaphern fallen weiterhin die Ausdrücke ausbauen (d) und aufbauen sowie als Substantive Ausbau und Aufbau. Die Ausdrücke Grundpfeiler in Äußerung (e) und Eckpfeiler in Äußerung (f) und (h) gehören ebenfalls dem Bereich der Bau- oder Gebäude-Metaphorik 
an. Während die Ausdrücke der Äußerungen (a) bis (d) häufig vorzufinden sind lassen sich die beiden letztgenannten auch nur in diesen beiden Parteiprogrammen nachweisen. Ein zentraler Wert bei der Konzeptualisierung von Politik, der Währungen oder der Volkswirtschaft im Sinne der Bau- bzw. Gebäude-Metaphorik ist die Stabilität. Dieser Ausdruck findet sich parteiübergreifend in allen Parteiprogrammen, auch in den Formen stabil und Stabilisierung. Dabei ist anzumerken, dass grundsätzlich alles stabilisiert werden kann oder muss, von der Währung über die Preise und die Gesellschaft bis zum öffentlichen Haushalt, vom Energieverbrauch über eine stabile Futtergrundlage (SED 1963, S. 272) bis zum Sozialsystem. Auch die Wendung auf Säule( $n$ ) basieren in Äußerung (m) ist der Kategorie der Bau- oder Gebäude-Metaphorik zuzuordnen. Und letztlich sind Bausteine zum Errichten eines Gebäudes von grundlegender Bedeutung (vgl. Textstelle (n)); damit ist es möglich, Bastionen des Friedens zu schaffen (SED 1963, S. 231).

In den Bereich der Bau- oder Gebäude-Metaphorik gehört auch die Verwendungsweise des Verbs aushöhlen bzw. des Substantivs Aushöhlung in den zitierten Textstellen (g), (i), (j) und (k): Wenn Bündnisse oder Systeme ausgehöhlt werden, wird ihnen das Fundament oder die feste Grundlage entzogen, auf dem sie fußen. In diesem Sinne sind auch die Ausdrücke die Lebensgrundlage untergraben (PDS 2003, S. 4), das Grundgesetz untergraben (DKP 1969, S. 315) oder die Ausdrücke instabile Staaten (B 90/GRÜNE 2002, S. 163) sowie destabilisierende Wirkung (B 90/GRÜNE 2002, S. 170) zu verstehen. Die Äußerung (i) weist zwei weitere metaphorisch verwendete Ausdrücke auf: Durch die Darstellung, dass Freiheiten und Rechte abgebaut werden und das Grundgesetz untergraben wird orientiert sich die KPD bei der Formulierung an dem allgemeinen metaphorischen Muster "GOOD IS UP, BAD IS DOWN“389, das im Gegensatz zu den Ausdrücken der Bau- bzw. Gebäude-Metaphorik steht. Auch die Wendungen ruinöse Lohnkonkurrenz (DIE FRAUEN 2002, S. 17) und über den zusammensinkenden Mauern der kapitalistischen Gesellschaft (DKP $1918 / 19$, S. 109) sind dem Bereich der Gebäude-Metaphorik zuzuordnen.

(57) (a) Es ist das unverrückbare Ziel der Sozialistischen Einheitspartei Deutschlands, auf diesem festen Fundament - zusammen mit dem werktätigen Volk und für das deutsche Volk. - das neue, das sozialistische Zeitalter zu gestalten und seine lichten Höhen zu erstürmen. (SED 1963, S. 211)

(b) In einigen Ländern Europas wurden unter sozialdemokratischen Regierungen bereits die Fundamente einer neuen Gesellschaft gelegt. (Godesberger Programm 1959, S. 86) 
(c) Nur eine gegenseitige Toleranz, die im Andersglaubenden und Andersdenkenden den Mitmenschen gleicher Würde achtet, bietet eine tragfähige Grundlage für das menschlich und politisch fruchtbare Zusammenleben. (Godesberger Programm 1959, S. 81)

(d) Die Mitwirkung der Eltern in der Schulerziehung und eine Mitverwaltung der Schüler sollen an allen Schulen ausgebaut werden. (Godesberger Programm 1959, S. 82)

(e) Die brüderliche Freundschaft mit der Sowjetunion ist ein Grundpfeiler ihrer Außenpolitik. (SED 1963, S. 252).

(f) Die Verstetigung der kommunalen Einnahmen sind dabei ein ebenso entscheidender Eckpfeiler wie die Stärkung der Finanzautonomie durch die Verbesserung des Hebesatzrechtes. (B 90/GRÜNE 2002, S. 130)

(g) Durch die Bindung an den kommunistischen Block werden die Beziehungen zur nichtkommunistischen Welt schwer belastet, die Bündnisse ausgehöhlt und die Vereinigung der freien Völker und Staaten Europas gefährdet. (NPD 1973, S. 344)

(h) Diese Eckpfeiler des modernen Völkerrechts müssen ohne Ausnahme und gegenüber allen Staaten gewährleistet, verteidigt und durchgesetzt werden. (PDS 2003, S. 16)

(i) Das vom Grundgesetz vorgeschriebene parlamentarische System ist ausgehöhlt, der Bundestag zu einem Scheinparlament herabgesunken, [...]. Die demokratischen Rechte und Freiheiten werden abgebaut, das Grundgesetz untergraben. (DKP 1969, S. 315)

(j) Wesentliche Verschärfungen der Polizeigesetzgebung haben neben anderen Überwachungsmaßnahmen die Grundrechte ausgehöhlt. (GRÜNE 1980, Kap. V, 1.7)

(k) Wir haben uns gegen eine Aushöhlung der Asylpolitik gewandt. (PDS 2003, S. 33)

(1) $\quad[\ldots]$, die Erweiterung der deutschen Revolution zur Weltrevolution des Proletariats vermag allein die granitne Basis zu schaffen, auf der das Gebäude der Zukunft errichtet werden kann. (KPD 1918/19, S. 113)

(m) Unser derzeitiges Gesundheitssystem basiert maßgeblich auf zwei Säulen: einem patriarchal-ökonomischen und eienm patriarchal-medizinischen Ansatz. (DIE FRAUEN 2002, S. 38)

(n) Die Europäische Gemeinschaft ist ein Baustein einer regional gegliederten Weltgesellschaft. (SPD 1989, S. 16)

Man kann daher abschließend festhalten: Die in den Parteiprogrammen ermittelten und nachgewiesenen Metaphern „reflektieren Gedanken- 
gänge, mentale Muster, auf denen auch die praktische Politik aufbaut. “390 Jedoch sind die wenigsten der zitierten und diskutierten Metaphern originell. Die Metaphern sind auch nicht auf den Bereich der politischen Sprachverwendung beschränkt; vielmehr wird in diesem Bereich „,an die traditionellen Muster angeknüpft, die die Alltagssprache bereitstellt. “391

\subsubsection{Schlagwörter}

Neben dem Begriff Schlagwort sind in der linguistischen Analyse der politischen Sprachverwendung auch die Termini Leitwort oder Schlüsselwort gebräuchlich; Politologen wie BERGSDORF (1983) bevorzugen den letzteren und verstehen darunter "an einem Begriff festgemachte $\mathrm{Zu}$ kunftsentwürfe mit Vergangenheitsdeutungen, die politische Philosophien suggerieren, ohne sie deutlich zu explizieren." ${ }^{392}$ DIECKMANN (1969) spricht von Leitwort und Reizwort, wenn diese hinsichtlich ihrer Wertung unterschieden werden sollen; danach geht mit dem Leitwort eine positive und mit dem Reizwort eine negative Bewertung einher. In dieser Untersuchung wird jedoch der Terminus Schlagwort verwendet.

Die Verwendung von Schlagwörtern ist nicht auf den Bereich der politischen Sprachverwendung beschränkt, sondern kann in nahezu allen Bereichen der Sprachverwendung nachgewiesen werden; die Bedeutung eines allgemeinen Schlagwortes ist daher nicht scharf konturiert. In dieser Untersuchung ist jedoch von politischen Schlagwörtern als Erscheinungen der politischen Meinungssprache die Rede. Daher zeichnet sich deren Bedeutung durch einige Merkmale aus und wird insbesondere in der durch materielle und ideologische Interessengegensätze geprägten politischen Sprachverwendung deutlich, da diese Gegensätze zumeist im öffentlichen Meinungskampf zwischen den politischen Parteien ausgetragen werden:

Vor allem die Ausprägung Grundsatzprogramm [...] zeichnet sich durch gemeinsprachliche Lexik aus, die vor allem im Bereich der Schlagwörter normativ und emotiv geprägt ist und oft dem Vorwurf begrifflicher Unklarheit ausgesetzt ist. ${ }^{393}$

Die oben erwähnte normative und emotive Prägung einzelner Wörter geht zwar einher mit den Interessenkonflikten der gegnerischen Parteien, vor allem ist sie jedoch Ausdruck des mit sprachlichen Mitteln ausgefochtenen Kampfes um Wählerstimmen. Die Verwendung von Schlag-

390 Schäffner 1996, S. 153

391 Burkhardt 2003, S. 374

392 Bergsdorf, Wolfgang: Herrschaft und Sprache. Studie zur politischen Terminologie der Bundesrepublik Deutschland. Pfullingen 1983, S. 49

393 Klein 1996a, S. 202 
wörtern resultiert folglich aus den Handlungszielen, Macht und Einfluss zu erwerben oder zu erhalten: „Wo Interessenkonflikte bestehen und mit publizistischen Mitteln ausgetragen werden müssen, liegt der Griff zur plakativen Bezeichnung, zum einprägsamen, zugleich politische Bewertungen enthaltenden Schlagwort nahe. “394 Schlagwörter sind daher Ausdrücke, die eine "politisch aktuelle Tendenz" haben oder "irgendeine politische Gegebenheit schlaglichtartig charakterisieren." ${ }^{\text {"395 }}$

Auf formaler Ebene ist ein Schlagwort "ein kurzer Ausdruck unterhalb des Satzformats, im typischen Fall ein einziges Substantiv, allenfalls mit attributiver Erweiterung [...]." ${ }^{\prime 396}$ Ein Schlagwort verweist somit in komprimierter, griffiger Form auf einen komplexen politischen Sachverhalt; umfangreichere Nennungen wie der bereits analysierte Ausdruck öffentliches Interesse (Leerformel) können als „ideologische Formel“397 betrachtet werden, bei satzförmigen Gebilden (häufig als Ellipsen) ist von Parolen, Devisen oder Losungen auszugehen. Im Kapitel Wahlslogans/Wahlparolen in dieser Analyse werden die Parteiprogramme auf Schlagwörter als syntaktische Mehrwortwendungen hin untersucht.

Die Funktion des Schlagwortes liegt in der „Beeinflussung der öffentlichen Meinung im System der Meinungsbildung (Erziehung) und Meinungsänderung (Propaganda)“398, vorausgesetzt, das Sprechen vollzieht sich in der Öffentlichkeit und der Produzent der sprachlichen Äußerung hat die Absicht, die Öffentlichkeit zu beeinflussen; daher gehört das Schlagwort zur "Sprache der Überredung, aber nur da, wo sie öffentlich ist. “" ${ }^{399}$ Neben dieser emotiven Wirkung haben Schlagwörter die Funktion, politisch-ideologische Konzepte zu kristallisieren und dadurch zu simplifizieren. Aufgrund dieses Kondensierungsvorgangs und des einhergehenden Eigenwerts ist ein Schlagwort „durch angelagerte Begleitgefühle charakterisiert" 400 und es bedarf kaum mehr des Kontextes und bleibt auch in isolierter Verwendungsweise verstehbar.

Hinsichtlich der Verwendungsweise von Schlagwörtern ist festzuhalten, dass ein Schlagwort an sich nicht existiert, sondern erst durch eine bestimmte Verwendungsweise zu einem solchen wird. In Anlehnung an FERDINAND DE SAUSSURES (1967) Differenzierung zwischen langue (Spra-

\footnotetext{
394 Burkhardt 1996, S. 91

395 Bachem 1979, S. 63

396 Ickler, Theodor: „Zur Semantik des politischen Schlagwortes (und anderer Wörter).“ In: Sprache und Literatur in Wissenschaft und Unterricht (Band 65). München/Paderborn 1990, S. 11

397 Ickler 1990, S. 11

398 Dieckmann 1969, S. 102

399 Dieckmann 1969, S. 102

400 Dieckmann 1969, S. 104
} 
che/sprachliches System einer Sprachgemeinschaft) und parole (Sprechen/Realisierung menschlicher Sprache $)^{401}$ ist das Schlagwort eine Erscheinung der parole, nicht der langue. Theoretisch kann daher jedes Wort innerhalb eines bestimmten Diskurses als Schlagwort gebraucht werden. Da viele Schlagwörter aus Fachsprachen oder Wissenschaftssprachen stammen, existiert neben einem bestimmten Schlagwort in der Regel "eine nicht-,schlagende' Dublette, oft sogar als Fachwort [...]." ${ }^{402}$ Das "Schlagende" oder "Nicht-Schlagende" eines Schlagwortes besteht in dessen agitatorischer Funktion: Das Schlagwort wird verwendet, um „,im Hörer oder Leser eine bestimmte Handlungsdisposition zu erzeugen. “403 Daher hat ein Schlagwort neben der deskriptiven Bedeutung auch eine affektive und appellative sowie eine emotionale Bedeutung oder Wirkung mit dem Ziel, den Rezipienten zu mobilisieren; „das Moment des Schlagens und Treffens, das in der Wortbildung steckt, kann durchaus wörtlich genommen werden." ${ }^{404}$

So lassen sich für ein Schlagwort folgende Eigenschaften und Funktionen zusammenfassen:

Unbestimmtheit und Verallgemeinerung, scheinbare Klarheit und sekundäre Nähe, Gefühlsbelastung usw. In den Schlagwörtern werden die Programme kondensiert; sie erheben Relatives zu Absolutem, reduzieren das Komplizierte auf das Typische, Überschaubare, Einfach-Gegensätzliche und bilden dadurch bipolare Wortschatzstrukturen aus; sie bringen das Abstrakt-Ferne sprachlich nahe und geben der Meinungssprache ihre emotionellen Obertöne. ${ }^{405}$

\subsection{Fahnen- und Stigmawörter}

Die oben erwähnte bipolare Wortschatzstruktur resultiert aus der Verwendung der Schlagwörter. So können Schlagwörter (i) als Fahnenwörter oder (ii) als Stigmawörter fungieren, je nachdem, ob das eigene politische Programm des Sprechers bezeichnet wird (Fahnenwort) oder ob das Schlagwort als Schimpfwort oder Negativ-Bezeichnung für den politischen Gegner (Parteien, Mitglieder, Ziele, Werte) verwendet wird (Stigmawort). Durch die Verwendung eines Schlagwortes in der jeweiligen Verwendungsweise wird sowohl die Bejahung durch POSITIVIEREN einerseits als auch die Ablehnung einer bestimmten politischen Pro-

401 Saussure, Ferdinand de: Grundfragen der allgemeinen Sprachwissenschaft. Berlin/New York ${ }^{32} 2001$, S. 11ff.

402 Ickler 1990, S. 11

403 Ickler 1990, S. 12

404 Dieckmann 1969, S. 104

405 Dieckmann 1969, S. 103 
grammatik andererseits durch NEGATIVIEREN zum Ausdruck gebracht;

Stigmawörter, die gleichzeitig als Fahnenwörter der anderen Seite gebraucht werden (Fahnenwörter/Stigmawörter), haben zumindest überschneidende Extension (im Grenzfall Extensionsgleichheit). Diese Extensionsüberschneidung gewährleistet, daß im referentiellen Gebrauch ein und dieselbe ,Sache' von der einen Seite ,auf die Fahne geschrieben' und von der anderen Seite ,stigmatisiert' werden kann. ${ }^{406}$

Der Sachverhalt Eingliederung eines Staates in einen anderen Staat oder in ein Staatenbündnis kann sowohl durch das Fahnenwort Beitritt (in Anlehnung an den Grundgesetztext Art. 23) bezeichnet werden als auch durch das Stigmawort Anschluss (in Analogie zum "Anschluß" Österreichs an das Deutsche Reich im Jahre 1938). Während der Ausdruck Beitritt zur Beschreibung eines aktiven Prozesses dient, wird mit der Bezeichnung Anschluss die Passivität des jeweiligen Staates ausgedrückt. Der Ausdruck Anschluss wird dabei „zur Vorbehaltsvokabel für Kritiker, die den Vereinigungsprozeß als übereilte Prozedur betrachteten." ${ }^{407}$ Der beitretende Staat wird vom politischen Gegner zum angeschlossenen Staat degradiert und verliert somit seine Rolle als Agens.

(58) (a) Im Bewußtsein der Folgen gingen sie den Weg der einseitigen Bindung an die Westmächte, der Remilitarisierung und des $\underline{\text { An- }}$ schlusses an die NATO. (DKP 1969, S. 311)

(b) Die Europäischen Gemeinschaften sollen durch die Aufnahme beitrittswilliger Staaten erweitert werden, ohne daß die Ziele, die Ergebnisse des Integrationsprozesses und die Funktionsweise der Gemeinschaften beeinträchtigt werden. (CDU 1971, S. 50)

(c) Die ostdeutschen Länder haben eine Brückenfunktion zu den Beitrittsländern der EU in Osteuropa. (B 90/GRÜNE 2002, S. 49)

(d) Wir lehnen es aber ab, die Frage der Kompetenzabgrenzung zu missbrauchen, um sich angesichts des Beitritts ärmerer Länder und Regionen aus der europäischen Solidarität $\mathrm{zu}$ stehlen. (B 90/GRÜNE 2002, S. 154)

(e) Die Erweiterung der Europäischen Union, die mit den jetzt vorgesehenen Beitritten fast zu einer Verdoppelung der Mitglieder führen wird, [...]. (B 90/GRÜNE 2002, S. 156)

(f) Auch wenn die Beitrittsverhandlungen mit diesen Staaten erfolgreich abgeschlossen sind, [...]. (B 90/GRÜNE 2002, S. 157)

406 Strauß 1986b, S. 109

407 Stötzel, Georg/Wengeler, Martin: Kontroverse Begriffe. Geschichte des öffentlichen Sprachgebrauchs in der Bundesrepublik Deutschland. Berlin/New York 1995, S. 331 
Auch die Wörter Sozialismus, Kommunismus, Klassenkampf oder Kapitalismus können - abhängig vom Produzenten - sowohl als Fahnen- als auch als Stigmawort gebraucht werden; daher hängt die eindeutige Lesart vom Kontext der Verwendungsweise des Ausdrucks ab. Diese Tatsache widerspricht der von DIECKMANN (1969) formulierten These der Kontextunabhängigkeit eines Schlagwortes. Schlagwörter - sowohl Fahnen- als auch Stigmawörter - sind vielmehr "stets auf die emotionale und intellektuelle Beeinflussung der Öffentlichkeit gerichtet“ und insofern „immer auch motiviert von dem Willen, die intellektuelle Wirklichkeitserfahrung zu lenken." ${ }^{408}$ Es handelt sich beim Gebrauch von Ausdrücken als Schlagwörter folglich um „ideologisch gefärbte öffentlich-exponierte Abstrakta in appellativ-emphatischer Verwendung. ${ }^{409}$

Fahnen- oder Stigmawörter gehören somit wie die bereits behandelten Miranda und Anti-Miranda dem Bereich der ideologiegebundenen Lexik an. Das ideologische Lexikon ließe sich danach mit folgendem Schaubild veranschaulichen:

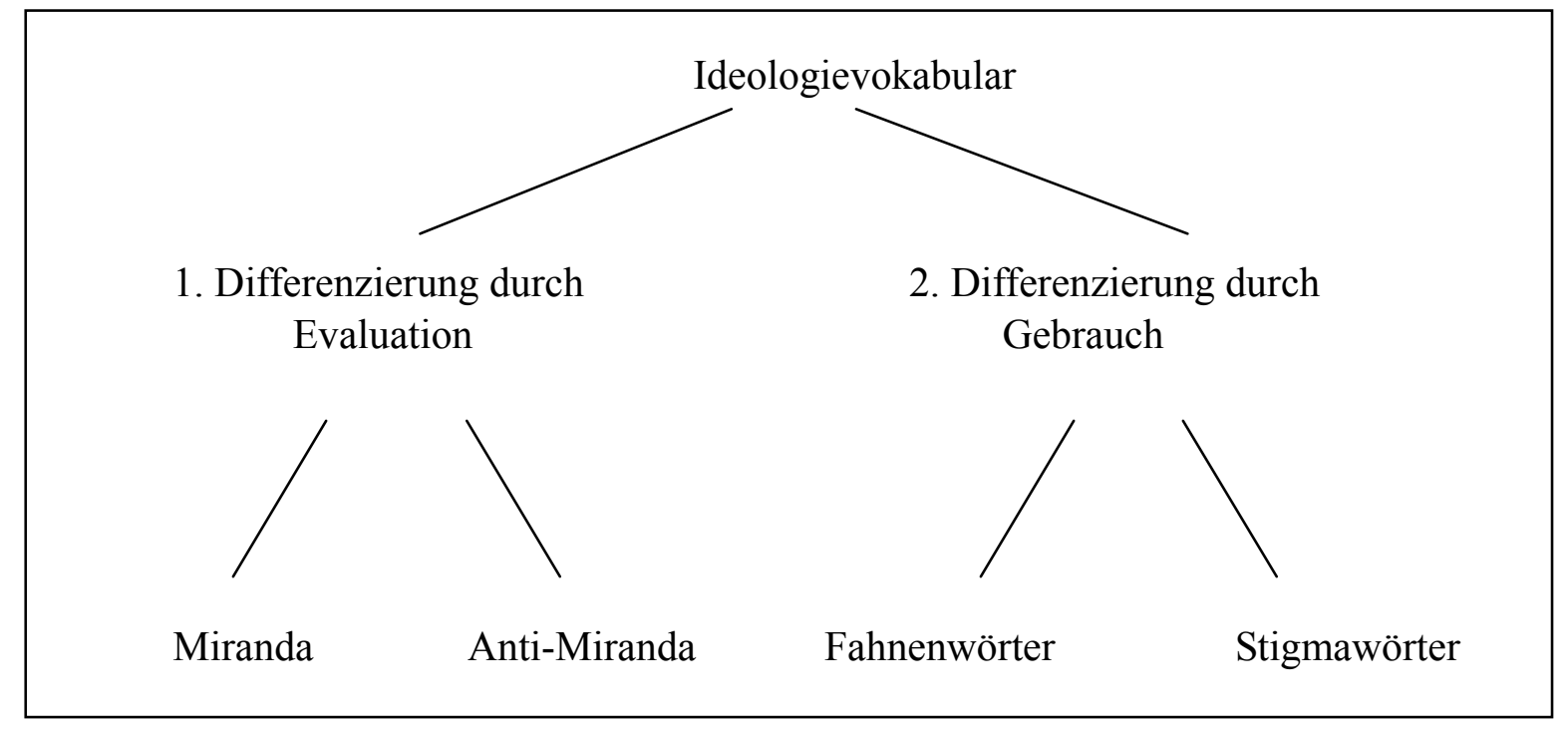

Abbildung 7: (vgl. GIRNTH 2002, S. 55)

Auch Wörter wie Demokrat, Pazifist, Sozialist oder Kommunist haben

zwar rein sachlich-deskriptive denotative Komponenten, aber weil der Verwender dieser Wörter selbst immer dem einen oder anderen Lager angehört, ist ein wertneutraler Gebrauch, der keine Assoziationen und impliziten Wertungen weckt, so gut wie ausgeschlossen. ${ }^{410}$

\footnotetext{
408 Strauß/Haß/Harras 1989, S. 33

409 Burkhardt 2003, S. 354

410 Burkhardt 1988, S. 341
} 
Exemplarisch können die Kontextabhängigkeit und die impliziten Wertungen eines Schlagwortes am Ausdruck Kommunisten zur Bezeichnung der Anhänger dieser Ideologie verdeutlicht werden; dabei verwendet die SPD den Ausdruck Kommunisten als Stigmawort in Realisierung des kommunikativen Verfahrens DELEGITIMIEREN, in der Gebrauchsweise der DKP und der SED erscheint Kommunisten als Fahnenwort in Realisierung der kommunikativen Verfahren SOLIDARISIEREN und INTEGRIEREN sowie WERBEN:

\begin{tabular}{|l|l|}
\hline Kommunisten als Stigmawort & \multicolumn{1}{|c|}{ Kommunisten als Fahnenwort } \\
\hline Die Kommunisten unterdrücken & Er [der Imperialismus und Antikommunismus] \\
die Freiheit radikal. (Godesberger & richtet sich gegen die Kommunisten und die Arbei- \\
Programm 1959, S. 86) & terklasse, gegen alle demokratischen und verständi- \\
Die Kommunisten missbrauchten & gungsbereiten Kräfte, gegen die ganze friedliebende \\
dabei die in der Zeit der national- & Bevölkerung Westdeutschlands und selbst gegen \\
sozialistischen Diktatur entstan- & jene Kräfte in der Großbourgeoisie, die normale \\
dene Sehnsucht, die Spaltung der & friedliche, ökonomische, politische und kulturelle \\
Arbeiterbewegung möge im & Beziehungen mit den sozialistischen Ländern an- \\
Zeichen einer demokratischen & streben. (SED 1963, S. 245) \\
Erneuerung überwunden wer- & Wir Kommunisten sehen im Kampf für die Gleichbe- \\
den. $(S P D$ 1989, S. 9) & rechtigung der Frau, gegen ihre Benachteiligung in \\
der Gesellschaft eine der besten Traditionen der \\
Arbeiterbewegung. (DKP 1969, S. 337)
\end{tabular}

Deutlich wird bei der Gegenüberstellung der Textstellen auch, dass die Verwendung von Fahnen- und Stigmawörtern zur gegenseitigen Abgrenzung dient: Als abstrakte, politisch-programmatische Kurzformel hat das Schlagwort "strategischen Charakter insofern, als es für längere Zeit geeignet ist, den eigenen Standpunkt in gedrängter Form darzustellen und sich vom Gegner abzugrenzen." ${ }^{411}$

Eine stigmatisierende Bezeichnung des politischen Gegners wird auch durch die Verwendung des Adjektivs reaktionär ermöglicht; in den untersuchten Parteiprogrammen findet sich das Adjektiv mit folgender Häufigkeit: NPD 1973 (1), SPD 1989 (1), SED 1963 (17), DKP 1969 (12) und Gothaer Programm (1). Zur weiteren Betonung und Stigmatisierung kombiniert die SED das Adjektiv reaktionär häufig mit den Adjektiven imperialistisch, volksfeindlich, aggressiv und chauvinistisch oder verwendet reaktionär im Superlativ (c). Insbesondere bei Betrachtung der Verwendungsweisen von NPD (a) und im Gothaer Programm (b) fällt auf, dass der Ausdruck reaktionär gegensätzliche Positionen beschreibt und als Stigmawort zweier unterschiedlicher Betrachtungsweisen fungiert. Die Distanzierung wird bei der NPD weiterhin durch den Gebrauch des

411 Klaus 1971, S. 134 
Attributs gemeinschaftszerstörend und durch den Ausdruck extremen Linken verdeutlicht:

(59) (a) Sie sprechen von ,Reformen' und betreiben unter dem ständig wachsenden Druck revolutionierender Kräfte der extremen Linken den Rückschritt der gesellschaftlichen Entwicklung durch Wiederbelebung eines gemeinschaftszerstörenden, reaktionären Klassenkampfes. (NPD 1973, S. 343)

(b) Die Befreiung der Arbeit muß das Werk der Arbeiterklasse sein, der gegenüber alle anderen Klassen nur eine reaktionäre Masse sind. (Gothaer Programm 1875, S. )

(c) Der westdeutsche Staat ist die militaristisch-klerikale Diktatur der besonders mit dem amerikanischen und französischen Monopolkapital verflochtenen reaktionärsten Monopolgruppen. In Westdeutschland üben die reaktionärsten und aggressivsten imperialistischen und militaristischen Ultras die Macht aus. (SED 1963, S. 244)

Die Verwendung von Anführungszeichen (vgl. Reformen in Äußerung (a) oben) ist ein Mittel um dem politischen Gegner das Recht zur Verwendung eines Wortes abzusprechen. Die Einfügung solcher (hier: distanzierender und ironisierender) Anführungszeichen verwandelt ein Fahnenwort in ein Stigmawort und verwirklicht daher wie das Stigmawort das kommunikative Verfahren DELEGITIMIEREN:

So gehorcht es also zwingender politischer Logik, daß man sich, auf weltpolitischer Bühne, wechselseitig die Legitimität des Anspruchs auf den Gebrauch zentraler politischer Vokabeln streitig macht. Vorzugsweise verfährt man dabei so, daß man in Zitationen einschlägiger Verlautbarungen des Gegners die von ihm darin konkurrierend in Anspruch genommenen großen Worte wie Freiheit oder Demokratie etc. in Anführungsstriche setzt. Die Anführungsstriche sind das gewöhnliche Mittel sprachpolitischer Illegitimitätserklärung von Wortgebräuchen. ${ }^{412}$

Nachfolgend sind einige der durch Anführungszeichen gekennzeichnete Stigmawörter in ihren Verwendungsweisen der Parteiprogramme wiedergegeben. Dabei wird insbesondere bei Betrachtung der Äußerung (c) ersichtlich, wie sich der Produzent der sprachlichen Handlung (hier die Partei DIE FRAUEN als Verfasserkollektiv) durch die Verwendung von Anführungszeichen von einem bestimmten Ausdruck distanziert; die FRAUEN als feministische Partei scheinen dadurch deutlich zu machen, dass es Männerlehrberufe nicht geben kann und sprechen diesem Ausdruck weiterhin dessen Daseinsberechtigung ab.

412 Lübbe, Hermann: „Der Streit um Worte. Sprache und Politik.“ In: Holzfeuer im hölzernen Ofen. Aufsätze zur politischen Sprachkritik. Hg. v. Hans Jürgen Heringer. Tübingen 1982, S. 66 
(60) (a) Der imperialistischen ,Europa-Idee' stellt die Sozialistische Einheitspartei Deutschlands die gleichberechtigte Zusammenarbeit aller Völker und Staaten auf der Grundlage der Achtung und Souveränität und der friedlichen wirtschaftlichen Beziehungen gegenüber. (SED 1963, S. 253)

(b) Zwei Jahrzehnte lang haben bürgerliche Regierungen die deutsche Einheit der westlichen ,Integration' geopfert; nun sind die Linken dabei, ganz Deutschland dem Sowjetimperialismus auszuliefern. (NPD 1973, S. 342)

(c) Hierdurch werden Mädchen in ,Männerlehrberufen' benachteiligt und müssen oft auch noch sexistisches Verhalten von Vorgesetzten und Gesellen erdulden. (DIE FRAUEN 2002, S. 44)

In diesem Zusammenhang ist das Adjektiv sogenannt in attributiver Verwendung zu erwähnen. Die Verwendung von sogenannt im Gegnerbezug ist ein häufiges Mittel und dient demselben Ziel wie die Einfügung von Anführungszeichen (Distanzierung und Stigmatisierung des politischen Gegners). Im Eigenbezug können Anführungszeichen und das Adjektiv sogenannt aber auch zur bloßen Kennzeichnung eines spezifischen Terminus dienen. LEINFELLNER (1971) sieht durch Hinzufügen von sogenannt unter gegebenen pragmatischen Umständen eine euphemistische Wendung verwirklicht: Der Ausdruck sogenannte DDR „verdeckte in der politischen Sprache der Bundesrepublik für lange Zeit die Tatsache, daß der DDR der Charakter eines Staates zukomme."413 Der Ausdruck Deutsche Demokratische Republik als Name oder Bezeichnung für ein Land oder einen Staat wird somit zu einer „uneigentlichen Redeweise“414 reduziert. Aus diesem Grund kann der Gebrauch von sogenannt in der politischen Sprachverwendung auch dem Erzeugen von Sichtweisen dienen (vgl. Kapitel 2.2.1.10). Nachfolgend sollen jedoch Beispiele in der stigmatisierenden Verwendungsweise wiedergegeben werden.

(d) Eine durch sogenannte ,Liberalisierung' bewirkte Aufweichung zerstört diese Rechts- und Wertordnung und führt zum Untergang des demokratischen Rechtsstaates. (NPD 1973, S. 350)

(e) Mit Hilfe von staatsmonopolistischen Maßnahmen - der sogenannten Entwicklungshilfe und anderem - sind die Imperialisten bestrebt, die Rohstoffressourcen der bisherigen kolonialen und abhängigen Länder auszunutzen, um hohe Profite zu erzielen und diese Länder in Abhängigkeit zu halten. (SED 1963, S. 220)

413 Leinfellner, Elisabeth: Der Euphemismus in der politischen Sprache. Berlin 1971, S. 92/93

414 Leinfellner 1971, S. 93 
Schlagwörter lassen sich auch als „,aggregierte Symbole“ 415 fassen, jedoch ist hierbei zu fragen, welche Faktoren das Symbol hervorrufen. Solche Faktoren sind die Zuschreibungen, die an den begrifflichen Inhalt (das Denotat) angefügt werden. Bestimmte Schlagwörter werden dann zu Wertwörtern, "die entweder als Schibboleths in unterschiedliche ideologische Systeme gehören oder aber so hohe Werte ausdrücken, daß sie von verschiedenen Gruppen adaptiert, aber ideologisch unterschiedlich ausgedeutet und damit ,ideologisch polysem' werden." ${ }^{416}$ In der Sprachwissenschaft werden unter Schibboleths "sprachliche Erkennungszeichen verstanden, die Rückschlüsse auf die dialektale, soziolektale, gruppen-, fach- oder sondersprachliche Herkunft des Sprechers erlauben. “417 Danach erfüllen gruppenspezifische Wortgebräuche und Bedeutungen eine gruppenintegrative Funktion und dienen dazu, Zusammengehörigkeitsgefühle zu erzeugen. Dieser Definition folgend sind die Ausdrücke Kommunismus oder die Kommunisten aggregierte Symbole bzw. Wertwörter im Sinne ideologischer Polysemie, da diese Ausdrücke politische Konzepte und Sehweisen beinhalten und von verschiedenen Gruppierungen/Parteien auf unterschiedliche Weise bewertet werden, obwohl der begriffliche Inhalt (das Denotat) gleich oder annähernd gleich ist:

\begin{abstract}
Man denke etwa an das Symbol ,Kommunismus', das einmal im Bereich der sozialistischen Staaten und der kommunistischen Arbeiterparteien der kapitalistischen Länder sowie breiter Volksmassen in den jungen Nationalstaaten einen positiven Sinn hat und das Bild einer leuchtenden Zukunft in sich trägt, während in den kapitalistischen Ländern die herrschenden Mächte alles versuchen, um mit den Mitteln der Presse, des Rundfunks usw. dem Symbol ,Kommunismus' den Charakter einer Drohung zu verleihen. ${ }^{418}$
\end{abstract}

In diesen Bereich fällt auch der Ausdruck Revolution, der in den Parteiprogrammen auf diese Weise verwendet wird. Die Ausdrücke Freiheit, Demokratie oder Selbstbestimmung unterscheiden sich jedoch von den zuvor genannten Ausdrücken, da hier der begriffliche Inhalt unterschiedlich ist, die Parteien der unterschiedlichen Ideologien als Produzenten der sprachlichen Äußerungen die Sachvorstellung der Begriffe aber gleich bewerten. Die dritte Verwendungsweise von politischen Ausdrücken als aggregierte Symbole geht mit unterschiedlichen begrifflichen Inhalten (Denotaten) und gleichzeitiger unterschiedlicher Bewertung einher, was bei der Verwendung der Ausdrücke Diktatur oder parteilich zum Ausdruck kommt.

\footnotetext{
415 Klaus 1971, S. 57

416 Burkhardt 2003, S. 354

417 Burkhardt 1988, S. 339

418 Klaus 1971, S. 58
} 
Das Kennzeichen der ideologischen Polysemie ist in einem beliebigen Ausdruck dann verkörpert, wenn dieser als Fahnenwort einer Gruppe und zugleich als Stigmawort einer anderen Gruppe verwendet werden kann, was insbesondere auf die folgenden Ausdrücke der Parteiprogramme zur Bezeichnung für Ideologien oder Regierungssysteme zutrifft: Nationalismus, Föderalismus, Kommunismus, Kapitalismus, Diktatur, Republik, Internationalismus, Sozialismus, Patriotismus, Zentralismus, Revisionismus, Bürokratismus oder Kollektivität. Zwar haben all diese Ausdrücke einen neutralen begrifflichen Inhalt und einen deskriptiven Gebrauch in den Bereichen der Philosophie, der Soziologie oder in der Politikwissenschaft, in der politischen Sprachverwendung der Parteiprogramme gehen jedoch ideologisch bedingte Deutungen und Wertungen einher. Die unterschiedlichen ideologischen Auslegungen desselben Bedeutungskerns haben in den meisten Fällen zur Folge, „daß zusätzliche Nebenvorstellungen als weitere, aber jeweils gruppenspezifische semantische Merkmale hinzutreten. Diese Annahme wird besonders deutlich bei Betrachtung der Verwendungsweisen des Ausdrucks Sozialismus, je nachdem, ob die SPD, die SED oder die CDU den Ausdruck verwendet. Es handelt sich bei den Verwendungsweisen von Sozialismus um „perspektivisch verschiedene Ausdeutungen desselben Begriffs “419, d.h. derselbe Ausdruck wird von politischen Gegnern in unterschiedlicher Bedeutung verwendet. Dagegen besteht bei den zuvor besprochenen Ausdrücken Anschluss und Beitritt derselbe Sachverhalt, die Bezeichnung wird jedoch semantisch unterschiedlich akzentuiert, d.h. aus unterschiedlichen Perspektiven bezeichnet und bewertet und unterscheidet sich somit aufgrund der semantischen Relationen von den anderen, zuvor genannten ideologischen Polysemen. KLEIN (1991b) unterscheidet daher zwischen der „Begriffsprägung“ (im Sinne einer alle Ausdrucksund Inhaltsaspekte betreffenden Neuprägung), der "Bezeichnungskonkurrenz", der "deskriptiven Bedeutungskonkurrenz", der "deontischen Bedeutungskonkurrenz" und der „Konkurrenz um konnotativen Glanz ${ }^{\prime 420}$, um die beiden oben genannten Phänomene terminologisch erfassen zu können.

An dieser Stelle beschränkt sich die Untersuchung auf die Bezeichnungsund Bedeutungskonkurrenz: „Bezeichnungskonkurrenz liegt vor, wenn umstritten ist, welche von mindestens zwei Bezeichnungen für einen Sachverhalt die ,richtige' ist", währenddessen bei der Bedeutungskon-

419 Burkhardt 2003, S. 357

420 Klein, Josef: „Kann man ,Begriffe besetzen'? Zur linguistischen Differenzierung einer plakativen politischen Metapher." In: Begriffe besetzen. Strategien des Sprachgebrauchs in der Politik. Hg. v. Frank Liedtke/Martin Wengeler/Karin Böke. Opladen 1991b, S. 50ff. 
kurrenz „die Bedeutung ein und desselben Ausdrucks umstritten“421 ist. Eine Bezeichnungskonkurrenz liegt beispielsweise bei der positiven Bezeichnung Soziale Marktwirtschaft (CSU 1968, FDP 1971, CDU 1971, SPD 1989, B 90/GRÜNE 2002) einerseits und der negativen Bezeichnung Kapitalismus (KPD 1918/19, SED 1963, DKP 1969, FDP 1971, SPD 1989, B 90/GRÜNE 2002, PDS 2003) andererseits vor, die sich beide auf denselben Sachverhalt Wirtschaftsform der Bundesrepublik Deutschland beziehen, und die Bedeutung der Sozialpartnerschaft, die in dem positiven Ausdruck sozialpartnerschaftliche Traditionen (B 90/GRÜNE 2002, S. 47) steckt, bezieht sich ebenso auf das Verhältnis von abhängig Beschäftigten und Kapitaleignern wie der negative Ausdruck Klassenkampf (KPD 1918/19, SED 1963, DKP 1969, NPD 1973). Bezeichnungskonkurrenz kann jedoch auch innerparteilich nachgewiesen werden; so bezeichnen die GRÜNEN den Sachverhalt Dienst in der Bundeswehr in ihrem Programm von 1980 als Kriegsdienst und in ihrem Programm von 2002 als Friedensdienst und Wehrdienst. Eine Bedeutungskonkurrenz ergibt sich insbesondere aus der Verwendung von Hochwertwörtern oder Grundwerten wie Demokratie, Freiheit, Solidarität oder Gerechtigkeit, da die Bedeutungen solcher Ausdrucke parteiübergreifend stabil sind und die jeweiligen Parteien dennoch versuchen, die „Begriffe zu besetzen“.

Die globale Kategorie der Schlagwörter lässt sich neben der Differenzierung in Fahnen- und Stigmawörter noch in weitere typologische Unterscheidungen untergliedern. Nachfolgend sind die von BURKHARDT (2003) formulierten terminologischen Differenzierungen als Unterkategorien ${ }^{422}$ Zeitgeistwörter, Hochwertwörter, Unwertwörter, Programmwörter, Stich- oder Themawörter und Scheltwörter wiedergegeben und mit Beispielen aus den untersuchten Parteiprogrammen unterfüttert.

\subsection{Zeitgeistwörter}

Schlagwörter sind mit ihren jeweiligen charakteristischen Diskursthemen an einen bestimmten Zeitabschnitt gebunden. Diese Zeitgeistwörter sind Schlagwörter, die auch außerpolitisch und überparteilich erscheinen können, wie beispielsweise: Parteienskepsis, Politikverdrossenheit, Umweltaußenpolitik, Globalisierung, Informationstechnologie, Medienkompetenz, Selbstverwirklichung sowie der Ausdruck Entfaltung der Persönlichkeit. Auch der Ausdruck Arbeitslosigkeit gehört in diese Kategorie, obwohl dieser nicht außerpolitisch ist.

\footnotetext{
421 Klein 1989, S. 17

422 Burkhardt 2003, S. 357/358
} 


\subsection{Hochwertwörter}

Die Unterkategorie der Hochwertwörter beinhaltet überparteiliche Schlagwörter, die nicht zeitgebunden über einer beliebigen zeitgenössischen politischen Diskussion stehen. Charakteristisch für diese Wörter sind die abstrakte Unverbindlichkeit und die Tatsache, dass die meisten dieser Ausdrücke parteiübergreifend verwendet werden. Dennoch sind einige der Hochwertwörter charakteristisch für die Sprachverwendung der jeweiligen Partei, beispielsweise Wohlstand, Abschreckung, Aufschwung oder Sicherheit auf der konservativen Seite (CDU/CSU), Soziale Gerechtigkeit oder Solidarität auf der sozialdemokratisch-sozialistischen Seite (SPD/PDS) und Umweltbelastung, Umweltschutz oder Zukunft auf der ökologisch-alternativen Seite (GRÜNE). Insbesondere hinsichtlich des Hochwertworts Solidarität besteht eine deskriptive Bedeutungskonkurrenz zwischen der Verwendung in der Tradition der Arbeiterbewegung und in konservativ-christlicher Deutung. Daher sind Hochwertwörter zumeist die Schlüssel- oder Kernwörter der jeweiligen Doktrin.

Auch die bereits besprochenen Grundwerte sind dieser Kategorie zuzuordnen. Folgende Ausdrücke lassen sich in den analysierten Parteiprogrammen nachweisen und werden parteiübergreifend verwendet: Deutschland, Mitte [,Diese Erfahrungen reichen bis weit in die Mitte der Gesellschaft." (B 90/GRÜNE 2002, S. 63)], Gemeinschaft, Individualisierung, Gesundheit, modern, Freiheit, Toleranz, Fairness, Wirtschaftsaufschwung, Kultur, Bildung, Volk, Würde und Menschenwürde sowie Frieden.

\subsection{Unwertwörter}

Die Unterkategorie der Unwertwörter umfasst parteiübergreifend negativierende Wörter, die als Schlagwörter verwendet werden, wie beispielsweise Terrorist, Antisemitismus, Rechtsextremismus, Rassismus, autoritär, Barbarei, Chaos, totalitär sowie Regime.

\subsection{Programmwörter}

Programmwörter sind parteiliche Begriffe, die zeitgebunden sind und die aktuelle oder mittelfristige Konzepte der einzelnen Parteien bezeichnen. Diese Schlagwörter bezeichnen auch politische Lager und dienen der Verständigungsökonomie. Zu ihnen zählen: Entspannung, Abrüstung, Qualitäts- und Qualifikationsoffensive, Bildungs-, Finanz- und Verwaltungsreform sowie Reform des Gesundheitssystems, Solidaritätsabgabe. 


\subsection{Stich- oder Themawörter}

Eine weitere Unterkategorie stellen Stichwörter oder Themawörter dar. Es handelt sich dabei um Wörter, die lexikalisch lediglich besonders wichtige Aspekte des Bezeichneten hervorheben und die dabei voraussetzen, dass der Rezipient aufgrund seiner Informiertheit den Ausdruck entsprechend wahrnimmt; folglich haben die Schlagwörter dieser Kategorie häufig eine zielgruppenspezifische Orientierung. Es handelt sich bei diesen Schlagwörtern um "metonymisch fokussierende und insofern ebenfalls sprachökonomisch bedingte Verkürzungen. ${ }^{423}$ In diese Kategorie fallen die Ausdrücke Altschuldenregelung, Radikalenerlaß, vor die Auswahl zwischen ,Harrisburg' und "Hiroshima' gestellt sein [stellvertretend für Atomstaat oder Atomkrieg und alle Assoziationen, die bei der Rezeption des Ausdrucks Hiroshima entstehen], Globalisierung, \& 218 [Abtreibung, Schutz des ungeborenen Lebens], 11. September (2001) [Terroranschlag auf das World Trade Center in Amerika und alle Assoziationen, z.B. 3000 Tote, Terrorismus, verwundete Nation, Flugzeugentführung, Flugangst usw.], Tschernobyl [nuklearer Supergau und alle entstehenden Assoziationen, z.B. atomarer Niederschlag, verunreinigte Lebensmittel, dauerhafte Verseuchung ganzer Landstriche, verendetes Vieh, kranke Menschen, marode Industrieanlagen usw.], Standort Deutschland [in den Parteiprogrammen auch als Industriestandort, Innovationsstandort, Wirtschaftsstandort usw.], Kosovo, Dritte Welt.

\subsection{Scheltwörter}

Schlagwörter können auch als die gegnerische Politik abwertende Scheltwörter verwendet werden; zumeist enthalten diese Schlagwörter den Vorwurf moralischer Verfehlung, wie beispielsweise Lüge(n), Staat des Unrechts, antinationaler Staat oder der Ausdruck betrogenes Volk [dabei ist die gegenwärtige Regierung als Betrüger impliziert].

An einigen der angegebenen Ausdrücke kann beispielhaft aufgezeigt werden, wie sich aufgrund von hinzugefügten Attributen ein Schlagwort zu einer begriffsarmen Leerformel verändern kann; so wird beispielsweise der bereits zitierte Ausdruck Zentralismus zur Bezeichnung einer Regierungs- oder Organisationsform zum demokratischen Zentralismus (SED 1963, S. 292).

Weiterhin kommt auch in Großschreibungen, dass über den deskriptiven Nennwert hinaus mit einer zusätzlichen Aufladung zu rechnen ist. Das Attribut wird in diesen Fällen zu einem festen Bestandteil des Schlagwortes. So wird der Internationaler Terrorismus (B 90/GRÜNE 2002, S. 18, $118,163)$ durch die Verwendung von Majuskeln als Schlagwort gekenn- 
zeichnet, während die Adjektive fundamentalistisch (B 90/GRÜNE 2002, S. 118), menschenverachtend (B 90/GRÜNE 2002, S. 119) und transnational (B 90/GRÜNE 2002, S. 150) als Attribute zum Substantiv Terrorismus verwendet werden und daher in Kleinbuchstaben erscheinen. Die SPD verwendet im Godesberger Programm den Ausdruck demokratischer Sozialismus zur Bezeichnung einer bestimmten (demokratischen) Form des Sozialismus; in ihrem Grundsatzprogramm von 1989 erscheint der Ausdruck Demokratischer Sozialismus aufgrund der Verwendung von Majuskeln als Schlagwort (SPD 1989, S. 5/8/10/12/13/19/25/52); daneben wird demokratisch als Attribut zu den Substantiven Element, Teilhabe, Rechte, Verfassung, Staat, Rechtsstaat, Sozialstaat, Aufbau, Volksbewegungen und sogar in Verbindung mit Sozialisten verwendet. Die FDP verwendet den Ausdruck des Sozialen Liberalismus (FDP 1971, S. 250) als Schlagwort und die CDU spricht von Sozialer Markwirtschaft (CDU 1971, S. 63).

Schlagworte in der politischen Sprachverwendung treten häufig im Verbund mit anderen Schlagworten auf; diese Tatsache bestätigt die zuvor formulierte Vermutung, dass die Elemente eines Wortschatzes auf komplexe Weise miteinander verflochten sind. So lässt sich hinsichtlich des Schlagworts Soziale Marktwirtschaft ein Schlagwortfeld oder "Schlagwortnetz ${ }^{\prime \prime 24}$ bilden, bestehend aus den in einem engen Zusammenhang stehenden Schlagwörtern Freiheit, Leistung, Angebot und Nachfrage, Wettbewerb, Kartell, Verbraucher, Unternehmer, Preisbildung, Tarifrecht, Tarifverträge und Tarifautonomie, Arbeitnehmer, Eigentum, Einzel- und Privateigentum, Markt, Partnerschaft und Wohlstand, die sich in den Parteiprogrammen nachweisen lassen. KLEIN (1998) untergliedert den Wissensrahmen (Frame) der Sozialen Marktwirtschaft nach (i) den Slots als Bezeichnungen des Systems, von Zentralwerten, Hauptbeteiligten, den Relationen zwischen den Hauptbeteiligten oder einem Hauptergebnis und (ii) den Fillern, die als aufwertende (von Befürwortern) bzw. abwertende (der Gegner) Schlagwörter die Slots füllen. Angewendet auf den Terminus Soziale Marktwirtschaft bedeutet das konkret:

,Freiheit' und ,Leistung' als Filler für den Slot Zentralwert, ,Wettbewerb' als Filler für den Slot Funktionsprinzip, ,Verbraucher', ,Unternehmer' und ,Arbeitnehmer' als Filler für den Slot Hauptbeteiligte, ,Markt' und ,(Sozial-)Partnerschaft' als Filler für den Slot Relationen zwischen den Beteiligten sowie ,Wohlstand' als Filler für den Slot Ergebnis. ${ }^{425}$

424 Klein 1998, S. 380

425 Klein 1998, S. 381 


\subsection{Vexierwörter}

Eine weitere spezielle Form von Schlagwörtern stellen „Vexierwörter“426 dar. Bestimmte Inhalte in politischen Auseinandersetzungen müssen sprachlich gefasst sein, damit sie vermittelt und diskutiert werden können. Eine bestimmte Formulierung kann jedoch aufgrund lexikalischer und syntaktischer Polysemien unterschiedliche Inhalte implizieren und Sachverhalte lassen sich unterschiedlich benennen. Diese Voraussetzungen können in der politischen Sprachverwendung dazu dienen, absichtlich begriffliche Ungenauigkeiten, d.h. Missverständnisse zu provozieren. Eine Kaschierstrategie ist der Gebrauch von Wörtern als Vexierwörter, d.h. ein Gebrauch, der beim Gros der Adressaten gezielt andere Vorstellungen weckt, als die Insider-Bedeutung des Wortes hergibt. Vexierwörter sind folglich

Wörter, die bewußt dazu gebraucht werden, Absichten zu verschleiern und Adressaten zu täuschen, indem sie mit einer anderen Bedeutung als der etablierten verwendet werden [...]. Solche Wörter verursachen Verständigungskonflikte oder Kommunikationskonflikte. ${ }^{427}$

Die Verwendung eines Wortes als Vexierwort setzt jedoch eine neue, von der alten (bisher etablierten) abweichende Bedeutungsfestlegung dieses Wortes voraus. So unterscheidet TEUBERT (1989) zwischen (i) der etablierten Bedeutung eines Wortes (Gebrauchsregeln, die gegenseitiges Verstehen und eine Verständigung möglich machen) und (ii) der normierten Bedeutung eines Wortes,

wenn Bedeutungen bewußt abweichend von der etablierten Bedeutung in einer Weise festgelegt und verwendet werden, daß sich mit ihnen gesellschaftliche Interessen durchsetzen lassen, solange es den Normierern gelingt, daß sich die Adressaten auf die solchermaßen geschaffenen Sprachzwänge einlassen. ${ }^{428}$

Die Theorie der Vexierwörter und deren Verwendung verdeutlicht auch die verbreitete anschauliche oben bereits erwähnte Metapher des Begriffe Besetzens, die in diesen Zusammenhang gehört und die dem ehemaligen Generalsekretär der CDU Kurt Biedenkopf zugeschrieben wird. Biedenkopf geht von einer „Revolution der Gesellschaft durch die Sprache“ aus und weiterhin davon, dass Regierungen durch die Besetzung von Begriffen regieren. Am 18. November 1973 auf dem 22. Bundesparteitag seiner Partei in Hamburg äußert sich Biedenkopf folgendermaßen:

426 Teubert, Wolfgang: „Politische Vexierwörter.“ In: Politische Semantik. Beiträge zur politischen Sprachverwendung. Hg. v. Josef Klein. Opladen 1989, S. 51

427 Teubert 1989, S. 52

428 Teubert 1989, S. 52 
Sprache, liebe Freunde, ist nicht nur ein Mittel der Kommunikation. Wie uns die Auseinandersetzung mit der Linken zeigt, ist Sprache auch ein wichtiges Mittel der Strategie. Was sich heute in unserem Lande vollzieht, ist eine Revolution neuer Art. Es ist die Revolution der Gesellschaft durch die Sprache. Die gewaltsame Besetzung der Zitadellen staatlicher Macht ist nicht länger Voraussetzung für eine revolutionäre Umwälzung der staatlichen Ordnung. Revolution findet heute auf eine andere Weise statt. Statt der Gebäude der Regierung werden die Begriffe besetzt, mit denen sie regiert, die Begriffe, mit denen wir unsere staatliche Ordnung, unsere Rechte und Pflichten und unsere Institutionen beschreiben. [...] Deshalb, meine Freunde, ist die Auseinandersetzung mit der politischen Sprache von so großer Bedeutung. Wir erleben heute eine Revolution, die sich nicht der Besetzung der Produktionsmittel, sondern der Besetzung der Begriffe bedient. Sie besetzt Begriffe und damit die Information in der freien Gesellschaft, indem sie die Medien besetzt, die Stätten also, in denen das wichtigste Produkt einer Freiheit hergestellt wird: die politische Information. ${ }^{429}$

Es geht beim Besetzen von Begriffen, beim Kampf um Begriffe ${ }^{430}$ oder beim Kampf um Wörter ${ }^{431}$ folglich um den Wettbewerb um Wörter, d.h. um einen semantischen Kampf sowie um das damit verbundene, auf die politische Kommunikation beschränkte Verfahren, einen „noch nicht allgemein bekannten Ausdruck zu besetzen und ihn durchzusetzen als ein neues Markenzeichen der Partei“432, d.h. „mit den eigenen Begriffen auch die eigenen Sehweisen öffentlich durchzusetzen bzw. durchsetzen zu wollen. " 433 Das Begriffe Besetzen hat weiterhin das Ziel, „daß man bestimmte allgemein verbindliche Grundgrundwerte begrifflich mit einer politischen Partei so verbindet, daß letztlich diese Partei für diesen Grundwert steht" ${ }^{434}$ und ist demzufolge ein „Kampf um den Sieg der eigenen Sprachregelung und damit auch der eigenen Ideenwelt, der eigenen Ideologie." ${ }^{435}$ Mit dieser Betrachtung geht die Ansicht der unter bestimmten Voraussetzungen stattfindenden Gewaltanwendung im Sprachgebrauch einher, was an den Begriffszuschreibungen

429 CDU 1973, S. 61

430 Geißler 1985, S. $222 \mathrm{ff}$.

431 Greiffenhagen 1980

432 Hermanns, Fritz: „,'Leistung' und ,Entfaltung'. Ein linguistischer Beitrag zur Interpretation des Ludwigshafener Grundsatzprogramms (1978) der CDU.“ In: Begriffe besetzen. Strategien des Sprachgebrauchs in der Politik. Hg. v. Frank Liedtke/Martin Wengeler/Karin Böke. Opladen 1991, S. 236

433 Burkhardt 2003, S. 360

434 Liedtke, Frank/Wengeler, Martin/Böke, Karin: Begriffe besetzen. Strategien des Sprachgebrauchs in der Politik. Opladen 1991, Vorwort S. 9

435 Bergsdorf 1978, S. 49 
(Un-)Freiheit $\leftrightarrow$ Kapitalismus und (Un-)Freiheit $\leftrightarrow$ Sozialismus deutlich wird:

Derartige erfolgreiche Begriffsbesetzungen können sich als strukturelle Gewalt auswirken, wenn die Selbstreferenz einer Gruppe, etwa als Sozialisten (im Sinne einer bestimmten politischen Theorie) absichtsvoll entwertet wird und auf eine andere Gruppe übertragen und trotz des Protests der Betroffenen daran festgehalten wird. Ziel ist die Verdrängung der etablierten (neutralen oder positiven) Bezeichnung Sozialdemokraten. ${ }^{436}$

Die Begriffsverwirrungen bestimmter Wörter (Vexierwörter) resultieren dabei aus ständig wiederholten Verwendung in abweichenden Kontexten. TEUBERT (1989) wertet die Wörter Subventionen und Klassenkampf als Vexierwörter, da beide Ausdrücke in verschiedenen Kontexten unterschiedliche begriffliche Inhalte tragen und dadurch in bestimmten Verwendungsweisen gebraucht und vom politischen Gegner "missbraucht" werden, oder weil beispielsweise der Ausdruck Klassenkampf eine begriffliche Verschiebung erfahren hat (vom früheren gesellschaftlichen Kampf zwischen zwei gegensätzlichen Klassen um die Entscheidungsgewalt in der Gesellschaft (a) über die Stigmatisierung (b) zum Kampf der Arbeitnehmer gegen die Unternehmer in der heutigen Verwendung). Die Verwendungsweise von Klassenkampf in Äußerung (a) lässt sich auch in den Programmen der KPD von 1918/19 sowie der SED von 1963 nachweisen, während die SPD im Godesberger Programm und im Jahre 1989 auf den Gebrauch dieses Ausdrucks verzichtet und durch den Ausdruck Kampf der Arbeiterbewegung ersetzt (c).

(61) (a) In den Aktionen für ihre unmittelbaren wirtschaftlichen und sozialen Interessen erwirbt die arbeitende Bevölkerung, durch die Tätigkeit einer marxistischen Partei vermittelt, Vertrauen in ihre eigene Kraft, Einsicht in die Notwendigkeit des Klassenkampfes und weitergehender politischer und gesellschaftlicher Veränderungen. (DKP 1969, S. 326)

(b) Soziale Gerechtigkeit wurde noch niemals durch sozialistischen Klassenkampf oder marxistische Revolutionen verwirklicht. (NPD 1973, S. 347)

(c) Solidarität hat die Arbeiterbewegung im Kampf für Freiheit und Gleichheit geprägt und ermutigt. (SPD 1989, S. 13)

Abschließend sei noch eine Bemerkung zur Betrachtung einiger Schlagwörter als Abstrakta angefügt. Die Bezeichnung Abstrakta ist in diesem

436 Zimmermann, Rüdiger: "Gewalt in der Sprache und durch Sprache." In: Wörter in der Politik. Analysen zur Lexemverwendung in der politischen Kommunikation. Hg. v. Hajo Diekmannshenke/Josef Klein. Opladen 1996, S. 118 
Zusammenhang nicht unproblematisch. Zwar sind bestimmte Ausdrücke in isolierter Betrachtung abstrakt und begrifflich unklar. Im Kontext des Parteiprogramms der SED ist beispielsweise der Ausdruck Planwirtschaft jedoch eindeutig determiniert. Auch Ausdrücke wie Freiheit, Demokratie oder Sozialismus sind zwar grundsätzlich abstrakt, dennoch bezeichnen sie Dinge, Sachverhalte oder Vorgänge der Wirklichkeit, genauer des gesellschaftlichen Miteinanders. Die Komplexität überträgt sich somit auf die Ausdrücke, die jeweils Teilbereiche davon bezeichnen. Kompliziert werden die Begriffe dann, wenn sie „Ordnungen bezeichnen, die der unmittelbaren Erfahrungswelt des Durchschnittsbürgers entrückt sind “437; in einem solchen Fall ergibt sich ein tatsächliches Verständigungsproblem, das jedoch vom jeweiligen Rezipientenkreis abhängt.

\subsubsection{Erzeugung von Sichtweisen}

Wenn in den folgenden Kapiteln von der Erzeugung von Sichtweisen die Rede ist, dann sind damit Zuschreibungen gemeint, die durch bestimmte Wortverwendungen und Formulierungen gemacht werden. Solche $\mathrm{Zu}-$ schreibungen ergeben sich häufig einerseits aus der Profilfunktion, die die jeweilige Partei veranlasst, die eigene Programmatik von der Programmatik anderer Parteien abzugrenzen sowie andererseits auch als Voraussetzung für die politische Werbung.

Die Zuschreibungen können daher sowohl positiver als auch negativer Prägung sein, wobei sich erstere zumeist auf den Produzenten der sprachlichen Handlung beziehen und letztere häufig explizit am politischen Gegner orientieren. Aus der Sicht des Produzenten einer sprachlichen Handlung geht es bei der Erzeugung von Sichtweisen folglich um Selbstaufwertung und Gegnerabwertung. Die Erzeugung von Sichtweisen als sprachliches Handlungsziel in der Politik stellt somit das deutlichste Kennzeichen „ideologischer Verbrämung“438 dar.

Die nachfolgend zitierten Textpassagen der KPD (a) und SED (b) verdeutlichen, wie Aufwertungen und Abwertungen mit Hilfe des Gegensatzes als Stilmittel erzeugt werden können.

(62) (a) Höchster Idealismus im Interesse der Allgemeinheit, straffste Selbstdisziplin, wahrer Bürgersinn der Massen sind für die sozialistische Gesellschaft die moralische Grundlage, wie Stumpfsinn, Egoismus und Korruption die moralische Grundlage der kapitalistischen Gesellschaft sind. (KPD 1918/19, S. 110) 
(b) So treffen in der Außenpolitik der beiden deutschen Staaten die zwei Linien der Weltpolitik aufeinander: Dem Kampf um Frieden und Abrüstung, der friedlichen Koexistenz und Völkerfreundschaft steht die imperialistische Politik der Kriegsvorbereitung und Kriegsdrohung, der Revanche, des Wettrüstens und des Neokolonialismus gegenüber. (SED 1963, S. 255)

Die Konzeption des oben zitierten Satzes der KPD ist eng an die Ideologie dieser Partei gebunden, da die Substantive mit positiver (tugendhafter) Nebenbedeutung wie Idealismus, Selbstdisziplin oder Bürgersinn (jeweils mit hervorhebenden und betonenden Attributen versehen) der sozialistischen Ideologie und die verachtenswerten Substantive mit negativer Nebenbedeutung wie Stumpfsinn, Egoismus oder Korruption der kapitalistischen Ideologie zugeschrieben werden. Bei dieser Gegenüberstellung innerhalb eines Satzes werden die Grundüberzeugungen (Sozialismus einerseits und Kapitalismus andererseits) von den Produzenten der sprachlichen Handlung (hier die Partei KPD als Verfasserkollektiv) bewertet und die Kontraste zwischen beiden durch diese Bewertung und die daraus resultierenden Zuschreibungen betont. Auch die SED stellt die Attribute friedlich und imperialistisch gegenüber; der Ausdruck Abrüstung auf der einen (eigenen) Seite findet seine negative Entsprechung im Ausdruck Wettrüsten, Frieden wird der Kriegsvorbereitung und der Kriegsdrohung gegenübergestellt und die negative Entsprechung zur (friedlichen) Koexistenz und zur Völkerfreundschaft lautet Neokolonialismus. Zu diesem Zusammenhang schreibt KLEIN (1998):

In den Auf- bzw. Abwertungsstrategien wird die jeweilige Position/Person entweder direkt bewertet, oder sie wird argumentativ oder assoziativ mit Sachverhalten von eindeutiger Wertigkeit verknüpft. Dabei besteht die Tendenz zu polarisierender Werte-Verteilung auf die eigene und die konkurrierende Position - meist ohne Zwischenwerte. 439

Dieser Untersuchungspunkt ist eng an die bereits behandelten Untersuchungsaspekte bzw. die behandelten Kapitel Gegnerbezug oder Anspruch auf Erfüllbarkeit sowie der Schlagwörter und der Metaphern gebunden, da es auch hier um die Erzeugung bzw. Übertragung bestimmter Wahrnehmungsmuster geht.

\subsection{Erzeugung positiver Sichtweisen/Euphemismen}

Insbesondere bei der Erzeugung von positiven Sichtweisen geht es um die "Wiederherstellung der getilgten Wahrheit mit semantischen Mitteln.“ ${ }^{440}$ Es geht dabei in der politischen Sprachverwendung um die „Ver-

439 Klein 1998, S. 378

440 Brecht, Bertolt: Gesammelte Werke. Band 20. Frankfurt/Main 1967, S. 191 
schleierung nach außen und/oder der Beschwichtigung und Verdrängung nach innen. " ${ }^{441}$, sowie um die Aufwertung der eigenen Position, indem diese hervorgehoben und die gegnerischen abgeschwächt oder schlichtweg vermieden werden. Die eigene Position wird in den Parteiprogrammen häufig mit positiven Werten gekoppelt, wie beispielsweise die wiederholte Erwähnung von Demokratie oder Wohlstand sowie die Grundwerte Freiheit und Gerechtigkeit. Auch durch das Berufen auf das Gemeinwohl werden eigene Ziele als uneigennützig ausgegeben.

Weiterhin werden mildernde oder täuschende Ausdrücke verwendet, die weniger positive Sachverhalte beschönigen; LEINFELLNER (1971) bezeichnet einen Euphemismus daher auch als „Deckwort“ bzw. als „Deckausdruck für soziale Tabus. " ${ }^{422}$ Charakteristisch sind solche Euphemismen insbesondere für die Sprache des NS-Regimes. Durch die Wortwahl werden somit unterschiedliche Perspektiven verliehen und subjektive Interessen wirksam.

Folgende Textstellen können als euphemistische Äußerungen betrachtet werden:

(63) (a) Ziel dieser Maßnahme ist nicht nur die Schaffung von mehr Erwerbsarbeitsplät-zen, sondern ausdrücklich auch die Verringerung der Einkommensunterschiede, also die Angleichung der Einkommen. (DIE FRAUEN 2002, S. 8)

(b) Friedenspolitik muß Machtkonflikte entschärfen, Interessenausgleich suchen, gemeinsame Interessen aufgreifen, dem Vormachstreben der Weltmächte durch regionale Zusammenschlüsse entgegenwirken und Gegensätze zwischen Systemen, Ideologien und Religionen im friedlichen Wettbewerb und in einer Kultur des politischen Streits austragen. Friedenspolitik muß die Vorherrschaft militärischer, bürokratischer und rüstungswirtschaftlicher Interessen brechen und Rüstungsproduktion in die Produktion ziviler Güter überführen. (SPD 1989, S. 15)

(c) Der Betrieb von Atomkraftwerken birgt immer ein Restrisiko, das zu einem fatalen Unfall führen kann, sei es durch menschliches Versagen oder einen gezielten Terroranschlag. (DIE FRAUEN 2002, S. 55)

Der Ausdruck Friedenspolitik in Äußerung (b) verdeutlicht, wie die Bildung eines Kompositums mit einem Hochwertwort wie Frieden als Komponente euphemistisch verwendet werden kann; Friedenspolitik kann insbesondere in der Textstelle der SPD einen militärischen Einsatz bedeuten, da nicht garantiert werden kann, dass die Überführung von Rüstungsproduktion in die Produktion ziviler Güter auf diplomatischem Wege

441 Burkhardt 1988, S. 342

${ }_{442}$ Leinfellner 1971, S. 69 
erreicht wird. Somit kann auch die Bombardierung einer vermeintlichen Waffenproduktionsanlage als für die Friedenspolitik notwendige Maßnahme eingestuft werden (wie etwa im August 1998 der Beschuss der Pharmafabrik al-Chifaa nahe der sudanesischen Hauptstadt Khartum durch die USA unter Bill Clinton).

Ebenso ist das Restrisiko in Äußerung (c) nichts anderes als ein Risiko. Durch die Verwendung des Ausdrucks Restrisiko soll zum Ausdruck gebracht werden, dass trotz aller erdenklicher Sicherheitsvorkehrungen Gefahrensituationen zwar eintreten können, jedoch mit geringer Wahrscheinlichkeit, da lediglich der Rest eines Risikos verbleibt. Der radioaktive Störfall von Tschernobyl mit den verheerenden Folgen zeigt allerdings, dass ein Restrisiko - verglichen mit dem Ausdruck Risiko - nicht weniger negative Auswirkungen für Betroffene nach sich zieht. Beide Ausdrücke beziehen sich daher als Referenz auf identische Sachverhalte, in der sprachlichen Realisierung Restrisiko jedoch auf beschönigende Weise. Aus diesem Grund schreiben die GRÜNEN in ihrem Grundsatzprogramm mit Verwendung von distanzierenden bzw. ironisierenden Anführungszeichen: „Die terroristischen Angriffe vom 11. September 2001 stellen den Begriff ,Restrisiko' in ein neues Licht. Ein Supergau wie Tschernobyl mit unermesslichen Folgen für Mensch und Natur kann durch noch so viel Sorgfalt nicht ausgeschlossen werden. Dieses Risiko dürfen wir nicht eingehen" (GRÜNE 2002, S. 30). Der Ausdruck Restrisi$k o$ kann damit als Euphemismus und dessen Verwendung als „lexikalische Kaschierstrategie "443 betrachtet werden, da negativ bewertete Eigenschaften des Bezeichneten abgeschwächt werden; die Kaschierstrategie dazu dient, Dritte über einen schlimmen Sachverhalt zu täuschen oder sie dient der moralischen Selbstentlastung.

Auch der Ausdruck Soziale Marktwirtschaft ist nach LEINFELLNER (1971) als Euphemismus zu betrachten, der aus der Regel resultiert, dass „,bei gegebenen pragmatischen Umständen versteckte semantisch-deskriptive Widersprüche euphemistische Funktion haben können. " ${ }^{444}$ Der Widerspruch des Ausdrucks Soziale Marktwirtschaft liegt in der Unvereinbarkeit von sozial einerseits und Marktwirtschaft andererseits, verstanden als freie Marktwirtschaft. Die Bezeichnung Soziale Marktwirtschaft beinhaltet somit den Widerspruch soziale freie Marktwirtschaft (eine freie Marktwirtschaft mit freiem und uneingeschränktem Wettbewerb kann nicht sozial sein) und könnte ebenso gut marktwirtschaftlicher Sozialismus lauten. Mit dem Ausdruck Soziale Marktwirtschaft werden jedoch aufgrund des Adjektivs sozial einerseits die finanziell schlechter situierten Teile der Gesellschaft angesprochen, andererseits assoziiert der Unternehmer mit der

$443 \quad$ Klein 1998, S. 384

444 Leinfellner 1971, S. 101 
Komponente Marktwirtschaft das Attribut frei und den damit einhergehenden uneingeschränkten Wettbewerb. So scheint es sich bei diesem Ausdruck um eine kalkuliert ambivalente Bezeichnung zu handeln, die möglichst alle Bevölkerungsgruppen zufrieden stellt und auf nahezu jeden Kontext anwendbar ist, unabhängig davon, „ob der Staat regulierend eingreift, Betriebe verstaatlicht, sozialen Wohnbau fördert, den Wohnbau dem Privatkapital überlässt, Riesenkonzerne gestattet. ${ }^{\prime 445}$ Auch KLEIN (1998) sieht den Terminus Soziale Marktwirtschaft als aufgewertete „feste Attributverknüpfung, wobei [...] primär die Attribute die Deontik bestimmen. ${ }^{446}$

\subsection{Erzeugung negativer Sichtweisen}

(64) (a) Die Kommunisten unterdrücken die Freiheit radikal. Sie vergewaltigen die Menschenrechte und das Selbstbestimmungsrecht der Persönlichkeit und der Völker. (Godesberger Programm 1959, S. 86)

(b) Wir müssen die Vergewaltigung der Natur aufgeben, um mit ihr zu überleben. (GRÜNE 1980, Kap. IV, 1)

(c) Statt nationaler Solidarität wird Klassenkampf gezüchtet. (NPD 1973, S. 343)

(d) Der in Selbstsucht, Bequemlichkeit und Feigheit entartete Liberalismus schützt und erhält die Freiheit der Menschen und Völker nicht. Der Marxismus vermag nur Funktionärsdiktaturen zu errichten und Völker und Menschen zu knechten. Beide Ideologien sind am Ende. Sie erstreben eine Gesellschaftsform, die Kulturvölker seit zwei Menschenaltern überwunden haben. (NPD 1973, S. 344)

Hinsichtlich des Verbs vergewaltigen in den Äußerungen (a) und (b) lassen sich zwei Bedeutungen formulieren:

1. jemanden „durch Anwendung, Androhung von Gewalt zum Geschlechtsverkehr zwingen" [...];

2. jemanden „auf gewaltsame Weise seinen Interessen, Wünschen unterwerfen. ${ }^{447}$

In beiden Äußerungen ist das Verb vergewaltigen gemäß der zweiten Bedeutung gebraucht, was insbesondere hinsichtlich der Verwendung in (b) mit einer Personifizierung der Natur einhergeht. Der Gebrauch von vergewaltigen bzw. Vergewaltigung nach der oben angegebenen ersten Bedeutung kann ebenso nachgewiesen werden: „Vergewaltigung oder sexualisierter Missbrauch sind besonders brutale Gewaltakte, keine Se-

\footnotetext{
445 Leinfellner 1971, S. 102/103

446 Klein 1998, S. 379

447 DUDEN 1999, S. 4214
} 
xualakte“ (DIE FRAUEN 2002, S.30); die feministische Partei DIE FRAUEN verwendet das Wort in ihrem Parteiprogramm in dieser Bedeutung insgesamt 11-mal, die GRÜNEN verwenden es in dieser Bedeutung in ihrem Grundsatzprogramm von 1980 insgesamt 2-mal, alle anderen untersuchten Parteiprogramme verzichten auf die Verwendung der Wörter vergewaltigen oder Vergewaltigung. In Äußerung (c) impliziert die Verwendung des Verbs züchten in Verbindung mit dem Ausdruck Klassenkampf, dass die einzige Handlungsabsicht darin liegt, den Klassenkampf herbeizuführen. Ein neutrales Verb in diesem Kontext könnte verursachen sein, was der Aussage eine völlig andere Färbung verleihen würde. Die Ausdrücke Funktionärsdiktatur und knechten in Äußerung (d) rufen ebenfalls eine negative Nebenbedeutung hervor, was weiterhin durch die schlechten Verhaltensweisen oder Untugenden Selbstsucht, Bequemlichkeit und Feigheit verstärkt wird. Offensichtlich ist die Absicht der NPD, den politischen Gegner zu diffamieren und zu delegitimieren sowie weiterhin den Rezipienten für die eigene Position zu vereinnahmen. Somit tritt insbesondere in Äußerung (d) die Ideologiegebundenheit offen zutage. STRAßNER (1987) erklärt diesen Sachverhalt mit dem für rechtsradikale Gruppierungen (wie die NPD oder die Deutsche Volksunion (DVU)) typischen Aufbau einer Volksfeind-Ideologie, deren demagogische Grundrichtung sich folgendermaßen zusammenfassen lässt:

Wir werden betrogen; Eine mächtige Verschwörung bedroht uns; Das Verhängnis steht unmittelbar bevor; Unsere Regierung ist korrupt; Kommunismus und Kapitalismus sind unsere Feinde; Der Feind ist anrüchig; Unser Volk darf nicht verdorben werden, [...]." ${ }^{448}$

Die Verwendung von Äußerungen nach der Volksfeind-Ideologie kann als populistisch und demagogisch interpretiert werden und hat neben der Diffamierung des politischen Gegners das Ziel oder den Zweck, von der eigenen Unvollkommenheit abzulenken und den Rezipienten zu vereinnahmen: „Ein äußeres Feindsymbol zu haben ist für jede ideologische Gruppierung, aber auch für jedes totalitäre und angeschlagene Regime höchst willkommen, weil es innen und außen anschaulich abgrenzt.." ${ }^{449}$

448 Straßner 1987, S. 54

449 Straßner 1987, S. 54 


\section{ERGEBNISSE UND ZUSAMMENFASSUNG}

Deutlich wurde bei der intensiven Betrachtung der Parteiprogramme aus linguistischer Perspektive vor allem, dass die Sprachverwendung in erster Linie zweckorientiert ist. Dieser Eindruck entsteht insbesondere bei der Analyse einzelner Argumentationen, er lässt sich jedoch auch hinsichtlich der Verwendung von Schlagwörtern und Metaphern bestätigen. Die Ursache dieser Zweckoptimierung liegt in den unterschiedlichen Funktionen begründet, die ein Parteiprogramm erfüllen soll (oder muss). Dabei ist - wie ausführlich dargelegt wurde - insbesondere zwischen Wahl- und Grundsatzprogramm zu differenzieren.

Deutlich wurde weiterhin, dass die Orientierung am Rezipienten neben dem Aspekt der Ideologie als der einflussreichste Faktor bei der Konzeption eines Parteiprogramms betrachtet werden kann. Auf der Suche nach Wählerstimmen läuft eine Partei mit ihrem jeweiligen Parteiprogramm permanent Gefahr, die identifikatorische Funktion nicht mehr erfüllen zu können, da möglichst viele gesellschaftliche Gruppierungen erreicht werden sollen, im schlimmsten Fall jedoch keine Gesellschaftsschicht sich mehr direkt angesprochen und mit der Partei verbunden fühlt. Diese Tatsache findet sich insbesondere bei den Grundsatzprogrammen der beiden großen Volksparteien CDU und SPD bestätigt. Randparteien wie die PDS, DIE FRAUEN oder auch DIE GRÜNEN sind von dieser Gefahr weit weniger betroffen, jedoch kann es auch bei diesen Parteien passieren, dass sie aufgrund ihrer Entwicklung die eigentliche Parteibasis verlieren.

Die Ausführung oben zeigt ein Problem auf, dass sich durch die gesamte Untersuchung zieht. Wie in der Einleitung angekündigt, stand bei dieser Untersuchung die Inhaltsvermittlung im Vordergrund; dabei sollten die spezifischen sprachlichen Handlungsmuster beleuchtet, erörtert und verglichen werden, mit denen die Inhaltsvermittlung überhaupt erst ermöglicht und durchgeführt wird. Die Trennung von den jeweiligen politischen Inhalten fiel dabei schwer und war stellenweise kaum realisierbar. Diese Problematik lässt sich auch in der Forschungsliteratur erkennen. Zur politischen Sprachverwendung existiert eine Fülle an Literatur. Die Auswahl fällt dabei zwar schwer, jedoch bezieht sich viel Literatur auf die gesprochene Sprache in der Politik (Reden, Bundestagsdebatten, Parlamentsreden, Pressekonferenzen, Talkshows), und weniger auf Parteiprogramme als schriftlich fixierte Texte. Eine Vielzahl von politologischen Titeln bezieht sich auf die unterschiedlichen Parteien, deren Inhalte sowie deren Entwicklung, was insbesondere beim Godesberger Programm mit dessen epochaler Wirkung sowohl auf die SPD als Partei, als auch auf die Wählerschaft deutlich wurde. Literatur dieser 
Art ist für die Einbettung in den historischen Hintergrund dienlich, für die linguistischen Themenkomplexe dieser Untersuchung erschien sie bedingt gewinnbringend und wurde nur ergänzend hinzugezogen.

Auch der Vergleich des SED-Parteiprogramms mit den Bekenntnisschriften konnte nur durchgeführt werden, weil der Untersuchungsschwerpunkt in dieser Arbeit in der Linguistik liegt. Daher standen in diesem Kapitel lediglich die formalen Analogien zur Diskussion; Parallelen sind auf politologischer Ebene abwegig.

Auf einige Bereiche musste im Rahmen dieser Arbeit jedoch verzichtet werden. Darunter fällt auch das umfangreiche Forschungsgebiet Sprache des Nationalsozialismus, das in dieser Untersuchung nur ansatzweise betreten werden konnte, da das untersuchte Parteiprogramm der NSDAP von 1920 (noch) keine Wörter beinhaltet, die charakteristisch für die ideologische Verbrämung sind. Hinzuweisen ist an dieser Stelle auf das Handbuch Vokabular des Nationalsozialismus von SCHMITZ-BERNING450 sowie auf das Sprachwörterbuch im Nationalsozialismus von MÜLLER ${ }^{451}$. Unter Zuhilfenahme dieser beiden Werke ließe sich auch das Vokabular des NPD-Parteiprogramms auf mögliche nationalsozialistische oder rechtsextreme Ausdrücke überprüfen.

Auch auf eine in der Einleitung angekündigte systemlinguistische Analyse musste im Rahmen dieser Untersuchung verzichtet werden. Sicherlich ergebnisreich ließen sich die Betrachtungen der Wahlparolen, des attributiven Stils, der rhetorischen Fragen, der Appositionen und der Ausklammerungen als Teilbereiche der Syntax besprechen, die charakteristisch für die Textsorte Parteiprogramm sind.

Die Betrachtung möglicher Archaismen machte deutlich, dass den älteren und kämpferischen Parteiprogrammen dieser Untersuchung ein (unbeabsichtigtes) Pathos verliehen wird, wenn diese nach heutigen Gesichtspunkten bzw. mit heutiger Sprachauffassung rezipiert werden. Bei altertümlich anmutenden Kandidaten müssen daher die Herkunft und das Altersprofil berücksichtigt werden. Hier zeigt sich erneut, dass der historische Kontext bei der Darstellung der sprachlichen Mittel und der Interpretation der Inhalte nicht vernachlässigt werden darf: So ist die metaphorische Komponente bei dem von der KPD (1918/19) verwendeten Ausdruck Blutmeer (S. 107) vor dem Hintergrund des Ersten Weltkriegs von weitaus geringerer Bedeutung als bei heutiger Interpretation, und auch der Ausdruck Weltbrand (S. 107) sowie die Wendung Millionen auf

450 Schmitz-Berning, Cornelia: Vokabular des Nationalsozialismus. Berlin/New York 2000.

451 Müller, Senya: Sprachwörterbuch im Nationalsozialismus. Die ideologische Beeinflussung von Duden, Sprach-Brockhaus und anderen Nachschlagewerken während des ,Dritten Reichs'. Stuttgart 1994. 
Millionen dampfender Menschenopfer (S. 107) wirkt weniger überspitzt. Die umfangreiche Einbettung des Godesberger Programm in den historischen Kontext der Bundesrepublik Deutschland der späten 1950er Jahren (vgl. Kapitel 1.2.1.4.1) kann auf diese Weise begründet werden.

Die Betrachtung der Schlagwörter und Metaphern nimmt jeweils einen großen Teil in der Untersuchung in Anspruch. Der Analyse der Parteiprogramme war insbesondere die Differenzierung der Schlagwörter dienlich, und die Untergliederung nach Fahnen- und Stigmawörtern bzw. Miranda und Anti-Miranda verdeutlichte den signifikanten Einfluss der Ideologie auf den unterschiedlichen Gebrauch einzelner Ausdrücke. Daher sollte die Betrachtung der Parteiprogramme über eine blanke Beschreibung hinausreichen und insbesondere in den Kapiteln Sonderformen politischer Sprachverwendung deutlich werden, dass von den Parteiprogrammen unterschiedliche Signale an die jeweilige Rezipientengruppe ausgehen und auf diese Weise verschiedene Lesarten zugelassen werden. 



\section{BibLIOGRAPHIE}

\subsection{Primärliteratur}

Berthold, Lothar/Diehl, Ernst (Hrsg.): Revolutionäre deutsche Parteiprogramme. Vom kommunistischen Manifest zum Programm des Sozialismus. Berlin ${ }^{3} 1967$.

Hergt, Siegfried (Hrsg.): Parteiprogramme. SPD, CDU, CSU, FDP, DKP, NPD. Eine Dokumentation der Grundsatzprogramme und aktueller politischer Ziele. Opladen ${ }^{7} 1975$.

Hintze, Peter: Die CDU-Parteiprogramme. Eine Dokumentation der Ziele und Aufgaben. Bonn 1995.

Mommsen, Wilhelm (Hrsg.): Deutsche Parteiprogramme. München 1960.

Rosenberg, Alfred (Hrsg.): Das Parteiprogramm. Wesen, Grundsätze und Ziele der NSDAP. München ${ }^{24} 1942$.

SPD-Parteivorstand (Hrsg.): Grundsatzprogramm der Sozialdemokratischen Partei Deutschlands. Berlin 1998.

\subsection{Sekundärliteratur}

Anglizismen-Wörterbuch. Der Einfluß des Englischen auf den deutschen Wortschatz nach 1945. 3 Bände. Berlin/New York 1993.

Bachem, Rolf: Einführung in die Analyse politischer Texte. München 1979.

Bergsdorf, Wolfgang: Politik und Sprache. München 1978.

Bergsdorf, Wolfgang: Herrschaft und Sprache. Studie zur politischen Terminologie der Bundesrepublik Deutschland. Pfullingen 1983.

Böke, Karin/Liedtke, Frank/Wengeler, Martin: Politische Leitvokabeln in der Adenauer-Ära. Berlin/New York 1996.

Brecht, Bertolt: Gesammelte Werke. Band 20. Frankfurt/Main 1967.

Brinker, Klaus: „Aspekte der Textlinguistik.“ In: Germanistische Linguistik (106-107). Hg. v. Klaus Brinker. Hildesheim/Zürich/New York 1991, S. 7-17.

Brinker, Klaus: Linguistische Textanalyse. Eine Einführung in Grundbegriffe und Methoden. Berlin ${ }^{5} 2001$.

Brockhaus. Die Enzyklopädie. 24 Bände. Leipzig/Mannheim 201998. 
Brockhaus/Wahrig - Deutsches Wörterbuch. 6 Bände. Hg. v. Gerhard Wahrig/Hildegard Krämer/Harald Zimmermann. Wiesbaden 1982.

Bundestagswahl 1980. Die Parteien und ihre Programme. In: Informationen zur politischen Bildung 185. Hg. v. Bundeszentrale für politische Bildung. Bonn 1980.

Bühler, Karl: Sprachtheorie. Die Darstellungsfunktion der Sprache. Stuttgart ${ }^{3} 1999$.

Burger, Harald/Buhofer, Annelies/Sialm, Ambros: Handbuch der Phraseologie. Berlin/New York 1982.

Burger, Harald: „Besondere Formen lexikalischer Einheiten I: Phraseologismen." In: Lexikologie. Ein internationales Handbuch zur Natur und Struktur von Wörtern und Wortschätzen (1. Halbband). Hg. v. D. Alan Cruse/Franz Hundsnurscher/Michael Job/Peter Rolf Lutzeier. Berlin/New York 2002, S. 392-401.

Burger, Harald: Phraseologie. Eine Einführung am Beispiel des Deutschen. Berlin 22003.

Burkhardt, Armin: „Sprache in der Politik. Linguistische Begriffe und Methoden." In: Englisch-Amerikanische Studien 10 (Heft 3, 4). Münster 1988, S. 333-358.

Burkhardt, Armin: „Politolinguistik - Versuch einer Ortsbestimmung.“ In: Sprachstrategien und Dialogblockaden. Linguistische und politikwissenschaftliche Studien zur politischen Kommunikation. Hg. v. Josef Klein/Hajo Dieckmannshenke. Berlin/New York 1996, S. $75-100$.

Burkhardt, Armin: Das Parlament und seine Sprache. Studien zur Theorie und Geschichte parlamentarischer Kommunikation. Tübingen 2003.

CDU (Hrsg.): 22. Bundesparteitag der Christlich Demokratischen Union Deutschlands (18.-20. November 1973). Niederschrift. Hamburg 1973.

Czerwick, Edwin: „Politikverdrossenheit - politische Selbstreferenz und die ,Stimme des Volkes'." In: Sprachstrategien und Dialogblockaden. Linguistische und politikwissenschaftliche Studien zur politischen Kommunikation. Hg. v. Josef Klein/Hajo Dieckmannshenke. Berlin/New York 1996, S. 49-72.

Der kleine Katechismus D. Mart. Lutheri für die gemeine Pfarrherrn und Prediger. Abschnitt Die zehen Gebot, wie sie ein Hausvater seinem Gesinde einfältiglich furhalten soll. In: Die Bekenntnisschriften der evangelisch-lutherischen Kirche. Hg. vom Deutschen Evangelischen Kirchenausschuss im Gedenkjahr der Augsburgischen Konfession 1930. Göttingen ${ }^{3} 1956$, S. 507-512. 
Dieckmann, Walther: Politische Sprache - Politische Kommunikation. Vorträge - Aufsätze - Entwürfe. Heidelberg 1981.

Dieckmann, Walther: Sprache in der Politik. Einführung in die Pragmatik und Semantik der politischen Sprache. Heidelberg 1969.

Dieckmannshenke, Hajo/Klein, Josef: Wörter in der Politik. Analysen zur Lexemverwendung in der politischen Kommunikation. Opladen 1996.

DUDEN. Das große Wörterbuch der deutschen Sprache. 10 Bände. Mannheim/Leipzig/Wien/Zürich ${ }^{3} 1999$.

Edelman, Murray: Politik als Ritual. Die symbolische Funktion staatlicher Institutionen und politischen Handelns. Frankfurt/Main 1976.

Eisenberg, Peter: Grundriß der deutschen Grammatik. Stuttgart/Weimar 31994.

Elspaß, Stephan: „Phraseologie im deutschen Parlamentarismus. Zu historischen Entwicklungen im Sprachverhalten politischer Redner." In: Sprache des deutschen Parlamentarismus. Hg. v. Armin Burkhardt/Kornelia Pape. Wiesbaden 2000, S. 261-287.

Elspaß, Stephan: Phraseologie in der politischen Rede. Zur Verwendung von Phraseologismen in ausgewählten Bundestagsdebatten. Opladen/Wiesbaden 1998.

Enkvist, Nils Erik: „,Versuche zu einer Bestimmung des Sprachstils: Ein Essay in angewandter Sprachwissenschaft." In: Linguistik und Stil. Hg. v. John Spencer. Heidelberg 1972, S. 8-54.

Eppler, Erhard: Grundwerte für ein neues Godesberger Programm. Hamburg 1984.

Eroms, Hans-Werner: „Zur Analyse politischer Sprache.“ In: Linguistik und Didaktik 17. München 1974, S. 1-16.

Finegan, Edward: Language - Its Structure and Use. Fort Worth ${ }^{3} 1999$.

Fleischer, Wolfgang: Phraseologie der deutschen Gegenwartssprache. Tübingen ${ }^{2} 1997$.

Fleischer, Wolfgang/Michel, Georg/Starke, Günter: Stilistik der deutschen Gegenwartssprache. Frankfurt/Main ${ }^{2} 1996$.

Flohr, Heiner: Parteiprogramme in der Demokratie. Ein Beitrag zur Theorie der rationalen Politik. Göttingen 1968.

Geißler, Heiner: „Sprache und Politik. Kampf um Begriffe.“ In: Germanistik - Forschungsstand und Perspektiven. Vorträge des Deutschen Germanistentages 1984 (Teil 1). Hg. v. Georg Stötzel. Berlin/New York 1985, S. 222-230. 
Girnth, Heiko: Sprache und Sprachverwendung in der Politik. Eine Einführung in die linguistische Analyse öffentlich-politischer Kommunikation. Tübingen 2002.

Gläser, Rosemarie: „Die Kategorie ,Funktionalstil' in soziolinguistischer Sicht." In: Zeitschrift für Phonetik, Sprachwissenschaft und Kommunikationsforschung (Band 27). Berlin 1974, S. 487-496.

Gloning, Thomas: „Praktische Semantik und Linguistische Kommunikationsanalyse." In: Handbuch der Dialoganalyse. Hg. v. Gerd Fritz/Franz Hundsnurscher. Tübingen 1994, S. 113-129.

Gloning, Thomas: Organisation und Entwicklung historischer Wortschätze. Lexikologische Konzeption und exemplarische Untersuchungen zum deutschen Wortschatz um 1600. Tübingen 2003.

Greiffenhagen, Martin: Kampf um Wörter? Politische Begriffe im Meinungsstreit. München/Wien 1980.

Grewenig, Adi: Inszenierte Information. Politik und strategische Kommunikation in den Medien. Opladen 1993.

Grice, H. Paul: „Logik und Konversation.“ In: Handlung, Kommunikation, Bedeutung. Hg. v. Georg Meggle. Frankfurt 1979, S. 243-265.

Grundrechte. In: Informationen zur politischen Bildung 239. Hg. v. Bundeszentrale für politische Bildung. Bonn 1993.

Grünert, Horst: „Politische Geschichte und Sprachgeschichte. Überlegungen zum Zusammenhang von Politik und Sprachgebrauch in Geschichte und Gegenwart. “In: Sprache und Literatur in Wissenschaft und Unterricht (Band 53). München/Paderborn 1983, S. 43-58.

Heringer, Hans Jürgen/Öhlschläger, Günther/Strecker, Bruno/Wimmer, Rainer: Einführung in die Praktische Semantik. Heidelberg 1977.

Heringer, Hans Jürgen: „Sprachspiele und ihre Geschichte.“ In: Einführung in die Praktische Semantik. Hg. v. Hans Jürgen Heringer/Günther Öhlschläger/Bruno Strecker/Rainer Wimmer. Heidelberg 1977, S. 60-85.

Heringer, Hans Jürgen: ,Ich gebe Ihnen mein Ehrenwort.' Politik - Sprache - Moral. München 1990.

Hermanns, Fritz: „Deontische Tautologien.“ In: Politische Semantik. Beiträge zur politischen Sprachverwendung. Hg. v. Josef Klein. Opladen 1989, S. 69-149.

Hermanns, Fritz: „,Leistung' und ,Entfaltung'. Ein linguistischer Beitrag zur Interpretation des Ludwigshafener Grundsatzprogramms (1978) der CDU.“ In: Begriffe besetzen. Strategien des Sprach- 
gebrauchs in der Politik. Hg. v. Frank Liedtke/Martin Wengeler/Karin Böke. Opladen 1991, S. 230-257.

Herrgen, Joachim: Die Sprache der Mainzer Republik (1792/93). Historisch-semantische Untersuchungen zur politischen Kommunikation. Tübingen 2000.

Holly, Werner: Politikersprache. Inszenierungen und Rollenkonflikte im informellen Sprachhandeln eines Bundestagsabgeordneten. Berlin/New York 1990.

Holly, Werner: „Hier spricht der Zuschauer. Ein neuer methodischer Ansatz in der sprachwissenschaftlichen Erforschung politischer Fernsehkommunikation." In: Sprachstrategien und Dialogblockaden. Linguistische und politikwissenschaftliche Studien zur politischen Kommunikation. Hg. v. Josef Klein/Hajo Dieckmannshenke. Berlin/New York 1996, S. 101-121.

Hundsnurscher, Franz: „Dialog-Typologie.“ In: Handbuch der Dialoganalyse. Hg. v. Gerd Fritz/Franz Hundsnurscher. Tübingen 1994, S. 203-238.

Ickler, Theodor: „Zur Semantik des politischen Schlagwortes (und anderer Wörter)." In: Sprache und Literatur in Wissenschaft und Unterricht (Band 65). München/Paderborn 1990, S. 11-26.

Institut für Marxistische Studien und Forschungen e.V. (IMSF) (Hrsg.): SPD wohin? Zur Analyse und Kritik des SPD-

Grundsatzprogrammentwurfs. Informationsbericht Nr. 44. Frankfurt/Main 1987.

Jäger, Siegfried: „Gesellschaftsveränderungen über Sprache.“ In: Soziolinguistik. Ein internationales Handbuch zur Wissenschaft von Sprache und Gesellschaft. Zweiter Halbband. Hg. v. Ulrich Ammon/Norbert Dittmar/Klaus J. Mattheier. Berlin/New York 1988, S. 1789-1796.

Januschek, Franz: „Anspielungen und Distanzierungen im populistischen Mediendiskurs am Beispiel des österreichischen Politikers Jörg Haider." In: Inszenierte Information. Politik und strategische Kommunikation in den Medien. Hg. v. Adi Grewenig. Opladen 1993, S. 117-142.

Jarren, Otfried/Sarcinelli, Ulrich/Saxer, Ulrich (Hrsg.): Politische Kommunikation in der demokratischen Gesellschaft. Ein Handbuch mit Lexikonteil. Wiesbaden 1998.

Kaack, Heino: Geschichte und Struktur des deutschen Parteiensystems. Opladen 1971.

Kammer, Hilde/Bartsch, Elisabet: Nationalsozialismus. Begriffe aus der Zeit der Gewaltherrschaft 1933-1945. Reinbek 1992. 
Kayser, Wolfgang: Das sprachliche Kunstwerk. Eine Einführung in die Literaturwissenschaft. Tübingen/Basel 201992.

Kienpointner, Manfred: Argumentationsanalyse. Innsbruck 1983.

Kilian, Jörg: „,Sprache in der Politik. Ein einführender Überblick.“ In: Praxis Deutsch (Band 21). Seelze 1994, S. 4-10.

Kindt, Walther: „Argumentationskultur in Bundestagsreden - illustriert am Bespiel einer Debatte vom 2.4.1998.“ In: Sprache des deutschen Parlamentarismus. Hg. v. Armin Burkhardt/Kornelia Pape. Wiesbaden 2000, S. 319-335.

Klaus, Georg: Sprache der Politik. Berlin (Ost) 1971.

Klein, Josef: Die konklusiven Sprechhandlungen.: Studien zur Pragmatik, SEMANTIK, Syntax und Lexik von BEGRÜNDEN, ERKLÄRENWARUM, FOLGERN und RECHTFERTIGEN. Tübingen 1987.

Klein, Josef: „Wortschatz, Wortkampf, Wortfelder in der Politik.“ In: Politische Semantik. Beiträge zur politischen Sprachverwendung. Hg. v. Josef Klein. Opladen 1989, S. 3-50.

Klein, Josef: „Politische Textsorten.“ In: Germanistische Linguistik (106107). Hg. v. Klaus Brinker. Hildesheim/Zürich/New York 1991a, S. 245-278.

Klein, Josef: „Kann man ,Begriffe besetzen'? Zur linguistischen Differenzierung einer plakativen politischen Metapher." In: Begriffe besetzen. Strategien des Sprachgebrauchs in der Politik. Hg. v. Frank Liedtke/Martin Wengeler/Karin Böke. Opladen 1991b, S. 44-69.

Klein, Josef/Dieckmannshenke, Hajo: Sprachstrategien und Dialogblockaden. Linguistische und politikwissenschaftliche Studien zur politischen Kommunikation. Berlin/New York 1996.

Klein, Josef: „Insider-Lesarten. Einige Regeln zur latenten Fachkommunikation in Parteiprogrammen." In: Sprachstrategien und Dialogblockaden. Linguistische und politikwissenschaftliche Studien zur politischen Kommunikation. Hg. v. Josef Klein/Hajo Dieckmannshenke. Berlin/New York 1996a, S. 201-209.

Klein, Josef: „Dialogblockaden. Dysfunktionale Wirkungen von Sprachstrategien auf dem Markt der politischen Kommunikation." In: Sprachstrategien und Dialogblockaden. Linguistische und politikwissenschaftliche Studien zur politischen Kommunikation. Hg. v. Josef Klein/Hajo Dieckmannshenke. Berlin/New York 1996b, S. 329.

Klein, Josef: „Politische Kommunikation als Sprachstrategie.“ In: Politische Kommunikation in der demokratischen Gesellschaft. Ein 
Handbuch mit Lexikonteil. Hg. v. Otfried Jarren/Ulrich Sarcinelli/Ulrich Saxer. Wiesbaden 1998, S. 376-395.

Lakoff, George/Johnson, Mark: Metaphors we live by. Chicago/London 1980.

Langenscheidts Handwörterbuch Englisch. Berlin/München ${ }^{2} 1996$.

Leinfellner, Elisabeth: Der Euphemismus in der politischen Sprache. Berlin 1971.

Liedtke, Frank/Wengeler, Martin/Böke, Karin: Begriffe besetzen. Strategien des Sprachgebrauchs in der Politik. Opladen 1991, S. 9

Link, Elisabeth: „,Fremdwörter - Der Deutschen liebste schwere Wörter?" In: Deutsche Sprache. Zeitschrift für Theorie, Praxis und Dokumentation. Berlin 1983, S. 47-77.

Linke, Angelika/Nussbaumer, Markus/Portmann, Paul R.: Studienbuch Linguistik. Tübingen ${ }^{3} 1996$.

Lübbe, Hermann: „Der Streit um Worte. Sprache und Politik.“ In: Holzfeuer im hölzernen Ofen. Aufsätze zur politischen Sprachkritik. Hg. v. Hans Jürgen Heringer. Tübingen 1982, S. 48-69.

Marx-Nordin, Signe: Studien zum Stil deutschsprachiger Parteiprogramme. Ein Beitrag zu einer syntaxorientierten Stilistik. Göteborg 1979.

Massenmedien. In: Informationen zur politischen Bildung 260. Hg. v. Bundeszentrale für politische Bildung. Bonn 1998.

Miller, Susanne: Die SPD vor und nach Godesberg. Bonn 1975.

Müller, Helmut M.: Schlaglichter der deutschen Geschichte. Mannheim 31996.

Müller, Senya: Sprachwörterbuch im Nationalsozialismus. Die ideologische Beeinflussung von Duden, Sprach-Brockhaus und anderen Nachschlagewerken während des ,Dritten Reichs'. Stuttgart 1994.

Offe, Claus: „Editorial.“ In: Murray Edelman: Politik als Ritual. Die symbolische Funktion staatlicher Institutionen und politischen Handelns. Frankfurt/Main 1976, VII-X.

Öhlschläger, Günther: „Argumentieren.“ In: Einführung in die Praktische Semantik. Hg. v. Hans Jürgen Heringer/Günther Öhlschläger/Bruno Strecker/Rainer Wimmer. Heidelberg 1977, S. 251-274.

OXFORD Advanced Learner's Dictionary of Current English. Hg. v. Sally Wehmeier. Oxford ${ }^{6} 2000$. 
Parlamentarische Demokratie I. In: Informationen zur politischen Bildung 227. Hg. v. Bundeszentrale für politische Bildung. Bonn 32000 .

Polenz, Peter von: Deutsche Satzsemantik. Grundbegriffe des Zwischenden-Zeilen-Lesens. Berlin/New York 1985.

Politische Ideologien. In: Informationen zur politischen Bildung 212. Hg. v. Bundeszentrale für politische Bildung. Bonn ${ }^{2} 1990$.

PONS - Deutsche Idiomatik. Die deutschen Redewendungen im Kontext. Hg. v. Hans Schemann. Stuttgart/Dresden 1993.

Sandig, Barbara: Stilistik der deutschen Sprache. Berlin/New York 1986.

Sartori, Giovanni: „Constitutionalism: A Preliminary Discussion.“ In: The American Political Science Review (APSR). Volume 56. Menasha, Wisconsin 1962, S. 853-864.

Saussure, Ferdinand de: Grundfragen der allgemeinen Sprachwissenschaft. Berlin/New York ${ }^{3} 2001$.

Schäffner, Christina: „Europapolitische Metaphorik in England und Deutschland." In: Sprachstrategien und Dialogblockaden. Linguistische und politikwissenschaftliche Studien zur politischen Kommunikation. Hg. v. Josef Klein/Hajo Dieckmannshenke. Berlin/New York 1996, S. 151-163.

Schippan, Thea: Lexikologie der deutschen Gegenwartssprache. Tübingen 22002.

Schmidt, Wilhelm: „Zur Ideologiegebundenheit der politischen Lexik." In: Zeitschrift für Phonetik, Sprachwissenschaft und Kommunikationsforschung (Band 22). Berlin 1969, S. 255-271.

Schmitz-Berning, Cornelia: Vokabular des Nationalsozialismus. Berlin/New York 2000.

Schönbohm, Wulf: „Funktion, Entstehung und Sprache von Parteiprogrammen." In: Aus Politik und Zeitgeschichte B 34-35. Hg. v. Bundeszentrale für politische Bildung. Bonn 1974, S. 17-37.

Schumacher, Kurt: „Die Sozialdemokratie und die Verteidigung Deutschlands." Gehalten am 23.08.1950 in Bonn. In: Kurt Schumacher. Reden - Schriften - Korrespondenzen 1945-1952. Hg. v. Willy Albrecht. Berlin/Bonn 1985, S. 828-835.

Searle, John R.: Ausdruck und Bedeutung. Untersuchungen zur Sprechakttheorie. Frankfurt/Main 1982.

Sowinski, Bernhard: Deutsche Stilistik. Beobachtungen zur Sprachverwendung und Sprachgestaltung im Deutschen. Frankfurt 1973. 
Sowinski, Bernhard: Werbeanzeigen und Werbesendungen. München 1979.

Spencer, John (Hrsg.): Linguistik und Stil. Heidelberg 1972.

Spencer, John und Gregory, Michael: „Eine Stellungnahme zum Sprachstil." In: Linguistik und Stil. Hg. v. John Spencer. Heidelberg 1972, S. 55-94.

Stalnaker, Robert C.: „Pragmatics.“ In: Semantics of Natural Language. Hg. v. Donald Davidson und Gilbert Harman. Dordrecht 1972, S. 380-397.

Stötzel, Georg/Wengeler, Martin: Kontroverse Begriffe. Geschichte des öffentlichen Sprachgebrauchs in der Bundesrepublik Deutschland. Berlin/New York 1995.

Straßner, Erich: Ideologie - SPRACHE - Politik. Grundfragen des Zusammenhangs. Tübingen 1987.

Strauß, Gerhard: „Sprachspiele, kommunikative Verfahren und Texte in der Politik. Versuch einer Textsortenspezifik." In: Der politische Wortschatz. Zur Kommunikations- und Textsortenspezifik. Hg. v. Gerhard Strauß. Tübingen 1986a, S. 2-66.

Strauß, Gerhard/Zifonun, Gisela: „Formen der Ideologiegebundenheit: Versuch einer Typologie der gesellschaftspolitischen Lexik." In: Der politische Wortschatz. Zur Kommunikations- und Textsortenspezifik. Hg. v. Gerhard Strauß. Tübingen 1986b, S. 67-147.

Strauß, Gerhard: „Schwere Wörter in der Politik.“ In: Der politische Wortschatz. Zur Kommunikations- und Textsortenspezifik. Hg. v. Gerhard Strauß. Tübingen 1986c, S. 149-280.

Strauß, Gerhard/Haß, Ulrike/Harras, Gisela: Brisante Wörter - von Agitation bis Zeitgeist. Ein Lexikon zum öffentlichen Sprachgebrauch. Berlin/New York 1989.

Teubert, Wolfgang: „Politische Vexierwörter.“ In: Politische Semantik. Beiträge zur politischen Sprachverwendung. Hg. v. Josef Klein. Opladen 1989, S. 51-68.

Vater, Heinz: Einführung in die Sprachwissenschaft. München ${ }^{4} 2002$.

Verfassung des Landes Hessen und Grundgesetz für die Bundesrepublik Deutschland. Mit einer Einführung und einem Anhang: Allgemeine Erklärung der Menschenrechte - Konvention zum Schutze der Menschenrechte und Grundfreiheiten (Auszug) - Charta der Vereinten Nationen. Hg. v. Georg August Zinn und Erwin Stein. Bad Homburg vor der Höhe ${ }^{421989, ~ S . ~ 170-228 . ~}$ 
Verfassung des Landes Hessen und Grundgesetz für die Bundesrepublik Deutschland. Mit einer Einführung und einem Anhang: Allgemeine Erklärung der Menschenrechte - Konvention zum Schutze der Menschenrechte und Grundfreiheiten (Auszug) - Charta der Vereinten Nationen. Hg. v. Georg August Zinn und Erwin Stein. Bad Homburg vor der Höhe ${ }^{451992, ~ S . ~ 162-220 . ~}$

Verhandlungen des Deutschen Bundestages. Stenographische Berichte. 1. Wahlperiode 1949. Bonn 1950.

Volmert, Johannes (Hrsg.): Grundkurs Sprachwissenschaft. Eine Einführung in die Sprachwissenschaft für Lehramtsstudiengänge. München ${ }^{3} 1999$.

Volmert, Johannes: „'While the world prayed for peace, Saddam prepared for war.' Bushs Fernsehrede zur Eröffnung des Bombenkriegs gegen den Irak." In: Inszenierte Information. Politik und strategische Kommunikation in den Medien. Hg. v. Adi Grewenig. Opladen 1993, S. 198-230.

Weber, Max: „Politik als Beruf.“ In: Politisches Denken im 20. Jahrhundert. Ein Lesebuch. Hg. v. Herfried Münkler. München 21997, S. 22-34.

Weber, Max: „Soziologische Grundbegriffe." In: Max Weber. Schriften 1894-1922. Hg. v. Dirk Kaesler. München 2002, S. 653-716.

Weber-Fas, Rudolf: Das kleine Staatslexikon. Stuttgart 2000.

Wengeler, Martin: „Gleichgewicht im Kalten Krieg. Leitvokabeln der Außenpolitik." In: Politische Leitvokabeln in der Adenauer-Ära. $\mathrm{Hg}$. v. Karin Böke/Frank Liedtke/Martin Wengeler. Berlin/New York 1996a, S. 279-323.

Wengeler, Martin: „Die Deutschen Fragen. Leitvokabeln der Deutschlandpolitik." In: Politische Leitvokabeln in der Adenauer- ̈̈ra. Hg. v. Karin Böke/Frank Liedtke/Martin Wengeler. Berlin/New York 1996b, S. 325-377.

Wengeler, Martin: „Vom Jedermann-Programm bis zur Vollbeschäftigung. Wirtschaftspolitische Leitvokabeln." In: Politische Leitvokabeln in der Adenauer-Ära. Hg. v. Karin Böke/Frank Liedtke/Martin Wengeler. Berlin/New York 1996c. S. 379-434.

Wittgenstein, Ludwig: Philosophische Untersuchungen. Frankfurt/Main 1977.

Zimmermann, Rüdiger: „Gewalt in der Sprache und durch Sprache." In: Wörter in der Politik. Analysen zur Lexemverwendung in der politischen Kommunikation. Hg. v. Hajo Diekmannshenke/Josef Klein. Opladen 1996, S. 103-121. 


\subsection{Quellen im Internet}

Arbeitsgemeinschaft „Sprache in der Politik“ e.V. $<$ www.Sprache-in-der-Politik.de> (13.09.2004)

BÜNDNIS 90/DIE GRÜNEN. <http://www.gruenenrw.de/fileadmin/user_upload/files/Allgemein/Grundsatzprogram m-BV-2002.pdf > (26.08.2004)

BÜNDNIS 90/DIE GRÜNEN/GAL Münster. <http://www.gruenemuenster.de/modules.php? op $=$ modload $\&$ name $=$ Downloads $\&$ file $=i$ ndex\&req=getit\&lid=31> (26.08.2004)

Feministische Partei DIE FRAUEN. $<$ http://www.feministischepartei.de/progra-d.htm> (03.09.2004)

PDS. <http://sozialisten.de/partei/grundsatzdokumente/programm/ programm2003.pdf $>$ (15.09.2004)

Rau, Johannes: „Wird alles gut? Für einen Fortschritt nach menschlichem Maß." Berliner Rede vom 18.05.2001. $<$ http://www.bundespraesident.de/top/dokumente/Rede/ix_41073. htm> (14.09.2004) 